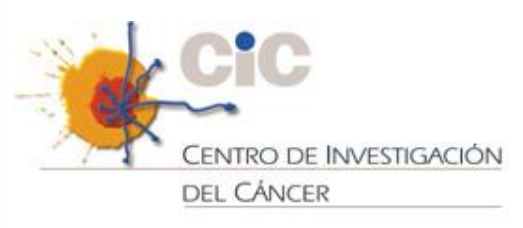

CENTRO DE INVESTIGACIÓN DEL CÁNCER INSTITUTO DE BIOLOGÍA MOLECULAR Y CELULAR DEL CÁNCER

\title{
The Pathogenesis of Ewing Sarcoma Implications of Mesenchymal Stem Cells and New Therapeutic Strategies
}

Tesis Doctoral

Ana Teresa Monteiro Amaral

Salamanca, España

2014 
D. Enrique de Álava Casado, Doctor en Medicina y Cirugía, Jefe de Servicio de Anatomía Patológica, Hospital Universitario Virgen del Rocío, e Investigador Responsable en el Instituto de Biomedicina de Sevilla,

\section{CERTIFICA:}

Que la memoria titulada "The Pathogenesis of Ewing Sarcoma. Implications of Mesenchymal Stem Cells and New Therapeutic Strategies" presentada por la licenciada en Farmacia Ana Teresa Monteiro Amaral ha sido realizada bajo su dirección en el Instituto de Biología Molecular y Celular del Cáncer y reúne, a su juicio, originalidad y contenidos suficientes para que sea presentada ante el tribunal correspondiente y optar al grado de doctor por la Universidad de Salamanca.

Y para que así conste a efectos legales, expide el presente certificado en Salamanca, de Julio de 2014.

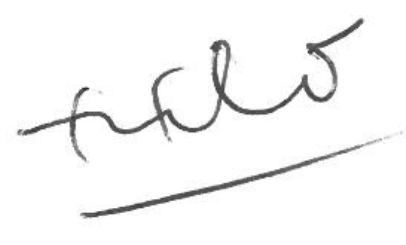

Fdo. Dr. Enrique de Álava Casado 
D. José Luís Ordoñez García, Doctor en Biología e Investigador Posdoctoral en el Instituto de Biomedicina de Sevilla,

\section{CERTIFICA:}

Que la memoria titulada "The Pathogenesis of Ewing Sarcoma. Implications of Mesenchymal Stem Cells and New Therapeutic Strategies" presentada por la licenciada en Farmacia Ana Teresa Monteiro Amaral ha sido realizada bajo su dirección en el Instituto de Biología Molecular y Celular del Cáncer y reúne, a su juicio, originalidad y contenidos suficientes para que sea presentada ante el tribunal correspondiente y optar al grado de doctor por la Universidad de Salamanca.

Y para que así conste a efectos legales, expide el presente certificado en Salamanca, de Julio de 2014.

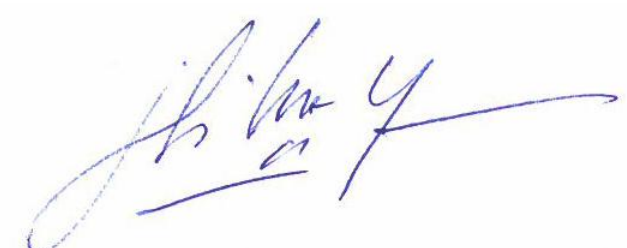

Fdo. Dr.José Luís Ordoñez García 
D. Alberto Orfão de Matos, Doctor en Medicina y Cirugía, Catedrático de Medicina de la Universidad de Salamanca, e Investigador Principal en el Instituto de Biología Molecular y Celular del Cáncer de Salamanca,

\section{CERTIFICA:}

Que la memoria titulada "The Pathogenesis of Ewing Sarcoma. Implications of Mesenchymal Stem Cells and New Therapeutic Strategies" presentada por la licenciada en Farmacia Ana Teresa Monteiro Amaral ha sido realizada bajo su tutoría en el Instituto de Biología Molecular y Celular del Cáncer y reúne, a su juicio, originalidad y contenidos suficientes para que sea presentada ante el tribunal correspondiente y optar al grado de doctor por la Universidad de Salamanca.

Y para que así conste a efectos legales, expide el presente certificado en Salamanca, de Julio de 2014.

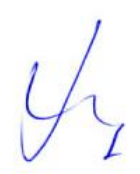

Fdo. Dr.Alberto Orfão de Matos 
Este proyecto de tesis ha sido financiado por:

- La Fundação para a Ciência e Tecnologia, Governo de Portugal, con el Proyecto: "The pathogenesis of Ewing Sarcoma: implications for novel treatment strategies" (SFRH/BD/69318/2010).

- La Red de Excelencia de la Comunidad Europea EUROBONET, con el Proyecto Europeo "Molecular Pathology of Bone Tumors" (FP6-2004Lifescihealth-5, 018814).

- European Clinical trials in Rare Sarcomas within an integrated translational trial network (EUROSARC) FP7-HEALTH-2011-two-stage (Project ID 278742 Comisión Europea)

- El Ministerio de Ciencia e Innovación, a través del Instituto de Salud Carlos III (RD12/0036/0017, RD06/0020/0059, PI1203102, Pl1100018, PI081828, PI052524)

-La Fundación Asociación Española Contra el Cáncer (AECC), con el proyecto "Búsqueda, validación y traslación clínica de nuevas dianas terapéuticas a partir de estudios de genómica y epigenómica integrativa en sarcoma de Ewing". (GCB13131578DE Á) 


\section{Table of Contents}

\section{Glossary of Abbreviations}

\section{List of Figures}

\section{List of Tables}

Introduction. 1

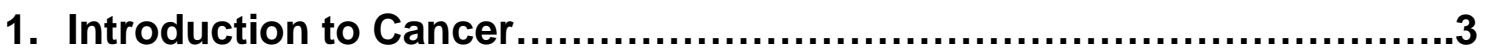

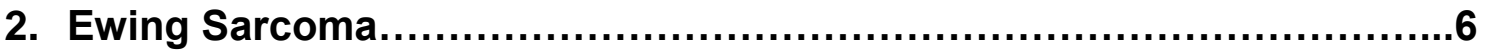

2.1. From Clinical Features to Prognosis................................... 6

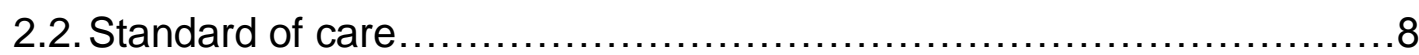

3. The dilemma of ES histogenesis.............................................10

3.1. The Mesenchymal Stem Cell: Definition of the term......................10

3.1.1. Characterization and properties of MSC .......................11

3.2. The possible implication of MSC in ES initiation: facts and

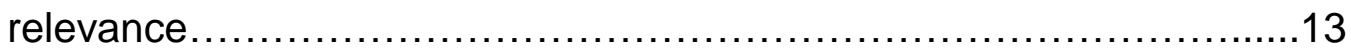

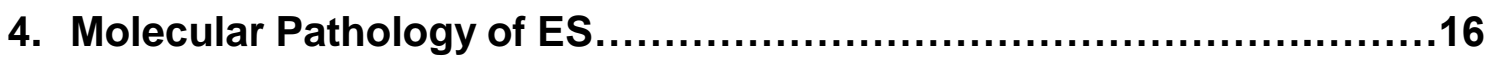

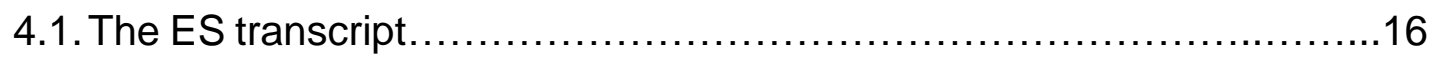

4.1.1Targeting the EWSR1-FLI1 fusions..............................17

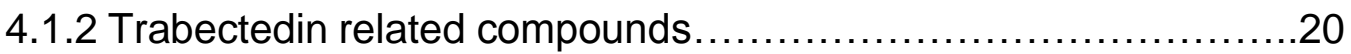

4.2. CD99: an over expressed antigen and a target option in ES .............23

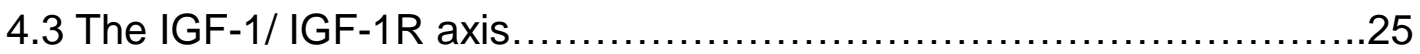

4.3.1 Clinical relevance and implications...............................25

4.3.2 Anti-IGF-1R Monoclonal Antibodies................................27

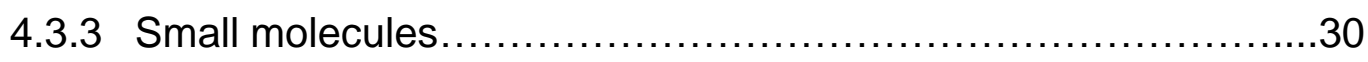

4.4 PARP1 and PARPi: General Review and facts in ES....................31 
5. Overview on the DNA Damage and Repair pathway.

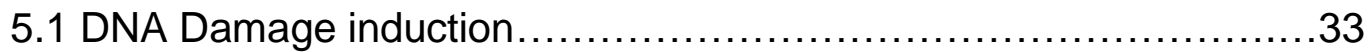

5.2 Activation of DNA damage repair pathways (DDR) .....................35

5.3 Trabectedin: crosstalk with NER and further DDR activation..........38

5.4 Trabectedin related compounds and DDR activation...................39

5.5 Olaparib and DDR activation.......................................40

Hypothesis and Objectives.......................................................43

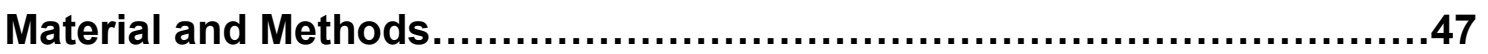

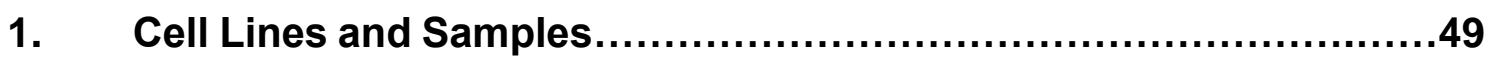

1.1 Tumor cell lines characterization and long-term culture...............49

1.2 Mesenchymal Stem Cell isolation, expansion and long-term culture .................................................................

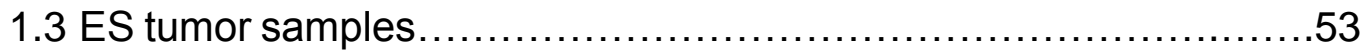

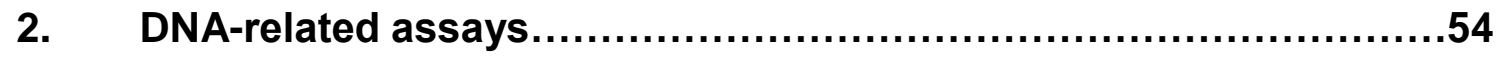

2.1 DNA extraction.............................................................

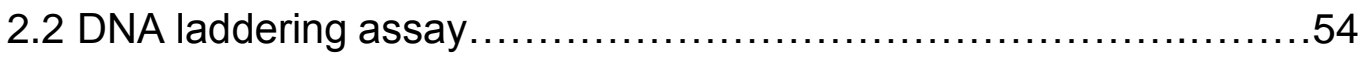

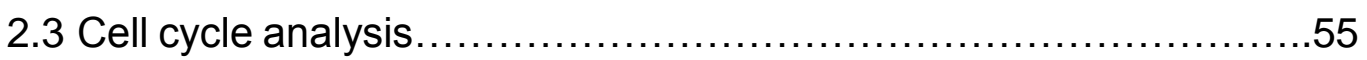

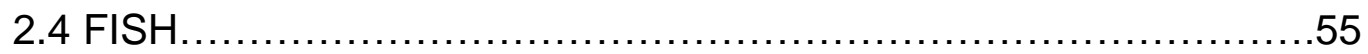

2.4.1 EWSR1 break-apart probes and EWS-FLI1 fusion

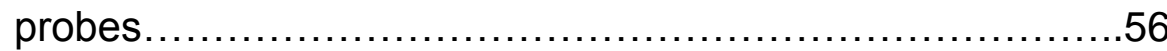

2.4.2 PARP1 probes......................................................

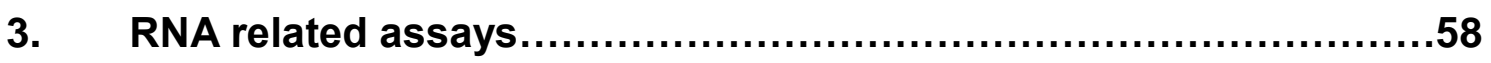

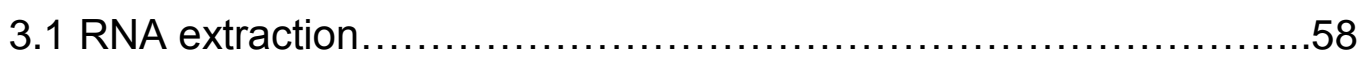

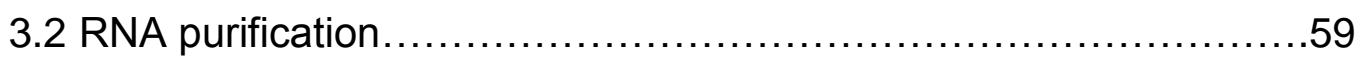

3.3 RNA quality evaluation............................................ 60

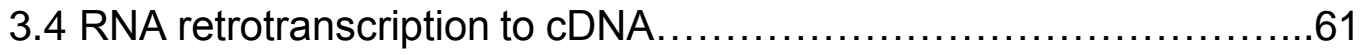

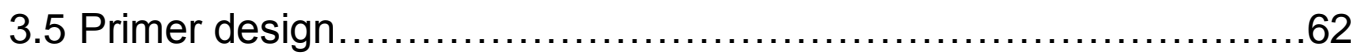

3.6 Quantitative RT-PCR ............................................... 62

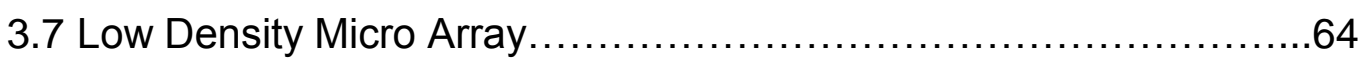

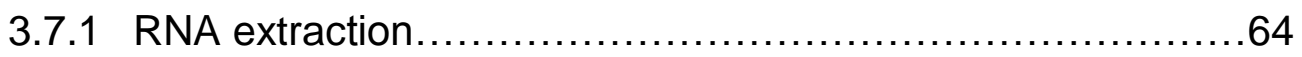

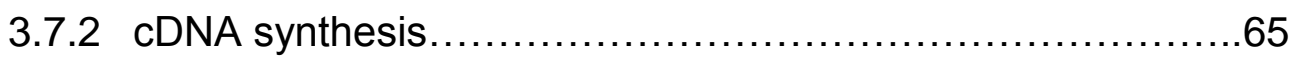


3.7.3 Quantitative RT-PCR for LDMA..........................65

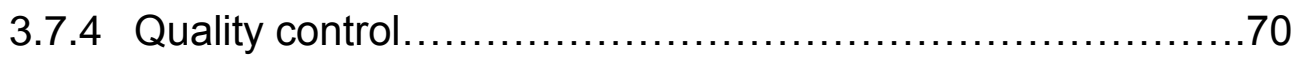

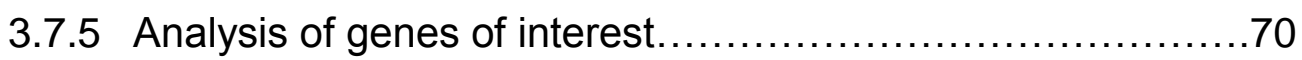

4. Protein related assays ..................................................71

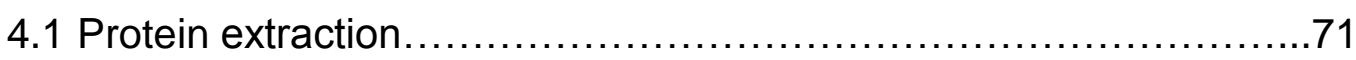

4.2 Protein quantification: the Bradford method.......................71

4.3 Electrophoresis and Transference.............................72

4.4 Blocking, Incubation with primary and secondary antibodies and Western Blotting development....................................

4.5 Flow Cytometry ..............................................

4.5.1 Caspase-3 detection assay...........................75

4.5.2 Multiparameter Flow Cytometry .........................75

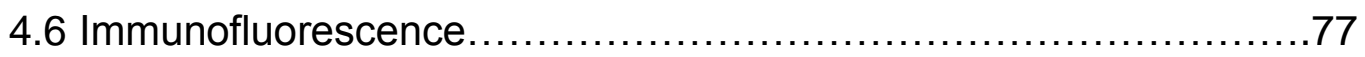

5. Invasion and Migration Assays..................................... 79

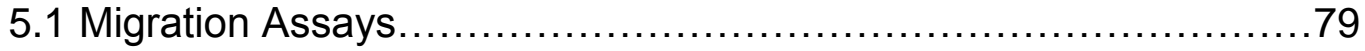

5.2 Invasion Assays.................................................

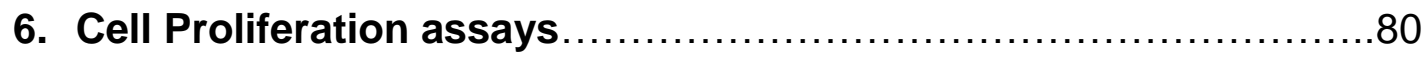

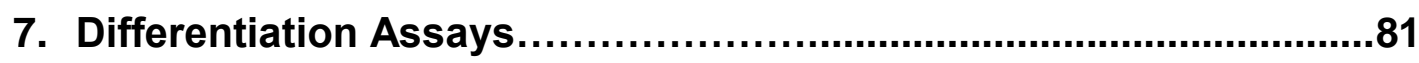

7.1 Osteogenic Differentiation.................................... 81

7.2 Adipogenic Differentiation......................................... 82

7.3 Chondrogenic Differentiation ..................................83

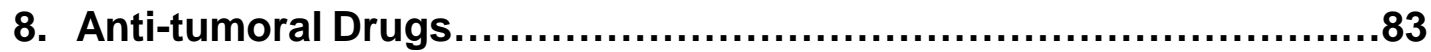

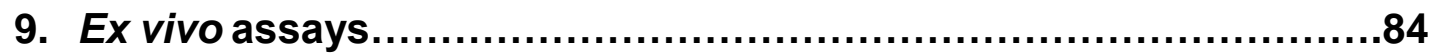

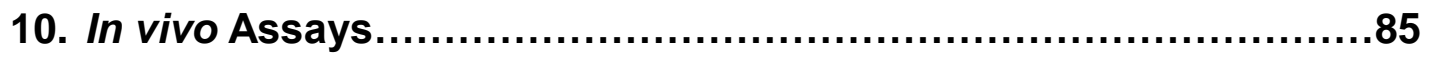

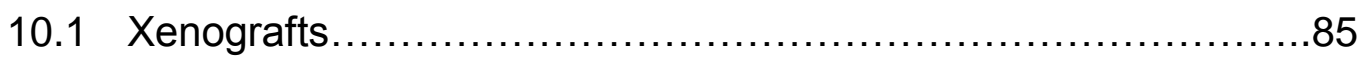

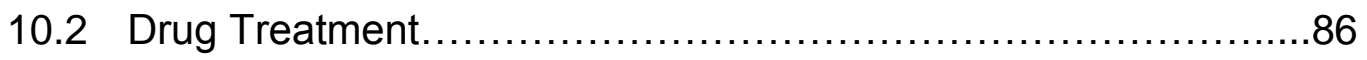

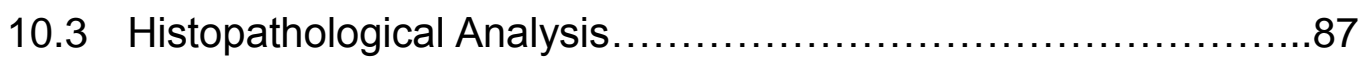

10.4 Immunohistochemistry Analysis.............................. 88

11. Statistical Analysis and bio-software...............................88

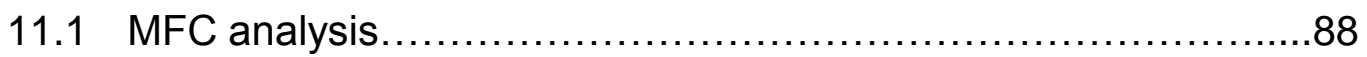

11.2 Hierarchical Clustering Analysis................................ 89

11.3 Correlation of IC50 and cell lines and determination of Combination

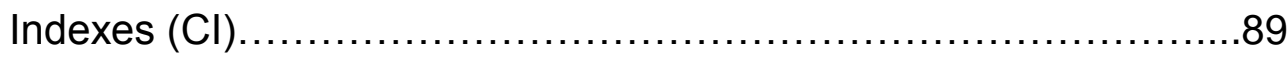


Results.

1. Immunophenotype and its implication in ES biology and treatment....95

1.1. Characterization of MSC .............................................. 97

1.2.MSC derived from ES Patients lack EWSR1 gene

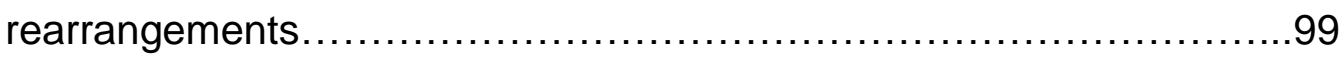

1.3.MSC derived from ES Patients have a low expression of ES

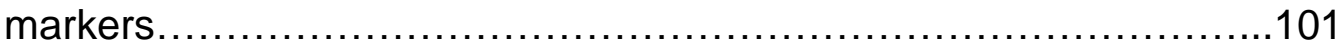

1.4. The plasma membrane expression pattern of ES cell lines is globally different from that of MSC

1.5. MSC derived from ES patients presented lower levels of CD99 expression than that of ES cells 106

1.6. CD99 is heterogeneously expressed in MSC from different tissue sources.

1.7. CD99 engagement does not elicit impairment of MSC survival. 110

2. Comparative studies on Marine compounds Trabectedin, Zalypsis and Lurbinectedin activity in Ewing Sarcoma 113

2.1 Trabectedin inhibits proliferation in all ES cells at the sub-nanomolar Range. 115

2.2 Trabectedin derived compounds induced cytotoxicity in ES cells 116

2.3 Zalypsis and Lurbinectedin are unable to overcome resistance to Trabectedin. 119

2.4 Trabectedin, Zalypsis and Lurbinectedin induce DSB in ES cells.

2.5 Trabectedin, Zalypsis and Lurbinectedin, induce activation of DDR pathways.

3. Trabectedin efficacy in ES is greatly increased by combination with antiIGF1 signaling agents. 127 
3.1 Trabectedin disrupts EWSR1-FLI1 binding to some DNA targets but increases recruitment to IGF-1R promoter either in in vitro or ex vivo models.

3.2 Anti-tumor activity of the combination between Trabectedin and the anti IGF-1R HAb AVE1642.

3.3 Efficacy of the dual inhibitor anti-IGF-1R/IR Linsitinib in combination with Trabectedin. 134

4. PARPi Olaparib enhances sensitivity of Ewing Sarcoma Cells to Trabectedin

4.1 ES cells are especially sensitive to PARPi Olaparib, which induces G2/M accumulation, independently of the $1 q$ status.

4.2 Combination of Olaparib and Trabectedin results is highly synergistic in ES cell lines. .148

4.3 The combination of Olaparib and Trabectedin enhances DNA damage induction

4.4 Olaparib and/or Trabectedin provoked the deregulation of DDR machinery..... 154

4.5 Drug combination dramatically reduced tumor growth in an in vivo model. 158

4.6 The drug combination increased DNA damage induction and DDR activation also in the in vivo model. 162

Discussion 167

1. The role of the MSC axis in ES biology. Targeting CD99 in MSC. 169

2. The establishment of innovative therapies in ES treatment: outcome from the in vitro and in vivo studies. .172

2.1. The relevance of Trabectedin, Zalypsis y Lurbinectedin in ES therapy: outcome from in vitro studies.

2.2. The combination of the dual inhibitor Linsitinib with Trabectedin........174

2.3. PARPi in ES. The combination of Trabectedin and Olaparib. 178 
3. Final Outcome and future perspectives...................................182

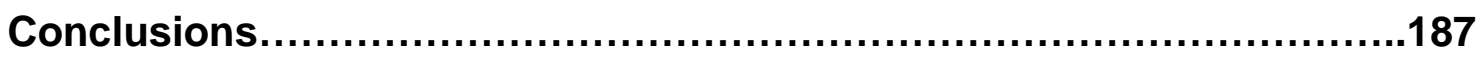

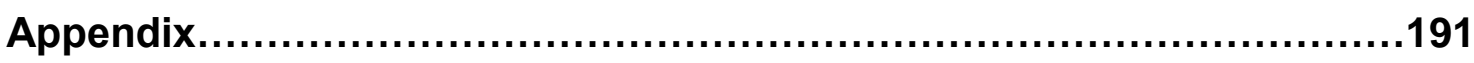

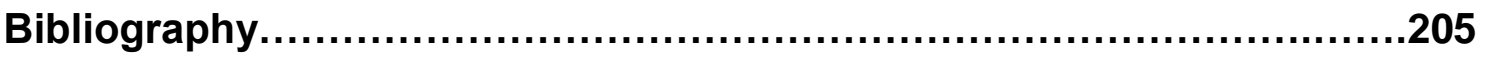




\section{Glossary of Abbreviations}

A

AD: Adipose derived

AM: Amnios derived

Ag: Antigen

ARA-C: Cytarabine

ATM: Ataxia Talangiectasia Mutated

ATR: Ataxia Talangiectasia and RAD3 related

APS: Ammonium-Persulphate

\section{B}

BER: Base Excision Repair

BM: Bone Marrow

BRCA1: Breast Cancer Associated 1 BRCA2: Breast Cancer Associated 1

BA: Break Apart

BSA: Bovine Serum Albumin

C

Cor: Chorion

CT: Clinical Trial

CAV1: Caveolin-1

CD99: MIC2

CD105: Endoglin

CD73: Ecto-5'-nuceotidase

CD90: Cluster of differentiation 90, Thy-1
CD45: Cluster of differentiation 45

CD117: C-KIT, tyrosine kinase receptor

CD271: Low affinity nerve growth factor receptor

$\mathrm{Cl}$ : Combination Indexes

D

DNA: Deoxyribonucleic Acid

DDR: DNA damage repair

DXR: Doxorubicin

DP: Dental Pulp

DSB: Double Strand breaks

DNA-PKcs: DNA-dependent protein kinase catalytic subunit

DEPC: Diethylpyrocarbonate

DMSO: dimetil sulfóxido

DMEM: Dulbeco's modifiedminimum essential medium

DAPI: 4',6',DiAmidino-2-Phenil Indol

E

ES: Ewing Sarcoma

EDTA: ehtylenedaimine-tetracetic acid 
FBS: Fetal Bovine Serum

FISH: Fluorescence in situ $\mathbf{K}$

Hybridization

kDa: Kilodalton

kb: Kilo base

G

GDC: Genomic DNA Control

H

LDMA: Low Density Micro Arrays

HR: Homologous Recombination

HLA-DR: Human Leukocyte AntigenDR

hTERT: human Telomerase

HSP: Heat Shock Protein

HAb: Humanized Antibody

HNSCC: Head and Neck Squamous Cancer

HUSAL: Hospital Universitário de Salamanca

$\mathrm{H}$ : hour

H2AX: Histone 2AX

I

IF: Immunofluorescence

IHC: Immunohistochemistry

IGF1R: Insulin Growth Factor Receptor 1

IR: Insulin Receptor

M

MSC: Mesenchymal Stem Cell

MMR: Mismatch Repair

MSC-EP: MSC derived from ES patients

MSC-HD: MSC derived from Healthy Donors

Mg: milligram

mM: mili Molar

ml: milliliter

M: Molar

MFC: $\quad$ Multiparameter $\quad$ Flow Cytometry

MAb: Monoclonal Antibodies

MMR: Mismatch Repair

mRNA: Messenger RNA

Ms: Mouse

MFI: Median Fluorescence Intensity

ISCT: International Society for Cellular Therapy

IGFBPs: IGF Binding Proteins

ICAM: Intercellular Cell Adhesion Molecule 
rpm: revolutions per minute

NHEJR: Non-Homologous End Joining Repair

RT-PCR: Reverse transcriptase PCR

NER: Nucleotide Excision Repair

NCT: National Clinical Trial

NSC: Neural Stem Cells

NCSC: Neural Crest Stem Cells

nM: nanoMolar

RPA: Replication protein A

S

SBT: Sarcomas Bearing Translocations

SSB: Single Strand Breaks

SDS: Sodium dodecylsulfate

O

SDS-PAGE: SDS- Polyacrylamide

OGG1: 8-oxoguanine-glycosylase Gel

$\mathbf{T}$

TK: Tyrosine-Kinase

TAE: Tris-Acetate-EDTA

P/S: Penicillin/Streptomycin

PCR: Polymerase Chain Reaction

PARP1: poli-(ADP-ribose) polymerase-1

PARPi: PARP inhibitors

PPTP: Pediatric Preclinical Testing TBS-T: Tris-Buffer Saline with Tween-20

U

UV: Ultraviolet Radiation Program

PPC: Positive PCR Control

$\mathbf{R}$

Molecule

RHA: RNA Helicase-A

RPMI: Roswell Park Memorial Institute

WB: Western Blotting

RT: Room Temperature

WT: Wild type

RNA: Ribonucleic Acid

RTC: Reverse Transcription Control

$\mathrm{Rb}$ : Rabbit

$\mathbf{X}$

XP: Xeroderma Pigmentosum 


\section{List of Figures}

Figure 1. Schematic representation of the hallmarks of cancer .................4

Figure 2. Graphic representation of the complex group of soft-tissue sarcomas, according to the chromosomal translocation...................................

Figure 3. Innovative pre-clinical/clinical therapeutic approaches in ES treatment.

Figure 4. MSC can be isolated and expanded from several tissues and, under the appropriate stimuli, can differentiate into cell types such as osteoblasts, adipocytes, chondrocytes and others ...................................... 13

Figure 5. Chemical Structures of Trabectedin and Related compounds........21

Figure 6. The IGF-1/ IGF-1R downstream pathway..........................26

Figure 7. Simplified schematic view of DNA damage induction, with $\mathrm{H} 2 \mathrm{AX}$ phosphorylation, presence of intra-nuclear foci of $\mathrm{\gamma H} 2 \mathrm{AX}$ and further activation of sensor/mediator/effector molecules with the final goal of DNA repair by

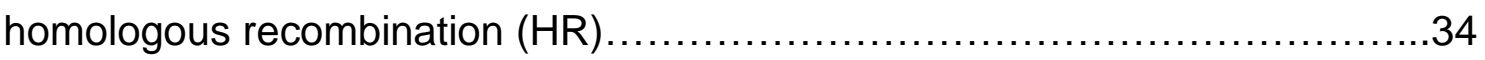

Figure 8. Graphic Representation of DNA damaging agents and the type of lesion induced as well as the major repair pathways activated by each group of drugs.

Figure 9. Schematic view of BER, HR, NER and NHEJ, DNA repair pathways and molecules involved.

Figure 10. Confirmation on $1 \%$ agarose gels of DNA probe labeling for RPPE964L17

Figure 11. Ribosomal RNA identification

Figure 12. Evaluation of the specificity of the set of primers, in a 1\% agarose-gel, to be used in q-RT-PCR.

Figure 13. Example of melting peak with a single curve, as obtained by the iQ5 BIORAD software. .64

Figure 14. Distinct combinations of agents to fix and permeabilize cells.......77

Figure 15. Invasion Assays schematic system ................................. 80

Figure 16. Schematic structure of the in vivo assay........................... 87

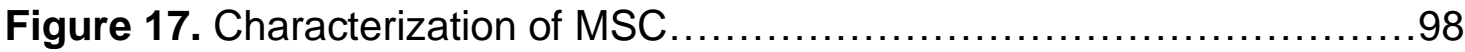

Figure 18. Immunophenotypic characterization of MSC ........................99 
Figure 19. MSC-P lack the presence of the EWSR1-FLI1 chromosomal

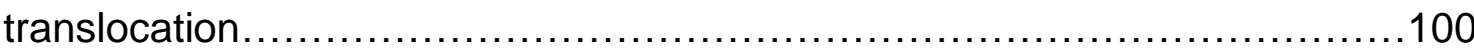

Figure 20. Immunophenotype of MSC-P versus ES cells.....................101

Figure 21. Comparative study between MSC, ES cell lines and ES samples....................................................................... 103

Figure 22. IHC studies in frozen tissue from ES samples.....................104

Figure 23. Expression of CD105, CD34, CD90 and CD45 ...................105

Figure 24. CD99 expression in MSC and ES cell lines.......................106

Figure 25. Cytofluorimetric analysis of CD99 expression on RD-ES cell line and BM-MSC.

Figure 26. Percentage of living, apoptotic and necrotic cells observed in cells treated with anti-CD99 MAb for 15 min or $4 \mathrm{hrs}$

Figure 27. Caspases -3 and -7 activity after 24 hour of drug incubation in two ES cell lines. 116

Figure 28. Migration Assays on the TC71 cell line after 72 hour of drug incubation. 118

Figure 29. Cell cycle profiling on the $A 4573$ cell line after 24 hours of drug incubation. 118

Figure 30. The resistant cell line is also resistant to Zalypsis and Lurbinectedin. 119

Figure 31. DNA Damage induction induced by treatment with Trabectedin/Zalypsis/Lurbinectedin 121

Figure 32. Induction of DSB after drug washout. DSB were persistent 122

Figure 33. Western Blotting analysis of DDR protein expression on two ES cell lines after treatment. 125

Figure 34. Trabectedin caused a dysregulation in the binding of EWS-FLI1 chimera to specific promoters 129

Figure 35. Trabectedin caused a dysregulation in the binding of EWS-FLI1 chimera to specific promoters

Figure 36. Increased recruitment of EWS-FLI1 on IGF-1R promoter in TC-71 and 6647 EWS cells treated for $1 \mathrm{~h}$ with Trabectedin or DX 131

Figure 37. Time course of FLI1 association with the IGF-1R promoter in TC-71 and 6647 xenografts treated with trabectedin $(0.15 \mathrm{mg} / \mathrm{kg})$. 132

Figure 38. Efficacy of the combination of Trabectedin with the 
anti-IGF-1R HAb AVE1642 against TC-71 xenografts

Figure 39. Representative immunohistochemical evaluation of

Ki-67, apoptotic rate by TUNEL assay and neural differentiation markers

Figure 40. Efficacy of the dual inhibitor anti-IGF-1R/IR OSI-906 in combination with Trabectedin: Protein expression

Figure 41. Efficacy of the dual inhibitor anti-IGF-1R/IR OSI-906 in

combination with Trabectedin: Cell cycle profiling and apoptosis induction.

Figure 42. DNA damage induction after cell exposure to Trabectedin

and/or OSI-906.

Figure 43. Evaluation of Caspase 3 and PARP cleavage by western

blotting after $24 \mathrm{~h}$ exposure to drugs.

Figure 44. DNA damage repair pathways after treatment of

TC-71 cells with Trabectedin and/or Osi-906.

Figure 45. PARP1 expression in ES cell lines.

Figure 46. ES cell lines Sensitivity to PARPi: Olaparib,

Veliparib and Iniparib.

Figure 47. ES cell sensitivity induces $\mathrm{G} 2 / \mathrm{M}$ cell cycle accumulation and is not correlated to $1 \mathrm{qG}$.

Figure 48. The combination of Trabectedin and Olaparib: effects

on caspases activity and cell cycle profiling

Figure 49. DNA damage induction studies: Short-term damage induction.

Figure 50. DNA damage induction studies: Long-term damage induction.......152

Figure 51. DNA damage induction studies: Fragmentation Studies.

Figure 52. Schematic representation of gene interactions in cells

treated with Trabectedin, Olaparib or combination of

Olaparib and Trabectedin.

Figure 53. In vivo model using the TC71 cell line.

Figure 54. In vivo model using the TC71 cell line:

Hystopathological analysis. 160

Figure 55. In vivo model using the TC71 cell line: Toxicity analysis. 161

Figure 56. Immunoflorescence and Immunohistochemistry study

of tumor xenografts: Apoptosis,proliferation

Figure 57. Immunofluorescence and Immunohistochemistry

study of tumor xenografts: DNA damage Induction and repair. .164 
Figure 58. Hypothetic malignant process: from hMSC to pre-malignant stages and fully malignant ES cells.

Figure 59. Complex pathways of action of different drugs on ES cells........185

Figure 60.S Anti-tumor activity of Trabectedin and DXR in xenografts........198

Figure 61.S Recruitment of IGFF1R promoter by EWS-Fli1....................199

Figure 62.S Proliferation Inhibition after treatment with Olaparib...............200

Figure 63.S Migration assays in RM82 cell line after treatment...................200 


\section{List of Tables}

Table I. Models of sarcoma development using MSC.

Table II. Frequency of chromosomal translocations in ES and

Ewing-like Sarcomas.

Table III. Relationship between drugs and DDR

Table IV. ES cell lines characterization...................................... 50

Table V. Characterization of BM-MSC from ES Patients.......................51

Table VI. Characterization of MSC derived from healthy donors................52

Table VII. Primers used for RT-PCR and q-RT-PCR ...........................63

Table VIII. Description of the genes included in PCR array .....................66

Table IX. Standards/sample for protein quantification by the Bradford

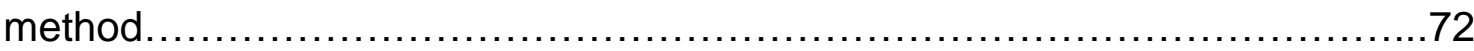

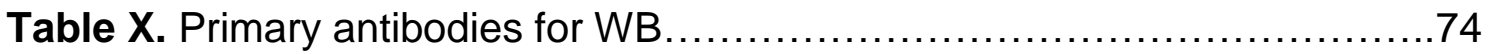

Table XI. Panel of antibodies and Fluorescence used for MFC ..................76

Table XII. Primary Antibodies used for IF ........................................78

Table XIII. Description of the anti-tumoral agents................................. 84

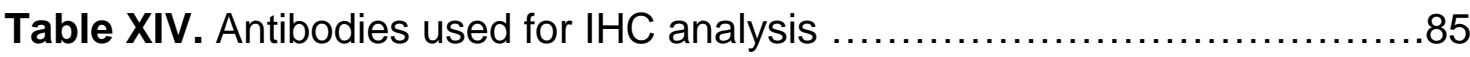

Table XV. Antibodies used for histochemistry analysis in xenografts

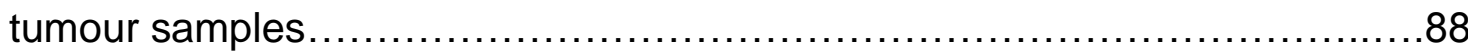

Table XVI. CD99 intensity levels on MSC-P as assessed by MFC .............108

Table XVII. CD99 expression in MSC derived from healthy tissues............106

Table XVIII. Proliferation IC 50 indexes after 72 hour of drug

incubation with Trabectedin/Zalypsis/Lurbinectedin.

Table XIX. Apoptosis /Necrosis induction after treatment with 500pM of Trabectedin/Zalypsis/Lurbinectedin ............................................117

Table XX. IC 50 Indexes of proliferation on the TC-ET 12nM compared to TC71 to Zalypsis and Lurbinectedin.

Table XXI. Description of the up-regulated genes after 18 hour of treatment on the TC71 cell line.

Table XXII. Sensitivity of ES cell lines to the dual IGF1R/IR inhibitor OSI-906.

Table XXIII. Efficacy of combined treatments of Trabectedin with 
OSI-906 in ES cell lines 136

Table XXIV Combination of Olaparib and Trabectedin in ES cell lines.

Table XXV. Expression changes in DNA damage repair genes in ES cells from the TC71 cell line.

Table XXVI.S Fold Change in the TC71 cell line after treatment with 500pM pf Trabectedin, Zalypsis and Lurbinectedin

Table XXVII.S Fold Change in the TC71 cell line after treatment with 200pM pf Trabectedin, Zalypsis and Lurbinectedin 195

Table XXVIII.S. Fold Change from the LDMA after treatment with Trabectedin and/or Olaparib. 201 
For every child struggling with cancer

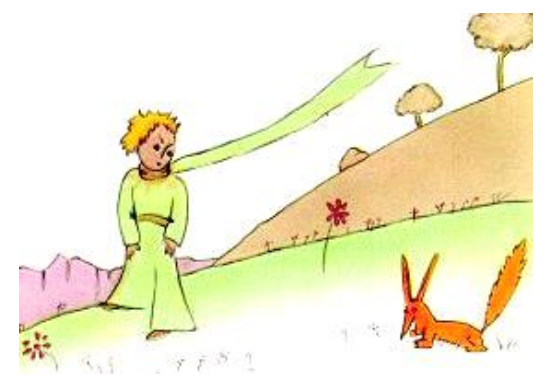




\section{Introduction}


The Pathogenesis of Ewing Sarcoma / Introduction 


\section{Introduction to Cancer}

The World Health Organization (WHO) reported that cancer accounts for 7.6 million deaths per year worldwide (data from 2008) hence representing a leading cause of mortality (http://www.who.int/cancer/en/index.html). This group of diseases has been intensively studied and, according to Hanahan D. and Weinberg R., who brilliantly summarized and updated the features that allow the raise and development of tumors into six main hallmarks, which are:

i) Self-sufficiency based in growth signals;

ii) Capacity to overcome inhibitory signals;

iii) Capacity to escape death signals and apoptosis;

iv) Constant ability to replicate;

v) Angiogenesis;

vi) Capacity to Invade and Metastasize.

Recently, given the enormous contribution on cancer research at the basic to pre-clinical and clinical levels, new hallmarks have emerged, mostly those related to tumor microenvironment and the ability to escape the immune system together with the deregulation of cellular energetic mechanisms, as reflected in Figure 1.[1, 2] Two main enabling characteristics were added to this group as they facilitate the acquirement of the other hallmarks: genetic instability and/or mutation and tumor promoting inflammation.[2] 


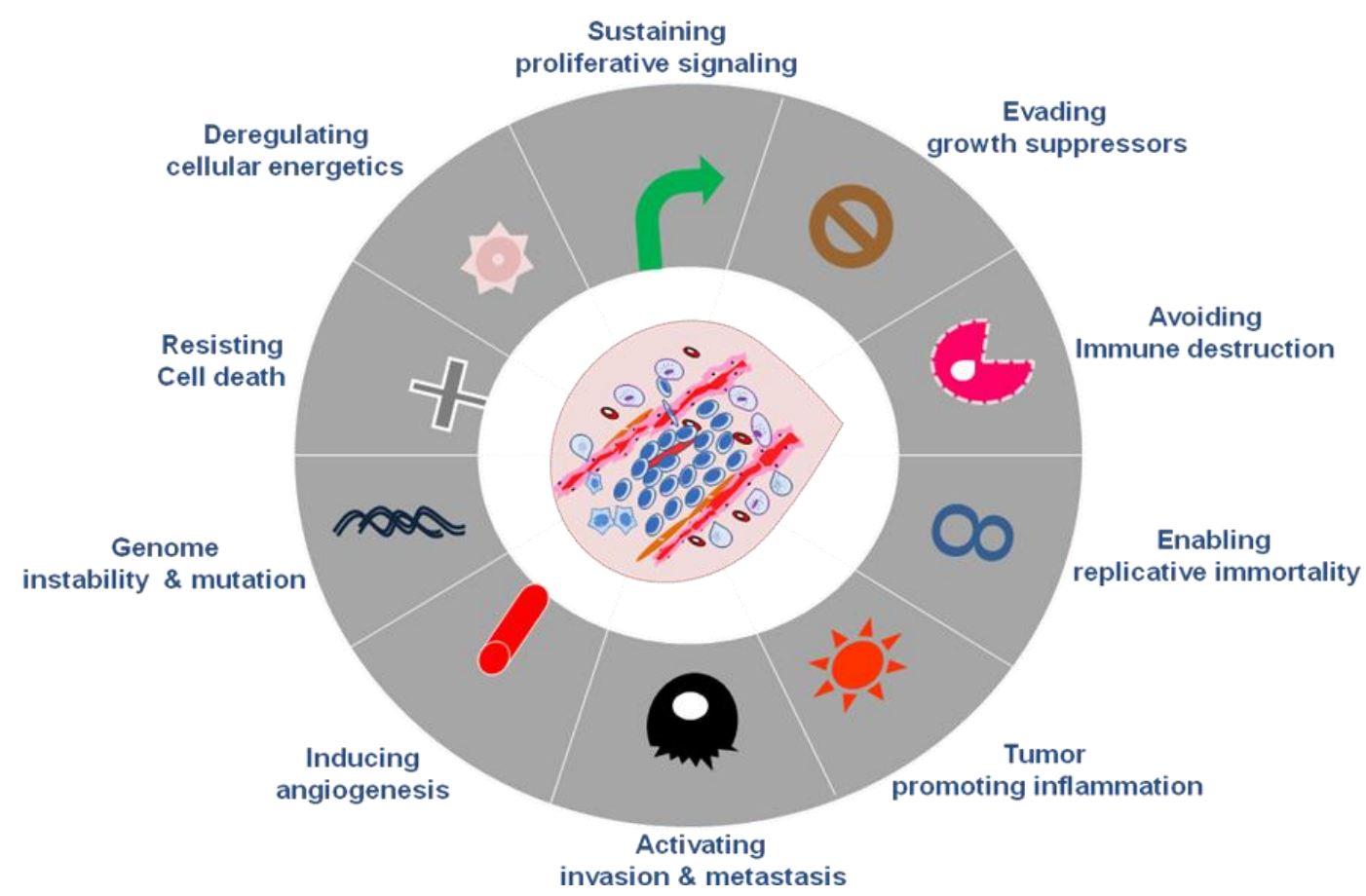

Figure 1. Schematic representation of the hallmarks of cancer (adapted from to Hanahan D. and Weinberg R., 2011, Cell).

Tumors can be divided into several groups. Broadly they can be divided into hematological or solid. Within solid tumors, there is also much complexity, and here tumors can be divided into carcinomas, sarcomas, melanomas, neural tumors, among others, depending on the cell type from which they differentiate.

Sarcomas are a rare group of solid tumors, with an incidence of less than $10 \%$ of all cancers, displaying a high degree of molecular/genetic complexity (Figure 2), as well as mesenchymal differentiation.[3] In general, they are very frequent in children and young adults and differ from other tumors in terms of molecular and pathological features. From a genetic point of view there are two groups of sarcomas: i) sarcomas with complex karyotypes and ii) sarcomas with simple karyotypes, which are mostly characterized by the presence of recurrent balanced chromosomal translocations as the major genetic component of this entity.[4] The following figure reflects the different 
chromosomal translocations present within the different sarcoma types. Among the global sarcoma group, Ewing sarcoma (ES) is the second most common primary childhood/young adult bone sarcoma, after osteosarcoma.

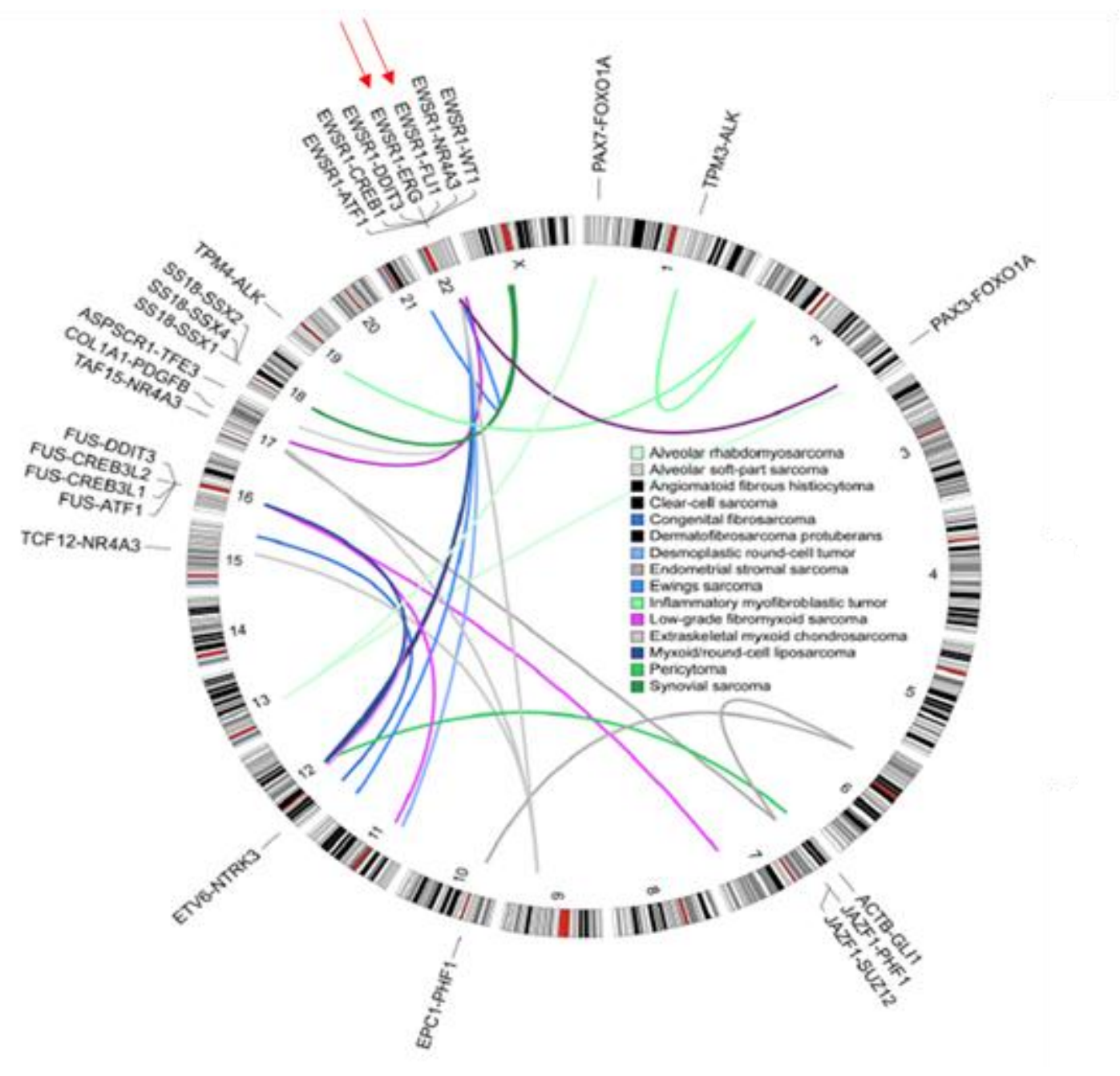

Figure 2. Graphic representation of the complex group of soft-tissue sarcomas, according to the chromosomal translocation. The outer ring localizes the genes enrolled in the translocation from chromosome 1 to chromosome $X$ and the curves represent the union of the fusion partners. Red arrows localized the most frequent balanced translocation in ES, EWSR1-FLI1, as well as the second most common translocation EWSR1-ERG (modified from Taylor B et al, Nature Reviews on Cancer, 2012). 


\section{Ewing Sarcoma:}

\subsection{From Clinical Features to Prognosis}

ES is a small round cell sarcoma, relatively uncommon, accounting for 6$8 \%$ of primary malignant bone tumors. ES shows a slight predilection for males, with the ratio of 1.4 to 1.[5] Moreover, nearly $80 \%$ of patients are younger than 20 years, and the peak age incidence is during the second decade of life. ES can occur in patients over 40 , although in this age group it is characterized by a similar spectrum of EWSR1-ETS gene fusions than in childhood.[6]

ES is molecularly characterized by the presence of recurrent balanced translocations involving, in almost all cases, the EWSR1 gene on chromosome 22 and a member of the ETS family of transcription factors. This leads to the formation of novel fusion oncogenes that are the key to pathogenesis. EWSR1ETS fusion proteins activate or repress specific sets of target genes that, together with the right timing and cellular context, give rise to the transformed phenotype of ES.

ES clinical presentation can include a severe pain, enough to wake up patients $(96 \%)$, with or without a mass in the involved area (61\%). Moreover, also includes intermittent fever (21\%), anemia, and other unspecific lab results. Pathological fractures (16\%) at diagnosis are uncommon. Radiographically, an ill-defined osteolytic lesion involving the diaphysis of a long tubular bone is the most common feature. Permeative or motheaten bone destruction often associated with "onion-skin"-like multilayered periosteal reaction is also characteristic. A large, ill-defined soft tissue mass is a frequent association. Magnetic resonance imaging studies help demonstrate the extent of the tumor in the bone and soft tissue. The most prevalent sites are, in descending order: 
i) The diaphysis or metaphyseal - diaphyseal portion of long bones (femur, tibia, humerus);

ii) Pelvis and ribs;

iii) Other bones, such as the skull, vertebra, scapula, and short tubular bones of hands and feet;

iv) Only about $10 \%$ of cases are extra-skeletal.

The prognosis in ES has improved considerably with current therapy, and approximately two thirds of patients show a remission of their disease.[7] However, outcome for patients with disseminated disease or early relapse remains dismal, and the presence of metastatic disease is the major adverse prognostic factor. $[8,9]$

Other important prognostic features include the stage, anatomic location, and the size of the tumor. Histopathological assessment of tumor necrosis after induction chemotherapy has prognostic value. Several molecular features were reported to have prognostic value, including p53 status, INK4A loss, telomerase expression, percentage of genome altered, and additional chromosomal aberrations such as gains of chromosome 1q.[10] However, clinical application of molecular prognostic factors still requires validation in prospective cooperative studies.[10]

EWSR1-ETS fusion molecular subtype was initially reported to provide prognostic information. Among localized tumors with EWSR1-FLI1 gene fusions, those with the most common type 1 EWSR1 exon 7/FLI1 exon 6 fusions had a better prognosis than cases with other fusion types. However, the use of more intensive therapeutic regimens appears to have eliminated these differences.[11, 12] 


\subsection{Standard of care}

Local therapy supplemented with chemo/radiotherapy is the current standard of care for a large number of solid malignant tumors including ES. Accordingly, this type of treatment does not specifically target the pathogenetic mechanisms of ES cells, and therefore drug-related resistance frequently emerges (Figure 3).

First line therapy consists of neoadjuvant therapy, usually combining 4 to 6 chemotherapeutic agents among vincristine (V), doxorubicin (DXR) (D), etoposide (E), cyclophosphamide (C), ifosfamide (I) and/or dactinomycin (D) also named actinomycin-D (A), followed by surgery and/or radiotherapy whenever appropriate.[8] The most widely combination used in Europe is VIDE/VAI whereas in the US is VDC/IE.[13] These multimodality treatments are able to increase overall survival up to $75 \%$ in localized disease. Nevertheless, in cases of multifocal disease, $5+$ year overall survival is below $20 \%$.[13, 14$]$

Under the Euro-EWING99 Clinical Trial (CT) (NCT00020566) patients presenting primary or disseminated multifocal ES, followed several cycles of multimodal therapeutic combinations of VIDE, VAI, radiotherapy, surgery and high-dose chemotherapy (busulfan - melphalan) with autologous stem celltransplantation. Outcome from this trial revealed that the best approach resulted from the combination of local treatment with surgery and/or radiotherapy plus multimodal intensive therapeutic strategies.[8, 14] Here, prospective studies from this CT determined a lack of prognostic benefit related to the molecular subtype of the fusion transcript EWSR1-FLI1, in contrast to previous studies including a shorter number of patients.[11, 15] 


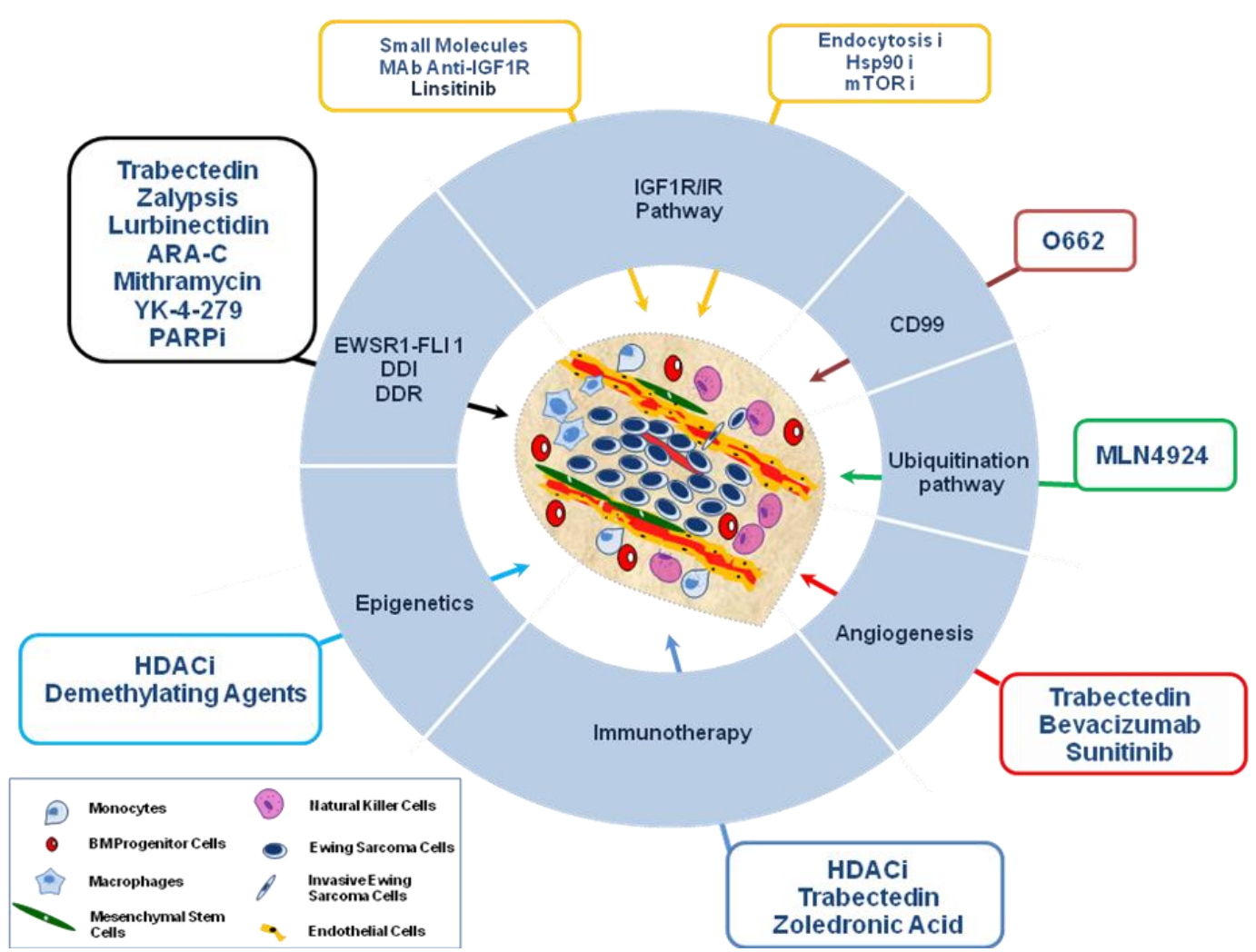

Figure 3. Innovative pre-clinical/clinical therapeutic approaches in ES treatment (Obtained from Amaral AT \& Ordoñez JL et al. 2014)

Under the ongoing Phase III CT, EWING 2008 (NCT00987636), patients with localized and disseminated ES were randomized into three broad groups according to standard risk: 1) patients with localized disease with tumor volume $<200 \mathrm{ml}$ are being treated with zoledronic acid and/ or the retinoid derivative fenretinide in addition to induction chemotherapy (VIDE); 2) patients with localized/metastasis disease with tumor volume $>200 \mathrm{ml}$ are being treated with high dose chemotherapy (busulfan-melphalan) and autologous stem cell transplantation; 3) patients with primary disseminated disease, are being treated with 8 cycles of standard adjuvant chemotherapy (VIDE/VAI) and 
followed by high dose chemotherapy (treosulfan - melphalan) plus autologous stem cell transplantation.

Regarding relapsed and metastatic disease, in which patients experience a much worst prognosis, there are several non-randomized ongoing CT at the moment. These studies include treatment with Trabectedin and its related compound Zalypsis (NCT01222767); Src family tyrosine kynase inhibitor Dasatinib (NCT01643278); or proteasome inhibitor bortezomib (NCT00027716) among others, in monotherapy, as well as other combinatory regimens.

\section{The dilemma of ES histogenesis}

The origin of ES has long been the focus of research over the years. In fact, initially, neural stem cells (NSC) were pointed as the most probable cell of origin. However, since mesenchymal stem cells (MSC) have been characterized and studied in depth, these cells redirected the theme of ES origin. In fact, several evidences indicated that MSC could be the most plausible cell of origin. Lately, some works have again pointed the neural crest stem cells (NCSC) as another cell type permissive to the ES fusion transduction.[16-18]

\subsection{The Mesenchymal Stem Cell: Definition of the term}

The stromal cell system has been a clear object of interest over recent years. In fact, there are three main cellular types in the bone marrow: hematopoietic, endothelial and stromal. MSC reside within the marrow and are non-hematopoietic stromal cells characterized by their capacity to self-renew and to exhibit multilineage differentiation. MSC in the human body work as reservoirs of reparative cells, which can be mobilized to the location of 
damaged tissue, differentiating and repairing that damage. These signals might include trauma, fracture, inflammation, necrosis or tumors. Moreover, chemotaxis can also play an important role in the fate of MSC.

MSC were initially isolated from bone marrow and stroma of spleen and thymus and defined as fibroblasts. [19] Later on, bone marrow aspirates were considered to be the most accessible and enriched source of MSC. However, in recent years, MSC have been successfully isolated from cartilage, periostium, synovium, synovial fluid, muscle and tendons. It has also been reported that fetal tissue, placenta, umbilical blood and vasculature also contain MSC.

\subsubsection{Characterization and properties of MSC: immunophenotype, differentiation ability and clinical applications}

The International Society for Cellular Therapy (ISCT) stated that multipotent mesenchymal stromal cells" is the currently recommended designation for the cell type often labeled as MSC. The main problem according to the ISCT is that the particular characterization of MSC is still inconsistent among researchers. Furthermore, many laboratories have isolated and expanded MSC from a large variety of tissues and significant differences were observed between populations.[20, 21]

In order to address this problem three criteria were proposed to help clarify and unify MSC-based research:

i) Capacity to adhere to plastic;

ii) Specific surface antigen expression (Ag expression);

iii) Multipotent differentiation potential. 
MSC must be plastic adherent when maintained in standard culture conditions using plastic culture flasks. Plastic adherence is a well described property of MSC. In terms of Ag expression, at least $95 \%$ of MSC population must express: CD105 (known as endoglin), CD73 (known as ecto 5' nucleotidase) and CD90 (known as thy-1). On the other hand, MSC should not express (<2\%): CD45, CD34, CD14, CD11b, CD19 and the HLA-DR receptor. MSC express numerous important receptors for cell adhesion with hematopoietic cells. These include the integrin families, expression of intracellular adhesion molecules (ICAM) and vascular adhesion molecule (VCAM).[20]

To assure the purity of this population it is recommended to demonstrate the lack of expression of hematopoietic antigens, such as the pan-leukocyte marker CD45 and CD34, which are expressed by the primitive hematopoietic progenitors and endothelial cells. Moreover there are antigens which are mainly expressed by monocytes and macrophages and also B-cell markers such as CD79 $\alpha$ and CD19. MSC are non-immunogenic and so there should not be any expression of HLA-DR molecules under normal conditions.[20]

Finally, MSC should be able to differentiate into various types of cells. When using standard in vitro growth conditions, these should be able to differentiate into, at least, osteoblasts, adipoblasts and chondroblasts (Figure $4)$. 


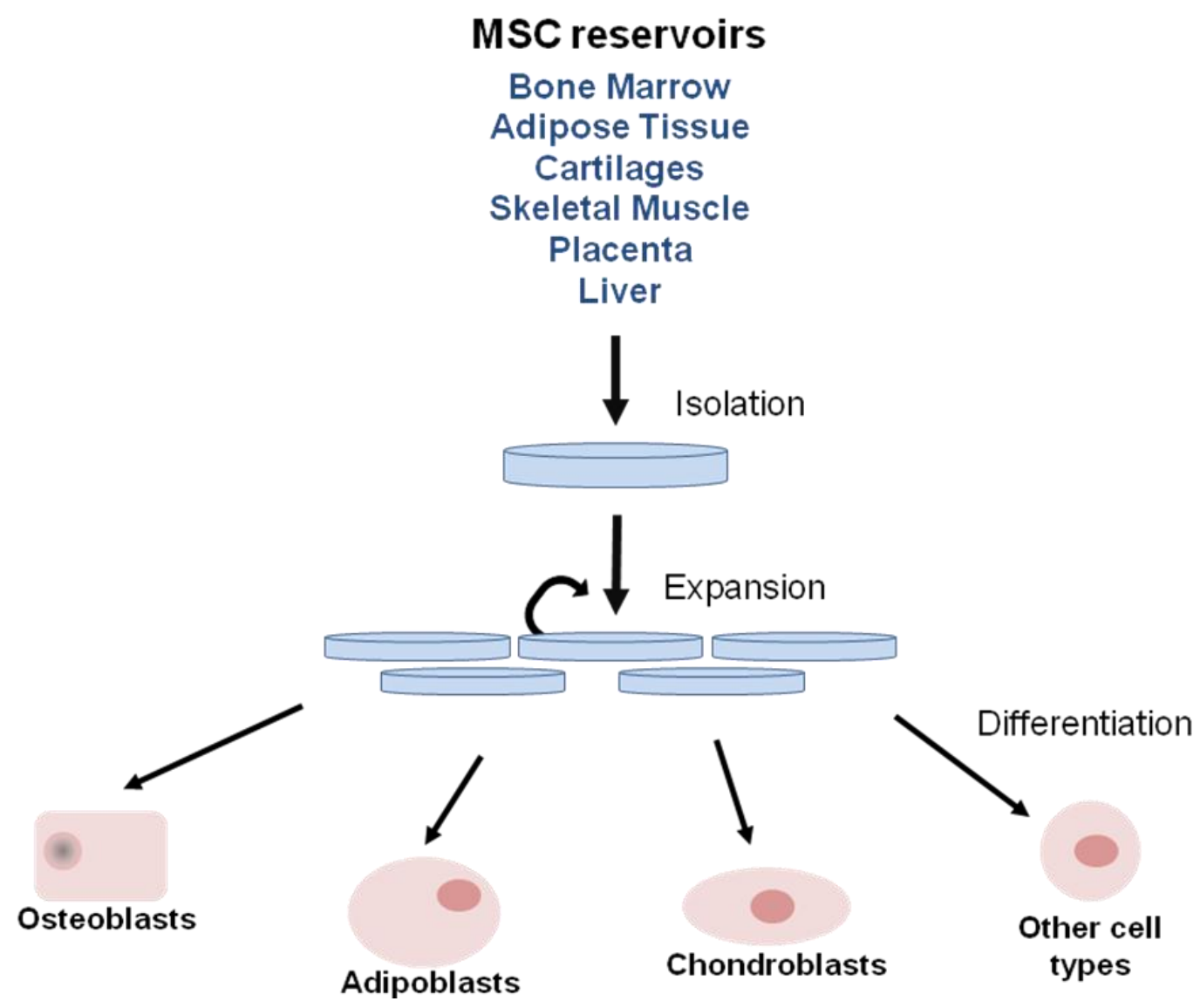

Figure 4. MSC can be isolated and expanded from several tissues and, under the appropriate stimuli, can differentiate into cell types such as osteoblasts, adipocytes, chondrocytes and others (Adapted from Nobela-Arrieta $\mathrm{C}$ et al Nature Review, 2011).

\subsection{The possible implication of MSC in ES initiation: facts and relevance}

The origin of ES has been the focus of intensive research and recently the MSC compartment has been proposed as the most acceptable possibility. $[16,17,22,23]$ In fact, as reflected in Table 1, several studies have focused on MSC as a putative cell of origin of different sarcomas, namely synovial sarcoma, liposarcoma, and ES. 
Table I. Models of sarcoma development using MSC. (Adapted from Rodriguez R et al, Cell Research, 2012).

\begin{tabular}{ccc}
\hline \multirow{2}{*}{ Sarcoma } & \multicolumn{2}{c}{ Oncogenic Events } \\
\cline { 2 - 3 } Mousion gene & $\mathbf{2}^{\text {nd }}$ hit \\
\hline ES & EWSR1-FLI 1 & p53 loss \\
ES & EWSR1-FLI 1 & - \\
\hline Myxoid Liposarcoma & FUS-CHOP & - \\
\hline Myxoid Liposarcoma & FUS-CHOP & p53 -/- \\
\hline Alveolar & PAX3/7-FKHR & p53dn SV40-LT \\
Rhabdomyosarcoma & & \\
\hline \multicolumn{2}{c}{ Human Bone Marrow MSC (NT) } \\
\hline ES & EWSR1-FLI 1 & - \\
\hline Synovial Sarcoma & SYT-SSX1 & - \\
\hline
\end{tabular}

NT stands for no tumors formed upon inoculation in immunodeficient mice

Evidence determined that the accumulation of genetic alterations in longterm cultured MSC led to the development of spontaneous sarcoma-like tumor cells and later transformation into leiomyosarcoma like cells.[24, 25] Accordingly, in liposarcoma, the ectopic expression of the FUS-CHOP chimerical protein coupled to p53 deficiency induced liposarcoma transformation in mice derived MSC.[26] Also, in synovial sarcoma, silencing the SYT-SSX1 fusion protein in tumor cells shifted the regular sarcospheres of tumor cells to a monolayer growth of cells with an increased differentiation potential followed by the expression of MSC genes.[27] Moreover, a recent study performed in osteosarcoma, compared spontaneously transformed MSC with a set of osteosarcoma patients and determined that cdKn2/p16 loss could 
be an important early event in the development of this tumor, thus favoring a probable MSC origin for osteosarcoma.[28] Also in osteosarcoma, the overexpression of the human catalytic telomerase subunit (hTERT) in MSC led to the development of groups of cells with remaining ability to differentiate. Nonetheless, this experiment also demonstrated that despite this pluripotent resemblance to MSC, these cells were highly tumorigenic and presented an expression profile similar to osteosarcoma cells and drastically distinct from the parental MSC.[29]

Particularly in ES, initial data suggested that silencing the EWSR1-FLI1 fusion protein in ES tumor cells resulted in an increased expression of MSC markers as well as in an increased differentiation capacity in the silenced tumor cells. Interestingly, long-term silenced ES cells showed an even increased differentiation potential when compared to short-term silenced ES cells, thus demonstrating that the ES fusion blocks differentiation in ES cells.[16] Accordingly, the knockdown of the ES fusion shifts the expression from ES to an MSC-like profile. [30] Furthermore, the opposite approach, consisting on the ectopic expression of the ES fusion protein in mouse MSC, led to the formation of tumorigenic cells with strong CD99 expression and IGF1 dependency, both intrinsic characteristic of ES cells.[23] Later on, a small group of cells within ES cell lines were characterized as cancer stem cells. This group of cells presented differentiation potential similar to MSC and maintained tumorigenic capacity even when a small number of these cells were inoculated in mice. [31]

Despite the evidence described above, recent data also suggested that NCSC are not only permissive to the expression of the ES fusion, but also able 
to initiate transition to ES-like cells, proving a possible neuro-mesenchymal origin of ES.[18]

\section{Molecular Pathology of ES}

Regarding the Molecular Pathology of ES some particular aspects seem to be crucial to the tumor development and maintenance: the fusion transcript, CD99 over expression, the IGF1-IG1R pathway, and the gain of 1q, specifically focusing on gene PARP1.

\subsection{The ES transcript}

ES is characterized by the presence of aberrant chromosomal translocations that give rise to chimeric proteins, typically involving the EWSR1 gene and one member of the ETS family, as detailed in Table 2.

The most common translocation is produced between the EWSR1 and FLI1 genes, $t(11 ; 22)(q 24 ; q 12)$, leading to the up regulation of direct and indirect gene targets, such as CD99, IGF1,TERT, HSP, CAV1, TOPK among others, and down regulation of IGFBP3 or p21.[17, 32-37] Translocations between the EWSR1 gene and other genes belonging to the ETS family, namely ERG, ETV1, ETV4 or FEV are less frequent in ES.

Some cases, known as "Ewing-like sarcomas", may present EWSR1 fused with non-ETS genes, namely NFATc2, POU5F1, SMARCA5, ZSG, SP3, as well as unrelated translocations to EWSR1 such as CIC-DUX4. Also, very rarely we can find translocations involving genes with functional homology to either EWSR1 such as FUS.[37] 
Table II - Frequency of chromosomal translocations in ES and Ewing-like Sarcomas

\begin{tabular}{|c|c|c|}
\hline Fusion & Chromosomal Translocation & Frequency \\
\hline EWSR1-FLI 1 & $\mathrm{t}(11 ; 22)(\mathrm{q} 24 ; \mathrm{q} 12)$ & $85-90 \%$ \\
\hline EWSR1-ERG & $\mathrm{t}(21 ; 22)(\mathrm{q} 22 ; q 12)$ & $5-10 \%$ \\
\hline EWSR1-ETV1 & $t(7 ; 22)(p 22 ; q 12)$ & $<1 \%$ \\
\hline EWSR1-ETV4 & $\mathrm{t}(17 ; 22)(q 12 ; q 12)$ & $<1 \%$ \\
\hline EWSR1-FEV & $\mathrm{t}(2 ; 22)(q 33 ; q 12)$ & $<1 \%$ \\
\hline \multicolumn{3}{|c|}{ Ewing-like Sarcomas } \\
\hline EWSR1-NFATc2 & $\mathrm{t}(20 ; 22)(q 13 ; q 12)$ & $<1 \%$ \\
\hline EWSR1-POUF51 & $t(6 ; 22)(p 21 ; q 12)$ & $<1 \%$ \\
\hline EWSR1-SMARCA5 & $t(4 ; 22)(q 31 ; q 12)$ & $<1 \%$ \\
\hline EWSR1-SP3 & $t(2 ; 22)(q 31 ; q 12)$ & $<1 \%$ \\
\hline EWSR1-ZSG & $\mathrm{t}(1 ; 22)(\mathrm{p} 34 ; q 12)$ & $<1 \%$ \\
\hline CIC-DUX 4 & $t(4 ; 19)(q 35 ; q 13)$ & $<1 \%$ \\
\hline BCOR-CCNB3 & & $<1 \%$ \\
\hline
\end{tabular}

\subsubsection{Targeting the EWSR1-FLI 1 fusions}

The expression of the EWSR1-FLI1 oncogenic fusion protein in tumor cells, makes it a very attractive candidate in terms of targeted therapy. Despite the common idea that transcription factors are often considered undruggable, recent works have shown that certain compounds, either chemotherapeutic agents or small molecules or peptides, could present certain specificity towards EWSR1-FLI1 and therefore be cytotoxic to ES, namely:

i) Mithramycin ;

ii) YK-4-279 ; 
iii) Ara-C ;

iv) Trabectedin and related compounds.

A high-throughput study which included 50.000 compounds has highlighted mithramycin for its specificity towards cells bearing EWSR1-FLI1 translocations.[38] In vivo and in vitro studies unveiled that mithramycin inhibits EWSR1-FLI1 activity, observed through the deregulation of its target genes after treatment.[38] Mithramycin has demonstrated antineoplastic antibiotic activity, and its security parameters (phase I CT) have already been overcome in other neoplasias. Currently ES patients are being recruited for a Phase I/II CT with Mithramycin in monotherapy for patients bearing the EWSR1-FLI1 transcript (NCT01610570), and other solid tumors in children and adults.

The small molecule YK-4-279, specifically the enantiomer (S)-YK-4.279, has also shown promising results in terms of EWSR1-FLI1 deregulation, mostly by disrupting the bond of this oncogenic protein with the RNA Helicase $A$ $(\mathrm{RHA}) \cdot[39-41]$ This agent blocks the interaction between the transcription factor and $\mathrm{RHA}$, which is apparently relevant in terms of enhancing the oncogenic activity of EWSR1-FLI1.[42] Pre-clinical studies revealed that treatment with YK-4-279 led to apoptosis induction and tumor shrinkage in ES xenografts. [39, 40]

Also, Cytosine arabinose, ARA-C was identified through a highthroughput analysis based on gene-expression. ARA-C is a compound able to attenuate the EWSR1-FLI1 signature.[43] Accordingly, in vitro and in vivo assays showed that ARA-C induces cell death and diminishes tumor growth in xenografts. This fact is mainly justified by the reduction of the EWSR1-FLI1 chimeric protein in ES cells during treatment with ARA-C.[43] Nevertheless, 
another work from Houghton and colleagues evaluated sensitivity to ARA-C on a large panel of pediatric tumor cell lines $(n=23)$ followed by an in vivo assay in ES xenografts. Herein, they observed that in comparison to other tumors, ES cells were not particularly sensitive to ARA-C.[44]

Finally, Trabectedin, also known as ET-743 or Yondelis, is a marine derived compound, currently developed through a semi-synthetic technique, which has been intensively studied for its known anti-tumoral activity. Trabectedin is particularly toxic in SBT.[45-48]

In fact, in myxoid liposarcoma, one example of translocation-related sarcoma, Trabectedin down regulates the binding of FUS-CHOP to promoter regions of genes regulated by this chimerical protein, thus supporting the particular specificity of this agent towards SBT.[45]

Since ES cells are characterized by the presence of transcriptional factors with structural and functional homology to FUS-CHOP, Trabectedin rapidly represented a promising therapeutic approach. Hence, recent observations suggest that Trabectedin may target EWSR1-FLI1 chromosomal translocations present in ES.[49] This study showed that Trabectedin directly interferes with the transcriptional activity of EWSR1-FLI1.[49] Additionally, several studies report this agent as one of the most potent antitumoral agents towards ES, with $\mathrm{IC}_{50}$ of proliferation inhibition on the sub nano-molar range.[47, 49-51]

Resistance to Trabectedin has been related to an increased expression of insulin-like growth factor receptor I (IGF1R) and insulin receptor (IR) as well as an over expression of the efflux bomb Glycoprotein-P.[47] Furthermore, 
EWSR1-FLI1 inhibition, increases cells sensitivity to the action of IGF-1R inhibitors.[17]

Interestingly, a recent manuscript published by Germano et al reports that Trabectedin may also have an important role on angiogenesis inhibition and reduction of tumor-associated-macrophages, hence contributing to tumor shrinkage. This work was based not only on in vitro models, but also on in vivo xenograft models of sarcomas. The study did not include any ES cell line xenograft model or ex vivo samples from patients.[52]

In the clinic, Trabectedin has already finished Phase I CT in pediatric solid tumors (NCT01453283), where the safety in children was determined as well as Phase II CT in pediatric relapsed/refractory ES (NCT00070109). Despite being safe at the tested dosage, it failed as an antitumoral in monotherapy. [53]

\subsubsection{Trabectedin related compounds}

The development of Trabectedin led to the creation of a new group of DNA minor groove binding class of agents related to this drug, which includes PM00104 (Zalypsis) and PM01183 (Lurbinectidin). This particular group of compounds presents a basic common structure composed of two tetrahydroisoquinoline rings $(\mathrm{A}$ and $\mathrm{B})$ responsible for DNA recognition and binding, and a third unit, ring $\mathrm{C}$, which is projected out of the minor groove, thus unable to contact with the DNA, and interacting with nuclear proteins (Figure 5).[48] 


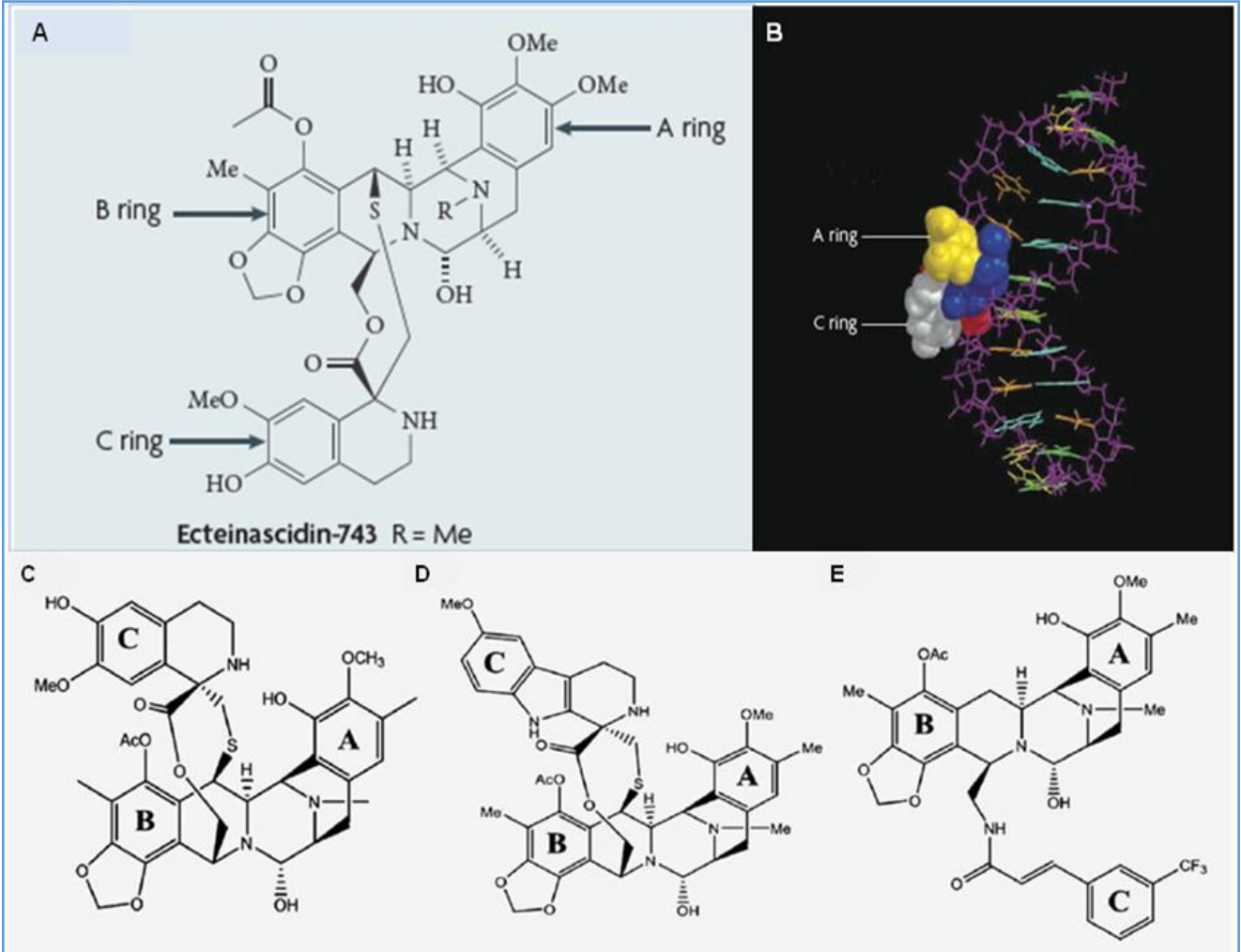

Figure 5. Chemical Structures of Trabectedin and Related compounds. A. Central chemical Structure of Trabectedin is represented on A Rings A and B appear to be similar in all related compound, but changes in ring $C$ confer different pharmacokinetics characteristics. B. Ring $C$ is projected out of the minor groove and impacts response to DNA damage, as observed in 3D chemical structure. C, Trabectedin D. Lurbinectidin. E. Zalypsis chemical structure. D. (adapted from Molinski T et al, Nature Reviews on Drug Discovery, 2009 and Romano M et al, International Journal of Cancer, 2013).

PM00104 or Zalypsis is a new semi-synthetic tetrahydroisoquinoline alkaloid with demonstrated cytotoxic activity towards hematological malignancies and several other tumor cell types in vitro, including ES.[54-56] Initial studies using Zalypsis observed that, like Trabectedin, also this compound is a potent Double Strand Break (DSB) inducer, detected by the presence of $\mathrm{yH} 2 \mathrm{Ax}$ in both NER deficient and proficient cells. Herein, ES cell 
lines TC71 and TC32, presented a slightly higher sensitivity to Trabectedin than to Zalypsis.[54] Another important conclusion of this study had to do with the evaluation of ring $C$ structural value in terms of DNA binding and repair mechanism activation. DNA damage induced by Zalypsis is independent of NER, in contrast to Trabectedin. [54]

A more global study, including 24 cancer cell lines from various solid tumors (prostate, pancreas, ovary, lung, liver, kidney, stomach, colon, bladder and breast) as well as hematological tumors (leukemia cell lines), suggests that Zalypsis is able to bind both naked and methylated DNA (CpG islands), and forms adducts in specific G points of the DNA (GGC;AGC;AGG;TGG;CGG), different from those of Trabectedin (TGG;CGG;AGC;GGC). More importantly, observations from this study also suggest that Zalypsis, despite forming adducts in specific $G$ areas of the double helix, is somehow able to escape NER machinery, probably due to a different $C$ ring structure.[55]

Interestingly, Zalypsis was described as one the most potent cytotoxic agents towards multiple myeloma. A first study showed that Zalypsis was a potent DSB inducer and proapoptotic agent in in vitro and in vivo models of multiple myeloma. In fact, even resistant cell lines to anti myeloma agents were sensitive to this compound. Zalypsis selectively targeted tumor cells while not affecting normal BM stem cell population.[57] Later on, another study observed that this agent was also able to induce cell death by DSB following ineffective DNA damage repair (DDR) selectively in malignant cells (myeloid blast and progenitor cells).[58] 
In the clinic, Zalypsis has already concluded Phase II in relapsed/metastatic ES (NCT01222767) and Phase I in advanced solid tumors/ lymphoma (NCT00359294).

Little is known about Lurbinectedin. Initially known as PM01183, this compound was described as an alkylator agent with a chemical structure similar to Trabectedin (subunits $A$ and $B$ ), where the main differences relayed on ring C. In a first study, Lurbinectidin showed capacity to induce DSB and cell cycle arrest in $\mathrm{S}$ phase in various tumor cell lines (breast cancer, lung carcinoma, colorectal adenocarcinoma/carcinoma, ovarian carcinoma, among others), however, they didn't include sarcoma cell lines.[56]

Lurbinectedin is currently under Phase I CT (recruiting patients) in acute leukemia (NCT01314599) and in Phase II in breast cancer (NCT01525589).

\subsection{CD99: an over-expressed antigen and a target option in ES}

CD99, MIC2, is a $32 \mathrm{KDa}$ cell membrane receptor over-expressed in almost all of ES cells (over 90\%). In normal tissue, a high expression of CD99 can be observed in cortical thymocytes, pancreatic islet cells, granulosa cells of ovary and sertoli cells of testis. In the hematological system, CD34+ present in the bone marrow also show high expression of CD99, especially in all leukocytic lineages.[59-63] In neoplasias, high expression is not exclusive of ES and can also be found in lymphoblastic lymphoma/leukemia and sometimes can also be found in synovial sarcoma, mesenchymal chondrosarcoma and Rhabdomyosarcoma.[62-64]

CD99 has been described as a critical partner in processes such as adhesion, migration, apoptosis and regulation of intracellular membrane protein trafficking. [60, 61, 65-69] This glycoprotein is essential for the appropriate 
differentiation of T-Cells and thymocytes.[59, 60] Also, this protein is critical for the correct diapedesis of leukocytes. CD99 is diffusely expressed on the cell membrane of leukocytes, however, since it's required for the completion of diapedesis to the inflammatory sites, it becomes over expressed and concentrated on the membrane barriers between leukocytes and endothelial cells[61]. Evidence also suggests a link between CD99 and the osteoblastic differentiation, through the transcription factor RUNX2, key in the osteoblastogenesis process.[70]

In osteosarcoma, when CD99 was over expressed, there was a reduction on malignancy with reduction of migration, reduced resistance to anoikis and reduction of the ability to induce tumor formation and metastasis in vivo. In fact, the particular effects on cell migration were later on attributed to the inhibition of ROCK2 activity. [68, 71]

Several works have defined that, together with the ES fusion gene, it represents the most characteristic hallmark on ES tumor biology.[72] Despite this, CD99 over-expression is very unspecific since this feature is shared with other neoplasias. Notwithstanding, CD99 functionality is yet to be fully described. Recent studies have determined that CD99 is crucial for ES malignancy and therefore a potential therapeutic target. ES cells with very high expression of CD99, cell line TC71, were subjected to a stable inhibition of CD99 expression and this lead to a serious of changes in comparison to the parental cell line. In fact, TC71 cells, CD99 depleted, showed a lower anchorage-dependent growth as well as a significant decrease on the capacity to migrate. Moreover the inhibition of CD99 led to a decrease on tumor formation, tumor volume and metastasis formation. Both tumorigenicity and 
metastasis ability were recovered when the CD99 expression was restored. [73, 74]

In fact, evidence has shown that there is a connection between the chimerical protein EWSR1-FLI1 and CD99, mainly due to the presence of miRNA, thus suggesting that CD99 is regulated by the EWSR1-FLI1.[75] Accordingly, CD99 is responsible for maintaining the oncogenic phenotype and differentiation of ES.[73]

Targeting CD99 represents an attractive treatment strategy since it would target almost all of the ES cells. Evidence from Scotlandi and colleagues showed that the inhibition with a monoclonal antibody specific for CD99 leads to cell death through apoptosis.[72, 74] In fact the combination of Doxorubicin and 0662, an anti-CD99 monoclonal antibody, not only increased apoptosis but, most importantly, reduced the tumor formation in mice xenografts.[76]

\subsection{The IGF-1/ IGF-1R axis}

\subsubsection{Clinical relevance and implications}

One of the better described downstream targets of the ES fusion is the IGF-1/ IGF-1R pathway. IGF-1R is a tyrosine-kinase (TK) receptor located in the cell membrane, formed by 4 subunits, two units located on the extracellular part of the cell membrane and other two trespassing the membrane. Indeed the whole system involves several TK receptors: IGF-1R; Insulin receptor (IR) (isoforms $\mathrm{A}$ and $\mathrm{B}$ ), hybrid receptors IGF-1R and IGF-2R as well as ligands: IGF-1, IGF-2 and insulin.[77] Also, the IGF binding proteins (IGFBPs) play an important role blocking IGF-1 and IGF-2 from activating the respective receptors, whenever suitable. In physiological conditions the TK receptor IGF- 
$1 \mathrm{R}$ and its ligand, IGF-1, are critical in terms of cell growth and accurate cell differentiation.

Activation of IGF1R occurs by reversible binding of IGF-1 which can lead to receptor dimerization and activation of the neighbor IGF-1R, therefore enhancing pathway activation. Immediately, a cascade of downstream targets is activated (Figure 6). The IGF-1R cascade can also be enhanced by internalization of activated TK receptor signaling through the cytoplasm in caveolin pits of clathrin coated vesicles, as described below.[78]

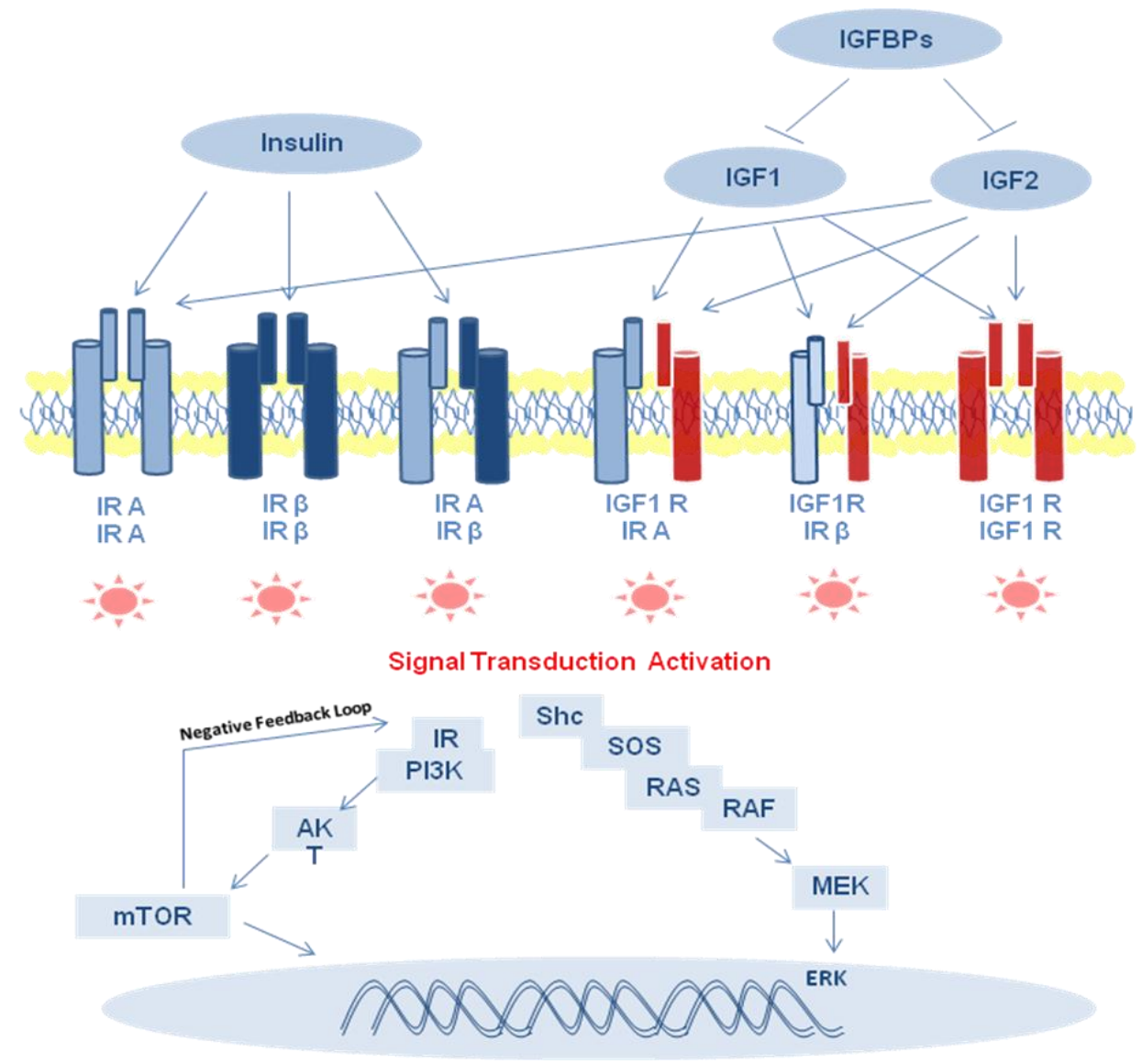

Figure 6.The IGF-1/ IGF-1R downstream pathway. (Adapted from Michael Pollak, Nature Reviews on Cancer, 2012 and Michael Pollak et al, Nature Reviews on Cancer, 2004). ES cells are able to witch from and IGF1-IGF1R dependency to an IGF2-IR dependency. Linsitinb is a dual inhibitor of IGF1R and IR, thus overcoming the problem of anti-IGF1R acquired resistances. 
IGF-1R targeting represents an attractive strategy in ES since it is a known up-regulated downstream target of EWS-FLI-1. Particularly, it has been observed that in vitro, that ES cells show reduced expression of IGFBP-3 with over-expression and constitutive activation of IGF-1R downstream targets.[22, 79-81] Interestingly, ex vivo studies showed that among 290 samples, $70-80 \%$ of ES patients presented high expression of IGF-1R and also of IR. A mere $10 \%$ were IGF-1R negative, showing high IR expression.[82]

Several efforts are currently being carried out, either through inhibition of IGF-1R with small molecules or monoclonal antibodies (MAb), humanized (HAb) or fully human. In ES, inhibition of the IGF-1R pathway has an important pre-clinical effect; nonetheless, up to today clinical impact of IGF-1R inhibition remains poor, thus stressing the need to develop distinct, or more suitable, therapeutic approaches to inhibit this pathway.[83-85]

\subsubsection{Anti-IGF1R Monoclonal Antibodies}

MAbs have been clinically developed in mice, initially there were $\mathrm{HAb}$, IgG1MAb (Human-Mouse-Human) and recently fully Human IgG1; IgG2 or IgG4. Administration of MAbs is surrounded by highly safety measures in order to prevent immunogenic responses. Among MAbs, the Fully Human MAbs represent a safer approach in comparison to Humanized MAbs which increase the probability of side effects. Anti-IGF-1R MAbs mechanism is based not only on the obvious antagonism with IGF-1, inhibiting IGF-1R, but also by the later down-regulation of the IGF-1R cascade. In fact, several anti-IGF-1R MAbs namely: AVE1642, Figitumumab, Cixutumumab, Robatumumab have already shown pre-clinical and/or clinical efficacy in either ES or the broad group of sarcomas.[86] These compounds have been brilliantly described in a recent 
review from Olmos $D$ and colleagues; therefore, here we will be describing the most representative MAbs with preclinical or clinical evidence in ES.[87]

AVE1642 is a HAb IgG1, anti-IGF-1R MAb, with in vitro demonstrated activity towards ES cells.[88, 89] ES cells resistance to this agent has been related to neural differentiation and angiogenesis.[88] Outcome from Phase I CT of AVE1642 in patients with advanced solid tumors, which didn't include ES patients, determined good tolerability of this agent in combination with docetaxel and gemcitabine.[90]

Figitumumab (CP-751,871) fully human IgG2 anti-IGF-1R MAb has shown preclinical evidence towards ES and has already passed Phase I and II CT in patients with refractory ES (NCT00560235). According to preclinical evidence, resistance to Figitumumab is related to angiogenesis.[88] Figitumumab entered Phase I CT which included 15 patients with refractory ES. The phase II study including 107 ES patients showed modest antitumor activity in monotherapy.[91] In this set of patients, high IGF-1 serum levels, correlated with poor prognosis and since both Figitumumab and IGF-1 show very similar affinity towards IGF-1R, the direct competition between high levels of ligand and MAb impaired figitumumab-IGF-1R blockage.

Cixutumumab (IMC-A12) is also a fully human MAb, with demonstrated in vitro activity in various tumor types, namely head and neck squamous cell carcinoma (HNSCC) and childhood malignancies including neuroblastoma, Wilms tumor and sarcomas (Rhabdomyosarcoma; ES and osteosarcoma).[67, 92] Preclinical evidence reported by the Pediatric Preclinical Testing Program (PPTP) concluded that Cixutumumab was an active intermediate cytotoxic agent. However only one ES xenograft out of five was evaluable for its 
activity.[67] In HNCSS, cells showed a co-expression of IGF-1R and EGFR. Treatment with Cixutumumab blocked IGF-1R which resulted in the induction of mTOR and AKT expression. When Cixutumumab was combined with Rapamycin which caused inhibition EGFR and mTOR respectively, antitumor effects were reduced. These findings suggested that resistance to anti-IGF-1R MAbs might be related with induction of AKT/mTOR.[92] In fact, also under the PPTP of the Cixutumumab and Rapamycin combination was studied. Among the set of mice xenograft models studied here (ES, osteosarcoma and rhabdomyosarcoma), ES showed the most dramatic response to the combinatory regimen, with a longer time to progression and clear synergistic effects.[93] Moreover, Cixutumumab is currently in Phase II CTs for advance sarcomas, where metastatic /advanced ES represent one of the arms of this study (NCT00668148).

Robatumumab (R1507) is another anti-IGF-1R MAb with preclinical and clinical evidence towards ES. ES cell lines showed sensitivity to R1507, although an siRNA targeting EWS-FLI1 fusion protein was able to induce higher levels of apoptosis that the MAb.[94] In the clinic, a Phase II study of R1507 in Patients with recurrent or refractory ES included 132 patients and resulted in $10 \%$ of overall response for $>6$ months. Outcome from this study demonstrated that R1507 has modest activity as a single agent; some reasons which could explain this failure include for instance lack of pre-screening of IGF-1R: IR ratio in the ES patients enrolled in this study. Also, no drug combination was administered.[95] 


\subsubsection{Small molecules}

Various small molecules have been developed as a way to selectively inhibit IGF-1R by ATP competition, namely Novartis compounds NVP-AEW541 and NVP-ADW742. Recently, a new small molecule named Linsitinib, previously known as OSI-906, has been shown to inhibit both the IGF-1R and $\mathrm{IR}$, thus representing a clear benefit in terms of escaping drug resistance.[88, 89]

AEW541 and ADW742 have demonstrated in vitro and in vivo activity towards ES.[88, 96, 97] Our group previously confirmed the toxic activity of ADW742 and AEW451 in vitro and in vivo, alone and in combination with the HSP90 inhibitor 17AAG.[78]

The new small molecule Linsitinib demonstrated toxic activity in ES cells, with the particularity of inhibiting specifically IGF-1R/IR, and thus blocking the auto-loop previously described in which ES cells switch from IGF-1/ IGF-1R dependency to IGF-2/ IR dependency given the homology between both these receptors. Linsitinib has demonstrated in vitro and in vivo activity in ES. [88] Also, Linsitinib activity is being evaluated under the Phase I/II CT named LINES in ES patients with recurrent/disseminated disease.

Interestingly, in vitro systems show that ES cells-resistant to AVE1642, Figitumumab and AEW541 were able to maintain their survival and proliferation ability by switching from IGF-1/ IGF-1R pathway to IGF-2/ IR-A pathway. In fact, there is evidence that the IGF-1R: IR ratio might very well be the hidden responsible for resistance to treatment with IGF-1R inhibitors (MAbs or small molecules). This fact could justify why patients with higher IR levels don't respond to anti-IGF-1R therapies whereas patients with higher IGF-1R: IR ratios 
respond better to this targeted therapy. Herein lays the peculiarity of the dual inhibitor Linsitinib which might represent an advantage to other IGF1R inhibitors, given that it indistinctively blocks both receptors.

\subsection{PARP1 and PARP Inhibitors: General Review and facts in ES}

PARP1 is an enzyme of the family of the poli-(ADP-ribose) polymerases. This gene, located on chromosome 1 (1q41-q42), is up-regulated in some human cancers and it's over expression is often related with chemo resistance. Recently, a retrospective study revealed the prognostic value of the gain of the long-arm of chromosome 1 (1qG) in ES.[10]

PARP family of proteins includes several members namely, PARP1, PARP2, PARP3, PARP4, PARP5a, PARP5b. Among them, PARP1 and PARP2 physically interact with each other showing redundant functions. They are the only known members of the PARP family who are activated by union to DNA.[98, 99] PARP proteins catalyze a type of post-transductional modification that entails the addition of ADP-ribose groups to the acceptor proteins using NAD+ as a donor substrate of ADP-ribose groups and forming of poli-ADPribose biopolymers (PAR). In response to stress situations, which cause DNA damage, PARP proteins are up-regulated and the generated biopolymer PAR recruits other members of DDR system such as the XRCC1 factor and the DNA-ligase III to the damaged site. In turn, these proteins are poli-ADP ribosilated and consequently activated, favoring the signaling and activation of DDR. [98] Besides the relevant role in DDR, PARP family also participates and contributes to other processes, namely:

i) The maintenance of the genomic stability;

ii) Chromatin remodeling; 
iii) Transcription;

iv) Cell cycle and cell death regulation.

v) Angiogenesis.

The first concept to introduce regarding PARPi is the concept of synthetic lethality. Synthetic lethality arises when a combination of mutations in two or more genes leads to cell death through apoptosis, whereas a mutation in only one of these genes does not. A synthetic lethal genetic screen, it begins with a mutation that, although unable to kill the cell, confers a particular phenotype and then systematically test other mutations to determine which one confers lethality. For instance, it happens with BRCA genes, which usually regulate homologous recombination (HR) machinery, and PARP1 which plays a main role in base excision repair (BER). The use of PARPi in tumors with defects in BRCA genes has been largely explored. $[98,100,101]$

Apart from monotherapy, PARPi can also play the role of adjuvant, sensitizing tumor cells to chemotherapeutic agents inducing single strand break (SSB) and DSB, particularly in tumors harboring mutant genes in DDR machinery.

The role of PARP1 in tumors with ETS-fusions, including ES, has also been recently shown.[102, 103] Recent in vitro and in vivo studies have shown that ES cells are sensitive to treatment with the PARPi Olaparib when combined with the alkylator agent Temozolamide.[102-104] This drug combination, practically eliminated tumor growth in a xenograft model of ES. Another work related ES cells sensitiveness to Olaparib with a possible interaction between PARP1 and the ES transcript.[102] Given the importance of these preclinical results, a Phase II CT was initiated in metastatic/ recurrent ES Patients as a 
second line treatment (NCT01583543). This study was stopped recently as preliminary data suggested that Olaparib appears to be insufficient in monotherapy, strongly suggesting combination therapy.

\section{Overview on the DNA Damage and Repair pathway}

\subsection{DNA Damage induction}

Cancer cells are characterized by a deregulated cell cycle with an unbalanced growth-death ratio. Consequently, agents that induce DNA damage or inhibit molecules involved in DDR represent an important therapeutic strategy in cancer therapy. Damage in DNA can be caused either directly or indirectly. Radiation, for instance, generates direct damage; however some agents such as topoisomerase inhibitors generate damage on an indirect way. DNA damage could affect one strand of the double helix, or both strands. It is important to notice that DSB represents the most lethal way of DNA damage.

In the complex global context of DDR, molecules can be organized by signaling; mediators and effector molecules. DSBs, for instance, generate a signaling cascade which activates mediator molecules and these finally recruit effector molecules to repair DNA damage. If damage is repaired, cells carry on with their cycle, however when the damage is irreparable, cells trigger programmed cell death: apoptosis. UV radiation and chemical agents generate adducts with particular sections of the double strand causing the DNA to bend and break on both strands, hence DSB. DSBs is the sort of DNA damage most lethal to cells. 


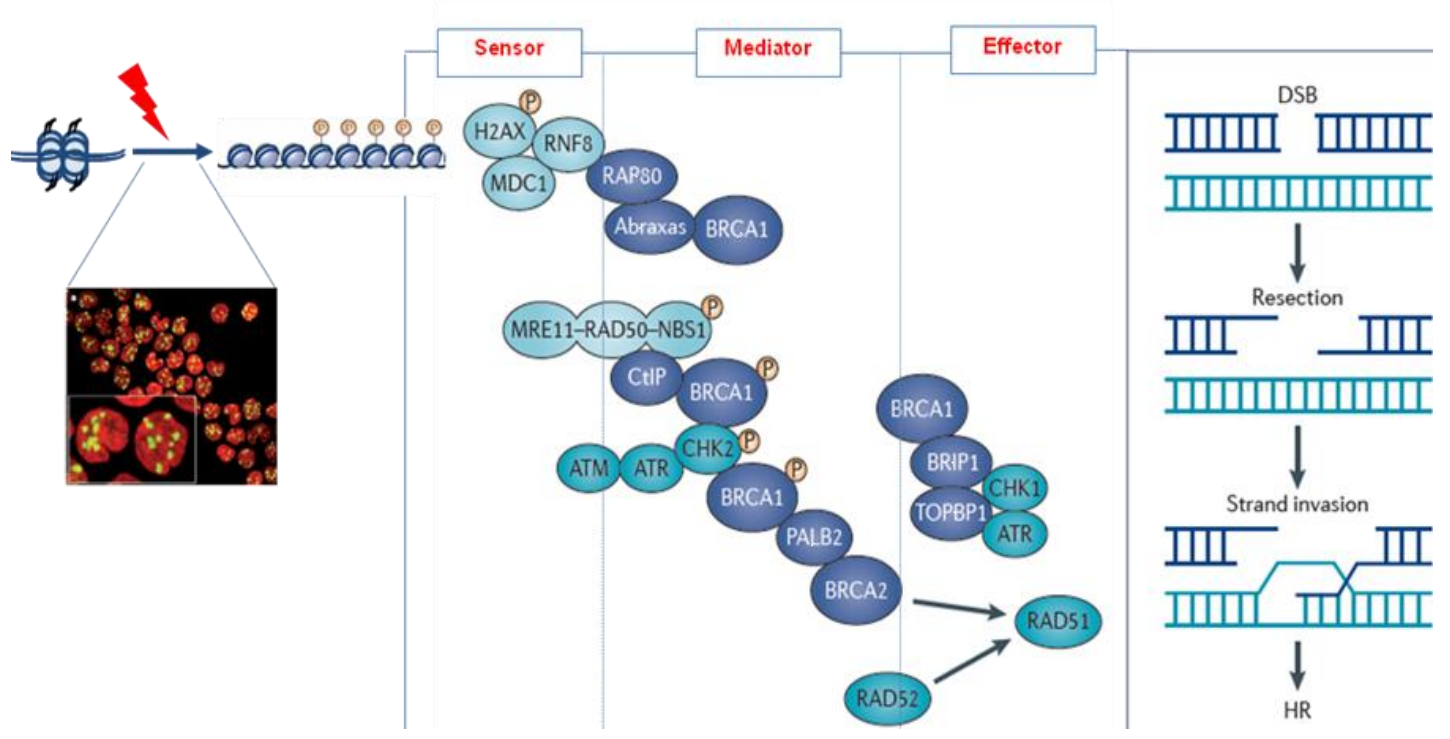

Figure 7. Simplified schematic view of DNA damage induction, with $\mathrm{H} 2 \mathrm{AX}$ phosphorylation, presence of intra-nuclear foci of $\mathrm{\gamma H} 2 \mathrm{AX}$ and further activation of sensor/mediator/effector molecules with the final goal of DNA repair by homologous recombination (HR) (adapted from Rohini Roy et al, Nature Reviews on Cancer, 2012 and William M Bonner et al, Nature Reviews on Cancer, 2008).

The initial marker of this sort of damage is the phosphorylation of histone $\mathrm{H} 2 \mathrm{AX}$ on serine 139, and the formation of phosphorylated foci in the damaged surrounding areas. In the heterochromatin state (DNA condensed + core of four histones), genes are inactive. When DNA damage is induced the tails of H2AX are phosphorylated and the core of histones opens DNA, exposing the DNA rupture to the repair mechanisms. The DNA damage is therefore surrounded by the $\mathrm{pH} 2 \mathrm{AX}$ foci. Several works focusing on this particular signaling mechanisms have concluded that the presence of $\mathrm{pH} 2 \mathrm{AX}$ foci, each one containing hundreds of phosphorylated histone, is directly proportional to the DSB that have been induced. This is considered the first molecule on the path of DNA damage by DSB (Figure 7). 


\subsection{Activation of DDR pathways}

Physiological pathways include HR; non-homologous end joining repair (NHEJR), and NER, BER and mismatch repair (MMR). Different anti-tumoral agents activate distinct pathways to repair the damage, as reflected in Figure 8. NER and BER are responsible for the repair of SSB. In contrast, DSB activates HR and NHEJ pathways.

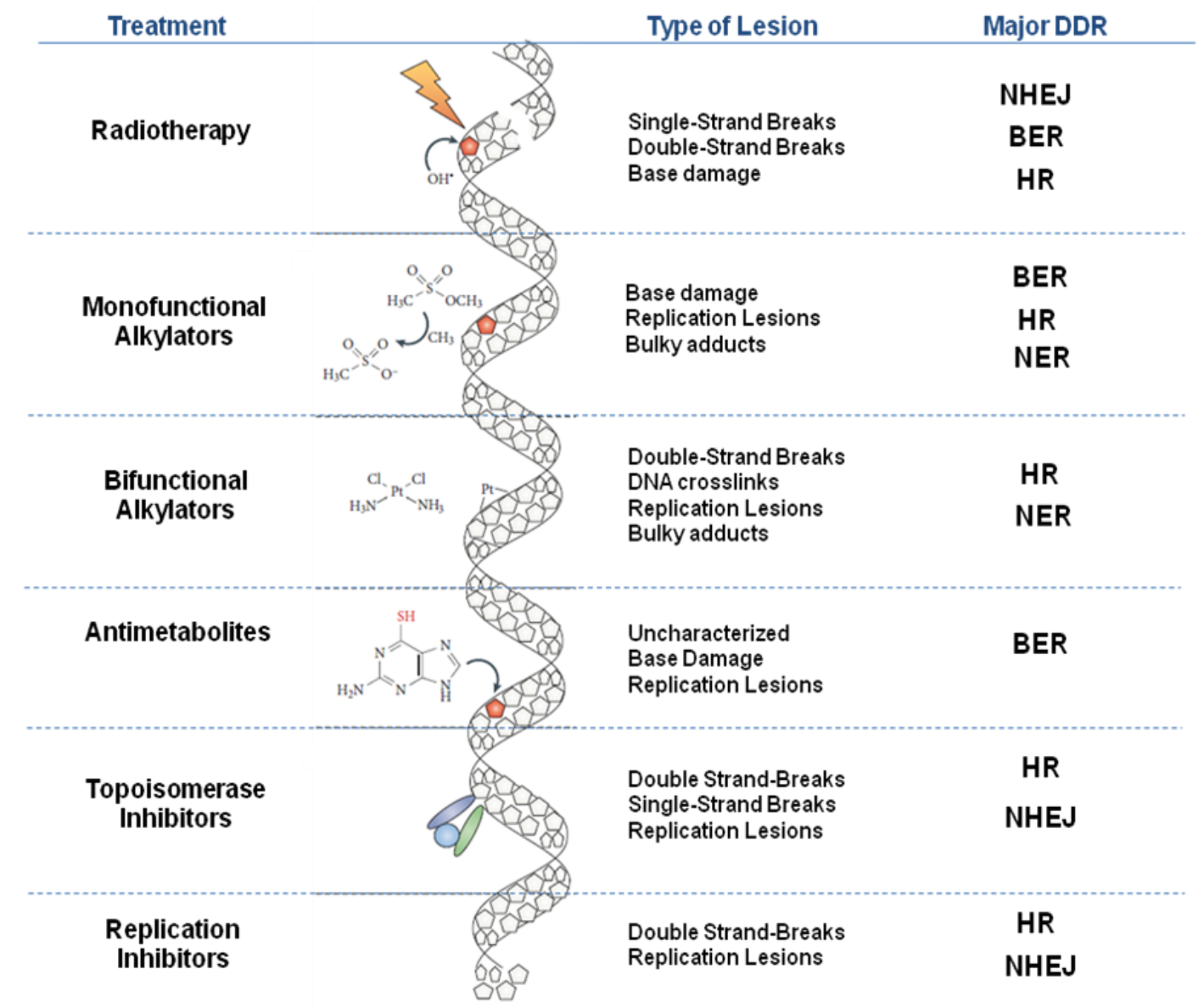

Figure 8. Graphic Representation of DNA damaging agents and the type of lesion induced as well as the major repair pathways activated by each group of drugs. (Adapted from Helleday T et al, Nature Reviews on Cancer, 2008. Main pathways: DSB, double strand breaks; NHEJ, Non-homologous end joining repair; BER, base excision repair; HR, homologous recombination; NER, nucleotide excision repair). 
DNA repair mechanisms are dynamic models in constant change. SSB often activates NER and BER machinery. BER removes defective DNA bases, for instance via DNA-glycosylases, (8-oxoguanine DNA glycosylase 1 (OGG1)), which are then hydrolyzed by APE1, an apurinic-apyridimic endonuclease. Here, PARP1 plays an active role recruiting repair enzymes, as it was explained above. In fact, PARP1 can bind to both SSB and DSB, yet it appears to be more relevant in SSB and unnecessary for HR.

NER pathway is able to recognize damage induced by UV light. This damage generates bulky adducts. Adducts are removed and repair is performed using the single unaffected DNA strand as a model. NER includes proteins such as xeroderma pigmentosum family of proteins (XPA, XPC and XPG) and ERCC1.

DSB on the other hand can be repaired by HR or NHEJ. NHEJ repair machinery is mostly active during G1 phase of the cell cycle and includes proteins such as Ku70-Ku80 heterodimers which bind to the damaged site and recruit DNA-PKcs (DNA-dependent protein kinase catalytic subunit). These can phosphorylate H2AX. While Artemis processes the DNA ends, DNA ligase 4 (Lig4) unites both ends and XRCC4 stabilizes the complex. Finally, 53BP1 (p53 binding protein 1) rapidly accumulates on the surrounding areas of $y \mathrm{H} 2 \mathrm{AX}$ foci. It appears to have an important role in balanced competition between HR and NHEJ.

$\mathrm{HR}$ is a highly complex mechanism active in late $\mathrm{S}$ or G2 phases of the cycle and as an important different with NHEJ, it is described as an error-free mechanism of repair, in contrast to NHEJ This pathway includes molecules such as ATM; BRCA1; BRCA2; RAD51 and RAD52. ATM and ATR kinases 
amplify and stabilize $\mathrm{\gamma H} 2 \mathrm{AXfoci}$. BRCA1 is activated via phosphorylation by ATM-ATR kinase, and represents a central molecule in HR. BRCA1 activates the dimer BRCA2-RAD51. RAD51 recombinase and RAD52 are the final effector molecules and responsible for restoring genetic information. RAD51 is crucial for cell survival. ATR also phosphorylates CHK1 which leads to the activation of molecule RAD51 (Figure 9).

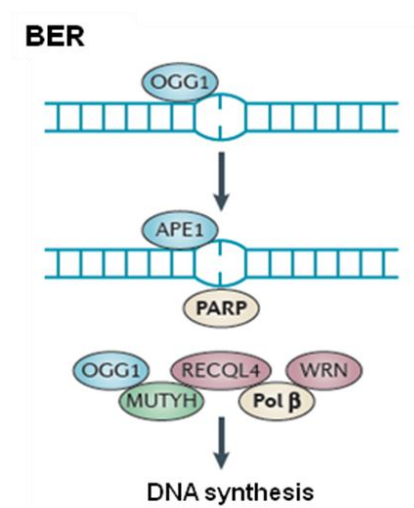

NER

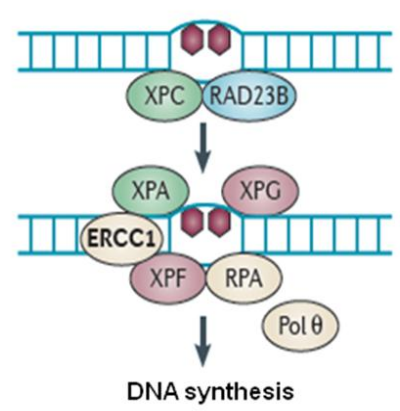

HR

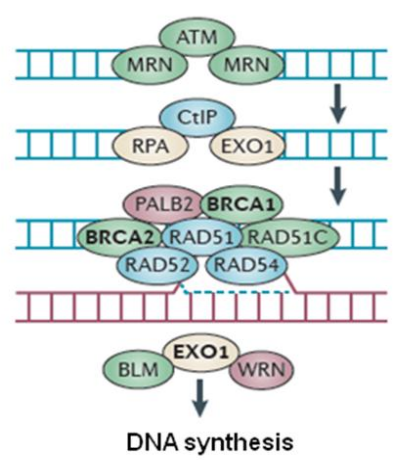

NHEJ

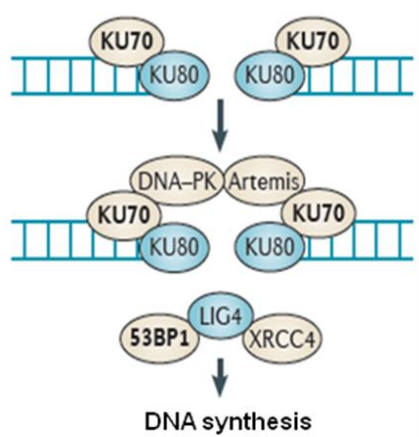

Figure 9. Schematic view of BER, HR, NER and NHEJ, DNA repair pathways and molecules involved. Adapted from Peter Bouwman and Jos Jonkers, Nature Reviews on Cancer, 2012.

If on the one hand agents such as Trabectedin, Zalypsis and Lurbinectedin directly induce DNA damage, on the other hand Olaparib is an indirect DNA damaging agent since it is main activity is to inhibit PARP. 
Each one of these agents interferes with specific pathways of DDR, as depicted on the following table.

\begin{tabular}{|c|c|c|}
\hline Drug & Type of Lesion & DDR \\
\hline Trabectedin & Direct: DSB & HR ; TC-NER \\
\hline Zalypsis & Direct: DSB & HR \\
\hline Lurbinectidin & Direct: DSB & HR \\
\hline Olaparib & Indirect: SSB ; DSB & BER \\
\hline
\end{tabular}

\subsection{Trabectedin: crosstalk with NER and further DDR activation}

Trabectedin presents a unique mechanism of action, creating adducts with guanine rich regions of the minor groove of the DNA and then bending towards the major groove finally disrupting the double helix.[48, 105-107] This particular mechanism gives rise to a very unique DDR. As expected, Trabectedin interacts with NER machinery, but the induced damage appears to be repaired exclusively by HR machinery. Several studies performed in NER proficient/deficient cells as well as HR deficient cells observed that Trabectedin is highly cytotoxic in HR deficient cells, and less toxic in NER deficient cells than in NER proficient cells. Surprisingly, the chemical structure of Trabectedin allows ring $\mathrm{C}$ to generate complexes with NER (TC-NER). These complexes induce DNA strand breaks which later activate HR molecules BRCA1 and BRCA2. Regarding NHEJ machinery, it appears to have a minor influence regarding treatment with Trabectedin since cells deficient in NHEJ show a slightly decreased sensitivity (4 fold) to Trabectedin. [54, 108] Nevertheless the role of NHEJ in Trabectedin induced damage is yet uncertain as some works 
describe that deficiencies in Ku80 and DNA-PK show no differences when compared to parental cell lines in terms of sensitivity. [106]

Altogether, HR appears to have a higher role on Trabectedin damage repair than NHEJ. Moreover, NER forms complexes with Trabectedin, increasing the toxic activity of the compound.[105, 107]

\subsection{Trabectedin related compounds and DDR activation}

Initial studies using Zalypsis observed that, similarly to Trabectedin, this compound was also a potent DSB inducer. This fact was detected by the presence of $\mathrm{\gamma H} 2 \mathrm{Ax}$ in both NER deficient and proficient cells, as well as in ES cells. Herein, ES cell lines TC71 and TC32, presented a slightly higher sensitivity to Trabectedin than to Zalypsis. [54] Another important conclusion of this study was relative to the evaluation of ring $C$ structural value in terms of DNA binding and repair mechanism activation. Apparently, DNA damage induced by Zalypsis is independent of NER, in contrast to Trabectedin, and is repaired by HR.

Some very recent studies deepen on the mechanism of action of Lurbinectedin, mainly in terms of DNA binding and DDR induction, in comparison to Trabectedin. In fact, a work published by Soares et al, showed that when using Platinum resistant cells, both Trabectedin and Lurbinectedin showed unchanged sensitivity in comparison to the parental cell lines. These cell lines developed their mechanism of resistance by over expressing NER components.[109] This fact led to the conclusion that platinum agents such as oxiplatin and cisplatin induce repairable DNA damage, NER-dependent, in contrast to Trabectedin and Lurbinectedin. Interestingly, this study also concluded that although DNA lesions induced by treatment with Trabectedin 
and Lurbinectedin fail to recruit NER machinery with the objective of DDR both compounds interact with NER proteins increasing their intrinsic toxic activity. [110] Moreover, cell models with deficient HR presented higher sensitivity to treatment with Lurbinectedin. As a conclusion, DNA damage induced by Lurbinectedin is very similar to Trabectedin: forming toxic-complexes with NER and activating HR.

\subsection{Olaparib and DDR activation}

Regarding PARPi Olaparib, intensive studies mainly in breast cancer cell lines and breast cancer samples have already illustrated and clarified the mechanism of action in terms of DNA damage induction.

There are several known PARPi, such as Olaparib or Veliparib. Most of them compete with NAD+ substratum interfering binding to the active site of the enzyme, a region highly conserved among the different members of the PARP family.[98-100] The use of PARPi in monotherapy for breast cancer in BRCA1 and/or BRCA2 defective tumors is probably the best example of synthetic lethality in the clinic.[111] The loss of heterozygosis in BRCA1 or BRCA2 tumor suppressor genes provokes a strong deficiency in HR machinery, inducing genomic instability which leads to the appearance of tumors. Given that this HR loss of function is not observed in normal cells, PARPi can be used as a selective cytotoxic in BRCA1 and/or BRCA2 defective tumor cells through the following chain of events. Administration of PARPi leads to more and more irreparable SSB, given that PARP is not active and able to repair them. Consequently the replication fork collapses leading to DSB. In turn, these DSB which should be repaired by HR effectors, can only be repaired by the NHEJ system, which also induces genomic instability and finally cell death.[98] 
Therefore, the possibility of using PARPi in tumors with serious defects in HR components is of obvious great clinical interest. [98, 101] However, more recently, the mechanism of PARP/BRCA synthetic lethality described above has been challenged, stressing the role of PARP1 in replication fork stability.[112]

PARPi are effective agents in therapy not only in monotherapy but also combined with other agents. In fact, combination with agents able to induce DNA damage, such as Temozolamide, DXR or Trabectedin, represent a promising approach in the clinic. 
The Pathogenesis of Ewing Sarcoma| Introduction 
Hypothesis and Objectives 
The Pathogenesis of Ewing Sarcoma| Hypothesis and Objectives 


\section{Hypothesis and Objectives}

The phenotypic study of markers such as CD99 in MSCs from ES patients as compared to MSCs from HD and to ES cells will unveil actionable therapeutic targets. IGF1R/IR and PARP1, potential therapeutic targets, can be challenged with specific drugs both in monotherapy and combined with drugs which induce DNA damage, such as Trabectedin and Trabectedin related compounds, leading to tumor growth inhibition.

This hypothesis can be proved through these objectives:

1. Evaluation of CD99 and other pathogenetic markers such as CD271 and CD117 in MSC derived from ES Patients in comparison to ES cell lines and ES samples, and study of anti-CD99 toxicity in MSC and ES cell lines.

2. Evaluation of Trabectedin and Trabectedin-related compounds in ES cell lines. Study of the mechanism of toxicity in ES cells.

3. In vitro an Ex vivo study of Trabectedin effects on the IGF1R pathway. Assessment of Linsitinib toxicity alone and in combination with Trabectedin.

4. Evaluation of Parp1 expression and toxicity of PARPi: Iniparib, Veliparib and Olaparib in ES cells. Deep In vitro and In vivo study of the combination of Trabectedin with PARPi. 
The Pathogenesis of Ewing Sarcoma| Hypothesis and Objectives 


\section{Material and Methods}


The Pathogenesis of Ewing Sarcoma/ Material and Methods 


\section{Cell Lines and samples}

\subsection{Tumor Cell lines characterization and long-term culture}

ES cell lines, TC71, A4573, TTC466, SK-ES-1 and STAET2.1 were cultured in Roswell Park Memorial Institute (RPMI) medium (Gibco ${ }^{\circledR}$ - Life Technologies-Invitrogen; Paisley, UK) with $10 \%$ fetal bovine serum (FBS) $\left(\right.$ Gibco $\left.{ }^{\circledR}\right)$. A673 cell line was cultured in Dulbecco's modified Eagle's-low glucose (DMEM) medium (Gibco ${ }^{\circledR}$ ) supplemented with 10\% FBS. SKNMC; STAET1; RDES; RM82; WE68; CADO-ES and SJRH were cultured in RPMI medium supplemented with $10 \%$ FBS. All media were supplemented with $1 \%$ Glutamine $\left(\right.$ Gibco $\left.^{\circledR}\right)$ and 1\% Penicillin/Streptomycin (P/S) $\left(\right.$ Gibco $\left.^{\circledR}\right)$. With the exception of A4573; SJRH and TTC466, all cell lines have been previously characterized within the EUROBONET consortium.[113] All ES cell lines were grown in plates pretreated with gelatin (Sigma Aldrich) for at least 3 hours.

TC-ET 12nM, resistant cell line, kindly provided by Dr. Katia Scotlandi, IOR (Istituti Ortopedici Rizzoli (IOR), Italy), was cultured in complete RPMI (10\% FBS, $1 \%$ glutamine and $1 \% \mathrm{P} / \mathrm{S}$ ) with $12 \mathrm{nM}$ of Trabectedin.

Breast cancer cell lines, MCF7, HCC-1937, MDA-MB-231 and MDA-MB436 were cultured in DMEM 10\% FBS, $1 \% \mathrm{P} / \mathrm{S}, 1 \%$ Glutamine. MCF7 is BRCA1 ${ }^{+/+}$, HCC-1937 is BRCA1 ${ }^{-/}$, MDA-MB-231 is BRCA1 +/- and MDA-MB437 is BRCA1-/-, were kindly provided by Pharma Mar, Madrid, Spain. 
Table IV. Cell lines characterization

\begin{tabular}{cccc}
\hline Cell line & Pathology & Fusion type & TP 53 status \\
\hline A4573 & Ewing Sarcoma & EWS-FLI 1, type 3 & ${ }^{* \star}$ \\
\hline A673 & Ewing Sarcoma & EWS-FLI 1, type 1 & 2 bp ins.codon 119 \\
\hline TC71 & Ewing Sarcoma & EWS-FLI 1,type 1 & arg213stop \\
\hline RDES & Ewing Sarcoma & EWS-FLI 1,type 2 & arg273stop \\
WE68 & Ewing Sarcoma & EWS-FLI 1,type 1 & Wild type \\
\hline ES CADO & Ewing Sarcoma & EWS-ERG & Wild type \\
\hline SK-ES-1 & Ewing Sarcoma & EWS-FLI 1, type 2 & cys176phe \\
\hline STAET 1 & Ewing Sarcoma & EWS-FLI 1, type 1 & Wild type \\
\hline RM82 & Ewing Sarcoma & EWS-ERG & arg273his \\
\hline SKNMC & Ewing Sarcoma & EWS-FLI 1, type 1 & del EX2-4 \\
\hline TTC466 & Ewing Sarcoma & EWS-ERG & ${ }^{* *}$ \\
\hline SJRH & Rhabdomyosarcoma & - & ${ }^{* *}$ \\
\hline STAET 2.1 & Ewing Sarcoma & EWS-FLI 1, type 3 & cys277tyr \\
\hline
\end{tabular}

** stands for unknown

\subsection{Mesenchymal Stem Cell isolation, expansion and long-term culture}

BM was harvested by aspiration from the iliac crest from ES patients under general anesthesia, having previously obtained oral or written informed consent. If the primary tumor was located in the iliac bone, the contra-lateral site was used for BM harvesting (Table V). BM mononuclear cells were isolated by Ficoll density gradient separation. Washed cells were resuspended in DMEM medium $\left(\right.$ Gibco $\left.^{\circledR}\right)$ supplemented with $10 \%$ FBS and $1 \% \mathrm{P} / \mathrm{S}$.

Cells were plated at a density of $16 \times 10^{4} / \mathrm{cm}^{2}$. Cultures were maintained at $37^{\circ} \mathrm{C}$ in a humidified atmosphere containing $5 \% \mathrm{CO}_{2}$ in $75 \mathrm{~cm}^{2}$ flasks. When cultures reached approximately $70 \%$ confluence, cells were detached by treatment with 
trypsin/EDTA $\left(\right.$ Gibco $\left.^{\circledR}\right)$ and replated at a density of $4000 \mathrm{cells} / \mathrm{cm}^{2}$, nonadherent cells were discarded. Cells were divided up to a maximum of 6 times. When sufficient MSC were expanded, cells were harvested and cryopreserved in culture medium supplemented with $10 \%$ dimethyl sulfoxide.

Table V. Characterization of BM-MSC from ES Patients

\begin{tabular}{lccccc} 
Sample & PN & Age & Fusion type & Primary tumor & Remarks \\
\hline ES-P-01 & P1 & 12 & EWS-FLI1 & Humerus & - \\
\hline ES-P-02 & P2 & 15 & EWSR1 BA positive & Intramuscular & - \\
\hline ES-P-03 & P3 & 18 & EWS-FLI1 & Humerus & - \\
\hline ES-P-05 & P1 & 17 & EWS-FLI1 & lliac crest & - \\
\hline ES-P-07 & P2 & 20 & EWSR1 BA positive & Fibula & - \\
\hline ES-P-08 & P2 & 17 & EWSR1 BA positive & Fibula & - \\
\hline ES-P-09 & P3 & 24 & EWSR1 BA positive & Rib & - \\
\hline ES-P-10 & P2 & 21 & EWSR1 BA positive & Th7 & - \\
\hline ES-P-11 & P2 & 29 & EWS-FLI1 & lliac crest & MD \\
\hline ES-P-12 & P3 & 19 & EWSR1 BA positive & L1 vertebra & MD \\
\hline ES-P-13 & P2 & 31 & EWSR1 BA positive & Humerus & -
\end{tabular}

PN stands for Passage Number; BA stands for Break Apart; PT stands for Primary Tumor; MD stands for Metastasis at diagnosis.

The same approach was used regarding hMSC from healthy donor (HD) samples (Table VI).

BM-hMSC from ES-P $(n=11)$ were provided by LUMC (Leiden University Medical Centre; Leiden, The Netherlands), BM-MSC-HD ( $n=6)$ were provided by the HUSAL (University Hospital of Salamanca; Salamanca, Spain) and BMMSC from patients with benign bone lesion (BM-MSC-BL) $(n=10)$ were provided by the IOR. 
Table VI. Characterization of MSC derived from healthy donors

\begin{tabular}{|c|c|c|}
\hline Sample & PN & Local of extraction \\
\hline BM-DN-TD & $\mathrm{P} 4$ & Iliac crest \\
\hline BM-DN-HB & P5 & Iliac crest \\
\hline BM-DN-001 & P6 & Iliac crest \\
\hline BM-DN-005 & P6 & Iliac crest \\
\hline BM-DN-HU-1 & $\mathrm{P} 4$ & Iliac crest \\
\hline BM-DN-HU-2 & $\mathrm{P} 4$ & Iliac crest \\
\hline BM-DN-TD & $\mathrm{P} 4$ & Iliac crest \\
\hline DP-CM & $\mathrm{P} 5$ & Dental Pulp \\
\hline DP-15 & P6 & Dental Pulp \\
\hline DP-49 & P6 & Dental Pulp \\
\hline COR-3442 & $\mathrm{P} 5$ & Chorion \\
\hline COR-3386 & $\mathrm{P} 5$ & Chorion \\
\hline COR-3412 & P6 & Chorion \\
\hline AM-3481 & P6 & Amnios \\
\hline AM-3386 & P6 & Amnios \\
\hline AM-3396 & P5 & Amnios \\
\hline PLD-2 & $\mathrm{P} 4$ & Placenta \\
\hline PLD-3 & $\mathrm{P} 4$ & Placenta \\
\hline PLD & $\mathrm{P} 5$ & Placenta \\
\hline AD-HADAS & $\mathrm{P} 4$ & Adipose Tissue \\
\hline AD-HADAS-1 & P3 & Adipose Tissue \\
\hline AD-ADIPO3412 & $\mathrm{P} 5$ & Adipose Tissue \\
\hline AD & $\mathrm{P} 4$ & Adipose Tissue \\
\hline
\end{tabular}

PN stands for Passage Number; BM-DN stands for Bone Marrow-Donor; DP stands for Dental Pulp; COR stands for Chorion; AM stands for Amnios; PLD stands for Placenta Donor and AD for Adipose Tissue. 
In addition hMSC derived from different sources were also considered. hMSC derived from adipose-tissue (AD) $(n=4)$, from IOR and HUSAL, placenta derived MSC (PLD) ( $n=3)$ from HUSAL, dental pulp (DP), $(n=3)$ and amniotic membrane (AM) $(n=3)$, chorion membrane (COR) $(n=3)$ from the University of Bologna (Bologna, Italy) were isolated and expanded as previously described [114-119].

Obtainment of hMSC was approved by LUMC, University of Bologna and HUSAL Institutional Review Boards as well as from IOR Ethical Committee

hMSC from low passages, between 1-4, were fully characterized at the immunophenotypic level. All hMSC derived from EWS Patients ( $n=11)$; hMSCHD ( $n=6)$; hMSC derived from adipose tissue $(n=1)$ and hMSC derived from placenta $(n=1)$ are included in this group. hMSC from higher passages $(n=14)$, were only used for CD99 detection further ahead in our study due to the low number of cells.

\subsection{ES tumor samples}

Frozen tissue from ES samples from the Hospital Virgen Del Rocío Tumor Bank, $(n=9)$ were used to assess the expression of CD90, CD105, CD34 and CD45. All H\&E and CD99 stained sections were carefully examined by experienced pathologists, confirming the diagnosis. Diagnosis was confirmed by morphology and CD99 expression. 


\section{DNA-related assays}

\subsection{DNA Extraction}

Cells were scraped from $10 \mathrm{~mm}$ plates in $1 \mathrm{ml}$ of phosphate buffered saline (PBS) in 1,5ml eppendorf, centrifuged at $1500 \mathrm{rpm}$ for $5 \mathrm{~min}$, rewashed with PBS and finally resuspended in $100 \mu$ l of PBS.

Then, DNA extraction was performed with Qiagen DNA mini kit (Qiagen; Hilden, Germany), following manufacturer's instructions. Pellet was then resuspended in $200 \mu \mathrm{l}$ of PBS with $20 \mu \mathrm{l}$ of Proteinase $\mathrm{K}$ and $200 \mathrm{ul}$ of lysis buffer AL. Tubes were incubated at $56^{\circ} \mathrm{C}$ for 10 minutes. The whole volume was added to the extraction columns and these were centrifuged for $1 \mathrm{~min}$ at 8000rpm. Columns were washed with distinct wash buffers, WB1 and WB2 and finally DNA was eluted in $50 \mu \mathrm{l}$ of DEPC water after $5 \mathrm{~min}$ incubation.

Finally, DNA was quantified with NANOdrop (Thermo Scientific, Waltham, MA USA) and the ratios 260/230 and 260/280 were taken in consideration. Ratios below 1.7 were not accepted since they represent contamination by protein, with absorbance at $280 \mathrm{~nm}$, or phenol groups, with absorbance at $230 \mathrm{~nm}$.

\subsection{DNA Laddering Assay}

Cells were treated for different incubation times: 0h, $24 \mathrm{~h}$ and $72 \mathrm{~h}$. After drug incubation, DNA was extracted and quantified with NANOdrop. At least $1 \mu \mathrm{g}$ was loaded in $1 \%$ agarose gels at $100 \mathrm{~V}$ and revealed with Gel Doc 2000 (Bio-Rad; Hercules, CA, United States of America). DNA ladder marker of $1 \mathrm{~Kb}$ (1000pb) was used to further localize DNA fragments (Biotools; Madrid, Spain). 


\subsection{Cell Cycle Analysis}

Propidium iodide (PI) staining was used to analyze cell cycle changes during cell treatment. This molecule has the capacity to intercalate the double helix of the DNA emitting self-fluorescence, which is detectable by Fluorescence-activated cell sorting (FACS) and therefore permits the rateanalysis of cell cycle phases (G0/G1; S-phase; G2/M).

Initially, cells were seeded in 6 well-plates and incubated with the appropriate anti-tumoral agent for $24 \mathrm{~h}$. Then, cells were fixed in $70 \%$ ethanol (Millipore; Billerica, MA, USA) (in PBS 1x) for 30min. After removing the fixing agent through centrifugation, cells were stained with a solution of $0,5 \% \mathrm{PI}$ (1 mg/ml) (Sigma-Aldrich; St. Louis, MO, USA), 0,5\% RNAase (Sigma-Aldrich)in 1x PBS and incubated in the absence of light for $1 \mathrm{~h}$. Later, cells were acquired (20000 events) in a FACSort Cytometer (Becton Dickinson; Franklin Lakes, NJ, USA). Analysis of results was performed using Infinicyt (Cytognos; Salamanca, Spain) demo version, Paint-a-Gate (Becton Dickinson; Franklin Lakes, NJ, USA) and ModFit software (Verity Software House; Topsham, ME, USA).

\subsection{FISH}

ES cell lines and/or hMSC samples, resuspended in culture medium, were centrifuged at $1500 \mathrm{rpm}$ for $5 \mathrm{~min}$ and medium was discharged. Next, cell pellet was homogenized in $200 \mu \mathrm{l}$ of a methanol (Sigma-Aldrich): acetic acid (Merck; Whitehouse Station, NJ, USA) (3:1) solution and was stored at $4^{\circ} \mathrm{C}$. Subsequently, $10 \mu \mathrm{l}$ of cells were fixed by expansion in a slide and dried $10 \mathrm{~min}$ at room temperature (RT).Slides were washed several times in a coplin jar with PBS and finally incubated with pepsin (Sigma-Aldrich) for enzymatic digestion. 
Enzymatic digestion, as a way to permeabilize both plasma membrane and nuclear membrane, was performed, at $37^{\circ} \mathrm{C}$ for $10 \mathrm{~min}$, with a solution of pepsin (Sigma-Aldrich) consisting of $50 \mathrm{ml}$ of $\mathrm{H}_{2} \mathrm{O}$ milliq, $500 \mu \mathrm{l}$ of $1 \mathrm{~N} \mathrm{HCl}$ (Merck) and $250 \mu \mathrm{l}$ pepsin $(10 \mathrm{mg} / \mathrm{ml})$. After that, slides were washed with $2 \mathrm{X}$ saline-sodium citrate (SSC) buffer, at RT for $5 \mathrm{~min}$, incubated with $1 \%$ formaldehyde (Sigma-Aldrich) for 10min, washed once again with 2XSSC for 10 min, dehydrated with ethanol (Millipore) $70 \%, 90 \%$ and $100 \%$ for 5 min each and air dried for $5 \mathrm{~min}$. At same time, probes were prepared in the appropriate buffer at a proportion 1/20 (locus specific probes) or 1/50 (centromeric probes). A volume of $10 \mu \mathrm{l}$ of the diluted probes was applied to the slides. The slides were covered with a glass cover slip and sealed with rubber cement. Using a Hybrite machine (Vysis) denaturation was $75^{\circ} \mathrm{C}$ for 5 min and hybridization was at $37^{\circ} \mathrm{C}$ for at least $16 \mathrm{~h}$. After removing the cover slips post-hybridization washing was done at $46^{\circ} \mathrm{C}$ in $2 \mathrm{XSSC}, 50 \%$ formamide for $5 \mathrm{~min}$ and stained with DAPI (6-diamidino-2-phenylindole) and mounted with Vectashield $\mathrm{H}-1000$ medium (Vector). Digital images were obtained using a Zeiss Axioplan2 epifluorescence microscope (Carl Zeiss Oberkochen, Germany) equipped with a digital camera (ORCA-ER-1394, Hamamatsu Photonics KK, Hamamatsu, Japan). In all cases, one hundred nuclei were counted.

\subsubsection{EWSR1 break-apart probes and EWS-FLI1 fusion probes}

FISH was performed using a commercial EWS break-apart FISH probe (Abbott Laboratories; Abbott Park, IL, USA) for the detection of ES fusion transcripts.

Furthermore, an additional, home-made dual-color FISH probe for the detection of EWSR1-FLI1 rearrangements was developed as follows: three 
BAC (bacteria artificial clones) (CTD-2307I11, RP11-53D4, CTD-2126E12) spanning the chromosomal region (chr11q24.3) and two BAC clones (RP11367E7, RP11-480L23) spanning a region on chromosome (chr22q12.1-12.2) were labeled with spectrum green-dUTP (green signal) (Vysis Inc.; Downers Grove, IL, United States of America) and spectrum red-dUTP, respectively, by nick translation (Vysis Inc.) and purified after adding 10 $\mathrm{\mu g}$ of COT-1 (Invitrogen). Specificity of this probe was determined by hybridizations over metaphases of peripheral blood cells from healthy donors. FISH procedure was performed as described above and the TC71 cell line was used as a positive control of EWSR1-FLI1 rearrangement.

\subsubsection{PARP1 probes}

Two specific and independent PARP1 locus probes (BAC Clones RPII964L17 and RPII-831N20), spanning the chromosomal region 1q41-q42, labeled with Spectrum green-dUTP (green signal) (Vysis) were made.

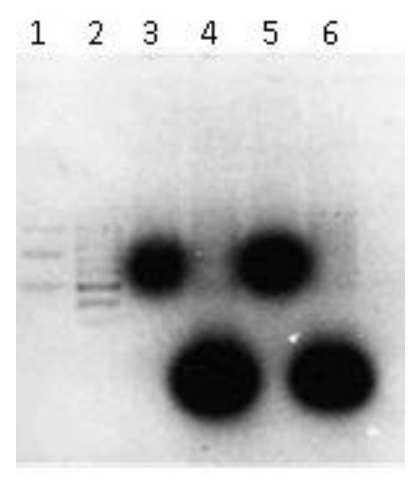

Figure 10. Confirmation on $1 \%$ agarose gel of DNA Probe labeling for RPII964L17: (1 and 2 DNA ladder markers) (3 in red); (4 in green) and positive controls labeled in red (5) and in green (6). 

BACs DNA was extracted and purified using Qiagen Mini/Midi kit (Qiagen).

Then DNAs were labeled with Spectrum green-dUTP (Vysis) by nick translation (Vysis) and purified after adding $10 \mu \mathrm{g}$ of COT-1 (Invitrogen) and confirmed in $1 \%$ agarose gel. A commercially available (CEP 1) probe (Vysis) labeled with spectrum red (red signal) was also used (Figure 10).

To check the specificity of the home-made probes, co-hybridizations using both commercial CEP1 and PARP1 specific probes were performed over metaphases of peripheral blood cells. FISH analysis was done on cell lines fixed on methanol-acetic acid. A volume of $10 \mu \mathrm{l}$ of the diluted probes was applied to the slides. The slide was covered with a glass cover slip and sealed with rubber cement. Using a Hybrite machine (Vysis) denaturation was $75^{\circ} \mathrm{C}$ for 5 min and hybridization was at $37^{\circ} \mathrm{C}$ for at least $16 \mathrm{~h}$. After removing the cover slips posthybridization washing was done at $46^{\circ} \mathrm{C}$ in 2 XSSC, $50 \%$ formamide for 5 min and stained with DAPI (6-diamidino-2-phenylindole) and mounted with Vectashield $\mathrm{H}$ 1000 medium (Vector). Digital images were obtained using a Zeiss Axioplan2 epi-fluorescence microscope (Carl Zeiss Oberkochen, Germany) equipped with a digital camera (ORCA-ER-1394, Hamamatsu Photonics KK, Hamamatsu, Japan). In all cases, one hundred nuclei were counted.

\section{RNA-related assays}

\subsection{RNA extraction}

Around $1 \times 10^{6}$ cells were seeded in $10 \mathrm{~cm}^{2}$ plates. Cells were grown in complete medium for $24 \mathrm{~h}$ and after this time, medium was changed to medium with the specific treatment. After 8 hours and 24 hours, medium was replaced by $1 \mathrm{ml}$ of fresh medium without FBS and antibiotic, and finally the plates were 
scraped. Cell pellet was recovered to $2 \mathrm{ml}$ eppendorf and centrifuged. Medium was carefully removed and cell pellet was resuspended in $1 \mathrm{ml}$ of TRIzol ${ }^{\circledast}$ (Invitrogen). After $5 \mathrm{~min}$ of incubation, the eppendorf was stored at $-80^{\circ} \mathrm{C}$ for eventual precipitation and purification.

For RNA precipitation, all eppendorf were unfrozen at $37^{\circ} \mathrm{C}$ for $10 \mathrm{~min}$

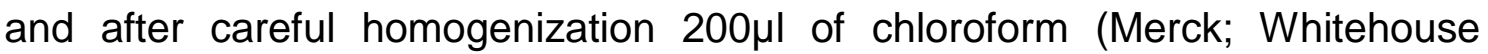
Station, NJ, United States of America) were added to each tube. After 2 minutes of incubation at RT tubes were centrifuged at $4^{\circ} \mathrm{C} 12000 \mathrm{rpm}$ for $15 \mathrm{~min}$. At this point three phases were distinguishable according to the particles that constitute it:

i) upper aqueous phase of RNA,

ii) middle red phase of proteins,

iii) lower white phase of DNA.

The upper-RNA phase was transferred to a new eppendorf and $500 \mu \mathrm{l}$ of 2-propanol (Merck); were added to each tube. Afterwards, tubes were incubated for $10 \mathrm{~min}$ on ice and centrifuged at $4^{\circ} \mathrm{C} 12000 \mathrm{rpm}$ for $10 \mathrm{~min}$. Supernatant was eliminated and $1 \mathrm{ml}$ of sterile ethanol (Sigma-Aldrich) $75 \%$ on DEPC water (Ambion®-LifeTechnologies-Invitrogen; Paisley, UK) was added. After a $5 \mathrm{~min}$ centrifugation, the pellet was resuspended in $50 \mu \mathrm{I}$ DEPC water (Ambion®-Life Technologies-Invitrogen; Paisley, UK).

\subsection{RNA purification}

Purification of RNA was performed with the RNA-extraction Kit from

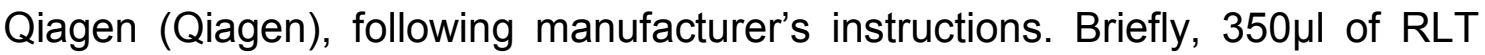
Buffer were added to each tube and after homogenization, $250 \mu$ l of ethanol $100 \%$ (Sigma-Aldrich)) were added. All the volume was passed through a RNA- 
purification column and the complex was centrifuged 10000rpm for 15s at RT. Next, RNA was washed with $350 \mu$ of RW1 buffer and the complex was again centrifuged at RT $10000 \mathrm{rpm}$ for 15s to remove the washing buffer. RDD buffer with $10 \mu$ l DNAase was added over the column in order to eliminate all rests of DNA and following 15min incubation, the complex was again washed with RW1 buffer and centrifuged at RT 10000rpm for 15s. $500 \mu \mathrm{l}$ of RPE buffer were added to the column and after 1 min incubation the complex was centrifuged at RT 10000 rpm for $2 \mathrm{~min}$. This step was performed twice. Finally, RNA was eluted with $50 \mu \mathrm{l}$ of DEPC water, followed by $5 \mathrm{~min}$ incubation at RT and a final centrifugation at RT $1000 \mathrm{rpm}$ for $1 \mathrm{~min}$.

\subsection{RNA Quality Evaluation}

For RNA quantification all samples were measured with the spectrophotometer NANOdrop and the 260nM/280nM and 260/230nM ratios were analyzed. Ideally, both ratios (A260/A280 and 260/230) should be as close to 2.0 as possible. In those cases where ratio was lower than 1.7, the RNA was re-purified since it indicates that the sample is contaminated with proteins or phenol groups, respectively.

Moreover, for RNA evaluation, samples were ran in 1\% agarose gel and the correct RNA presented two clear and separate bands corresponding to and ribosomal units $28 \mathrm{~S}$ and $18 \mathrm{~S}$, where the $28 \mathrm{~S}$ should be slightly more brilliant, as shown in figure 11 . This analysis indicates the stability of the previously extracted RNA. 


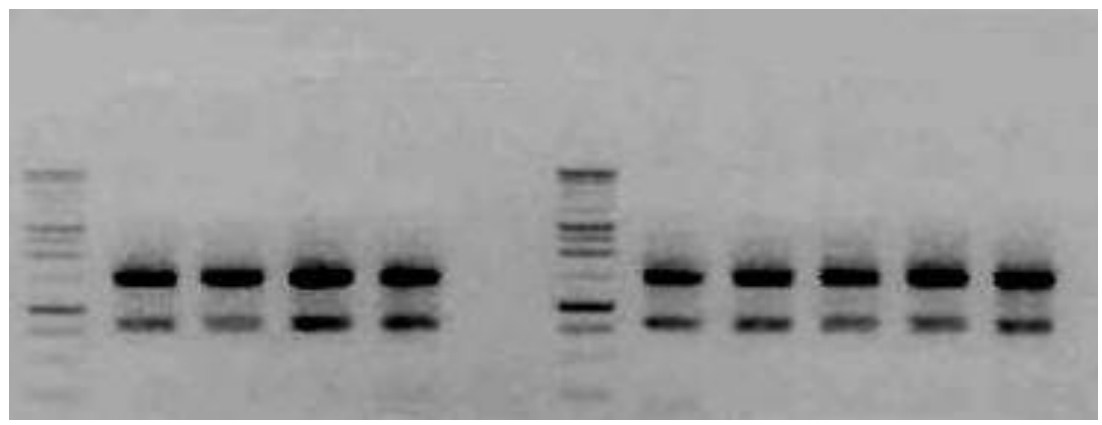

Figure 11. Ribosomal RNA identification, in 1\% agarose gels, two bands are observed: 28s (1500bp) and 18s (750bp). Ladder of $1 \mathrm{~Kb}$.

\subsection{RNA retrotranscription to cDNA}

Retrotranscription from RNA to cDNA was performed, on ice, adding $1 \mu \mathrm{l}$ of OligoDT (18 nucleotides of thymidine) (Thermo Scientific) to $1 \mu$ of RNA and DEPC water (Qiagen) to a total volume of $10 \mu \mathrm{l}$ per sample in $0,2 \mu \mathrm{l}$ eppendorf. After homogenization, samples were denaturized in a thermocycler (Bio-Rad;) at $70^{\circ} \mathrm{C}$ for $10 \mathrm{~min}$ and $4^{\mathrm{a}} \mathrm{C}$ during $2 \mathrm{~min}$. Next, the samples were transferred to ice again and $9 \mu \mathrm{l}$ of the master mix, previously prepared, were added per sample (5X Buffer (Invitrogen,); 0,1M of DTT (Invitrogen), 10nM of DNTP's (Hoffman-La Roche; Basel, Switzerland); $1 \mu$ RNAase inhibitor (Ambion®-Life Technologies-Invitrogen) and $1 \mu \mathrm{l}$ of the retrotranscriptase enzyme (SSII) (Invitrogen). The tubes were correctly homogenized and were relocated in the thermocycler (Bio-Rad;) $50 \mathrm{~min}$ at $42^{\circ} \mathrm{C}$ followed by $15 \mathrm{~min}$ at $70^{\circ} \mathrm{C}$ and finally $10^{\circ} \mathrm{C}$ until the system is turned off. At the end, $30 \mu \mathrm{l}$ of DEPC water (Qiagen) were added to each to complete $50 \mu$ l cDNA final volume. For cDNA correct retrotranscription conformance we performed a "pseudo" q-RT-PCR for the housekeeping gene, glyceraldehyde 3-phosphate dehydrogenase (GAPDH). 


\subsection{Primer design}

Primers were designed using the online platform Primer 3 (http://frodo.wi.mit.edu/ ), according to the sequences obtained in the Ensembl data base for each gene. Basically, the mRNA sequence, present in the "transcription information" was copied to the Primer 3 platform and the primers were chosen according to the following points:

1) Amplicon should be between $90-110 \mathrm{bps}$;

2) Melting temperature TM must be of $60-61^{\circ} \mathrm{C}$.

Primers were tested in a "pseudo" PCR and only those which presented a single amplicon were used for q-RT-PCR (Figure 12).

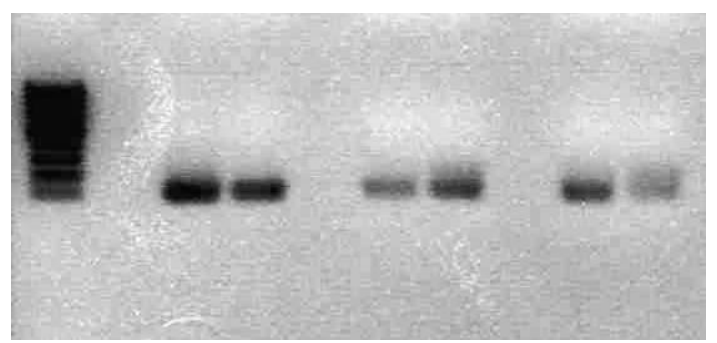

Figure 12. Evaluation of the specificity of the set of primers, in a 1\% agarosegel, to be used in q-RT-PCR. It is possible to observe only one band at around $100 \mathrm{bp}$, determining the specificity of the primers. Ladder of $1 \mathrm{~Kb}$, first row. Rows 2,5 and 8 are empty

\subsection{Quantitative RT-PCR}

cDNA from ES cell lines, wild type and/or treated, were prepared at a concentration of $20 \mathrm{ng} / \mu \mathrm{l}$ and used to perform q-RT-PCR. A master mix was prepared using: $12,5 \mu \mathrm{l}$ of iQ5 SybRGreen (Bio-Rad); $0,4 \mu \mathrm{l}$ of both the forward primer and reverse primer (Table VII); 10,7 $\mu \mathrm{l}$ of DEPC water (Qiagen); $1 \mu \mathrm{g}$ of 
cDNA, to a final volume of $25 \mathrm{ul}$ each well. Consequently, the 96 well plates (Bio-Rad) were sealed and briefly centrifuged at 1300rpm.

Table VII. Primers used for RT-PCR and q-RT-PCR

\begin{tabular}{cc}
\hline Primers Name & Sequence (5'--->3') \\
\hline PARP1_Fw & TCGCTTTTACACCCTGATCCCCCA \\
\hline PARP1_Rv & GCATTTCCACCTTGGCCTGCAC \\
\hline Fusion typel_Fw & GATCCTACAGCCAAGCTCCAAGTCA \\
\hline Fusion typel_Rv & ATAAGAAGGGTTCTGCTGCCCGTAG \\
\hline Fusion typell_Fw & GATCCTACAGCCAAGCTCCAAGTCA \\
\hline Fusion typell_Rv & GATTGGTGGTGTGGGAGGTTGTAT \\
\hline TPT1_Fw & TTGGACTACCGTGAGGATGGTGTG \\
\hline TPT1_Rv & TGTGGATGACAAGCAGAAGCCAGT \\
\hline
\end{tabular}

The reaction was performed in one iQ5 thermocycler (Bio-Rad) and the executed protocol had the following steps:

a) Initial denaturalization - 95은 $\mathrm{min}$;

b) Cyclic reaction $-40 / 50$ cycles of $95^{\circ} \mathrm{C}(15 \mathrm{~s})$ and $59^{\circ} \mathrm{C}(1 \mathrm{~min}), 1$ cycle at $95^{\circ} \mathrm{C}(1 \mathrm{~min})$, and 1 cycle at $65^{\circ} \mathrm{C}(1 \mathrm{~min})$;

c) Melting curve (60 cycles, starting at $65^{\circ} \mathrm{C}$, with a temperature gradient of $+/-0.5^{\circ} \mathrm{C}$ for 10 s each cycle);

d) Hold (indefinitely at $4^{\circ} \mathrm{C}$ ).

The data analysis was performed using the BioRad iQ5 software (BioRad), exporting the CT values, the mean CT (threshold cycle) values and the standard deviations to an excel file. The melting curve was examined to determine if only one clean peak per primer was observed (Figure 13). 

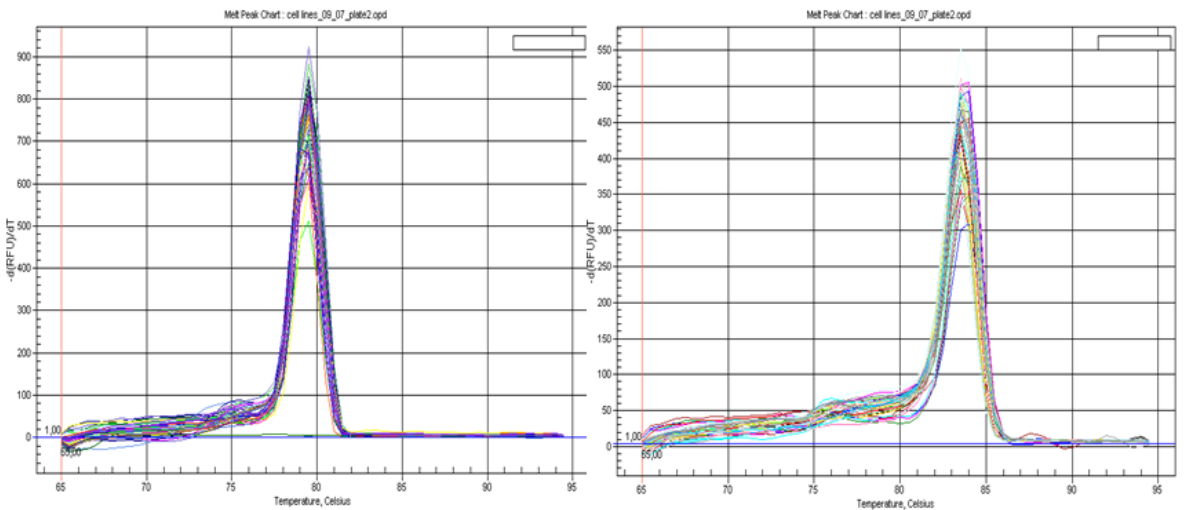

Figure 13. Example of melting peak with a single curve, as obtained by the iQ5 BIORAD software. The TPT1 melting curve is represented on the left (housekeeping gene) and the PARP1 melting curve is represented on the right side.

The CT value of each problem sample was then normalized with the CT value of the housekeeping gene GAPDH, according to the formula established by Pfaffl $\delta^{\wedge} \mathrm{CT}=\mathrm{CT}$ (problem gene)-CT(GAPDH). Finally the formula ratio $=2^{\wedge}(\delta C T)$ was applied and normalized values were then represented in a graphic. Each set of primers were separately tested for their efficiency in a standard curve with dilution of WT cDNA at $20 \mathrm{ng} / \mu \mathrm{l}, 10 \mathrm{ng} / \mu \mathrm{l}, 1 \mathrm{ng} / \mu \mathrm{l}$ and $0,1 \mathrm{ng} / \mu \mathrm{l}$. CT values were used to determine the slope of each standard curve and the efficiency was calculated using the following formula Efficiency $=10^{\wedge}(-$ 1/slope) being, only the values between 1.5-2 accepted.

\subsection{Low density microarray}

\subsubsection{RNA extraction}

TC71 cell line was seeded and when $60 \%$ confluence was reached, the cells were treated with the appropriate concentrations of the specific treatment. After $18 \mathrm{~h}$ treatment, the medium was discharged and cells were scraped in $1 \mathrm{ml}$ of medium without FBS and collected to a $2 \mathrm{ml}$ eppendorf. RNA purification was 
performed with the RNA mini Qiagen kit (Qiagen), always keeping in mind that DEPC water couldn't be used and that DNA on-column digestion was essential. After extraction, $0.5 \mu \mathrm{g}$ of RNA was again digested with $2 \mu$ of DNA elimination buffer, GE for $5 \mathrm{~min}$ at $42^{\circ} \mathrm{C}$. RNA was measured in the NANOdrop and only samples with $260 / 280$ ratio $>2$ and $260 / 280$ ratio $>2$ were used.

\subsection{2. cDNA synthesis}

cDNA synthesis was performed using the RT2 First strand kit (Qiagen), following manufacturer's instructions. Briefly, a mix containing 5xBuffer P3, Control P2, RE3 Reverse Transcriptase Mix and RNAase-free-water was prepared and $10 \mu \mathrm{l}$ of this mix were added to the RNA solution (containing DNA elimination buffer from the previous step). Tubes were incubated for $42^{\circ} \mathrm{C}$ for exactly $15 \mathrm{~min}$ and the reaction was stopped at $95^{\circ} \mathrm{C}$ for $5 \mathrm{~min}$. After this step, $91 \mu$ of RNAase-free-water were added to each reaction.

\subsubsection{Quantitative-RT-PCR for Low density microarray}

A PCR mix was prepared using $1350 \mu$ of $2 \mathrm{X} \mathrm{RT}^{2}$ SYBR Green Mastermix, $102 \mu$ of the cDNA synthesis product and $1248 \mu$ l of RNAase-freewater (all from Qiagen). The total volume of $2700 \mu$ l was transferred to a reservoir and using a multi-dispenser pipet, $25 \mu \mathrm{l}$ were added per well. Plates were carefully sealed, centrifuged for $1 \mathrm{~min}$ at $3300 \mathrm{rpm}$ at $\mathrm{RT}$ to remove bubbles and placed on ice. The following protocol was set in an iQ5 BioRad thermo cycler (Bio-Rad): $10 \mathrm{~min}$ cycle at $95^{\circ} \mathrm{C}$ for Taq Polymerase activation, followed by 40 cycles of $15 \mathrm{~s}$ at $95^{\circ} \mathrm{C}$ and $1 \mathrm{~min}$ at $60^{\circ} \mathrm{C}$ to perform fluorescence data collection (Table VIII). 
Table VIII. Description of the genes included in PCR array.

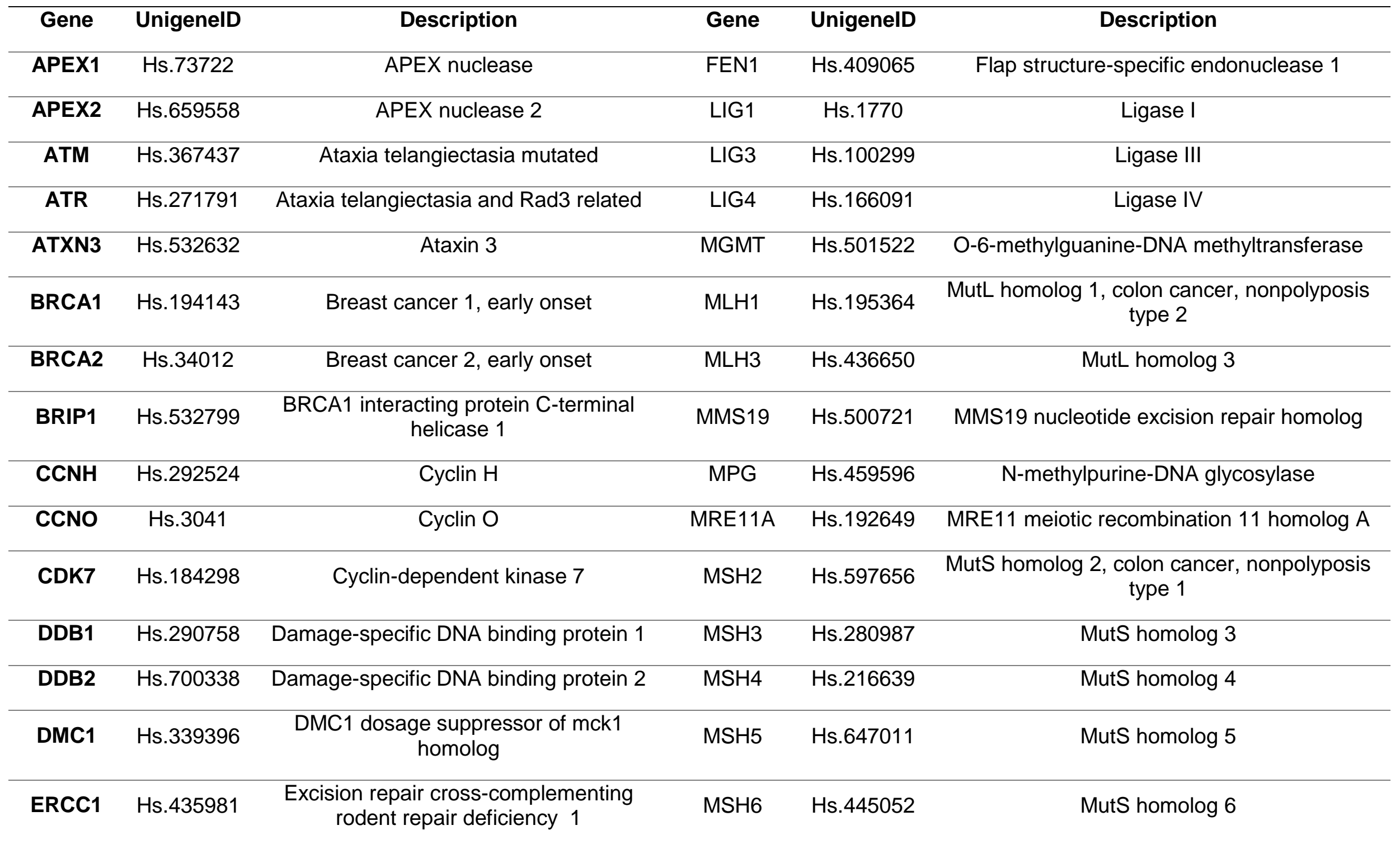




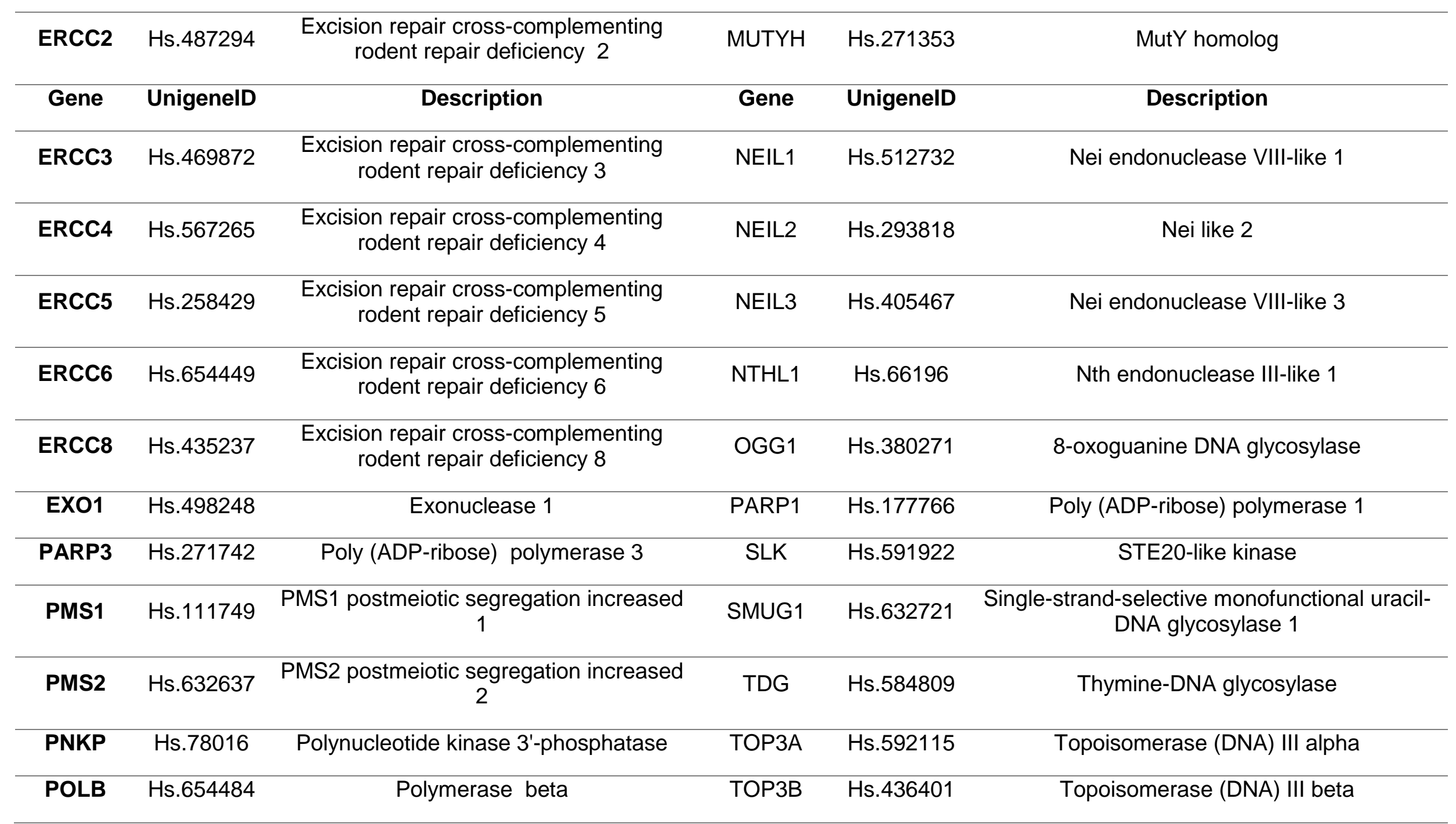




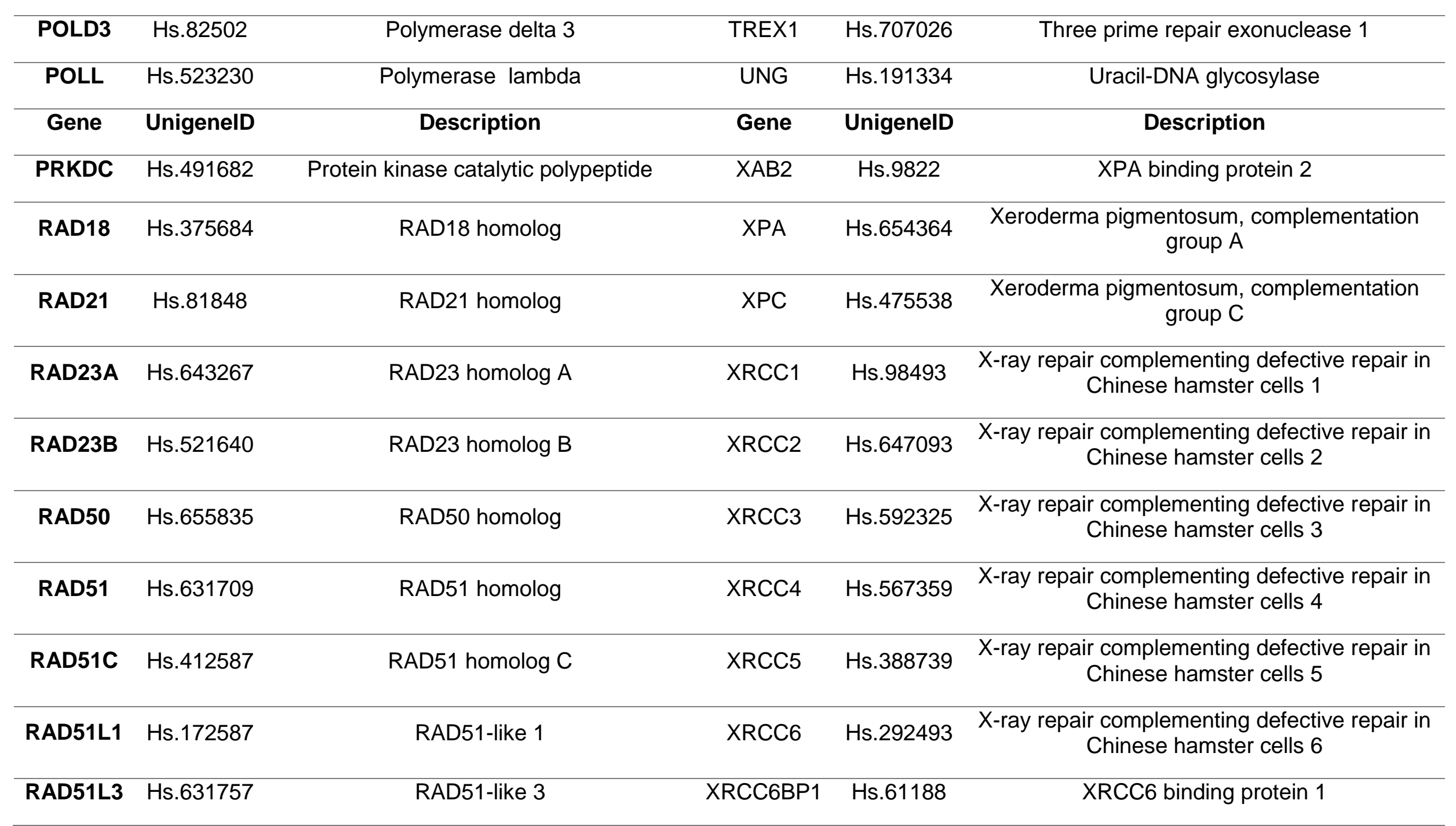




\begin{tabular}{cccccc}
\hline RAD52 & Hs.709202 & RAD52 homolog & RPA1 & Hs.461925 & Replication protein A1 \\
\hline RAD54L & Hs.642042 & RAD54-like & RPA3 & Hs.487540 & Replication protein A3 \\
\hline RFC1 & Hs.507475 & Replication factor C (activator 1) 1 & RPLP0 & Hs.546285 & Ribosomal protein, large
\end{tabular}

PCR array includes internal controls: Rows $A$ to $G$ correspond to genes involved in either DNA damage or DNA repair pathways, whereas Row H corresponds to housekeeping genes: ACTB, $\beta$-actin; B2M, beta-2-microglobulin, GADPDH, glyceraldehydes-3phosphate dehydrogenase and HPRT1, hypoxanthine phosphorybosil transferase-1 and the following internal controls, HGDG, Human genomic DNA contamination; PPC, positive controls and RTC, reverse transcription control. 


\subsubsection{Quality Control}

The CT values were collected as described earlier using the iQ5 software, and were exported to an Excel file. Data analysis was performed using the $\delta \delta C T$ method. All CT values reported as greater than 35 or as N/A (not amplified) were changed to 35 and assumed as a negative call.

The genomic DNA controls (GDC) were subsequently evaluated and when CT for Genomic DNA was greater than 35 we considered that there was no genomic contamination.

Evaluation of the Reverse Transcription Control (RTC) and Positive PCR control (PPC) was performed using the following formula: $\delta C T=A$ verage $C T^{R T C} \_$ Average $\mathrm{CT}^{\mathrm{PPC}}$. Only values lower than 5.0 were accepted, since higher values indicate contamination with salts which can inhibit the PCR reaction leading to false negatives. Finally, only values of PPC (average per plate) of $20 \pm 2$ were accepted.

\subsubsection{Analysis of genes of Interest}

Global analysis of the PCR array was performed through the online software http://geneweb.sabiosciences.com/gncpro/gncpro.php.

Here, quality control was again revised as well as distribution of the population. Single gene analysis was observed and group plotting was performed using the internal methods of quantification provided by this software. Cluster analysis was performed according to software instructions. 


\section{Protein-related assays}

\subsection{Protein extraction}

After the appropriated incubation time, cells were scraped in $1 \mathrm{ml}$ of cold $1 \mathrm{x}$ PBS and the volume was transferred to an eppendorf. After centrifugation, PBS was removed and the pellet was resuspended with $60 \mu$ l of lysis buffer, and incubated for $5 \mathrm{~min}$ on ice. Finally tubes were centrifuged for $15 \mathrm{~min}$ at $13000 \mathrm{rpm}$ $\left(4^{\circ} \mathrm{C}\right)$ and the pellet was removed.

Lysis buffer was prepared in milliQ $\mathrm{H}_{2} \mathrm{O}$ complemented with $10 \mathrm{mM}$ of Tris$\mathrm{HCl} \mathrm{pH=7.5;100mM} \mathrm{NaCl;} \mathrm{1mM} \mathrm{of} \mathrm{EDTA} \mathrm{(Sigma-Aldrich);} \mathrm{1 \%} \mathrm{of} \mathrm{NP-40} \mathrm{(Sigma-}$ Aldrich); $0.5 \%$ of sodium desoxicolate (Sigma-Aldrich) and $0,1 \%$ SDS, to a total volume of $100 \mathrm{ml}$. At the time of the extraction $950 \mu \mathrm{l}$ of lysis buffer were complemented with phosphatases inhibitors: $5 \mu \mathrm{l}$ of $1 \mathrm{mM} \mathrm{NaF}$ (Sigma); $5 \mu \mathrm{l}$ of $1 \mathrm{mM} \mathrm{NaOv}$ (Sigma) and $40 \mu \mathrm{l}$ of protease inhibitor (Hoffman-La Roche; Basel, Switzerland). Final solution was permanently kept on ice.

\subsection{Protein Quantification: the Bradford method}

Protein extracts were quantified using the Bradford method. The Bradford method for protein quantification is a colorimetric assay able to determine the amount of protein in each sample through the absorbance of the established Bradford reagent-protein complexes. The Bradford reagent alone is red and turns to blue when specific and relatively stable complexes are established with protein. Higher protein concentrations lead to stronger shades of blue.

Five standards were prepared by diluting BSA $2 \mu \mathrm{g} / \mu \mathrm{l}$ in milliQ $\mathrm{H}_{2} \mathrm{O}$ (Table IX). Samples were prepared by adding 1 to $4 \mu$ of the extract to the appropriate amount of milliQ $\mathrm{H}_{2} \mathrm{O}$ and $200 \mu \mathrm{l}$ of Bradford reagent, up to a total of $1 \mathrm{ml}$. After 
vortex, absorbance was read in a spectrophotometer (Bio-Rad) at 595nm. Protein concentration was determined according to the Beer-Lambert Law.

Table IX. Standards for protein quantification by the Bradford method

\begin{tabular}{|c|c|c|c|}
\hline Standard $(\mu \mathrm{g} / \mu \mathrm{l})$ & BSA $(2 \mu \mathrm{g} / \mu \mathrm{l})$ & $\mathrm{H}_{2} \mathrm{O}$ & Bradford \\
\hline 0 & $0 \mu \mathrm{l}$ & $800 \mu \mathrm{l}$ & $200 \mu \mathrm{l}$ \\
\hline 2 & $1 \mu \mathrm{l}$ & $799 \mu \mathrm{l}$ & $200 \mu \mathrm{l}$ \\
\hline 4 & $2 \mu \mathrm{l}$ & $798 \mu \mathrm{l}$ & $200 \mu \mathrm{l}$ \\
\hline 6 & $3 \mu l$ & $797 \mu \mathrm{l}$ & $200 \mu \mathrm{l}$ \\
\hline 8 & $4 \mu \mathrm{l}$ & $796 \mu \mathrm{l}$ & $200 \mu \mathrm{l}$ \\
\hline 10 & $5 \mu \mathrm{l}$ & $795 \mu \mathrm{l}$ & $200 \mu \mathrm{l}$ \\
\hline
\end{tabular}

\subsection{Electrophoresis and Transference}

Stacking gels were prepared at $4 \%$, with $12,68 \mathrm{ml}$ milliQ $\mathrm{H}_{2} \mathrm{O} ; 2 \mathrm{ml}$ acrylamide (Bio-Rad); $5 \mathrm{ml}$ of Tris $\mathrm{HCl}(0,5 \mathrm{M}, \mathrm{pH} 6.8) ; 200 \mu \mathrm{l}$ of $10 \%$ SDS; $20 \mu \mathrm{l}$ of TEMED (Sigma-Aldrich;) and 100 $\mu \mathrm{l}$ of ammonium-persulphate (APS) (SigmaAldrich) $10 \mathrm{mg} / \mathrm{ml}$.

For immunoblotting three different resolving gels were used according to the size of the target protein. For smaller proteins (10-37 KD) resolving gels at $12 \%$ acrylamide; for medium size proteins (37-100KD) gels at $10 \%$ acrylamide and finally for larger proteins (>100KD) gels at $8 \%$ acrylamide. Resolving gels were prepared at $12 \%$, with $8,68 \mathrm{ml}$ of milliQ $\mathrm{H}_{2} \mathrm{O} ; 6 \mathrm{ml}$ of acrylamide (Bio-Rad); $5 \mathrm{ml}$ of Tris- $\mathrm{HCl}(1,5 \mathrm{M}, \mathrm{pH} 8.8) ; 200 \mu \mathrm{l} 10 \%$ SDS (Sigma-Aldrich) and the polymerizing agents $20 \mu \mathrm{l}$ TEMED (Sigma-Aldrich) and $100 \mu \mathrm{l}$ APS $100 \mathrm{mg} / \mathrm{ml}$ (Sigma-Aldrich). 
Resolving gels at $10 \%$ were prepared with $9,68 \mathrm{ml}$ of milliQ $\mathrm{H}_{2} \mathrm{O} ; 5 \mathrm{ml}$ of acrylamide (Bio-Rad; Hercules, CA, United States of America); $5 \mathrm{ml}$ of Tris-HCl $(1,5 \mathrm{M}, \mathrm{pH} 8,8) ; 200 \mathrm{ul} 10 \%$ SDS (Sigma-Aldrich) and the polymerizing agents $20 \mu \mathrm{l}$ TEMED (Sigma-Aldrich;) and $100 \mu \mathrm{l}$ APS 100mg/ml (Sigma-Aldrich). $8 \%$ resolving gels were prepared with $11,68 \mathrm{ml}$ of milliQ $\mathrm{H}_{2} \mathrm{O} ; 4 \mathrm{ml}$ of acrylamide (BioRad); $5 \mathrm{ml}$ of lower Tris-HCl (1,5M, pH8.8); $200 \mu \mathrm{l} 10 \%$ SDS (Sigma-Aldrich) and the polymerizing agents $20 \mu \mathrm{l}$ TEMED (Sigma-Aldrich;) and $100 \mu \mathrm{l}$ APS $100 \mathrm{mg} / \mathrm{ml}$ (Sigma-Aldrich). Loading buffer $4 \mathrm{X}$ was prepared with Tris $\mathrm{HCl} \mathrm{pH}=6.8$ 200nM; 40\% glycerol (Sigma-Aldrich); 4\% SDS (Sigma-Aldrich); 0,05\% bromophenolblue (Sigma-Aldrich) milliQ $\mathrm{H}_{2} \mathrm{O}$ to $100 \mathrm{ml}$ and finally $4 \%$ of $\beta$-mercapto-ethanol (Sigma-Aldrich). Loading Buffer $4 \mathrm{x}$ was added to $50 \mu \mathrm{g}$ of protein extract and the final solution was denaturalized (95으 for $5 \mathrm{~min})$.

After polymerization, the samples, with the loading buffer previously denaturalized, were loaded in the acrylamide gel, and electrophoresis was ran at 40V-90V until reaching the resolving gel and since them at $120 \mathrm{~V}$ for the appropriate time (time differs depending on the acrylamide \%. After electrophoresis, the proteins were transfered to PVDF Immobilon-FL membranes (Millipore). In short, membranes were activated for $2 \mathrm{~min}$ in methanol (SigmaAldrich), equilibrated 2 min in milliQ $\mathrm{H}_{2} \mathrm{O}$ and left in $10 \%-20 \%$ transference buffer (100ml transfer buffer 10X; 700ml milliQ $\mathrm{H}_{2} \mathrm{O}$ and $100 \mu \mathrm{l}-200 \mu \mathrm{l}$ methanol (SigmaAldrich). In the western blot (WB) cassettes, reagents were placed in the following order: sponge; four sheets of Whatman paper; PVDF membrane; acrylamide gel; four sheets of Whatman paper and sponge. Transference ran for $4 \mathrm{~h}$ at $40 \mathrm{~V}$ at $4^{\circ} \mathrm{C}$. 
After transference, PVDF membranes were incubated for 2-3 minutes with red Ponceau $(0,5 \mathrm{~g}$ of red Ponceau in $1000 \mathrm{ml}$ of acetic acid (Merck) to determine if transference was perfectly achieved. Membranes were washed several times in TBS-T (40ml of TBS; $20 \mathrm{ml}$ of Tween 20 (Merck) and milliQ $\mathrm{H}_{2} \mathrm{O}$ for a total volume of $2000 \mathrm{ml}$ with $0.5 \%$ BSA (Sigma-Aldrich).

4.4 Blocking, incubation with primary and secondary antibodies and western blotting developing

Membranes were left blocking for unspecific binding with a solution of $5 \%$ BSA (Sigma-Aldrich) in TBS-T for $1 \mathrm{~h}$. After membrane blockage, membranes were incubated with the primary antibody at $4^{\circ} \mathrm{C}$ overnight (Table $\mathrm{X}$ ).

Table X. Primary antibodies for WB

\begin{tabular}{ccccc}
\hline Primary Antibody & MW (KD) & Host species & Dilution & Commercial \\
\hline MAb anti-BRCA2 & 460 & Mouse & $1: 500$ & Calbiochem\#OP95 \\
\hline Anti-53BP1 & 450 & Rabbit & $1: 1000$ & Cell Signaling\#4937 \\
\hline MAb anti-BRCA2 & 380 & Rabbit & $1: 500$ & Abcam\#ab2957 \\
\hline Anti 53 BP1 & 220 & Rabbit & $1: 500$ & Abcam\#ab21083 \\
\hline MAb anti-XPG & 133 & Mouse & $1: 1250$ & Abcam\#ab46 \\
\hline Anti-PARP & $24,89,116$ & Rabbit & $1: 1000$ & Cell Signaling\#9542 \\
\hline Anti-RAD52 & 46 & Rabbit & $1: 500$ & Abcam\#ab129563 \\
\hline MAb anti-Rad51 & 37 & Mouse & $1: 500$ & Abcam\#ab213 \\
\hline Anti-ERCC1 & $33-36$ & Mouse & $1: 500$ & Abcam\#2356 \\
\hline cleaved-caspase-3 & 17,19 & Mouse & $1: 1000$ & Cell Signaling\#9661 \\
\hline Anti-actin-N & 42 & Rabbit & $1: 1000$ & Sigma\#A2103 \\
\hline Anti-calnexin & 90 & Rabbit & $1: 500$ & Santa Cruz \#sc-11397 \\
\hline
\end{tabular}


After that, membranes were washed with TBS-T twice for $10 \mathrm{~min}$. Secondary antibody was incubated for $1 \mathrm{~h} \mathrm{RT.}$

Dy64 conjugated secondary antibodies (Thermo Fischer Scientific; Waltham, MA, United States of America), anti-mouse-red and anti-rabbit-green (1:10000) in TBS-T 0.5\% BSA (Sigma-Aldrich) were incubated for one hour at RT. After washing the membranes twice for $5 \mathrm{~min}$ with TBS-T, the membranes were developed by fluorescence in Odyssey (Li-Cor Biosciences; Lincoln, NE, USA) version 7.0.

\subsection{Flow Cytometry}

\subsubsection{Caspase-3 detection assay}

Activity of caspases 3 and 7 was determined by a luminescence assay Caspase Glo (Promega; Madison, WI, USA). Initially, cells were seeded in white walled 96 well plates for $24 \mathrm{~h}$ and then treated with the drug for $24 \mathrm{~h}$. After incubation, a mixture of Caspase Glo buffer and substrate, freshly prepared at RT, was added, $100 \mu \mathrm{l}$ per well. Plates were incubated for $1 \mathrm{~h}$ and the luminescence was read in an Infinite ${ }^{\circledR}$ F500 tecan plate reader (Tecan; Maennedorf, Switzerland). Caspases 3 and 7 activity was calculated subtracting the blank value (well with medium but no cells) and normalized with the control (untreated cells).

\subsubsection{Multiparameter Flow Cytometry}

Cells were collected as described earlier, and $2 \times 10^{5}$ cells were used for each tube. Cells were centrifuged and washed once with cold PBS. Multiparametric flow cytometry (MFC) immunophenotypic studies were performed 
using the following monoclonal antibodies (MAb) combinations: (AF700/AmCyan//PerCPCy5.5/PacificBlue/PE/FITC/APC/PerCPCy7):CD90/CD4 5/CD34/CD105/CD99, CD166, CD271/CD54,CD106,CD19/CD117,CD73,HLADR/CD144,CD13,andCD10. Controls were performed using a tube using fluorescence minus one, only with the common antibodies: CD105; CD90; CD45 and CD34 (Table XI).

Table XI. Panel of antibodies and Fluorescence used for MFC

\begin{tabular}{ccc}
\hline Antibody & Dilution & Commercial \\
\hline CD90-AF700 & $1: 100$ & Biolegend \\
\hline CD45-AmCyan & $4: 100$ & Becton Dickinson, BioSciences \\
\hline CD34-PerCP & $1: 100$ & Becton Dickinson \\
\hline CD105-PB & $4: 100$ & Exbio \\
\hline CD99-PE & $1: 100$ & BD Pharmingen \\
\hline CD166-PE & $1: 100$ & Beckman Coulter \\
\hline CD271-PE & $1: 100$ & Becton Dickinson \\
\hline CD54-FITC & $1: 100$ & ED Bio \\
\hline CD106-FITC & $1: 100$ & Becton Dickinson \\
\hline CD19-FITC & $1: 100$ & Becton Dickinson \\
\hline CD117-APC & $3: 100$ & eBioscience \\
\hline CD73-APC & $3: 100$ & Becton Dickinson \\
\hline HLA DR-APC & $3: 100$ & Becton Dickinson \\
\hline CD14-PeCy7 & $5: 100$ & Becton Dickinson \\
\hline CD13-PeCy7 & $5: 100$ & Becton Dickinson \\
\hline CD10-PeCy7 & $5: 100$ & \\
\hline
\end{tabular}

Cells were stained out of light for $15 \mathrm{~min}$ with the appropriate conjugation of MAb. For CD99 staining, functional studies were performed by indirect immunofluorescence using clone 013 (Signet; Dedham, MA, USA), as primary 
antibody diluted 1:80 and goat anti-mouse FITC (Pierce Biotechnology; Rockford, IL, USA), diluted $1: 100$, as secondary antibody. No less than $2 \times 10^{5}$ cells were acquired.

\subsection{Immunofluorescence}

Around 20000 cells were cultured in $0.1 \%$ gelatin (Sigma-Aldrich) coated covered slides placed in 24 -well pates for $24 \mathrm{~h}$. Cells were incubated with the respective drug for $8 \mathrm{~h}$ or $24 \mathrm{~h}$. Combination between the fixative agents: methanol (Sigma-Aldrich), paraformaldehyde (Sigma-Aldrich) and permeabilization agents: acetone (Merck); TritonX-100 (Sigma-Aldrich) were tested for each primary antibody (Figure 14).

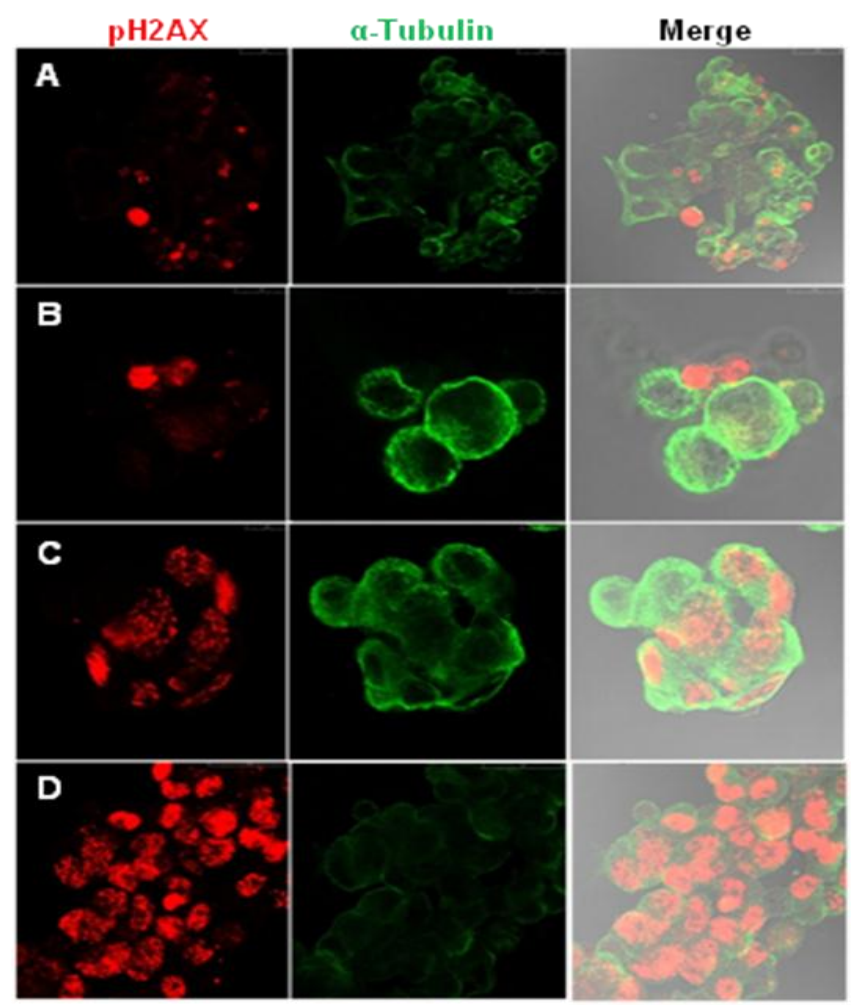

Figure 14. Distinct combinations of agents to fix and permeabilize cells. A, combination of Paraformaldehyde and Methanol; $B$, combination of paraformaldehyde and Triton x-100 0,1\%; C, combination of Methanol and Acetone; D, combination of Methanol and Triton $x-1000,1 \%$. Cells were treated with high doses of Trabectedin for 24 hours and combination D was chosen. 
After drug incubation, cells were fixed for $15 \mathrm{~min}$, permeabilized for $30 \mathrm{~min}$, washed with PBS and blocked with PBS 2\% BSA (Sigma-Aldrich) for 30min. Primary antibody (Table XII), was incubated overnight at $4^{\circ} \mathrm{C}$, and secondary antibody, goat anti-rabbit Cy3/ goat anti-Mouse-Alexa Fluor488/ goat anti-Rabbit Cy2 (Jackson ImmunoResearch Laboratories, Inc.; West Grove, PA, USA) was incubated for 1h. Cells were washed three times and nuclear DNA was counterstained with DAPI (Vector Labs). Cells were mounted using Vectashield H-1000 medium (Vector Labs). A Leica inverted fluorescence microscope (Leica Microsystems; Wetzlar, Germany) connected to a digital video camera (Leica DC100) was used to obtain confocal or fluorescence images. Confocal image analysis was performed using the LSM 5 Image Browser (program version 2.8).

Alternatively, an assay with $6 \mathrm{~h}$ drug incubation followed by washout for $16 \mathrm{~h}$ was also performed. Here, cells were treated in medium with drug for $6 \mathrm{~h}$, and then medium was changed to drug-free medium and incubated for more $16 \mathrm{~h}$. After this, fixation was performed as described above and the global protocol was developed.

Table XII. Primary Antibodies used for Immunofluorescence

Primary Antibody Host species Dilution Commercial

\begin{tabular}{cccc}
\hline Mab anti-pH $\mathbf{H}_{\mathbf{2}}$ Ax & $\mathrm{Rb}$ & $1: 100$ & Cell Signaling \#9718 \\
\hline Anti-53 BP1 & $\mathrm{Rb}$ & $1: 200$ & Abcam \#ab21083 \\
\hline Anti-53 BP1 & Ms & $1: 100$ & Cell Signaling \#4937 \\
\hline Anti-alpha tubulin & Ms & $1: 100$ & Calbiochem \\
\hline
\end{tabular}




\section{Invasion and migration assays}

\subsection{Migration assays}

Invasion assays were performed by wound healing, where 50000 cells were seeded in 24-well plates and when confluence reached $90 \%$, a wound was performed with a pipette tip from one side to the other. Medium with drug was added and incubated for $8 \mathrm{~h} / 24 \mathrm{~h} / 48 \mathrm{~h}$ and/or $72 \mathrm{~h}$. After drug exposure, cells were washed, stained with $1 \%$ crystal violet (Sigma-Aldrich) in $2 \%$ ethanol (SigmaAldrich) and observed with a Leica Microscope (Leica Microsystems; Wetzlar, Germany), where pictures were obtained.

\subsection{Invasion assays}

Migration assays are based on the evidence that invasive cells produce matrix metalloproteases that destroy the endothelial cells and allow tumour cells to invade peripheral tissue thus favouring tumour growth and metastasis. Here, we used Millipore invasion kit assay (Millipore), and followed manufacturer's instructions (Figure 15). Therefore, 300000 cells in the appropriate drug concentration were seeded on the top chamber in medium without serum (starvation), and medium with serum was added to the bottom chamber, as a chemo-attractive. After $72 \mathrm{~h}$ of incubation the non-invading cells were removed from inside the inserts with sterile cotton-buds. The staining solution was added on the bottom chamber at incubated for 20 min. After, inserts were washed in $\mathrm{H}_{2} \mathrm{O}$ to remove the excess of the staining solution and were left drying at RT for some hours. Photos were captured in an Optical biological loupe (Olympus Corporation; Tokyo, Japan). 


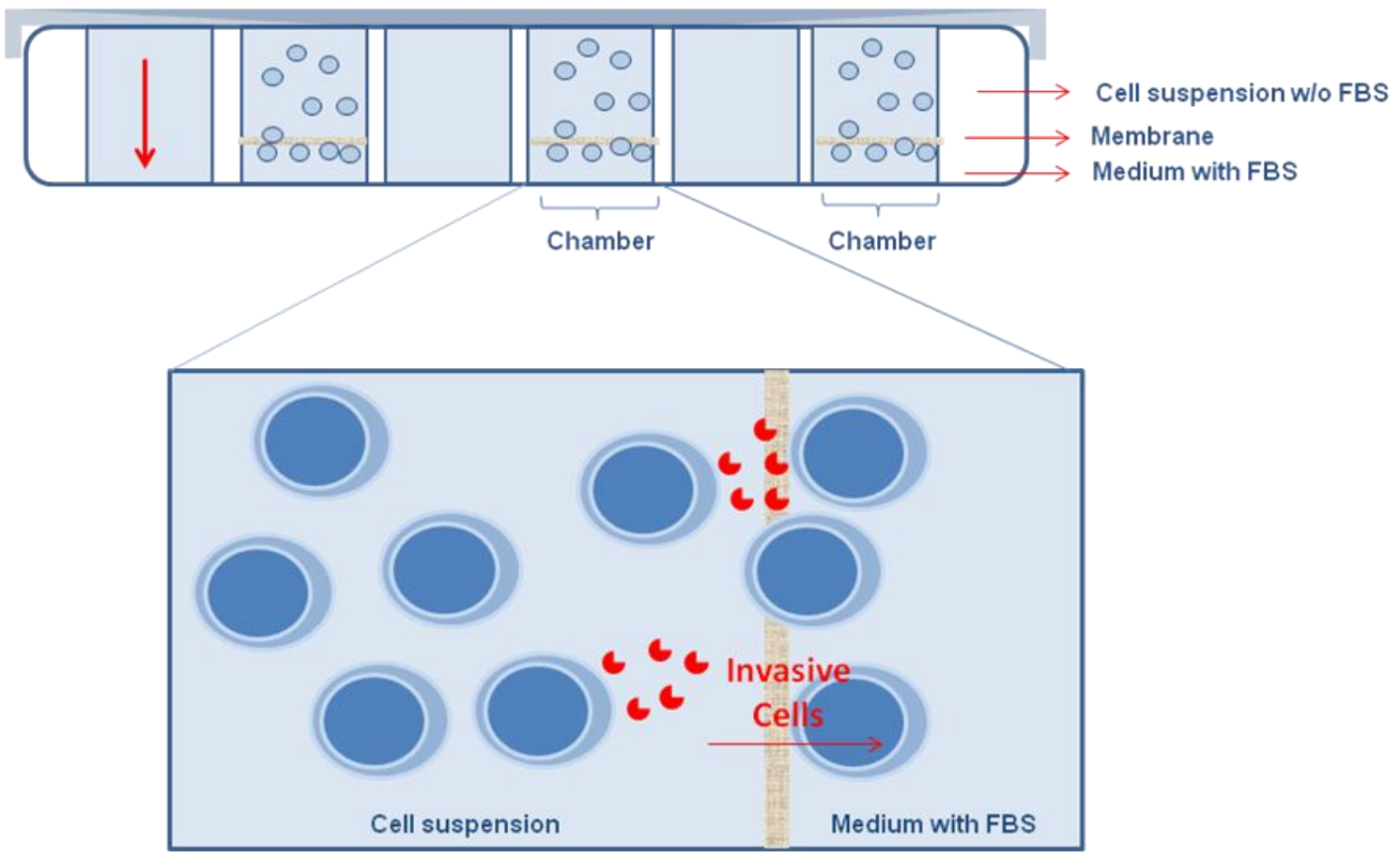

Figure 15. Invasion Assays schematic system

\section{Cell Proliferation assays}

The rates of proliferating cells were measured by the absorbance emitted by a complex formed after the reduction of tetrazolium to formazan, by the mitochondrial dehydrogenase present in proliferating-live cells. Proliferation assays were determined in all cell lines treated with the respective drug for $72 \mathrm{~h}$, by MTT assay.

Briefly, cell were seeded on 24-well culture plates or 96-well culture plates and incubated for $72 \mathrm{~h}$. Medium was then removed and cells were incubated for 1h with a 1:10 MTT solution (Sigma-Aldrich). Medium was later removed and replaced by $100-500 \mu l$ of DMSO (Merck). Absorbance was later read in an Infinite ${ }^{\circledR}$ F500 tecan plate reader (Tecan) at 570nm. The number of cells seeded for each assay was depending on the cell line type and growth rate. Cells were counted by trypan blue and seeded $24 \mathrm{~h}$ prior treatment in complete medium. 
Alternatively, a different cell counting kit was used (Sigma Counting Kit) (Sigma-Aldrich). Cells were seeded in 96-well plates and grown for $24 \mathrm{~h}$ in medium with FBS. Cells were treated in medium with drug for $72 \mathrm{~h}$. After incubation, $10 \mu \mathrm{l}$ of CCK-8 reagent at $37^{\circ} \mathrm{C}$ was added to each well, and plates were incubated for $1-4 \mathrm{~h}$ at $37^{\circ} \mathrm{C}$. Absorbance was measured at $450 \mathrm{~nm}$ using an Infinite ${ }^{\circledR}$ F500 tecan plate reader (Tecan).

This assay proved to be more sensitive and practical than the MTT assay. Here, a solution containing WST-8 (complex similar to MTT) produces a formazan dye, when reduced by dehydrogenases present in cells generating a formazan product able to be detected. The amount of formazan dye formed is proportional to the number of living cells.

\section{Differentiation assays}

\subsection{Osteogenic Differentiation}

Osteogenic differentiation of MSC is induced when a monolayer cell culture is growing in a pro-osteogenic cocktail. The standard conditions for this differentiation include: dexamethasone, ascorbic acid-2-phosphate and betaglycerophosphate. Mineralized deposits can appear after a week, nevertheless treatment is often maintained for up to three weeks in order to maximize the number and size of mineralizing nodules. Addition of calcium may also be used in order to increase in vitro mineralization. Early signs of osteogenesis include an increase in bone-specific alkaline phosphatase activity. At the end of the treatment, cells are fixed and stained with Alizarin-Red S solution (SigmaAldrich). 
Herein, a minimum of $5 \times 10^{4}$ hMSC were cultured on glass slides for two days in cell culture medium. Afterwards, cells were cultured for 21 additional days in the appropriate differentiation medium. Briefly, regarding osteogenic differentiation, cells were cultured in Alpha-MEM (Gibco ${ }^{\circledR}$ ) supplemented with 10\% FBS $\left(\right.$ Gibco $\left.^{\circledR}\right) ; 1 \%$ P/S (Gibco $\left.{ }^{\circledR}\right) ; 0,1 \mu \mathrm{M}$ dexamethasone (Sigma-Aldrich);

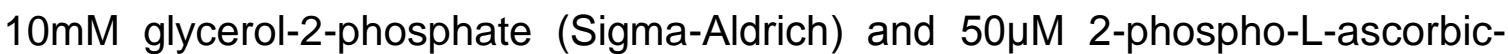
acid (Sigma-Aldrich) and later on, stained for alkaline phosphatase activity detected with BT-BCIP (Hoffman-La Roche; Basel, Switzerland) and calcium deposits with alizarin red (Sigma-Aldrich).

\subsection{Adipogenic Differentiation}

Formation of mature adipocytes occurs following treatment of MSC with a medium supplemented with dexamethasone (Sigma), isobutyl-methylxanthine, insulin (Sigma), and PPARgamma agonist (Sigma) The medium should be changed twice a week for a period of three weeks. Alternatively, MSC can be exposed to three cycles of a treatment which alternates three days of culture in induction medium followed by two days in maintenance medium. The induction medium contains indomethacin instead of PPAR gamma agonist, and the maintenance medium contains insulin. The appearance of adipocytes containing lipid-filled droplets can be assessed by staining with Oil-red $O$.

Adipogenic differentiation was evaluated after incubation in Alpha-MEM $\left(\right.$ Gibco $\left.^{\circledR}\right)$ medium supplemented with $10 \%$ FBS (Gibco $\left.{ }^{\circledR}\right) ; 0,1 \mu \mathrm{M}$ dexamethasone (Sigma-Aldrich); $50 \mu \mathrm{g} / \mathrm{ml}$ human insulin (Sigma-Aldrich); $0,5 \mathrm{mM}$ isobutyl-

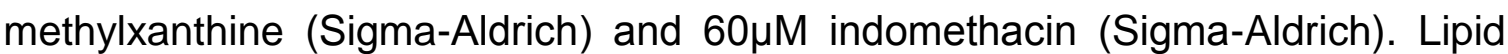
drop formation was evaluated with Oil Red staining (Sigma-Aldrich). 


\subsection{Chondrogenic Differentiation}

Chondrogenic differentiation is achieved by culture medium enrichment with dexamethasone (Sigma), ascorbic acid phosphate (Sigma) with bovine insulin (Sigma), transferrin (Sigma), selenious acid (Sigma), linoleic acid and BSA (Sigma).

Over the period of treatment, there is an up-regulation of chondrogenic markers such as collagen II, collagen XI, aggrecan, perlecan and syndecan. After two weeks of culture, micromass pellets can then fixed and embedded for sectioning and subsequent staining with Safranin-O, toluidine blue or Alcian blue to highlight acid mucopolysaccharides, glycosaminoglycans and proteoglycans, respectively.

To induce chondrogenic differentiation, aliquots of $2.5 \times 10^{5}$ cells were pelleted in polypropylene conical tubes in $0.5 \mathrm{ml}$ of differentiation medium. Pellets were formalin-fixed, embedded in paraffin, examined morphologically and immunostained for Type II collagen (Chemicon Int.; Temecula, CA, USA).

\section{Anti-Tumoral Drugs}

In this work several antitumor agents were used as described in detail in the following Table XIII.

The anti-CD99 0662 monoclonal antibody (MAb) was kindly provided by G. Bernard, INSERM 343, Hospital de l'Archet, Nice, France. Cytotoxic assays with 0662 were performed in collaboration with IOR.

Trabectedin, Zalypsis, Lurbinectidin and PM00113 were kindly provided by PharmaMar, Madrid, Spain, prepared according to manufacturer's instructions and stored at $-20^{\circ} \mathrm{C}$. $1 \mathrm{mg}$ of drug was resuspended in DMSO to the working 
concentration of $100 \mathrm{nM}$ and stored at $-20^{\circ} \mathrm{C}$ for posterior use. Trabectedin and each one of the related compounds chemical structures will be later described and characterized in detail in section results, chapter 2.

Table XIII. Description of the anti-tumoral agents

\begin{tabular}{ccccc}
\hline Compound & MW & Stock & Names & Company \\
\hline ET 743 & 761,8 & $100 \mu \mathrm{M}(\mathrm{DMSO})$ & Trabectedin; Yondelis & Pharmamar \\
\hline PM00104 & 709,71 & $100 \mu \mathrm{M}(\mathrm{DMSO})$ & Zalypsis & Pharmamar \\
\hline PM01183 & 784,28 & $100 \mu \mathrm{M}(\mathrm{DMSO})$ & Lurbinectedin & Pharmamar \\
\hline OSI-906 & 421,5 & $10 \mu \mathrm{M}(\mathrm{DMSO})$ & Linsitinib & Astellas \\
\hline D-4000 & 579,98 & $100 \mu \mathrm{M}(\mathrm{DMSO})$ & Doxorubicin & LC Laboratories \\
\hline O662 & & \multicolumn{1}{c}{ In collaboration with IOR } & \\
\hline O-9201 & 434,46 & $50 \mathrm{nM}(\mathrm{DMSO})$ & Olaparib & LC Laboratories \\
\hline ABT 888 & 244,29 & $50 \mathrm{nM}(\mathrm{DMSO})$ & Veliparib & AxonMEDCHEM \\
\hline I-5432 & 292,03 & $50 \mathrm{nM}(\mathrm{DMSO})$ & Iniparib & LC Laboratories \\
\hline MW stands for Molecular Weight & &
\end{tabular}

Linsitinib or Osi 906 is an IGF1R-IR dual inhibitor, already in clinical trials in ES and also other cancers. This inhibitor blocks both receptors high very affinity. This drug was kindly provided by Astellas under the European project: EUROSARC. Osi 906 was prepared in DMSO upon arrival at stored at $-20^{\circ} \mathrm{C}$.

\section{Ex vivo assays}

Snap frozen samples $(n=9)$ previously diagnosed ES samples from the Tumor Bank of the Hospital Vírgen del Rocío, were used for the Immunohistochemistry $(\mathrm{IHC})$ studies. Samples were diagnosed through the presence of EWSR1 rearrangements and morphological and Histopathological confirmation, according to the WHO classification of 2013. 
IHC was carried out on sections of frozen tissue using the Envision method (Dako, CA, USA) and primary antibodies for CD105, CD90, and CD99 (abcam). IHC staining was evaluated by two pathologists (Table XIV).

Table XIV. Antibodies used for IHQ analysis

\begin{tabular}{ccc}
\hline Primary Antibody & Dilution & Commercial \\
\hline Anti-CD90 & $1: 500$ & Abcam\#ab23894 \\
\hline Anti-CD99 & Ready to use & Dako\#C55233 \\
\hline Anti-CD105 & $1: 500$ & Abcam\# ab44967 \\
\hline Anti-CD45 & Ready to use & Dako\#C55159 \\
Anti-CD34 & $5 \mathrm{mg} / \mathrm{ml}$ & Menarini\#E51635
\end{tabular}

\section{In vivo assays}

\subsection{Xenografts}

Our goal was to study the effectiveness of Trabectedin and Olaparib alone and in combination on NOD/scid mice injected with ES cell lines. The study followed the Spanish and European Union guidelines (RD 1201/05 and $86 / 609 /$ CEE, respectively) and was approved by the ethical committee of our institution

Herein, 4-5 wks-old NOD/scid mice were used. Due to a complex immune deficiency involving B and T lymphocytes, natural killer cells, macrophages and the complement system, NOD/scid mice are almost completely unable to reject human xenotransplants. Induction of tumour xenografts was performed using cell suspensions, containing $5 \times 10^{6}$ alive cells of TC71 in $0.2 \mathrm{ml}$ of $1: 1$ cellular 
medium matrigel matrix (Becton Dickinson; Franklin Lakes, NJ, USA), which were injected subcutaneously into the right flank of the mice.

Mice were randomized into controls and treated groups 1 week after tumour started to be measurable (2-3 weeks after injection; day 0 of treatment), Mice with tumour volumes higher than $1 \mathrm{~cm}^{3}$ were excluded from the analysis. Several treatment groups of 10 mice per group were used: Controls; Trabectedin alone; Olaparib alone; Olaparib plus Trabectedin and finally Trabectedin plus Olaparib.

\subsection{Drug treatment}

Mice received daily injections of study drugs (alone or combined) or physiological saline solution. Tumours were measured every $2-4$ days with a calliper, and diameters were recorded. Tumour volumes were calculated by the formula: $a^{2} b / 2$, where " $a$ " and " $b$ " are the 2 maximum diameters.

Drug Treatments included (Figure 16):

i) Trabectedin: 0,15mg/kg, iv, once a week;

ii) Olaparib: $100 \mathrm{mg} / \mathrm{kg}$, IP twice daily five times per week;

iii) Trabectedin and Olaparib: $100 \mathrm{mg} / \mathrm{kg}$, IP twice daily five times per week + $0,15 \mathrm{mg} / \mathrm{kg}$, iv, once a week;

iv) Olaparib and Trabectedin: Olaparib $100 \mathrm{mg} / \mathrm{kg}$ twice a day, 5 days a week, 3 weeks plus Trabectedin $0,15 \mathrm{mg} / \mathrm{kg}$ once week for two weeks, 7 days after the first dose of Olaparib.

v) Control groups were only treated with vehicle $\mathrm{NaCl} 0,9 \%$ in sterile water milliQ $\mathrm{H}_{2} \mathrm{O}$ IV, vehicle of Trabectedin and 2-hidroxyl-propyl-Bciclodextrin 
$10 \%$ in PBS plus 10\% DMSO (Sigma-Aldrich), intra-peritoneal, vehicle of Olaparib.

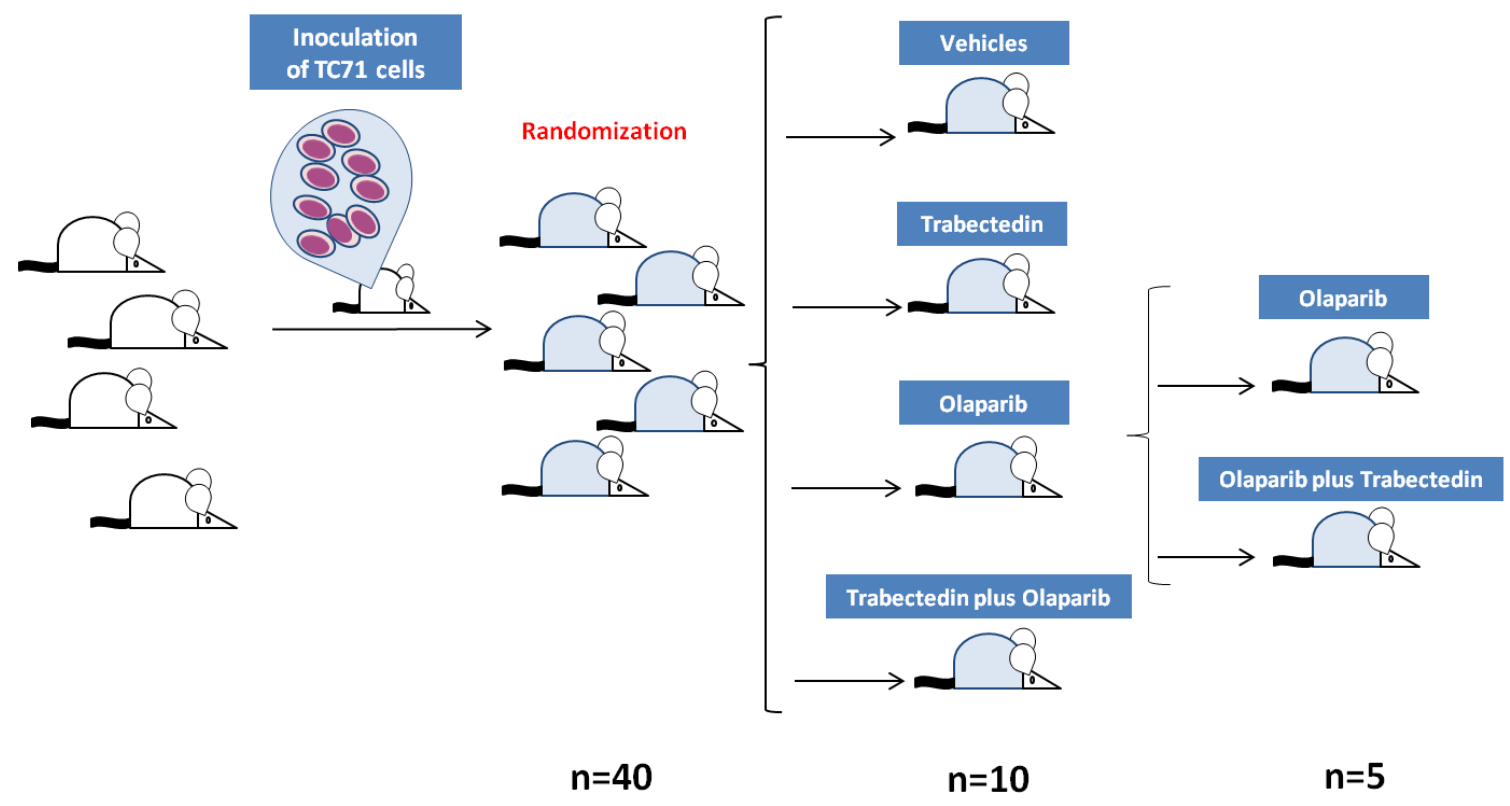

Figure 16. Schematic structure of the in vivo assay. $n=40$ represents the total number of animals at the beginning of the experiment, $n=10$ represents the number of animals per groups and $n=5$ represents the number of animals per group after the division of the initial Olaparib group.

Mice were sacrificed by anaesthesia overdosing 4 weeks after cell injection, and tumours, liver and kidneys were collected for histopathological analysis. All experimental manipulations with mice were performed under sterile conditions in a laminar flow hood.

\subsection{Histopathological analysis}

Extracted tumours, kidney and livers were fixed in formol (Millipore) for 24h and paraffin embedded. Haematoxylin \& eosin staining was performed in all 
tumour samples and the selected kidney and liver samples from each group. An experienced pathologist observed samples in a Leica microscope (Leica).

Haematoxylin \& eosin staining from the in vivo studies were performed with the collaboration of, the Comparative Pathology Laboratory at Centro de Investigación del Cancer, Salamanca.

\subsection{Immunohistochemistry analysis}

Immunohistochemistry was performed using the following antibodies (Table XV). Additionally, TUNEL assay was also performed in the selected tumour samples.

Table XV. Antibodies used for histochemistry analysis in xenografts tumour samples

\begin{tabular}{cccc}
\hline Primary Antibody & Host species & Dilution & Commercial \\
\hline Anti-pH $\mathbf{H}_{\mathbf{2}} \mathrm{Ax}$ & $\mathrm{Ms}$ & $2 \mathrm{ug} / \mathrm{ml}$ & Millipore\#05631 \\
\hline Anti-cPARP & $\mathrm{Rb}$ & $1: 25$ & Cell Signaling\#95421 \\
\hline Anti-BRCA2 & Rbabbit & $1: 200$ & Sigma\#HPA026815 \\
\hline $\begin{array}{c}\text { Anti-c-caspasa 3 } \\
\text { (TUNEL assay) }\end{array}$ & $\mathrm{Rb}$ & $1: 1600$ & Cell Signaling\#9661 \\
\hline
\end{tabular}

\section{Statistical analysis and bio-software}

\subsection{MFC analysis}

MFC analysis was performed using PAWS (SPSS Inc.; Chicago, IL, USA). U-Mann Whitney test was used to look for significant differences between groups ( $p$ values <0.05). 


\subsection{Hierarchical clustering analysis}

Hierarchical clustering analysis was performed using hMSC-ES-P $(n=11)$, hMSC-HD ( $n=6)$, hMSC-AD ( $n=1)$, hMSC-PLD $(n=1)$ and ES cell lines $(n=9)$ in which all markers of the previous panel had been tested. Clustering was run using standard correlation coefficients, for the cluster method, a similarity metric and average linkages were used. The Median Fluorescence Intensity (MFI) value obtained for each tested marker was stored into a database system and a ratio between the MFI obtained for each marker and the mean MFI value obtained for that marker in all samples tested was then calculated. Clustering was performed after median centering and normalizing the fluorescence ratios. A logarithmic (base 2) transformation was applied to the values of this ratio for individual data sets. The resulting normalized log2 ratios were used for further statistical analyses with the J-Express Pro V2.1 software (MolMine AS; Bergen, Norway).

\subsection{Correlation of IC50 and cell lines and determination of Combination Indexes (Cl)}

IC 50 levels of proliferation were determined using the software Origin $\circledast$ (Northampton; MA, USA) whereas Cl was determined using Calcusyn® (Biosoft; Cambridge, UK) software. Calcusyn uses an approximation of the enzyme kinetic models for the evaluation of values of sensitivity to drugs and combination Indexes, the mass-action law. The median-effect principle, derived by Chou, correlates the dose and the effect in the following equation: $f_{a} / f_{b}=\left(D / D_{m}\right)^{m}$ being $f$ the fraction affected by the dose, fb the fraction unaffected $\left(1-f_{a}\right), m$ the slope of the dose effect curve, signifying the sigmoid city of the curve; $D$ the dose of the drug and $D_{m}$ the median effect dose. The linear correlation between the values is 
evaluated by the coefficient $r$, this value should be $>0,90$ for tissue cultures. The software determines the values of $\mathrm{f}_{\mathrm{a}}$, which in this case would be $\mathrm{IC}_{50}$, dose able to determine cell death in half of the population studied.

These values were determined for each drug and each cell line, and after, the combination indexes $(\mathrm{Cl})$ were determined. The $\mathrm{Cl}$ equation is based on the multiple-drug effect equation postulated by Chou-Talalay. In brief this equation determines the levels of synergism, additivity or antagonism between two drugs according to the following equation: $C l=(D)_{1} /\left(D_{x}\right)_{1}+\left(D_{2}\right)_{2} /\left(D_{x}\right)_{2}$, where $C l$ represents Combination Index; D represents the dose; 1 drug 1; 2 drug 2; $x$ the dose used in the combination. When $\mathrm{Cl}$ is below 1 , represents synergism; $\mathrm{Cl}=1$ represent additivity and $\mathrm{Cl}>1$ represent antagonism. From these models we obtained the combination indexes, the normalized isobolograms, the dose reduction effect and the isobolograms. 


\section{Results}


The Pathogenesis of Ewing Sarcoma/ Results 
1. Immunophenotype and its implication in ES biology and treatment Targeting the CD99 membrane receptor (Amaral AT et al, PloS One, 2014)

2. Comparative studies on Marine compounds Trabectedin, Zalypsis and Lurbinectedin activity in Ewing Sarcoma Pharmacodynamic studies in ES.

(Amaral AT \& Ordoñez JL et al, Submitted, 2014)

3. Trabectedin efficacy in ES is greatly increased by combination with anti-IGF signaling agents

Combination of Linsitinib with Trabectedin.

(Amaral AT \& Garofalo C et al, Submitted, 2014)

4. PARPi Olaparib enhances sensitivity of Ewing Sarcoma cells to Trabectedin: An in vitro and in vivo assay A new promising therapy in ES.

(Ordoñez JL \& Amaral AT et al, Submitted, 2014) 
The Pathogenesis of Ewing Sarcoma/ Results 


\section{1. Immunophenotype and its implication in ES biology and treatment}

Targeting the CD99 membrane receptor Adapted from Amaral AT et al, PloS One, 2014 


\section{Abstract}

ES is a mesenchymal-derived tumor that generally arises in bone and soft tissue. Intensive research regarding the pathogenesis of ES has been insufficient to pinpoint the early events of Ewing sarcomagenesis. However, the MSC is currently accepted as the most probable cell of origin. In an initial study regarding an in depth characterization of MSC-P, we compared them with MSC-HD and ES cell lines.

We evaluated the presence of the EWSR1-FLI1 gene fusion and EWSR1 gene rearrangements in MSC-EP. The presence of the ES transcript was confirmed by q-RT-PCR. In order to determine early events possibly involved in malignant transformation, we used a multiparameter quantitative strategy that included both MSC immunophenotypic negative/positive markers, and ES intrinsic phenotypical features. Markers CD105, CD90, CD34 and CD45 were confirmed in ES samples. We determined that MSC-P lack the most prevalent gene fusion, EWSR1-FLI1 as well as EWSR1 gene rearrangements.

Our study also revealed that MSC-P are more alike to MSC-HD than to EWS cells. Nonetheless, we also observed that EWS cells had a few overlapping features with MSC. As a relevant example, MSC also showed CD99 expression, a hallmark of ES diagnosis. However, we observed that, in contrast to ES cells, MSC were not sensitive to the inhibition of CD99. In conclusion, our results suggest that MSC from ES patients are phenotypically similar to MSC-HD and different from ES cells. This raises important questions regarding MSC role in sarcomagenesis. 


\subsection{Characterization of MSC}

All samples were fully characterized according to the ISCT regarding i) differentiation potential, ii) plastic adherence and iii) determination of positive/negative surface antigens.[21] After 21 days of treatment with adipogenic differentiation medium, lipid droplets were observed, confirming their potential to differentiate into adipoblasts (Figure $17 \mathrm{~A}$, upper left). MSC were also able to differentiate into osteoblasts which display alkaline phosphatase activity together with calcium deposits (Figure 17A, bottom). In addition, MSC showed chondrogenic ability, as confirmed by strong collagen type II positivity (Figure 17A, upper right). All MSC presented plastic adherence. MSC-HD and MSC-P had a similar growth rate as observed in Figure 17B.

A.

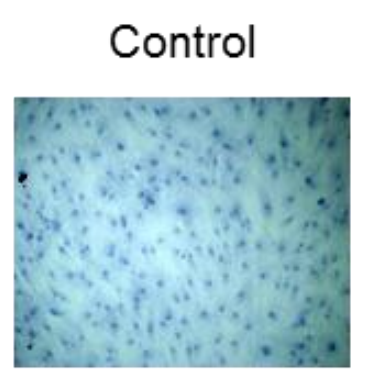

Adipogenic

Lineage

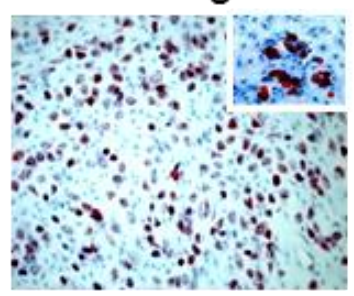

Chondrogenic

Control

Osteogenic Lineage
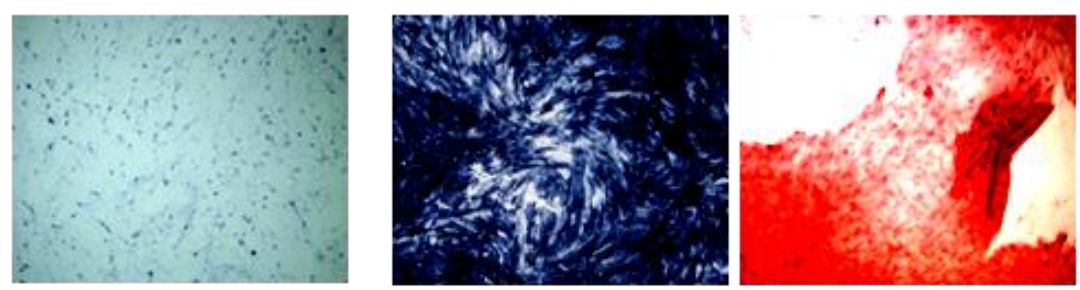
B

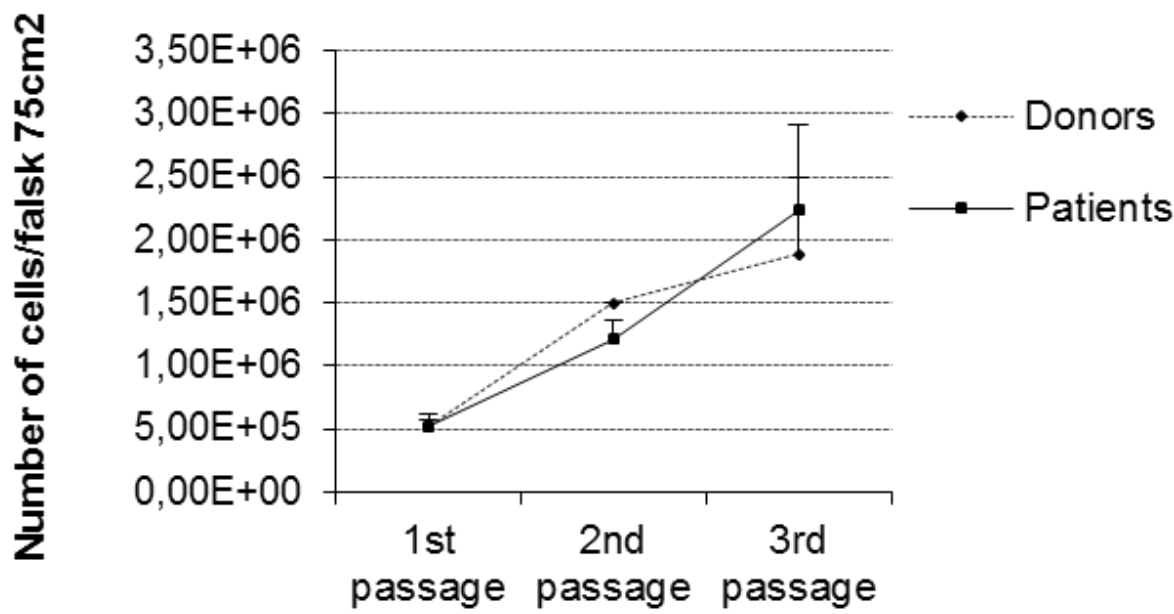

Figure 17. Characterization of MSC. (A) In vitro differentiation potential of MSC. In vitro adipogenic differentiation. Representative images show the light microscopic view of lipid drop accumulation after 3 weeks of culture in control medium and in differentiation induction medium. In vitro chondrogenic potential of MSC. Representative images show the light microscopic view of alkaline phosphatase activity (detected by a blue-dark staining) and mineralization after culture in control medium and in differentiation induction medium (detected by red staining). (B) Growth rate evaluation in MSC-HD and MSC-P. Cells were counted with trypan blue at each passage. Trypan blue selectively colors dead cell in blue whereas live cells are observed shinning since this marker is unable to enter the cytoplasm when the cell membrane in intact. Both sets of samples presented similar growth rates and were in continuous growth during these experiments.

Moreover, the samples exhibited a positive expression for the markers: CD105, CD90, CD73 as well as the adhesion molecule CD166. Conversely, the hematopoietic lineage markers CD34, CD45, CD19 and HLA-DR were not found (Figure 18). 

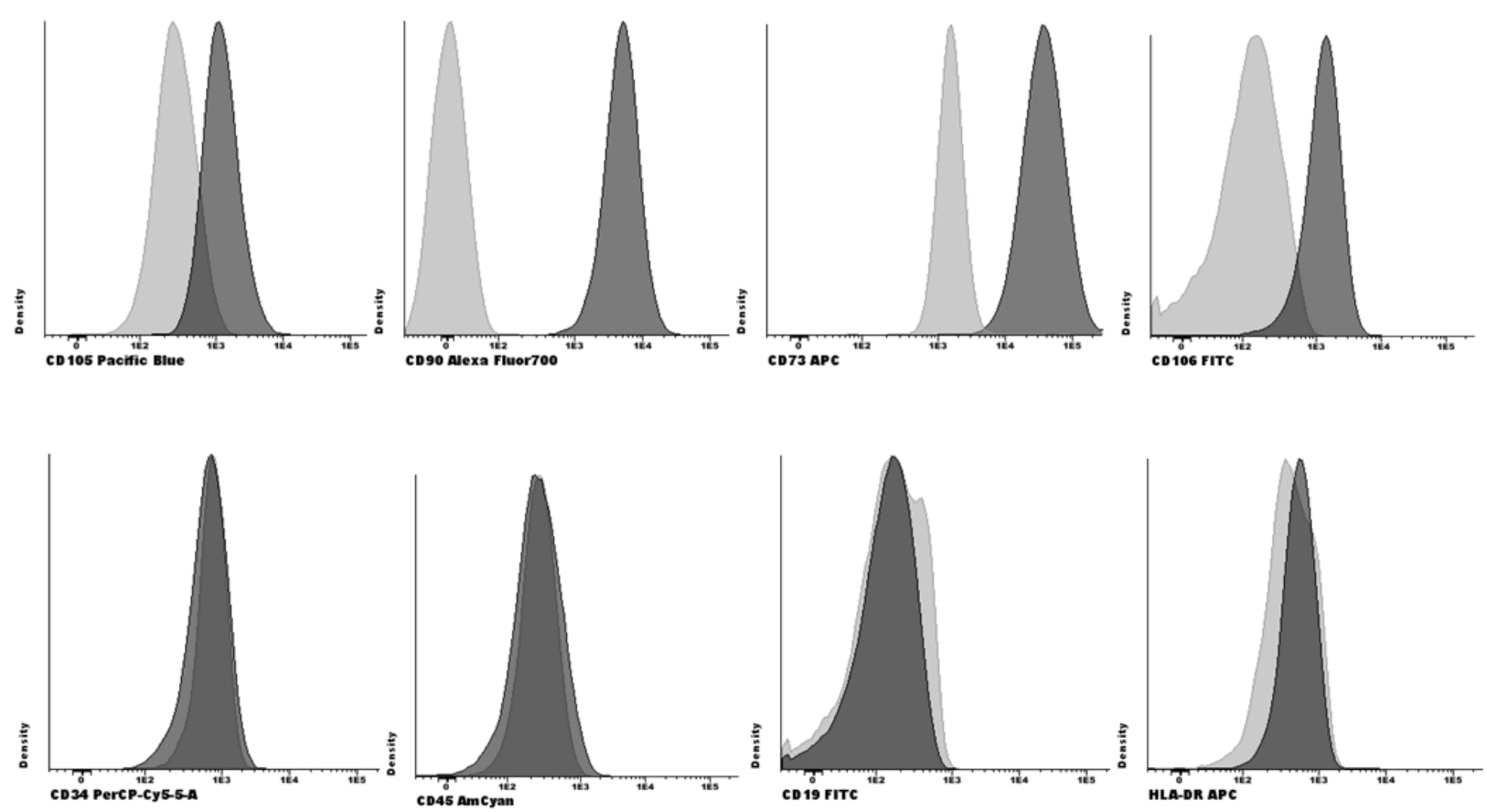

Figure 18. Immunophenotypic characterization of MSC: positive expression of CD105, CD90, CD73 and CD106 and negative expression of hematopoietic markers CD34, CD45, CD19 and HLA-DR (light gray represents control and darker gray represents sample with antibody).

\subsection{MSC derived from EWS Patients lack EWSR1 gene rearrangements}

We next explored the presence of the EWSR1-FLI1 gene fusion in MSC-P in all cases. We observed that they lacked this chimeric gene (Figure 19A, pictures 1 and 2). Subsequently, we assessed the presence of possible rearrangements of the EWSR1 gene. We observed that MSC-P had no rearrangements of this gene (Figure 19A, pictures 4 and 5). As expected, the ES cell lines showed a break on the EWSR1 gene and the fusion gene EWSR1-FLI1 (Figure 19A, pictures 3 and 6, respectively), therefore these were used as a control. In order to validate this result we also performed qRT-PCR for the various ES transcripts in BM-hMSC samples. In Figure 19B, it may be observed that in comparison to ES cell lines bearing the different transcripts: TC71 
(EWSR1-FLI-1-type 1; Cado-ES (EWSR1-ERG); RDES (EWSR1-FLI1-type 2) and A4573 (EWSR1-Fll1-type 3), MSC-P did not show these transcripts.

A

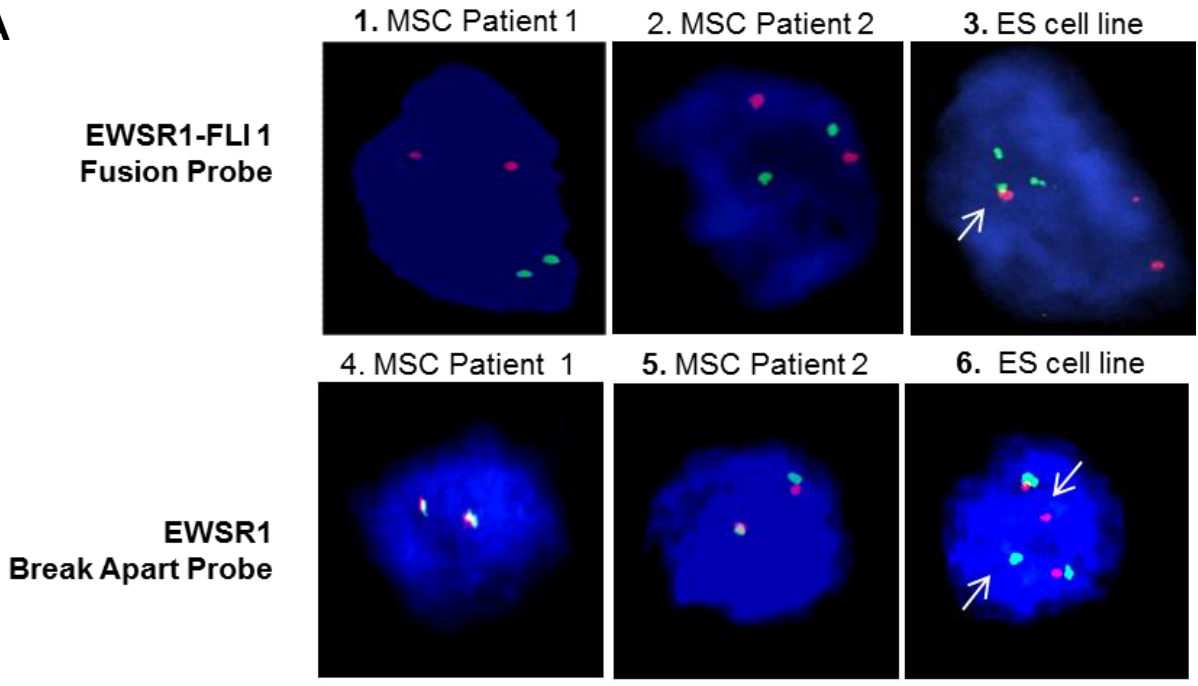

2. MSC Patient 2

3. ES cell line

B

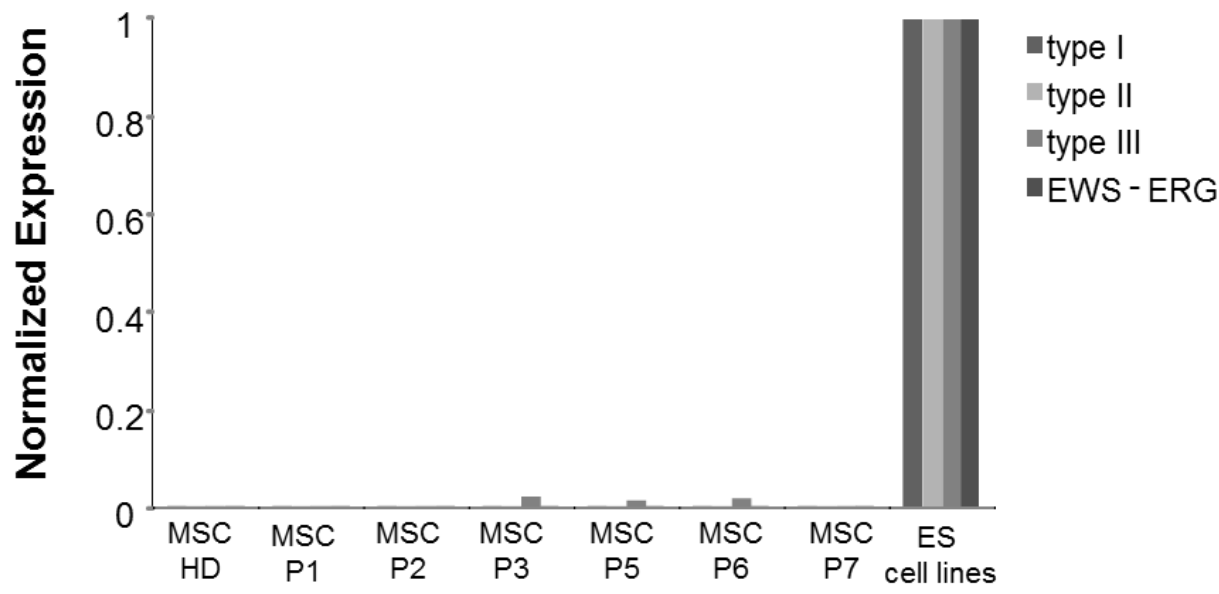

Figure 19. MSC-P lack the presence of the EWSR1-FLI1 chromosomal translocation. (A) Study of the chromosomal translocation between genes EWSR1 and FLI1 using a EWSR1-FLI 1 homemade-fusion probe (1-3). MSC-P showed two normal copies of both EWSR1 and FLI1 (1 and 2) whereas in the TC71 cell line we observed not only two normal copies but also one EWSR1-FLI1 fusion, marked by a white arrow (3) Rearrangements of the EWSR1 gene were studied using a EWSR1 break-apart probe.(4-6) Representative images of MSCP, ES-P-01 and ES-P-02, ( 4 and 5) failed to present a break in the EWSR1 gene, whereas the positive control here represented by the TC71 cell line (6), showed a distinctive rearrangement of this gene, marked by white arrows. (B) Results were validated by q-RT-PCR using ES cell lines as a positive control. MSC-P, here represented by Patients $1 ; 2 ; 3 ; 5 ; 6$ and 7 as well a healthy donor display a clear negative expression of all the transcripts. 


\subsection{MSC derived from ES Patients have a low expression of ES markers}

The precise immunophenotypic profile of ES cells had never been fully established. Here, we analyzed the expression of CD271, CD54 and CD117 in a panel of ES cell lines (Figure 20). CD271, the low affinity nerve growth factor receptor, displayed a heterogeneous expression pattern among the different ES cell lines (Figure 20).

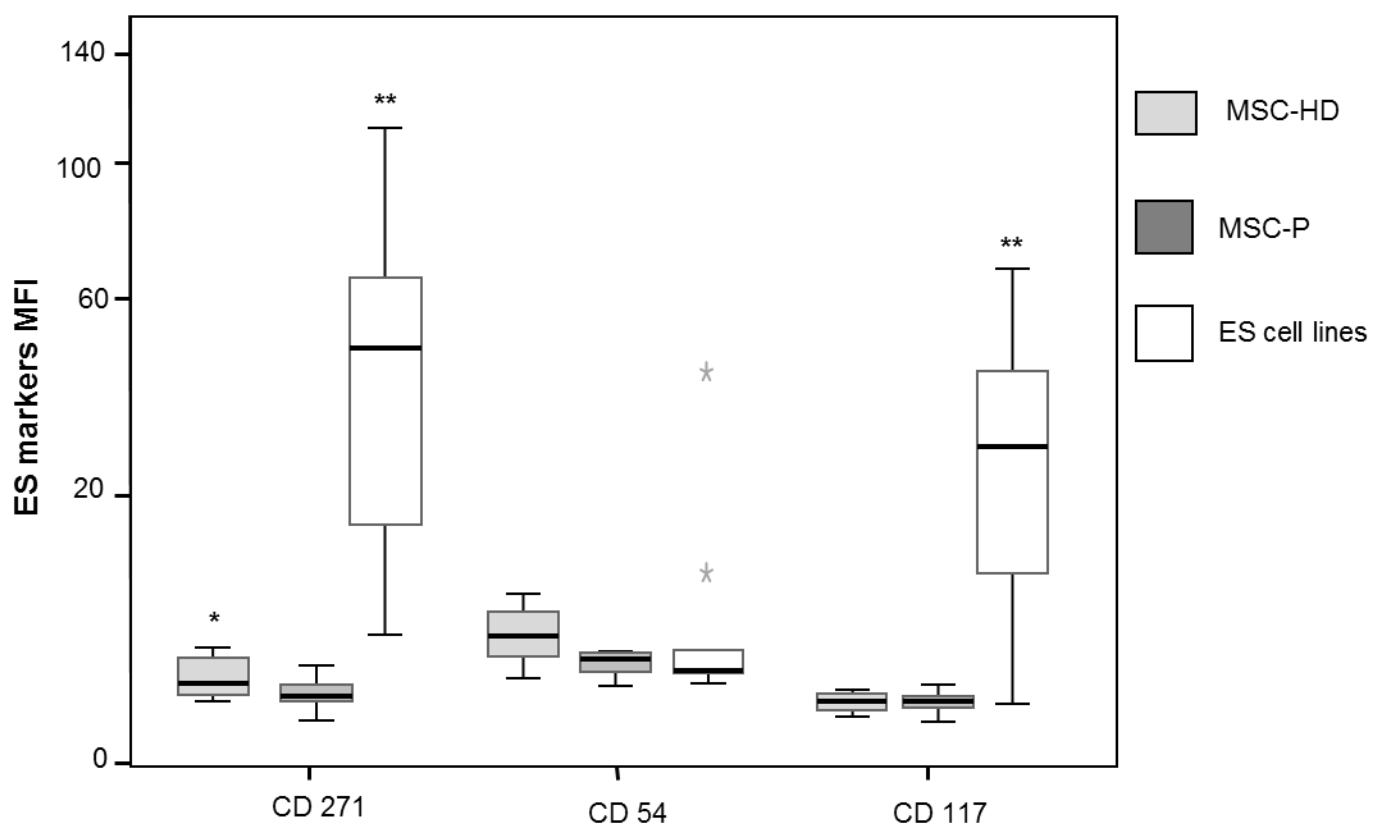

Figure 20. Immunophenotype of MSC-P versus ES cells. Comparative study between MSC-HD and MSC-P regarding ES markers: CD271, CD54 and CD117. The expression levels of the EWS markers CD271, CD54 and CD117 were evaluated by MFC and medium values of MFI are represented by groups. (* represent $\mathrm{p} U-\mathrm{Mann}$ Whitney $<0.05$ for MSC-P versus MSC-HD and ${ }^{* *} p \mathrm{U}-\mathrm{Mann}$ Whitney $<0.001$ for MSC-P versus ES cell lines). All ES cell lines present positive expression of CD271, when compared to the control. ES cell lines show weak expression of CD54 when compared to the control, with the exception of RDES and STAET1 cell lines with strong positive expression values. ES cell lines present a heterogeneous expression of CD117.

CD271 has also been reported to be expressed in MSC. In this sense, here we observed a significantly higher expression of CD271 in MSC-HD as 
compared to MSC-P $(p=0.01)$ (Figure 20). However, it is worth noting that both MSC-HD and MSC-P showed significantly lower levels of this marker than ES cell lines $(p<0.05)$ (Figure 20). CD54 (ICAM-1), an adhesion molecule involved in cell-matrix-cell interactions, was weakly expressed by all ES cell lines, with the exception of RDES and STAET 1 (Figure 20). Similarly, CD54 was weakly expressed in MSC, however it was slightly more expressed in MSC-HD compared to MSC-P (Figure 20). CD117 (C-KIT) has been extensively described as a tyrosine-kinase receptor overexpressed in most ES cell lines and ES tumor samples. $[96,120,121]$

Consistently, most of the ES cell lines studied here displayed high CD117 expression levels (Figure 20). C-KIT, showed a similar expression in MSC-HD and MSC-P, but its expression was significantly lower than that in the ES cell lines $(p=0.001)$ (Figure 20).

\subsection{The plasma membrane expression pattern of ES cell lines is globally different from that of MSC}

After studying the presence of ES markers in MSC, we performed the opposite approach, in which we evaluated the presence of an exhaustive panel of MSC markers in ES cell lines $(n=9)$. We performed a hierarchical unsupervised cluster analysis to compare the expression of MSC-positive markers, MSCnegative markers and ES markers in all samples. We observed a clear division into two clusters, one consisting of all the ES cell lines and another consisting of all MSC samples $(n=19)$, which included HD, ES-P, and a set of controls, Adipose and Placenta derived MSC (Figure 21). 


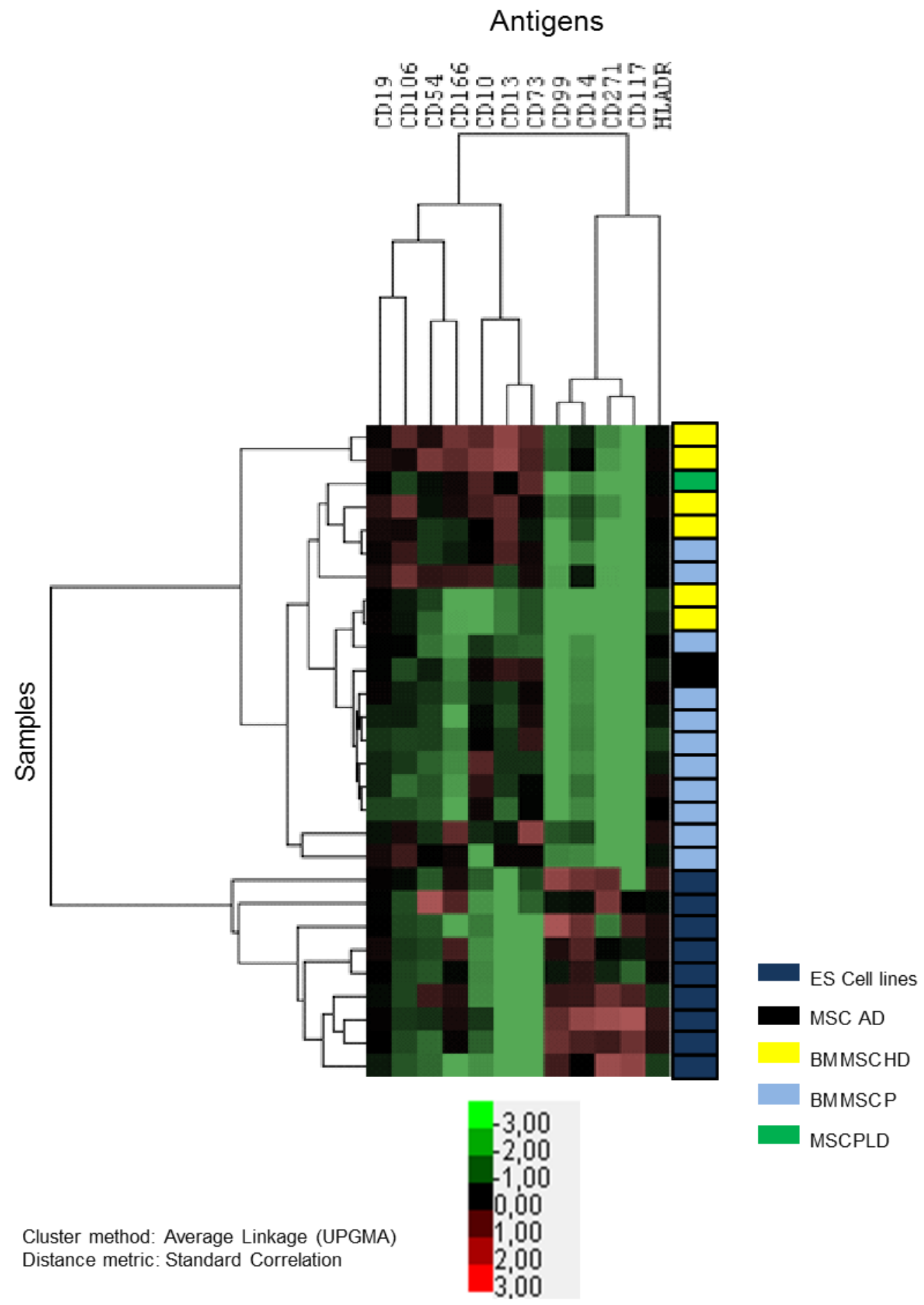

Figure 21. Comparative study between MSC, ES cell lines and ES samples. Hierarchical unsupervised cluster analysis showed two different clusters, one consisting of all ES cell lines and the other of all MSC samples. Control was performed using Fluorescence minus one. The following markers: CD105, CD34, CD45 and CD90 were used in every tube in order to diminish the autofluorescence presented by MSC, therefore these were not considered in this analysis, and were validated in frozen tissue from ES samples. 
Additionally, we evaluated the expression of CD90, CD105, CD45 and CD34 one by one in a set of ES samples $(n=9)$ by IHC. The ES samples presented the typical small round cell morphology and strong CD99 expression as observed on Figure 22 top panel. CD90 was strongly expressed in all ES samples. CD105 was positively expressed in tumor cells and strongly expressed in the cells surrounding the tumor vessels (Figure 22 middle panel).

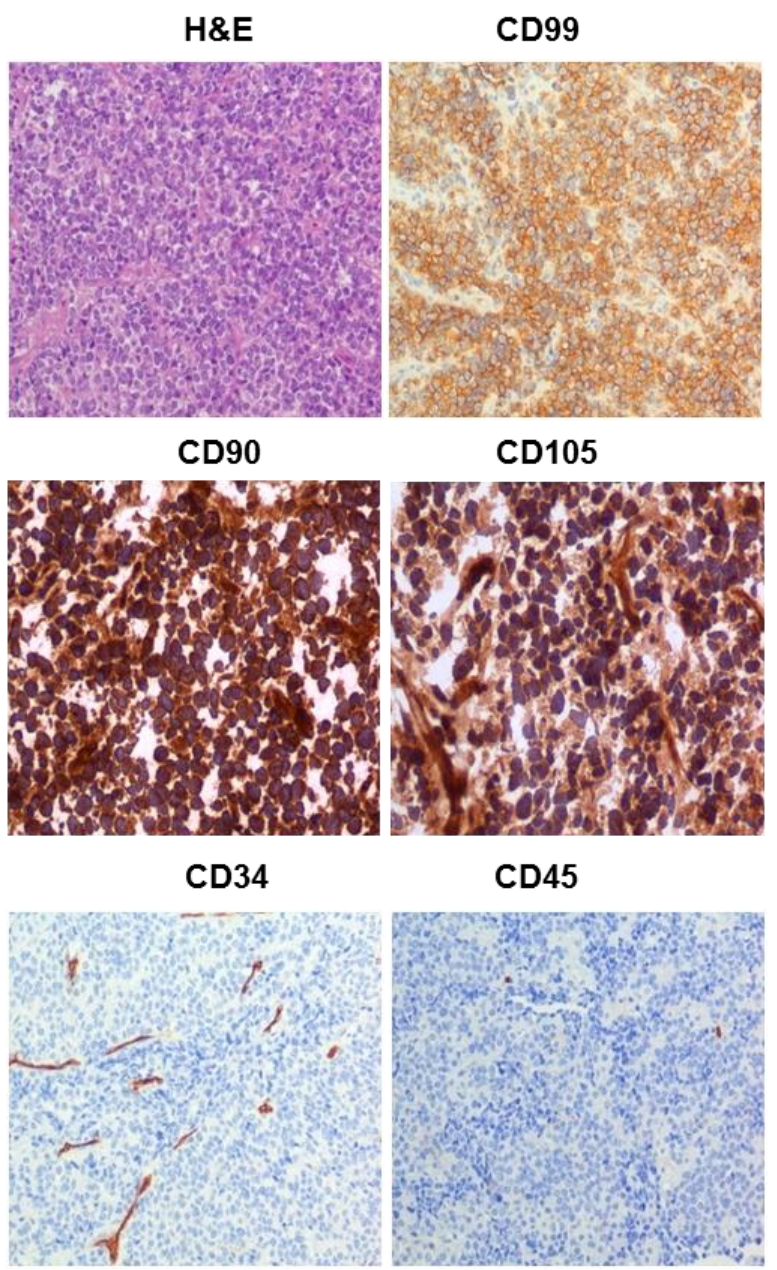

Figure 22. IHC studies in frozen tissue from ES samples. Upper panel represents ES typical morphology as well as CD99 strong expression in the plasma membrane of tumor cells. Middle panel shows strong expression of CD90 and positivity for CD105. Finally, lower panel shows ES negative expression of both CD45 and CD34 
Regarding hematopoietic markers CD34 and CD45, all EWS samples showed negative expression in the tumor cells (Figure 22 lower panel). Results were homogeneously observed in all ES samples analyzed. Expression of CD90, CD105, CD34 and CD45 in EWS cell lines, in comparison with MSC-HD and MSC-P is detailed on Figure 23.
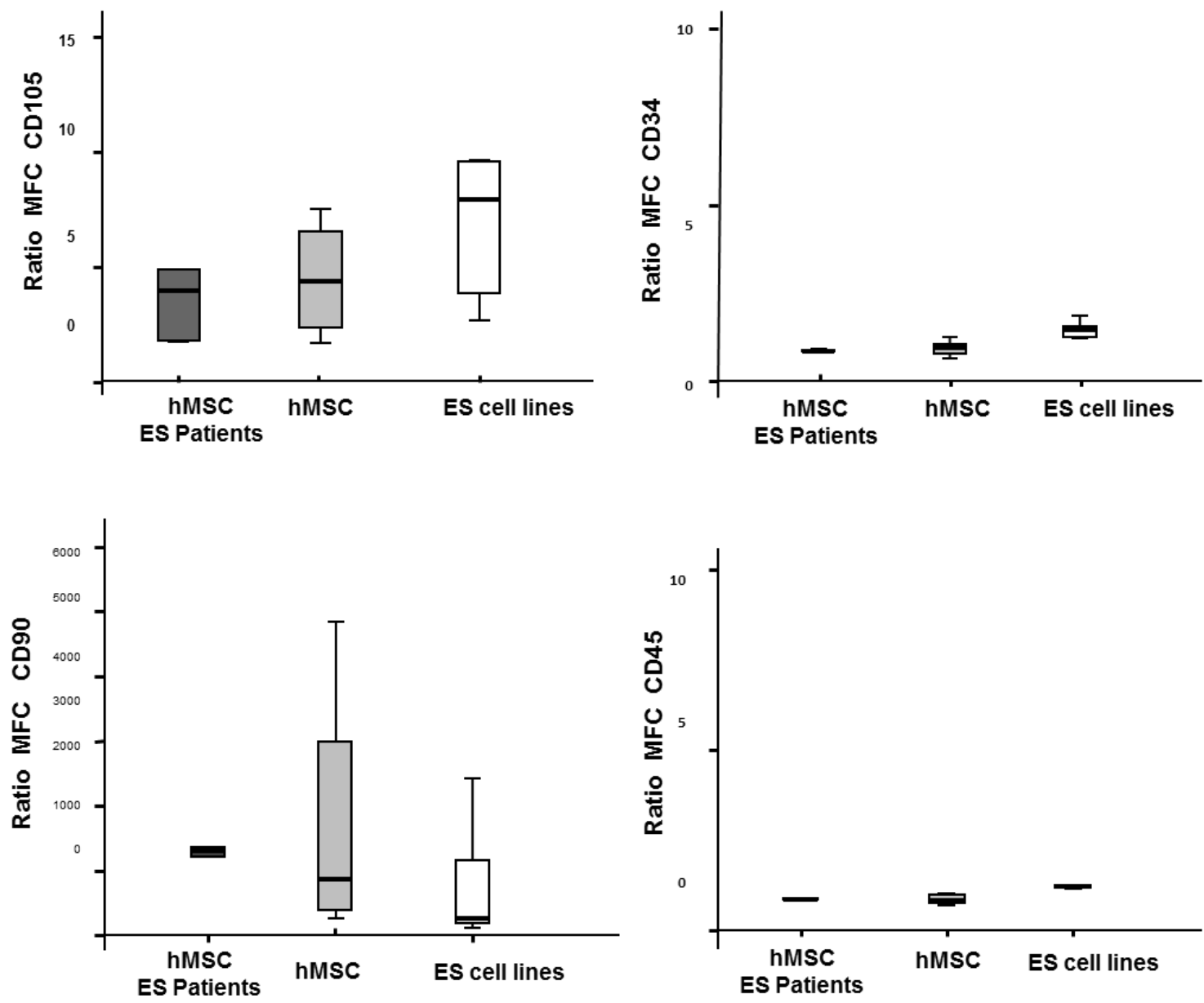

Figure 23. Expression of CD105, CD34, CD90 and CD45. CD105 is differentially expressed between cell lines and MSC. CD34, hematopoietic marker is under expressed in MSC and in ES cell lines. CD90 is highly expressed in all samples. CD45 is under expressed in MSC and ES cell lines. 


\subsection{MSC derived from ES patients showed lower levels of CD99 expression}

\section{than that of ES cells}

CD99 transmembrane glycoprotein overexpression is routinely used as a diagnostic marker of ES. Herein, we observed that MSC-P presented low to moderate levels of CD99 expression, similarly to MSC-HD and significantly lower than ES cell lines ( $p=0,001)$ (Figure 24).

A

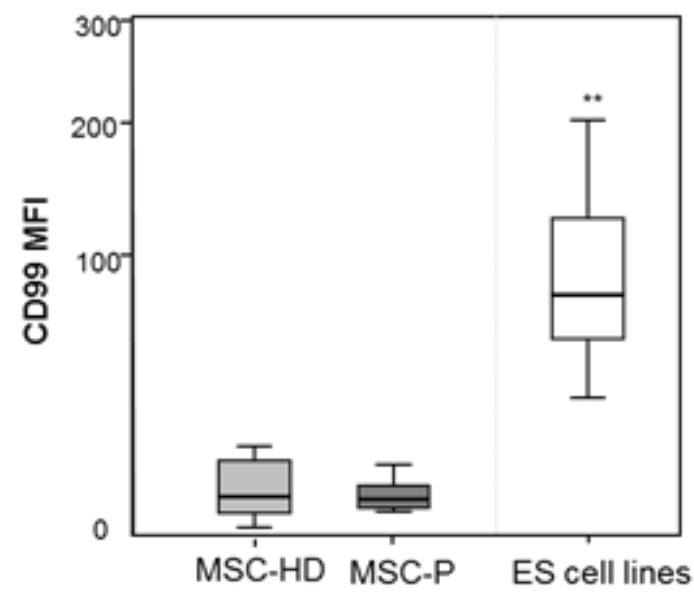

B

\begin{tabular}{ccc}
\hline $\begin{array}{c}\text { ES cell } \\
\text { line }\end{array}$ & $\begin{array}{c}\text { CD99 } \\
\text { MFI }\end{array}$ & $\begin{array}{c}\text { ES fusion } \\
\text { type }\end{array}$ \\
\hline A4573 & ++ & EWSR1-FLI 1 \\
A673 & +++ & EWSR1-FLI 1 \\
Cado-ES & +++ & EWSR1-ERG \\
RD-ES & + & EWSR1-FLI 1 \\
RM82 & ++ & EWSR1-ERG \\
Staet 1 & ++ & EWSR1-FLI 1 \\
Staet 2.1 & ++ & EWSR!-FLI 1 \\
Staet 10 & +++ & EWSR1-FLI 1 \\
TC 71 & ++ & EWSR1-FLI 1 \\
\hline
\end{tabular}

Figure 24. CD99 expression in MSC and ES cell lines. (A) CD99 differential expression between MSC-HD, MSc-P and ES cell lines. (B)Table 1 CD99 expression in ES cell lines. ES cells presented a rather heterogeneous pattern; nonetheless all cell lines studied here were positive for CD99 expression. Cell lines such as A673 and Cado-ES presented higher levels (score +++) of CD99 expression, whereas RDES cell line presented the lowest levels (score + ) of expression. This situation was analyzed with comparison to a control which is the median of all tubes without antibody and intensity levels were calculated with ration between sample and control. (Ratio $<1$ - -; ratio around 1, - ; ratio=1, +/-, ratio $>2+$, ratio $>10++$, ratio $>100+++$ ). (C) CD99 expression in MSC-P and ES cell lines. CD99 expression is variable within both groups and similar between the two groups of MSC samples, ranging from weak to moderate, and always lower that the ES cell lines tested here. ( ${ }^{*} p$ Mann-Whitney $<0.05$ ES cell lines versus MSC and MSC-P versus ES cell lines). 
As depicted in Table XVI, MSC-P presented comparable intensity levels of CD99 expression between them. Distance to the primary tumor did not seem to impact CD99 expression by hMSC. Interestingly, patients MSC-P-05 and MSC-P11 presented the primary tumor in the same bone from which MSC were collected (iliac bone). Despite being closer to the primary tumor, these MSC presented comparable levels of CD99 intensity to the other patients. MSC-P presented CD99 levels of expression lower than ES cell lines, with the exception of the RDES cell line, which presented the lowest CD99 intensity (Figure 24 B).

\subsection{CD99 was heterogeneously expressed in MSC from different tissue} sources

We extended our study on CD99 expression to MSC derived from different sources of healthy tissues (BM, iliac crest, amnios, chorion, dental pulp, adipose tissue, and placenta). In this new set of BM-MSC samples, we observed that CD99 expression was also quite variable, ranging from negative to overtly positive (Table XVII). Regarding MSC from other healthy tissues, all samples with the exception of 2 amnios and 2 adipose derived samples, presented positive CD99 expression (Table XVII). Overall, weak to highly positive expression of CD99 was found in 17 out of $23(71 \%)$ MSC samples. Of these, at least $11 \mathrm{MSC}$ (scored as ++ and +++ ) showing expression levels similar to those found in ES cell lines. 
Table XVI. CD99 intensity levels on MSC-P as assessed by Multiparameter Flow Cytometry

\begin{tabular}{lclllll}
\hline Sample & CD99 $^{\text {MFI }}{ }^{1}$ & PN $^{2}$ & Age & Fusion type & Primary tumor & Remarks \\
\hline MSC-P-01 & + & P1 & 12 & EWSR1-FLI1 & Humerus & - \\
MSC-P-02 & + & P2 & 15 & EWSR1 BA ${ }^{3}$ positive & Intramuscular & - \\
MSC-P-03 & + & P3 & 18 & EWSR1-FLI1 & Humerus & - \\
MSC-P-05 & + & P1 & 17 & EWSR1-FLI1 & lliac crest & - \\
MSC-P-07 & + & P2 & 20 & EWSR1 BA ${ }^{3}$ positive & Fibula & - \\
MSC-P-08 & $+/-$ & P2 & 17 & EWSR1 BA ${ }^{3}$ positive & Fibula & - \\
MSC-P-09 & + & P3 & 24 & EWSR1 BA ${ }^{3}$ positive & Rib & - \\
MSC-P-10 & + & P2 & 21 & EWSR1 BA ${ }^{3}$ positive & Th7 & - \\
MSC-P-11 & $+/-$ & P2 & 29 & EWSR1-FLI1 & lliac crest & MD $^{4}$ \\
MSC-P-12 & $+/-$ & P3 & 19 & EWSR1 BA ${ }^{3}$ positive & L1 vertebra & MD $^{4}$ \\
MSC-P-13 & + & P2 & 31 & EWSR1 BA ${ }^{3}$ positive & Humerus & -
\end{tabular}

${ }^{1} \mathrm{MFI}$ stands for Median Fluorescence Intensity, ${ }^{2} \mathrm{PN}$ stands for Passage Number; ${ }^{3} \mathrm{BA}$ stands for Break Apart; ${ }^{4} \mathrm{MD}$ stands for Metastasis at diagnosis. All samples were positive for CD99 with low to moderate levels of intensity. Samples were analyzed at low passages (1-3). Primary tumors were located in distinct locations and all presented EWSR1 gene rearrangements and EWSR1-FLI 1 fusion in some cases. This situation was analyzed with comparison to a control which is the median of all tubes without antibody and intensity levels were calculated with ratio between sample / control. (Ratio <1- -; ratio around 1 -; ratio=1, $+/-$, ratio $>2+$, ratio $>10++$, ratio $>100+++$ ). 
Table XVII. CD99 expression in MSC derived from healthy tissues

\begin{tabular}{|c|c|c|c|}
\hline Sample & CD99 MFI ${ }^{1}$ & $\mathbf{P N}^{2}$ & Localization \\
\hline BM-DN-TD & ++ & $\mathrm{P} 4$ & Iliac crest \\
\hline BM-DN-HB & ++ & P5 & Iliac crest \\
\hline BM-DN-001 & +++ & P6 & Iliac crest \\
\hline BM-DN-005 & +++ & P6 & Iliac crest \\
\hline BM-DN-HU-1 & + & P4 & Iliac crest \\
\hline BM-DN-HU-2 & + & P4 & Iliac crest \\
\hline BM-DN-TD2 & ++ & $\mathrm{P} 4$ & Iliac crest \\
\hline DP-CM & ++ & P5 & Dental Pulp \\
\hline DP-15 & ++ & P6 & Dental Pulp \\
\hline DP-49 & ++ & P6 & Dental Pulp \\
\hline COR-3442 & + & P5 & Chorion \\
\hline COR-3386 & + & P5 & Chorion \\
\hline COR-3412 & + & P6 & Chorion \\
\hline AM-3481 & - & P6 & Amnios \\
\hline AM-3386 & $+/-$ & P6 & Amnios \\
\hline AM-3396 & - & P5 & Amnios \\
\hline PLD-2 & ++ & P4 & Placenta \\
\hline PLD-3 & ++ & P4 & Placenta \\
\hline PLD & + & P5 & Placenta \\
\hline AD-HADAS & $+/-$ & $\mathrm{P} 4$ & Adipose Tissue \\
\hline AD-HADAS-1 & - & P3 & Adipose Tissue \\
\hline AD-ADIPO3412 & - & P5 & Adipose Tissue \\
\hline AD-002 & ++ & $\mathrm{P} 4$ & Adipose Tissue \\
\hline
\end{tabular}

${ }^{1} \mathrm{MFI}$ stands for Median Fluorescence Intensity, ${ }^{2} \mathrm{PN}$ stands for Passage Number. CD99 expression levels in MSC derived from normal tissue, including BM; dental pulp; adipose tissue; chorion; amnios and placenta. Again, intensity levels for CD99 expression were variable with 11/19 samples presenting moderate/high expression (scores ++ and +++); 8/19 presenting low levels of expression and 4/19 with negative CD99 expression. Samples were studied between passages 4 and 6 . 


\subsection{CD99 engagement does not elicit impairment of MSC survival}

We evaluated whether engagement of CD99 with $0662 \mathrm{MAb}$ was able to induce apoptosis as previously shown in ES cells and BM-MSC (Figure 25) Cell cultures from different BM-MSC $(n=5)$ positive for CD99 at similar levels, were exposed to anti-CD99 MAb and RDES was used as a control.
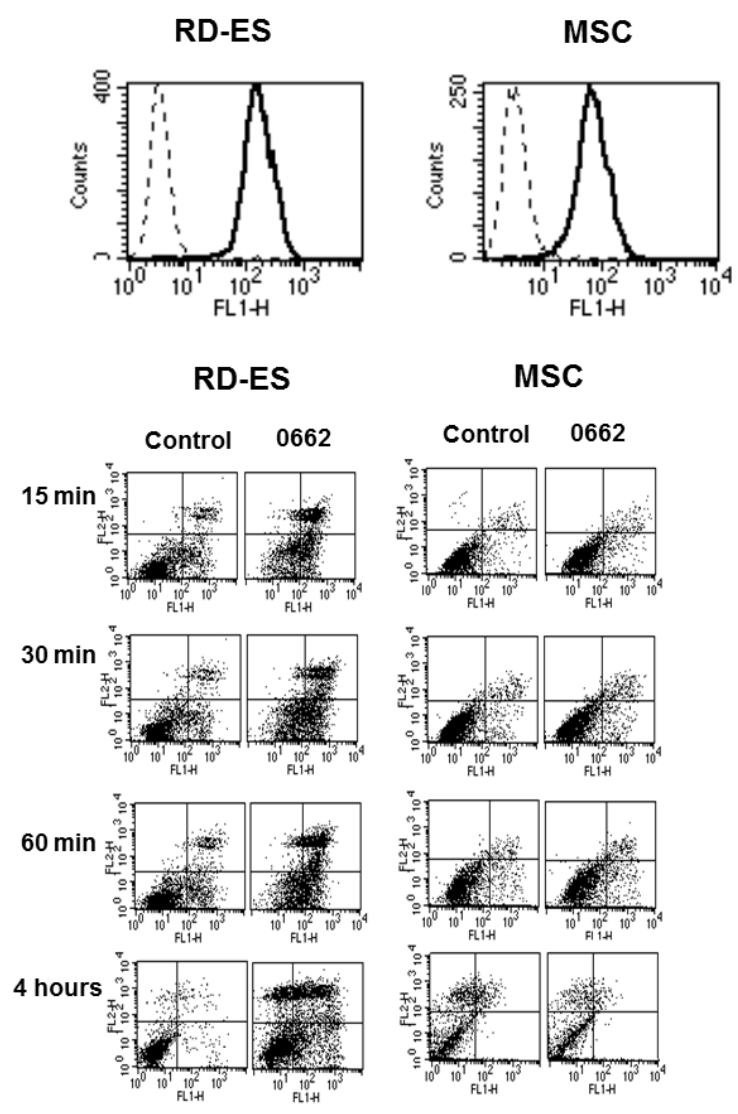

Figure 25. Cytofluorimetric analysis of CD99 expression on RD-ES cell line and BM-MSC. A) Dotted line represents cells stained with secondary antibody alone; solid profile represents cells stained with anti-CD99 antibody. In each panel, the ordinate represents the number of cells. All the $5 \mathrm{MSC}$ used for functional assays presented similar levels of CD99 expression. (B) Time-course analysis of apoptosis after exposure to anti-CD99 0662 MAb. RD-ES is used as positive control. Cell death was determined by examining annexin V-PI binding with flow cytometry. Early-apoptotic cells were annexin V-positive and PI -negative (LowerRight region). Late apoptotic/necrotic cells were annexin V- and PI-positive (Upper-Right region). Results shown here are representative of all the 5 CD99highly positive MSC. 
We observed that a mere 15 minute-treatment was enough to induce a 27 $\%$ of apoptotic cells in RDES $(p<0.05)$, whereas the same treatment regimen only triggered a $7 \%$ of apoptotic population in MSC (Figure 26). Furthermore, 4 hours of treatment lead to an almost complete apoptotic RDES culture (73\% annexin-V positive cells) $(p<0.05)$ but failed to increase significantly the initial percentage of apoptosis in MSC (Figure 26).

A

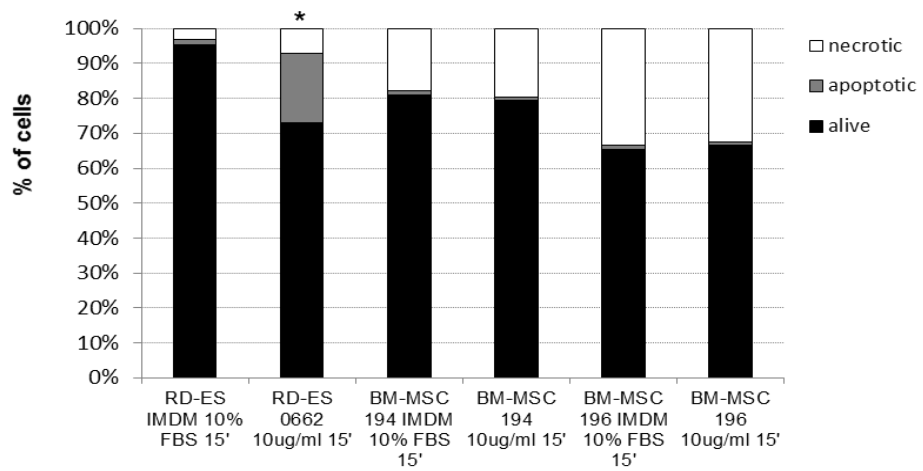

B

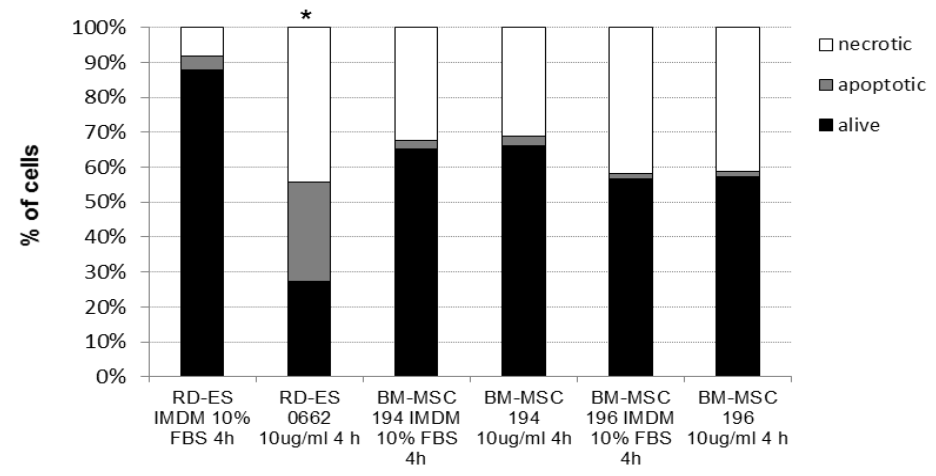

Figure 26. Percentage of living, apoptotic and necrotic cells observed in cells (two representative cultures of BM-MSC and RD-ES ES cell line) treated with anti-CD99 MAb for $15 \mathrm{~min}$ or 4hrs. (* represents statistical significance in comparison to control $p<0.05)$ The assay was performed on detached cells. The ES cell line shows an increment in the percentage of apoptotic and necrotic cells after CD99 engagement, whereas the BM-MSC show similar percentages of alive and dying cells in treated or untreated conditions. * represents statistical significance as compared to control $p<0.05$ 
The Pathogenesis of Ewing Sarcoma/ Results 
2.

Comparative studies on Marine compounds Trabectedin, Zalypsis and Lurbinectedin activity in Ewing Sarcoma

\section{Pharmacodynamic studies in ES}

Adapted from Amaral AT and Ordoñez J et al, Submitted, 2014 


\section{Abstract}

Ewing Sarcoma is a developmental sarcoma of the bone and soft tissues. Trabectedin, a natural compound isolated from the sea, has been reported as one of the most potent in vitro cytotoxic agents towards ES cell lines.

Herein, we studied the sensitivity of a large panel of ES cell lines to Trabectedin and compared it to the related compounds: Zalypsis and Lurbinectedin. We observed that all agents are highly active in terms of proliferation inhibition, apoptosis induction and cell cycle arrest. Also, we explored the capacity to form Double strand breaks in the DNA and the consequent DNA damage induction and DDR pathway activation. We also observed that one cell line resistant to Trabectedin also showed resistance to the other two compounds.

As a conclusion, Zalypsis and Lurbinectedin proved to be highly active in vitro, which can be very appealing in the clinic given that these compounds present different pharmacokinetic parameters as well as distinct secondary events when compared to Trabectedin. 
2.1 Trabectedin inhibits proliferation in all ES cells at the sub-nanomolar range

Analysis of cell proliferation after 72 hours of treatment revealed that all ES cell lines are extremely sensitive to Trabectedin. We observed a median of IC 50 of around $90.05 \pm 43.3 \mathrm{pM}$. We studied the possible correlation between the fusion type harbored by each cell line/status of p53 and sensitivity to Trabectedin. We found that there is no correlation between these factors and the $\mathrm{IC}_{50}$ levels to Trabectedin (Mann-Whitney U test) (Table XVIII).

Table XVIII. Proliferation IC $_{50}$ indexes after 72 hour of drug incubation with Trabectedin/ Zalypsis/ Lurbinectedin

\begin{tabular}{cccc}
\hline & & Drug (pM) \\
\hline Cell Line & Trabectedin & Zalypsis & Lurbinectedin \\
A673 & $171.4 \pm 3.3$ & $95 \pm 6.02$ & $156 \pm 6.8$ \\
RM82 & $93.2 \pm 46.7$ & $138 \pm 17.8$ & $89 \pm 9.2$ \\
SK-ES-1 & $81.6 \pm 32.09$ & $82 \pm 8.1$ & $108 \pm 7,7$ \\
ES-CADO & $151.6 \pm 88.05$ & $127 \pm 11.2$ & $67.4 \pm 5.7$ \\
TC71 & $66.7 \pm 11.65$ & $166 \pm 10.2$ & $171 \pm 14.8$ \\
SJRH & $206.8 \pm 98.00$ & $88 \pm 10.1$ & $123 \pm 10.9$ \\
WE68 & $140.4 \pm 82.52$ & $228 \pm 13.8$ & $193 \pm 10.3$ \\
A4573 & $49.07 \pm 41.8$ & $186 \pm 12.2$ & $182 \pm 10.5$ \\
RDES & $50.2 \pm 30.29$ & $89 \pm 8.9$ & $121 \pm 7.3$ \\
STAET 1 & $106.0 \pm 44.84$ & $168 \pm 13.8$ & $190 \pm 8.5$ \\
SKNMC & $86.9 \pm 23.77$ & $94 \pm 8.6$ & $69 \pm 6.2$ \\
STAET 2.1 & $74.9 \pm 49.18$ & $110 \pm 9.6$ & $109 \pm 9.9$ \\
\hline
\end{tabular}




\subsection{Trabectedin derived compounds induced cytotoxicity in ES cells}

According to our data, Zalypsis and Lurbinectedin were also highly active against ES cells in the sub-nanomolar range. We observed that, in terms of proliferation inhibition, ES cells were slightly more sensitive to Trabectedin than Zalypsis and Lurbinectedin. The median of $\mathrm{IC}_{50}$ of proliferation was of $118 \pm 10.1$ pM for Zalypsis and 123 \pm 8.8 pM for Lurbinectedin (Table XVIII).

Later, we studied the effects of Trabectedin analogues on apoptosis. Firstly, we evaluated the concomitant activity of caspases 3 and 7 in two ES cell lines, TC71 and A4573. We observed that dosages of 500 pM of Zalypsis and Lurbinectedin were able to induce similar levels of apoptosis to Trabectedin in the A4573 cell line, the most sensitive cell line to Trabectedin (Figure 27A).

A4573

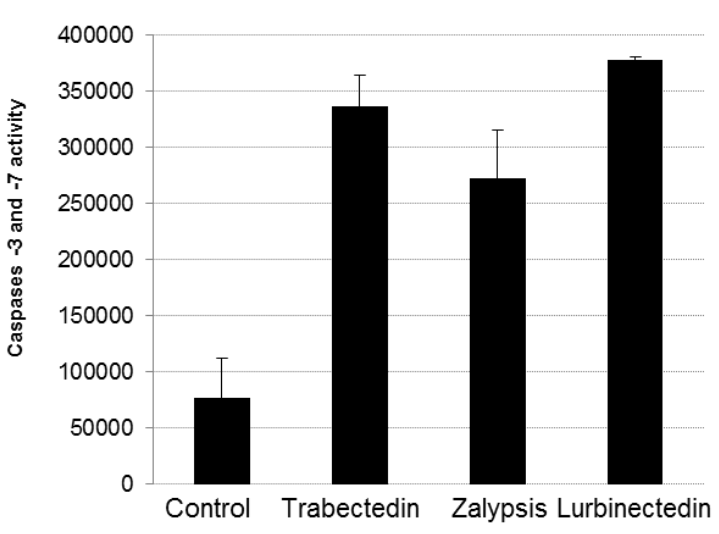

TC71

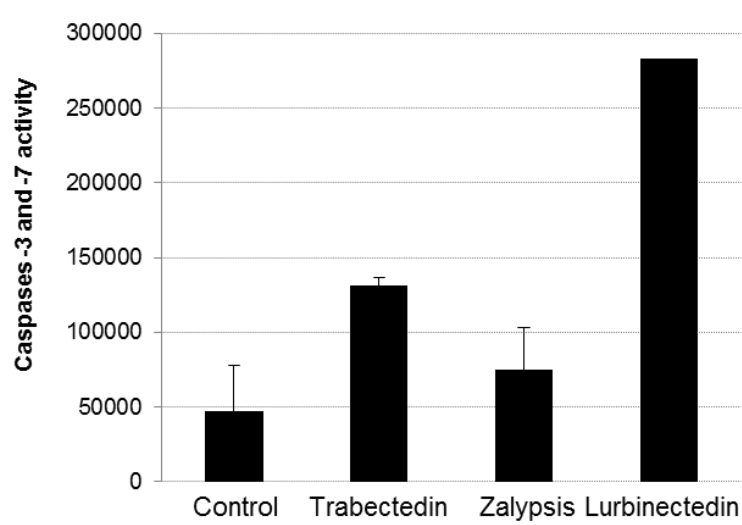

Figure 27. Caspases -3 and -7 activity after 24 hour of drug incubation in two ES cell lines. All drugs induced a significant increase of caspases activity when compared to the untreated condition. This was more obvious in the A4573 compared to the TC71 cell line.

However the TC71 cell line presented less apoptosis after treatment with Trabectedin and Zalypsis than with Lurbinectedin. 
Later, we studied the effect of this same dosage (500 pM) of each drug in a larger set of ES cell lines and analyzed the levels of apoptosis/necrosis by FACS. Herein, we observed that in most cell lines, with the exception of WE68, Zalypsis and Lurbinectedin induced more apoptosis than Trabectedin itself (Table XIX.

Table XIX. Apoptosis /Necrosis induction after treatment with 500pM of Trabectedin/Zalypsis/Lurbinectedin

\begin{tabular}{cccc}
\hline & \multicolumn{3}{c}{ Apoptotic/Necrotic } \\
\hline Cell Line & Trabectedin & Zalypsis & Lurbinectedin \\
A673 & $51.29 \pm 6.2$ & $88.7 \pm 5.25$ & $82.5 \pm 12.98$ \\
RM82 & $54 \pm 11.53$ & $80.4 \pm 18$ & $60.62 \pm 24.5$ \\
SK-ES-1 & $53.44 \pm 9.9$ & $80.7 \pm 13.0$ & $77.3 \pm 7.9$ \\
CADO-ES & $57.8 \pm 5.8$ & $86.46 \pm 4.35$ & $96.76 \pm 1.04$ \\
TC71 & $70.4 \pm 8.5$ & $88.4 \pm 25.9$ & $86.08 \pm 24$ \\
SJRH & $59.7 \pm 6.7$ & $70.8 \pm 11.8$ & $84.9 \pm 8.17$ \\
WE68 & $64.87 \pm 10.56$ & $62.3 \pm 26.2$ & $50.3 \pm 23.7$ \\
A4573 & $81.82 \pm 3.2$ & $86.54 \pm 12.63$ & $94.4 \pm 4.47$ \\
RD-ES & $66.37 \pm 1.5$ & $88.5 \pm 13.3$ & $88.1 \pm 3.52$ \\
STAET 1 & $43.61 \pm 4.74$ & $97.51 \pm 2.96$ & $78.51 \pm 2.13$ \\
SKNMC & $88.2 \pm 11.25$ & $94.8 \pm 5.74$ & $91.85 \pm 9.8$ \\
\hline of apoptotic /necrotic cells \pm sd & &
\end{tabular}

Therefore, we found that in terms of apoptosis induction, these two drugs were more effective than Trabectedin. Furthermore, treatment with Zalypsis and Lurbinectedin also appeared to decrease the ability to invade when compared to the untreated condition and Trabectedin (Figure 28). 


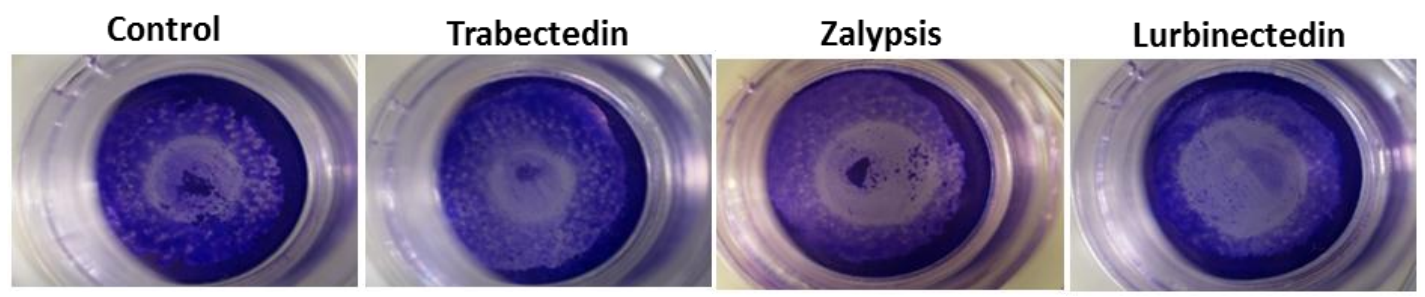

Figure 28. Migration Assays on the TC71 cell line after 72 hour of drug incubation. Zalypsis and Lurbinectedin, especially, reduced the ability to degrade the membrane and relocate onto the bottom well, when compared to Trabectedin and the untreated condition

Additionally, after 24 hours of exposure to $200 \mathrm{pM}$ of each drug separately, we observed cell cycle arrest with all the agents in the most sensitive cell line, A4573. A clear S-phase accumulation was observed after treatment with Trabectedin/Zalypsis/Lurbinectedin with reduction of the G0/G1 phase and the G2/M phase (Figure 29).

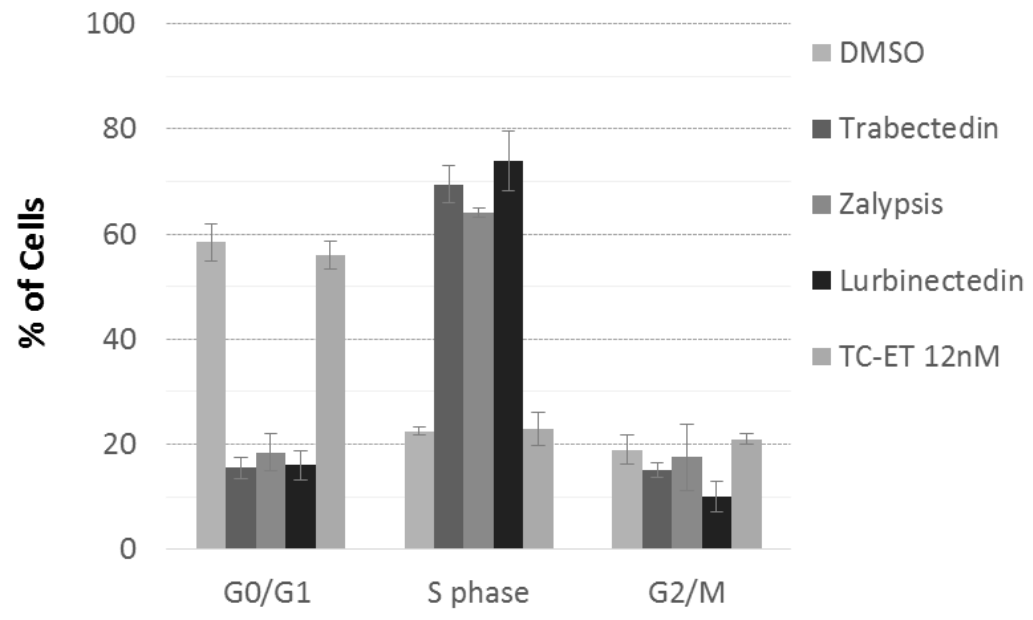

Figure 29. Cell cycle profiling on the A4573 cell line after 24 hours of drug incubation. The TC-ET resistant cell line was used as a control. The three agents induce S-phase arrest when compared to the untreated condition. The resistant cell line presents a normal cell cycle profile. 


\subsection{Zalypsis and Lurbinectedin are unable to overcome resistance to}

\section{Trabectedin}

The TC-ET Trabectedin resistant cell line was developed to grow under high concentrations (12nM) of Trabectedin. [47] We assessed the effects of Trabectedin related compounds in this particular cell line. Initially we observed that treatment with increasing dosages of the related compounds, from $100 \mathrm{pM}$ to 500 pM showed no significant effects in cell proliferation (Figure 30A). In fact, treatment with $12 \mathrm{nM}$ of any of these drugs showed no significant decrease of TC-ET proliferation when compared to $12 \mathrm{nM}$ Trabectedin (Figure 30B). Determination of the IC50 of proliferation proved that these cells are also much more resistant to Zalypsis/ Lurbinectedin than to the parental cell line (Table XX).
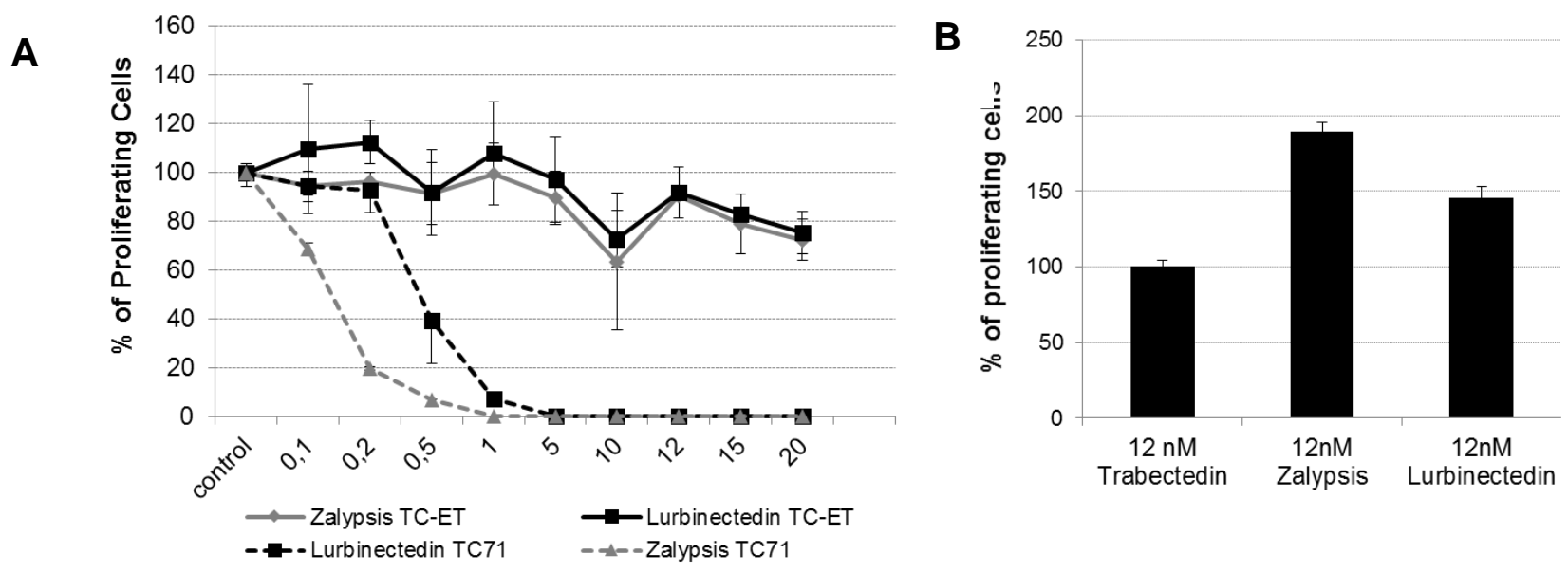

Figure 30. The cell line resistant to Trabectedin is also resistant to Zalypsis and Lurbinectedin. (A)Treatment with Zalypsis/Lurbinectedin did not affect the proliferation of TC-ET resistant cell line to Trabectedin, in contrast to the parental cell line, TC71. (B)Evaluation of the percentage of viable cells from the TC-ET resistant cell line after 72 hours incubation with $12 \mathrm{nM}$ of each agent. The cell line resistant to Trabectedin showed no growth impairment when Trabectedin maintenance concentration was substituted by the related compounds. 
Table XX. IC 50 Indexes of proliferation on the TC-ET $12 \mathrm{nM}$ compared to TC71 to Zalypsis and Lurbinectedin

\begin{tabular}{ccc}
\hline IC $_{50}$ & TC/ET 12nM & TC 71 \\
\hline Trabectedin & $19320 \pm 3740^{*}$ & $66.7 \pm 11.65$ \\
Zalypsis & $103356 \pm 26417$ & $122.25 \pm 5.5$ \\
Lurbinectedin & $41775 \pm 7384$ & $184.18 \pm 6.6$ \\
\hline
\end{tabular}

\subsection{Trabectedin, Zalypsis and Lurbinectedin induce DSB in ES cells}

DNA damage through DSB induction followed by its inaccurate repair is the most lethal type of cellular damage. The phosphorylation of H2AX at serine139 by DNA-PKs, during DNA fragmentation, displays $\gamma \mathrm{H} 2 \mathrm{AX}$ as one of the most commonly used markers of DSB. Also 53BP1 is a marker of DNA damage. This protein, responsible for binding p53 and DNA repair activation, appears to colocalize with $\mathrm{yH} 2 \mathrm{AX}$ in the presence of DSB.

Initially we studied whether Zalypsis and Lurbinectedin were also DSB inducers, through the formation of both $\mathrm{yH} 2 \mathrm{AX}$ intranuclear foci and/or overexpression of 53BP1 after treatment with lower dosages (200pM) for $24 \mathrm{~h}$ (Figure 31). All drugs induced $\mathrm{pH} 2 \mathrm{AX}$ foci in almost all cells. Most of these foci co-localized with 53BP1 foci, as usually in DSB induction. Also, intranuclear fragmentation assays showed that treatment with these agents induced DNA fragmentation. (Figure 31B) 
A

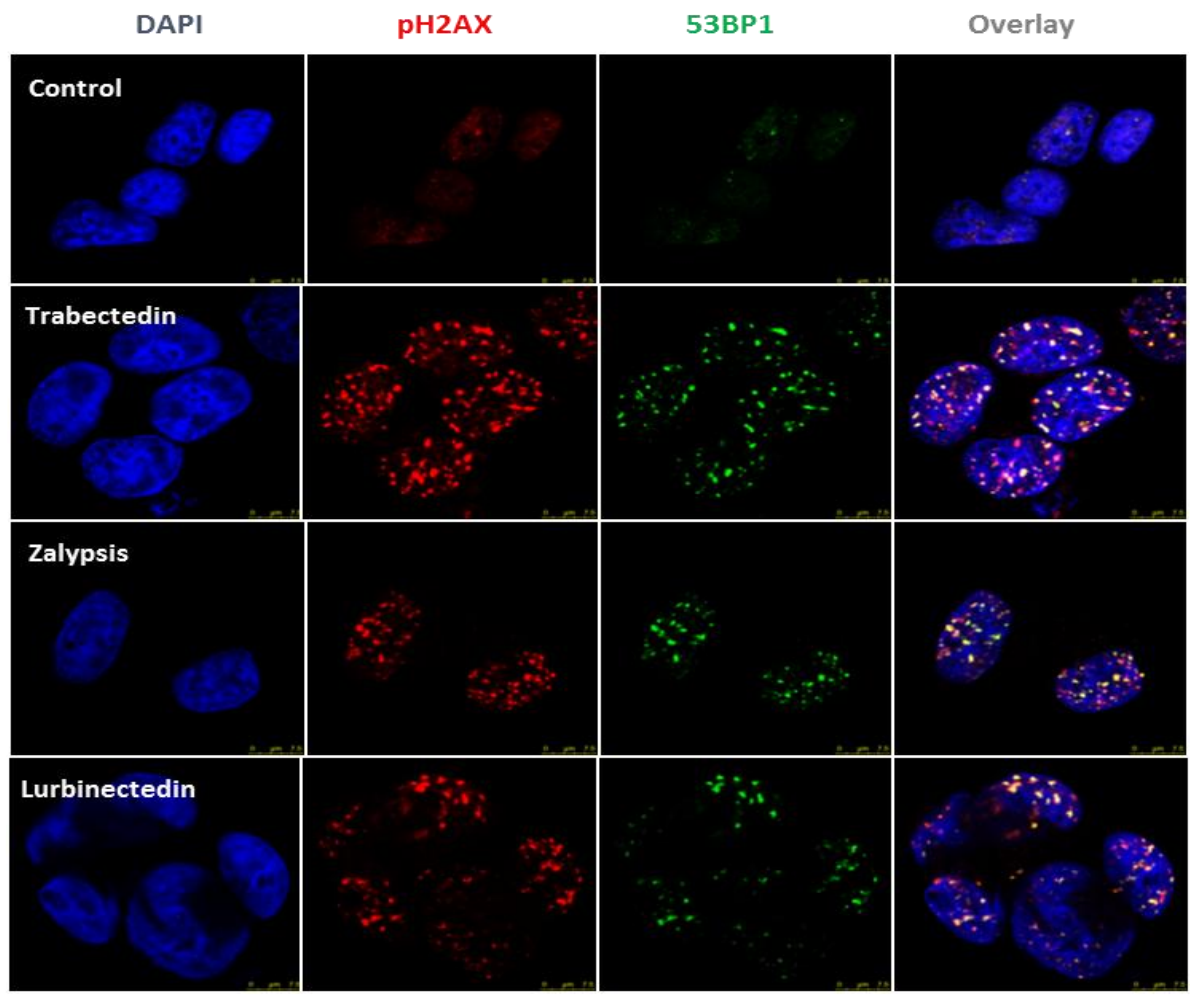

B

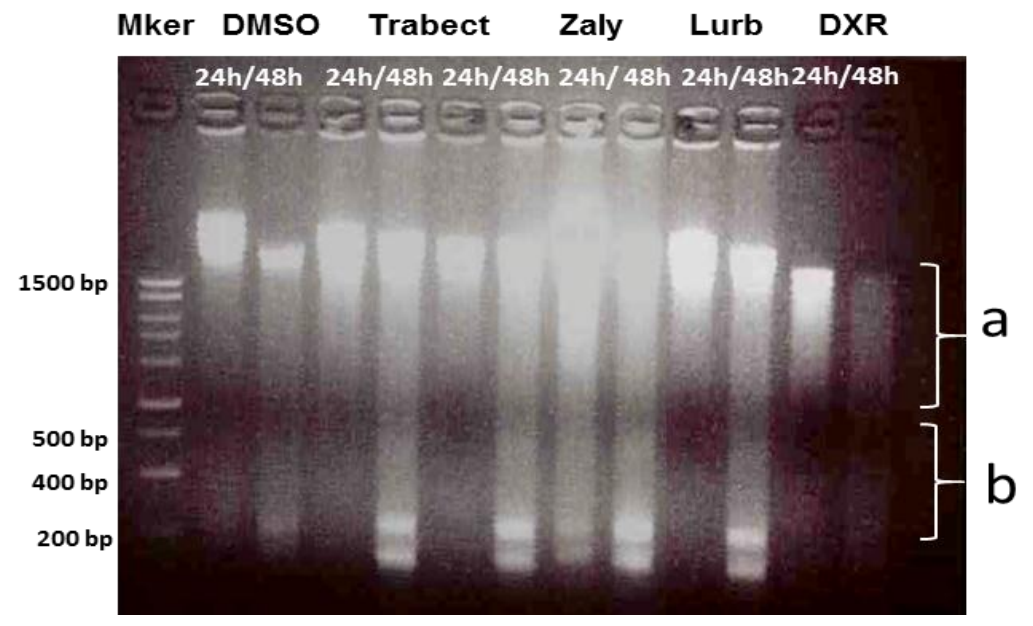

Figure 31. DNA Damage induction induced by treatment with Trabectedin/Zalypsis/Lurbinectedin. (A) DNA damage induction evaluated by the presence of $\mathrm{pH}_{2} \mathrm{AX}$ intranuclear foci (second column) and 53BP1 staining (third column), after treatment with 200pM of each agent for 8 hours. All agents induced DNA damage by the formation of DSB. (B) Evaluation of DNA fragmentation after treatment with $500 \mathrm{pM}$ of each drug for $24 / 48$ hours. Compared to the control, these agents clearly induce DNA fragmentation especially observed after 48 hours of drug incubation. (a represents DNA fragmentation by DSB induction and $b$ represents DNA fragmentation by apoptosis) 
Fragmentation by apoptosis (represented by b) is observed especially after $48 \mathrm{~h}$ of treatment, however, fragmentation by DSB induction could be observed even after $24 \mathrm{~h}$ treatment (represented by a). Moreover, we were interested in determining whether ES cells were able to repair the damage induced by these agents after drug washout. Cells were exposed to $8 \mathrm{~h}$ of treatment followed by drug washout and 16h of growth in medium without drug. Interestingly, we observed that cells maintained DSB accumulation as observed by the presence of persistent foci of $\mathrm{yH} 2 \mathrm{AX}$ (Figure 32).

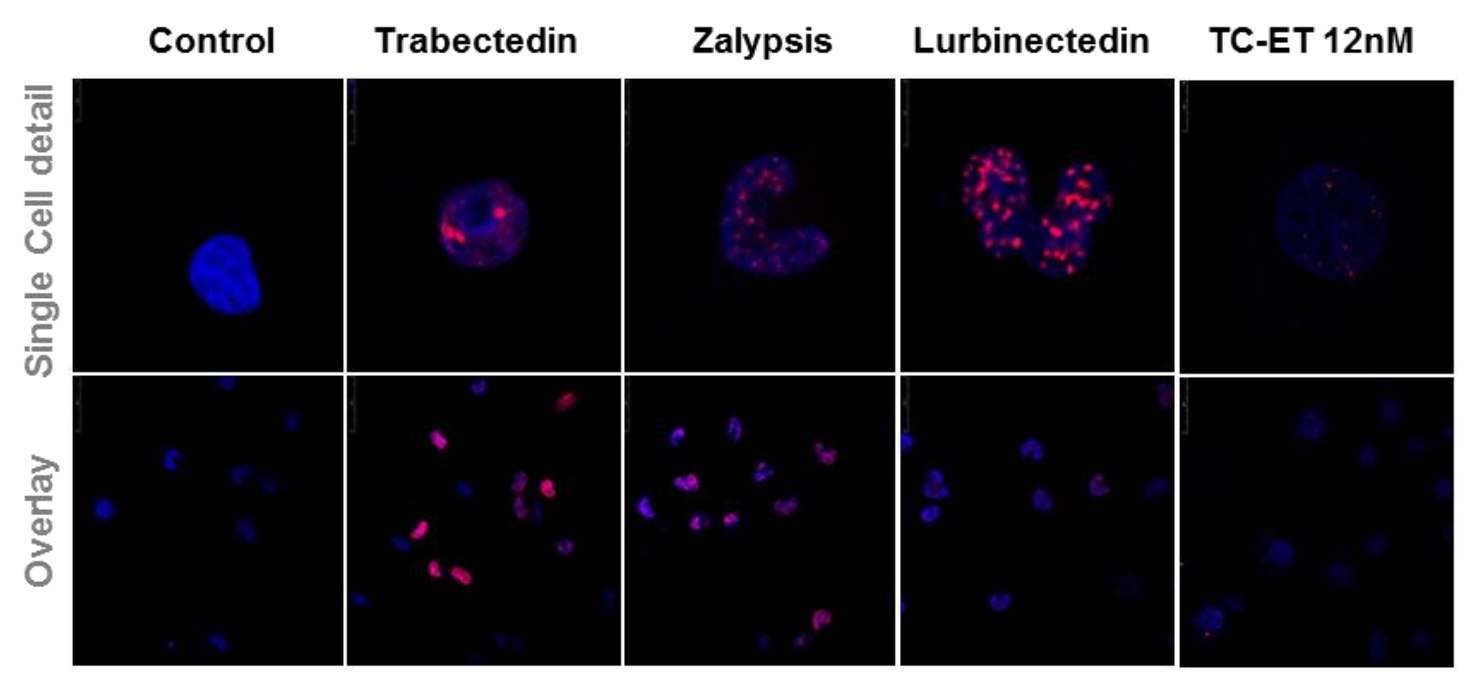

Figure 32. Induction of DSB after drug washout. DSB were persistent. DNA damage induction after 8 hours of drug incubation followed by 16 hours of drug washout. Here, we observe that when the drug was removed and cells were allowed to repair the induced damaged in medium without drug and with FBS, the lesions previously induced were maintained. Cells were enabled to repair the induced DSB, and the presence of $\mathrm{pH} 2 \mathrm{Ax}$ is observed in all cases. Regarding the TC-ET $12 \mathrm{nM}$ cell line, we observed that most cells didn't present foci of H2AX.

\subsection{Trabectedin, Zalypsis and Lurbinectedin, induce activation of DDR}

\section{pathways}

LDMA arrays to study the DNA damage and repair pathways activation (Qiagen) were performed in TC71 cell line after 16h treatment with the three 
compounds. Here we studied DNA damage induction with 200 pM or 500 pM of each drug.

Table XXI. Description of the up-regulated genes after 18 hours of treatment of the TC71 cell line.

A

\begin{tabular}{cc|cc|cc}
\hline \multicolumn{5}{c}{ Up-Regulated } \\
\hline Trabectedin & Exp value & Zalypsis & Exp value & Lurbinectedin & Exp value \\
\hline MSH4 & 1.381 & PMS1 & 1.082 & TOP3B & 1.345 \\
RAD23A & 1.149 & MSH4 & 1.076 & TOP3A & 1.314 \\
PMS1 & 1.142 & RPA3 & 1.041 & XRCC1 & 1.292 \\
XRCC1 & 1.129 & XPA & 1.025 & PMS1 & 1.128 \\
RPA3 & 1.095 & & & SMUG1 & 1.126 \\
BRCA1 & 1.081 & & & RAD23A & 1.110 \\
BRCA2 & 1.070 & & & BRCA1 & 1.110 \\
RAD18 & 1.066 & & & RPA3 & 1.055 \\
RAD50 & 1.062 & & & RAD54L & 1.049 \\
XRCC6BP1 & 1.062 & & & LIG3 & 1.045 \\
\hline (A)Up-regulated & genes & after & treatment & with 200 & pM
\end{tabular}

Trabectedin/Zalypsis/Lurbinectedin

B

\begin{tabular}{cc|cc|cc}
\hline \multicolumn{5}{c}{ Up-Regulated } \\
\hline Trabectedin & Exp value & Zalypsis & Exp value & Lurbinectedin & Exp value \\
\hline BRCA1 & 2.055 & UNG & 1.171 & RAD54L & 1.288 \\
RAD54L & 2.027 & RAD54L & 1.126 & EX01 & 1.133 \\
NEIL1 & 1.952 & RAD51C & 1.109 & MSH2 & 1.111 \\
BRCA2 & 1.778 & & & RAD51D & 1.103 \\
RFC1 & 1.766 & & & XRCC1 & 1.101 \\
POLD3 & 1.754 & & & DDB2 & 1.054 \\
ATXN3 & 1.672 & & & BRIP1 & 1.052 \\
BRIP1 & 1.663 & & & UNG & 1.042 \\
XRCC1 & 1.662 & & & POLB & 1.036 \\
XPC & 1.652 & & & MUTYH & 1.033 \\
\hline (B) Up-regulated genes after treatment & with 500 & pM
\end{tabular}

Trabectedin/Zalypsis/Lurbinectedin. 
In our first analysis, treatment with 200 pM of Trabectedin or Lurbinectedin, revealed the up-regulation of genes related to HR. (Fold change, Appendix) Treatment with Zalypsis showed less up-regulation of these pathways (Table XXI).

As an example, RPA3 was up-regulated in all the conditions at 200pM. RPA3, replication protein 3, usually accumulates around the DSB foci in form of a heterotrimeric complex and is described as a sensor of DNA damage signaling molecule. For the same time exposure, when cells were treated with $500 \mathrm{pM}$ of each agent (Table 4B), we observed an enrichment on HR related genes, namely BRCA1 and BRCA2 in the case of Trabectedin, EXO1 and RAD51D in the case of Lurbinectedin. Again, Zalypsis showed a distinct panel of up-regulated genes, in comparison to the other drugs, however HR related genes RAD54L and RAD51C were also up regulated. XPA, also appeared up-regulated in most cases (Table XXI).

Protein analysis after $24 \mathrm{~h}$ of treatment showed that treatment with $500 \mathrm{pM}$ increases the presence of cleaved PARP and cleaved caspase-3, markers of apoptosis. Over-expression of RAD52 HR effector protein, one of the proteins involved in the complex formed by RAD51 related molecules, was also observed after treatment with all drugs, in comparison to the control (untreated condition), especially at the highest concentrations. No differences on the expression of NER component ERCC1 were observed during treatment (Figure 33). 


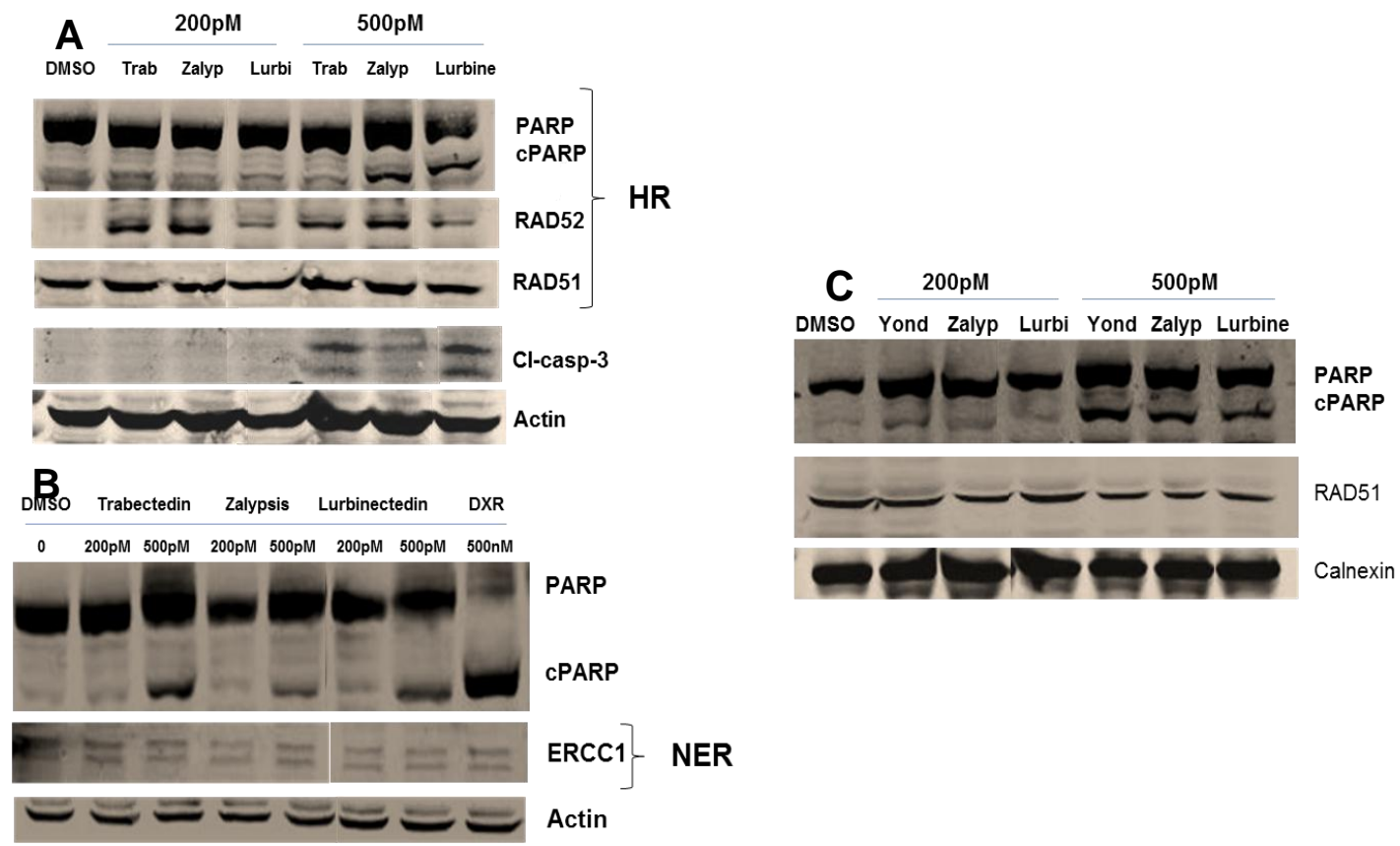

Figure 33. Western Blotting analysis of DDR protein expression on two ES cell lines after treatment. (A) Evaluation of apoptosis (PARP/ cPARP; cleaved caspase-3) and DNA damage repair induction after treatment with 200pM/500pM of each drug in the TC71 cell line.(B) Cleavage of PARP and caspase-3 is especially observed after incubation for 24 hours with $500 \mathrm{pM}$ of each agent. In fact, RAD52, showed a clear increase after treatment. This is especially observed when cells were treated with Trabectedin, Zalypsis. On the contrary, no changes on RAD51 were observed. (C) On the lower panel there were no differences on ERCC1 expression while PARP was cleaved. On the right panel, now in the CADO cell line, PARP cleavage is observed after treatment, especially with $500 \mathrm{pM}$ dosages of 
The Pathogenesis of Ewing Sarcoma/ Results 
3.

Trabectedin efficacy in ES is greatly increased by combination with anti-IGF signaling agents

Combination of Linsitinib with Trabectedin 


\section{Abstract}

The goal of this study was to identify the mechanisms that limit the efficacy of Trabectedin (ET-743, Yondelis ${ }^{\mathrm{TM}}$ ) in $\mathrm{ES}$, to develop a clinically relevant combination therapy. By chromatin immunoprecipitation, we analyzed the EWSR1FLI1 binding to the promoters of several target genes such as TGF $\beta R 2$, CD99, insulin-like growth factor receptor 1 (IGF-1R), and IGF-1 either in vitro and in xenografts treated with Trabectedin or doxorubicin. Combined therapy with Trabectedin and anti-IGF-1R agents (AVE1642 HAb; OSI-906) was tested in vitro and in xenografts. We confirm that both Trabectedin and doxorubicin were able to strongly reduce the binding of EWSR1-FLI1 (both type 1 and type 2) to two target genes (TGFßR2 and CD99), either in vitro and in xenografts. However, Trabectedin, but not doxorubicin, was also able to increase the occupancy of EWSR1-FLI1 to IGF-1R promoters, leading to IGF-1R upregulation. Inhibition of IGF-1R either by the specific AVE1642 HAb or by the dual IGF-1R/Insulin receptor inhibitor OSI-906 (Linsitinib) greatly potentiated the efficacy of Trabectedin in all 14 ES cell lines here considered, as well as in TC-71 and 6647 xenografts. Combined therapy induced synergistic cytotoxic effects. Trabectedin and OSI-906 deliver complementary messages that very likely converge on DNA damage response and repair pathways.

We showed that Trabectedin may not only inhibit but also enhance the binding of EWSR1-FLI1 to some target genes, leading to upregulation of IGF-1R. We provide the rationale for combining Trabectedin to anti-IGF-1R inhibitors.

This work was performed in collaboration with both Dr.Maurizio D'Incalci and Dr. Katia's Scotlandi's groups at the Rizzoli Institute in Bologna, Italy (3.1 and 3.2). 


\subsection{Trabectedin disrupts EWSR1-FLI1 binding to some DNA targets but increases recruitment to IGF-1R promoter either in in vitro or ex vivo models}

We used Chromatin Immunoprecipitation (ChIP) analysis to monitor the binding of the EWSR1-FLI1 chimera to some well-known target genes such as TGFßR2 and CD99, reported to be increased by EWSR1-FLI1 and proven to have a major role in ES aggressiveness [73], as well as IGF-1R and IGF-1 promoters. ChIP indicated that the amount of EWSR1-FLI1 chimera bound to the TGF $\beta$ R2 and CD99 promoters was significantly reduced after $1 \mathrm{~h}$ treatment with trabectedin both in TC-71 cells, which display EWSR1-FLI1 type1 chimera, and in the 6647 cell line, which displays EWSR1-FLI1 type2 hybrid, at pharmacological concentrations (Figure 34).
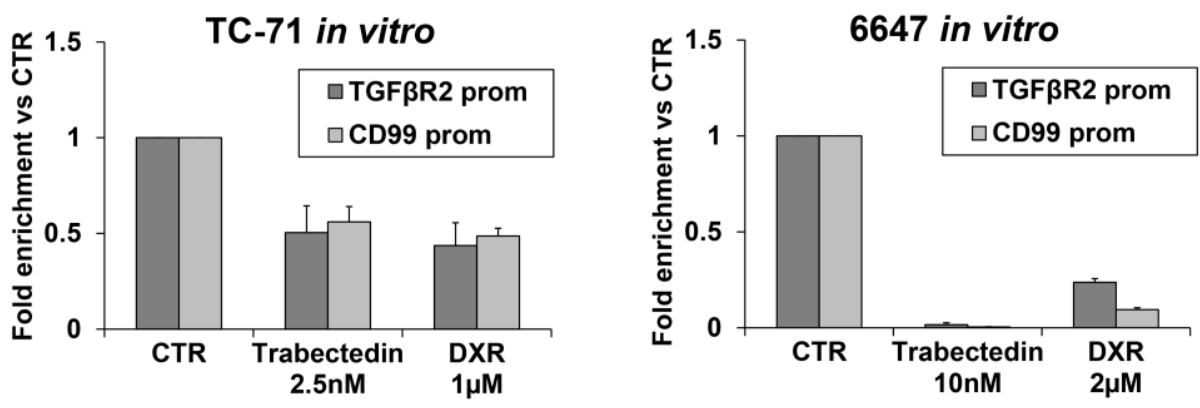

Figure 34. Trabectedin caused a dysregulation in the binding of EWS-FLI1 chimera to specific promoters. ChIP assays were carried out in vitro on TC-71 and 6647 ES cells, after treatments with Trabectedin or DXR. EWS-FLI1 was precipitated by the anti-FLI1 antibody. The decrease in the binding of the chimera EWS-FLI1 type 1 (TC-71) and type 2(6647) to CD99 and TGF $\beta$ R2 promoters was observed in vitro. Results were obtained by qPCR and are reported as fold enrichment over the control.

The binding of the chimera EWSR1-FLI1 type 1 (TC-71) and type 2 (6647) to the CD99 and TGF $\beta$ R2 promoters was evaluated also in mouse xenografts 
after IV administration of trabectedin $(0.15 \mathrm{mg} / \mathrm{kg}$, every seven days for three times, $q 7 \mathrm{dx} 3$ ) and DXR (8 $\mathrm{mg} / \mathrm{kg}$, every seven days for two times, $\mathrm{q} 7 \mathrm{dx} 2$ ). As shown in the TC-71 ES model, Trabectedin was more active (best T/C 56.2\% at day 20) than DXR (reference compound, best T/C 79.5\% at days 22) (Appendix). Instead in 6647 xenograft model, DXR was extremely effective (best T/C 14.3\% at days 21) while Trabectedin was less active (best $\mathrm{T} / \mathrm{C} 48.6 \%$ at days 21). (Appendix, Figure 60S) In both cases, however, Trabectedin and DXR were able to displace EWSR1-FLI1 chimera from CD99 and TGF $\beta$ R2 promoters, although with different kinetics (Figure 35).
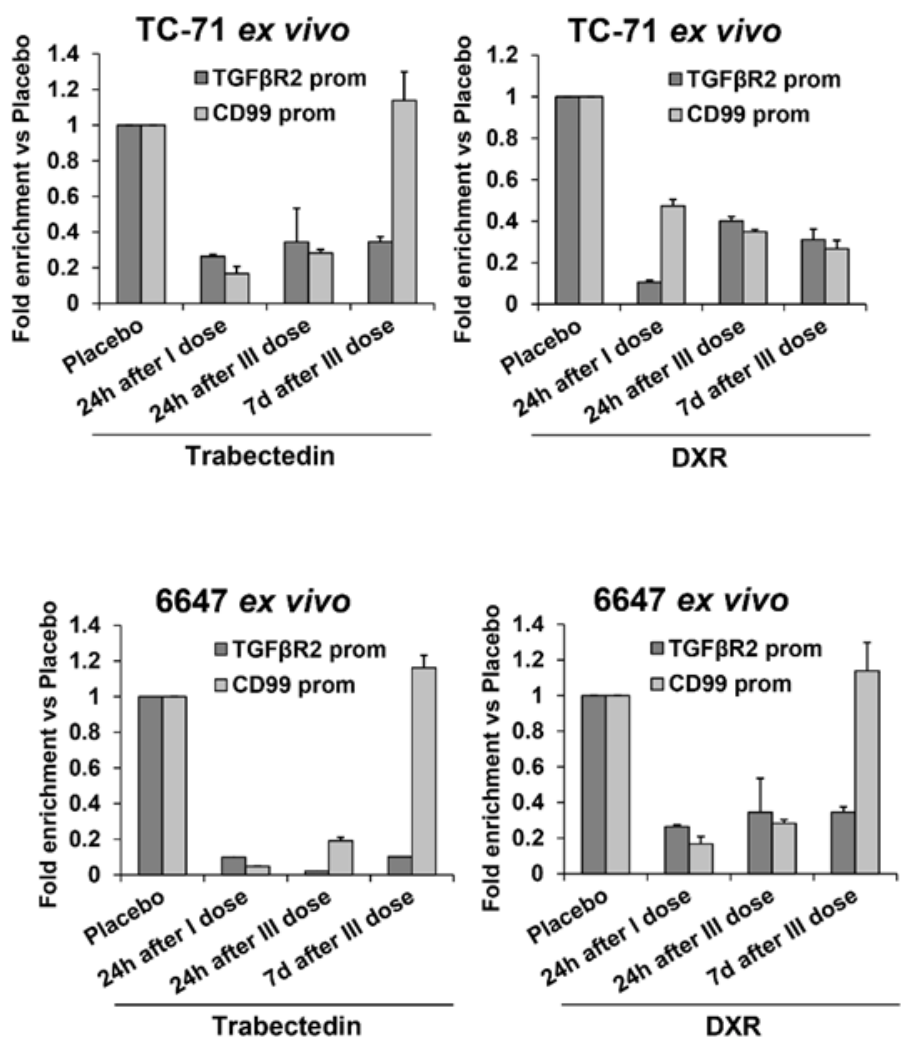

Figure 35. Trabectedin caused a dysregulation in the binding of EWS-FLI1 chimera to specific promoters. The decrease in the binding of the chimera EWSFLI1 type 1 (TC-71) and type 2(6647) to CD99 and TGF $\beta R 2$ promoters was also observed in vivo, in xenografts 
Trabectedin was able to cause the detachment of EWSR1-FLI1 chimera from both CD99 and TGF $\beta R 2$ promoters already $24 \mathrm{~h}$ after the first dose both in TC-71 and 6647 cells. The re-attachment was observed starting from seven days after the third treatment.

Besides inhibitory effects, Trabectedin (but not DXR) also caused an increase in the binding of the chimera to IGF-1R promoter in ES cells (Figure 36), while occupancy of the IGF-1 promoter appeared to be only slightly affected.

A
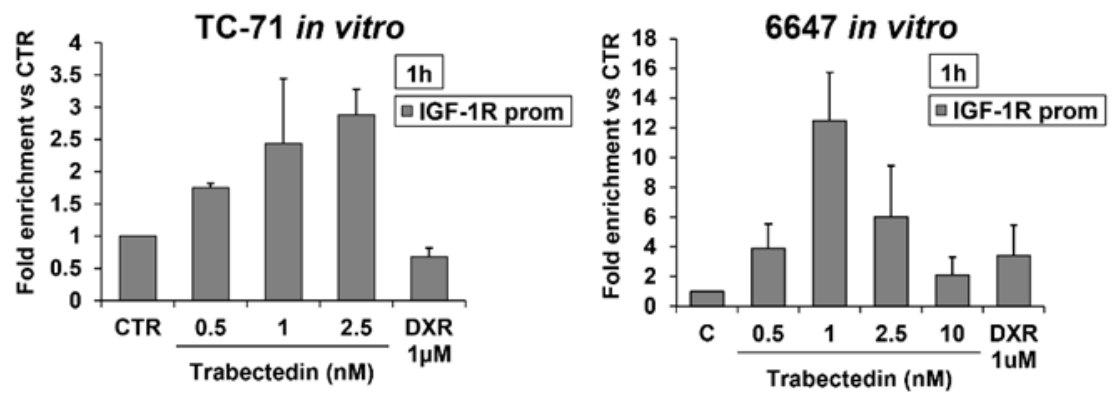

B

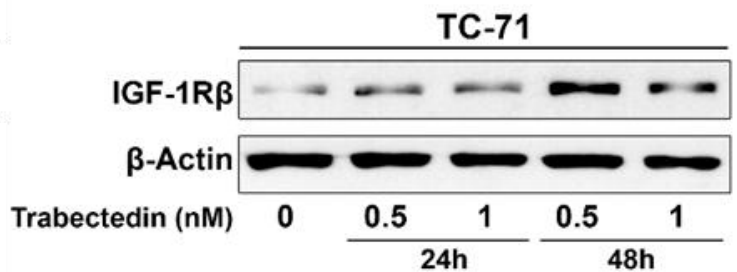

Figure 36. Increased recruitment of EWS-FLI1 on IGF-1R promoter in TC-71 and 6647 EWS cells treated for $1 \mathrm{~h}$ with Trabectedin or DXR. (A) A representative experiment is shown. Data represent recovery of each DNA fragment relative to total input DNA, respect to control. (B) Up-regulation of IGF-1R at protein level by western blotting after exposure to trabectedin, is represented on the bottom pannel

Enhancement of EWSR1-FLI1 occupancy to IGF-1R by Trabectedin was time-dependent, as shown either in vitro in TC-71 cells (or in vivo in TC-71 and 6647 xenografts (Figure 36A, 37). Up-regulation of the IGF-1R protein was also confirmed after $24 \mathrm{~h}$ of Trabectedin treatment (Figure 37B), in line with our 
previous data showing increased transcription and expression of IGF-1R in cells made resistant to Trabectedin [47] (Appendix, Figure 61S). These findings provided the rational for testing the combination of Trabectedin with anti-IGF-1R agents.
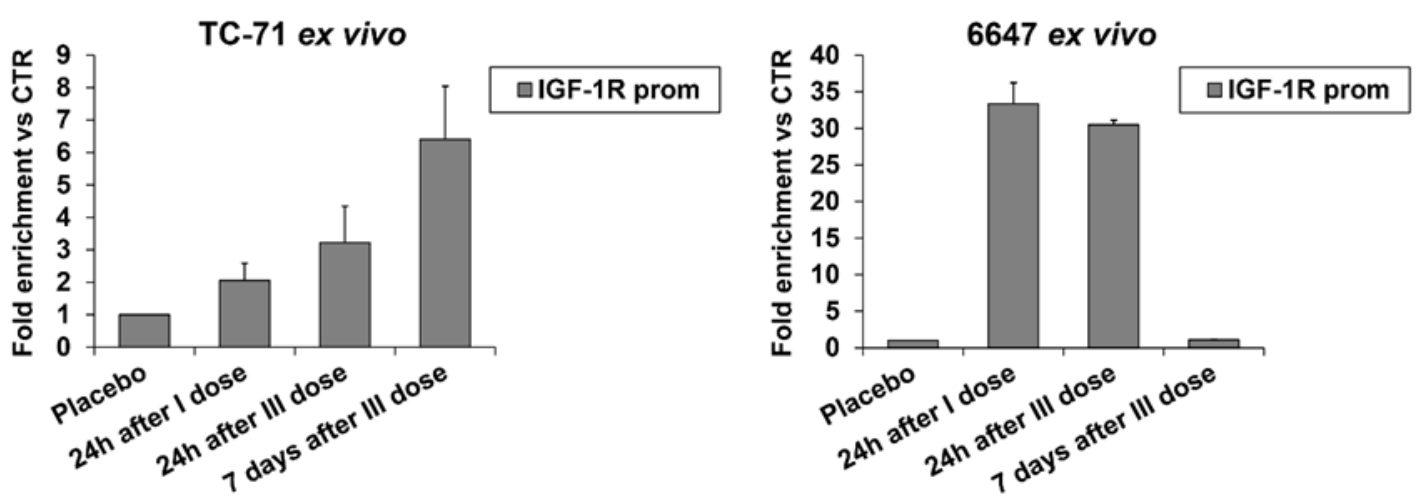

Figure 37. Time course of FLI1 association with the IGF-1R promoter in TC-71 and 6647 xenografts treated with trabectedin $(0.15 \mathrm{mg} / \mathrm{kg})$.

\subsection{Anti-tumor activity of the combination between Trabectedin and the anti IGF-1R HAb AVE1642}

The antitumor activity of Trabectedin alone or in combination with the antiIGF-1R AVE1642 HAb was evaluated in TC-71 xenograft model. The combination Trabectedin and AVE1642 HAb (best T/C 27.9 at days 20) showed a greater antitumor activity than Trabectedin (best T/C 40.3 at days 20) or AVE1642 HAb (best T/C 48.6 at days 15) used as single agents (Figure 38). 


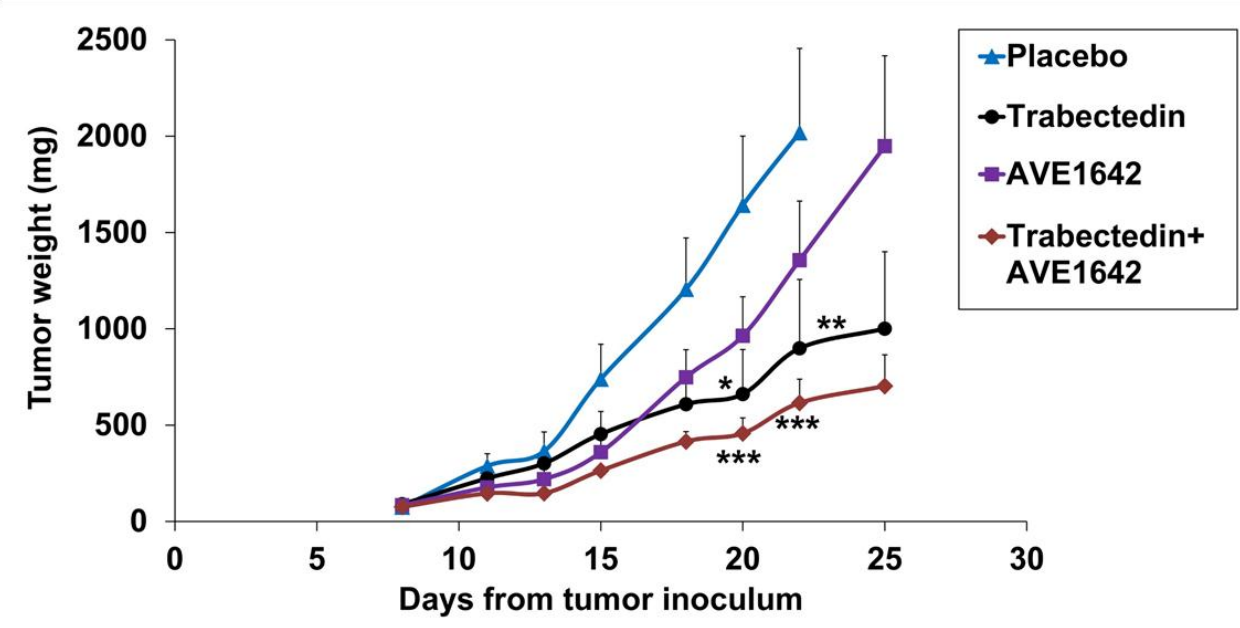

Figure 38. Efficacy of the combination of Trabectedin with the anti-IGF-1R HAb AVE1642 against TC-71 xenografts. The drugs were administered IV as follows: Trabectedin $0.15 \mathrm{mg} / \mathrm{kg}$, every seven days for three times (q7dx3) and AVE1642 $40 \mathrm{mg} / \mathrm{kg}$, and every three days for six times (q3dx6). ANOVA test: * $P$ $<0.05 ;{ }^{* *} \mathrm{P}<0.01 ;{ }^{* * *} \mathrm{P}<0.001$ compared with controls (Placebo). Points, tumour weight means; bars, SE.

Studies in myxoid liposarcoma indicate that Trabectedin besides inhibiting cell proliferation is also able to act as a differentiating agent by blocking the trans activating ability of the fusion gene product We could confirm the antiproliferative, pro-apoptotic and pro-differentiating activity of Trabectedin also in ES (Figure 39). Combination treatments with AVE1642 HAb did not influence the differentiation of TC-71 xenografts but further inhibited tumor cell proliferation (Figure 39) and increased apoptotic rate, supporting the combination of Trabectedin with anti-IGF-1R HAb against ES. 


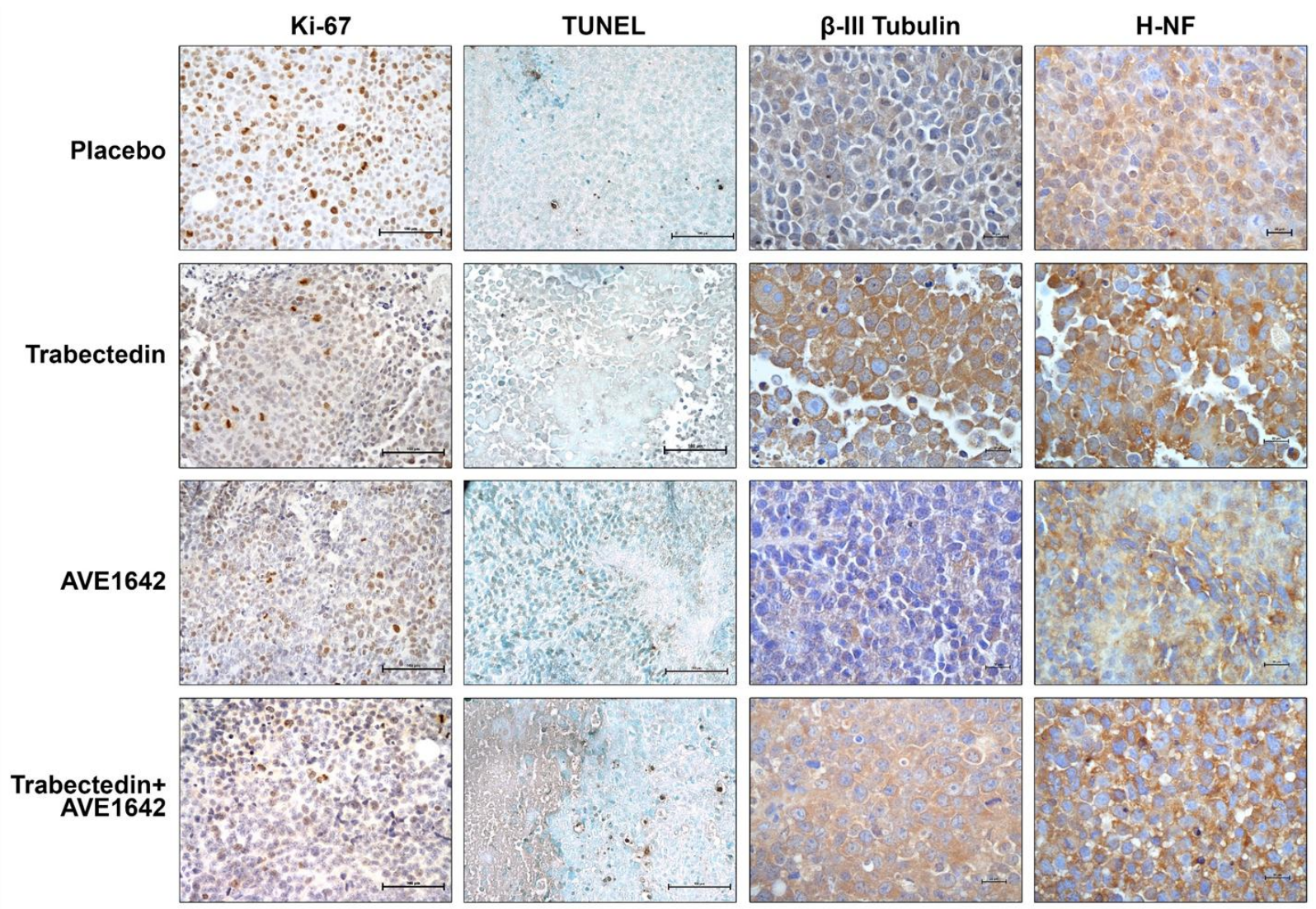

Figure 39. Representative immunohistochemical evaluation of Ki-67, apoptotic rate by TUNEL assay (magnification, x200) and neural differentiation markers ( $\beta$ III tubulin, H-NF magnification, x400), in untreated or treated tumors.

\subsection{Efficacy of the dual inhibitor anti-IGF-1R/IR OSI-906 in combination with}

\section{Trabectedin}

Considering that the great majority of ES express concomitant high levels of IR (33), which may overcome IGF-1R blockade, the efficacy of the dual inhibitor anti-IGF-1R/IR OSI-906 was evaluated in a panel of 14 ES cell lines (Table XII), including the TC/ET 12nM cell line, highly resistant to Trabectedin [47]. All the cell lines were highly sensitive to the inhibitor (Table XXII). 
Table XXII. Sensitivity of ES cell lines to the dual IGF1R/IR inhibitor OSI906. Expression level of IGF1R and/or IR does not correlate with OSI-906 efficacy

\begin{tabular}{|c|c|c|c|c|}
\hline Cell lines & $\begin{array}{l}\text { OSI-906 } \\
\text { (uM) } \pm \text { SE* }^{*}\end{array}$ & $\begin{array}{c}\text { IGF-IR } \\
\text { (ng/100ug of } \\
\text { proteins) }\end{array}$ & $\begin{array}{c}\text { IR } \\
\begin{array}{c}\text { (ng/100ug of } \\
\text { proteins) }\end{array}\end{array}$ & $\begin{array}{c}\text { Ratio } \\
\text { IGFIR:IR* }\end{array}$ \\
\hline TC-71 & $0.4 \pm 0.15$ & 2 & 0.32 & 6.25 \\
\hline WE-68 & $0.45 \pm 0.25$ & 4.9 & 0.8 & 6.125 \\
\hline SK-N-MC & $0.59 \pm 0.18$ & 3.69 & 2.87 & 1.28 \\
\hline LAP-35 & $0.17 \pm 0.07$ & 2.38 & 0.56 & 4.25 \\
\hline IOR/CAR & $1.402 \pm 0.66$ & 11.2 & 1.27 & 8.81 \\
\hline 6647 & $1.25 \pm 0.45$ & 0.81 & 4.13 & 0.19 \\
\hline RD-ES & $0.63 \pm 0.14$ & 3.74 & 2.39 & 1.56 \\
\hline SKES-1 & $0.76 \pm 0.15$ & 1.82 & 6.03 & 0.30 \\
\hline RM82 & $0.2 \pm 0.14$ & 6.31 & 4.94 & 1.27 \\
\hline CADO-ES & $3.77 \pm 0.47$ & 2.6 & 1 & 2.59 \\
\hline TTC466 & $0.39 \pm 0.13$ & 3.68 & 1.31 & 2.8 \\
\hline STAET 2.1 & $2.05 \pm 0.47$ & 6.27 & 2.30 & 2.73 \\
\hline $\begin{array}{c}\text { IOR/BRZ- } \\
2010\end{array}$ & $0.61 \pm 0.13$ & 28.15 & 2.74 & 10.27 \\
\hline TC/ET12nM & $0.1 \pm 0.009$ & ND & ND & ND \\
\hline
\end{tabular}

Sensitivity to OSI-906 was not affected by the relative expression of IGF$1 \mathrm{R}$ and/or IR ( $p=0.629$, Spearman's test), in keeping with its activity as a dual inhibitor. As expected, exposure of cells to OSI-906 400nM (IC50 values) inhibited both MAPK and AKT signaling, the two main IGF-1R/IR intracellular pathways (Figure 40). OSI-906 was found to induce a blockade of cells in G1 phase of cell cycle in TC-71 and SK-N-MC cells (Figure 41), which displayed either truncations (TC-71) or major deletions (SK-N-MC) of p53, whereas in the wild-type-p53 WE-68 cell line the dual IGF-1R/IR inhibition appeared to preferentially result in apoptotic effects (Figure 41). 
TC71

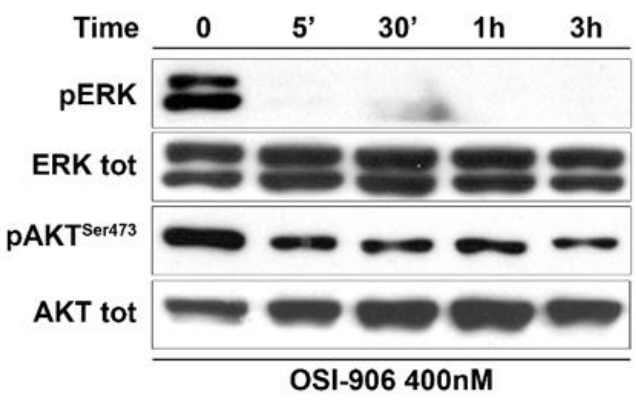

WE68

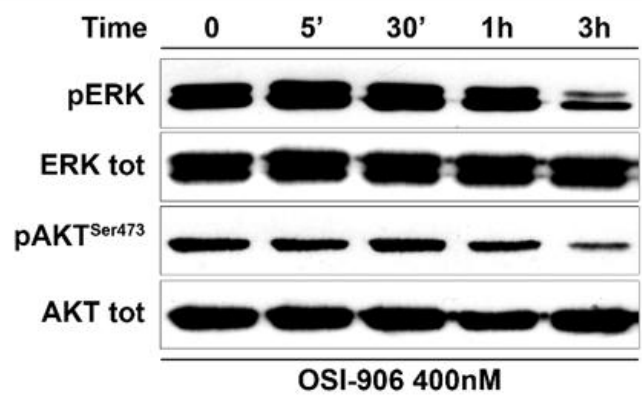

Figure 40. Efficacy of the dual inhibitor anti-IGF-1R/IR OSI-906 in combination with Trabectedin: Protein expression. Analysis of the principal mediators of IGF1R/IR signaling in TC-71 and WE-68 ES cell lines after treatments with OSI-906 by western blotting. Total proteins were used as controls. Blots are representative of two independent experiments

Table XXIII. Efficacy of combined treatments of Trabectedin with OSI-906 in EWS cell lines

\begin{tabular}{|c|c|c|}
\hline Cell lines & 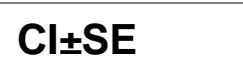 & Effects \\
\hline SKES-1 & $0.784 \pm 0.03$ & synergistic \\
\hline SK-N-MC & $0.789 \pm 0.08$ & synergistic \\
\hline LAP-35 & $0.734 \pm 0.08$ & synergistic \\
\hline 6647 & $0.687 \pm 0.06$ & synergistic \\
\hline RD-ES & $0.646 \pm 0.06$ & synergistic \\
\hline TC-71 & $0.819 \pm 0.01$ & synergistic \\
\hline TC/AVE1642 & $0.71 \pm 0.02$ & synergistic \\
\hline TC/AEW541 & $0.526 \pm 0.05$ & synergistic \\
\hline IOR/CAR & $0.789 \pm 0.03$ & synergistic \\
\hline WE-68 & $0.356 \pm 0.02$ & synergistic \\
\hline BRZ-2010 & $0.834 \pm 0.07$ & synergistic \\
\hline RM-82 & $0.6 \pm 0.09$ & synergistic \\
\hline CADO-ES & $0.77 \pm 0.98$ & synergistic \\
\hline
\end{tabular}


The combination of OSI-906 with Trabectedin gave synergistic effects in all ES cell lines, including the cell lines resistant to anti-IGF-1R agents (AVE1642 or AEW-541) or to Trabectedin (TC/ET 12 nM) (Table XXIII).

Interestingly, when OSI-906 was combined to Trabectedin we observed an improved apoptosis induction, both in p53wt and p53mutated cells (Figure 41).
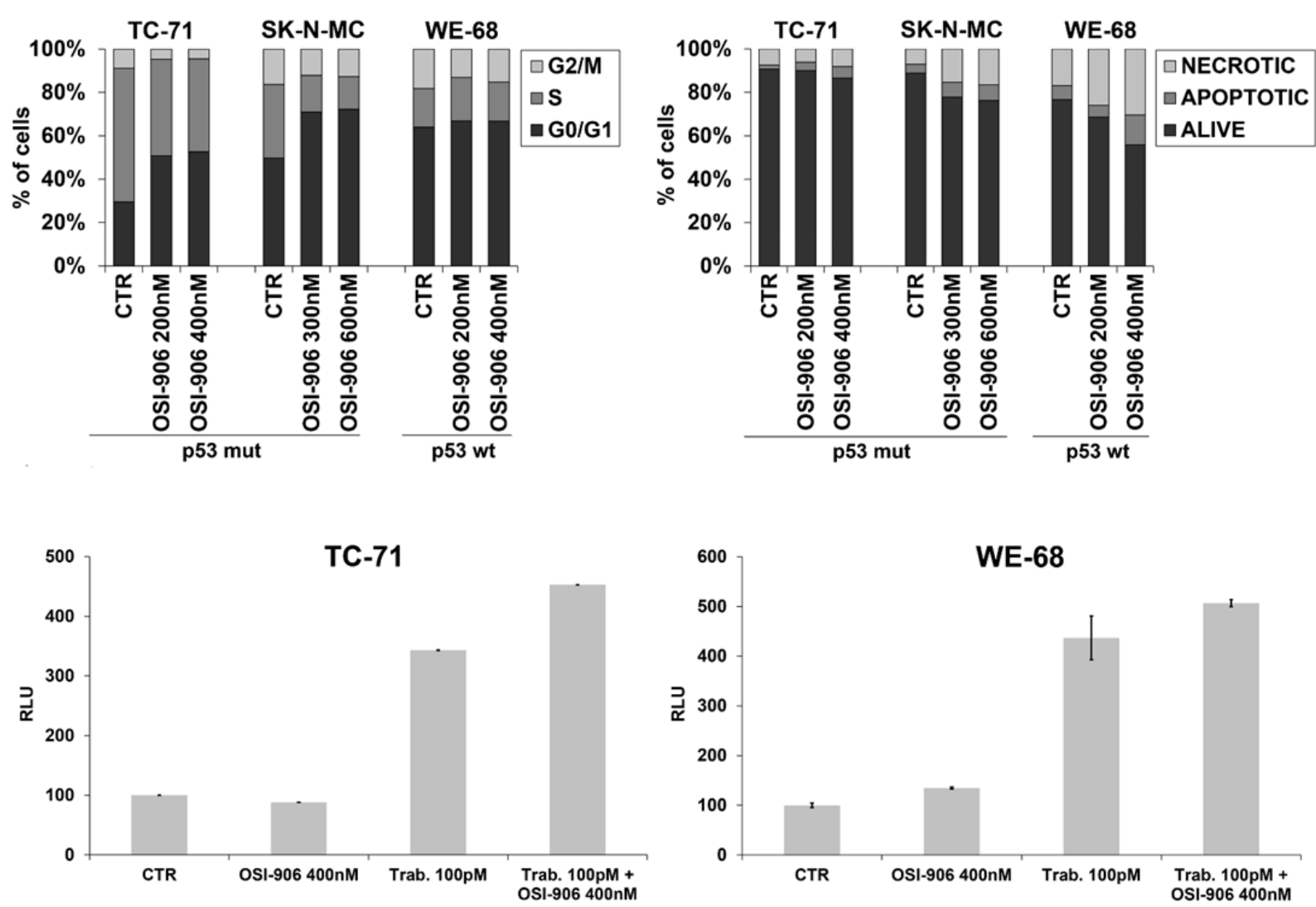

Figure 41. Efficacy of the dual inhibitor anti-IGF-1R/IR OSI-906 in combination with Trabectedin: Cell cycle profiling and apoptosis induction. Cell cycle evaluation of EWS cell lines after 24h exposure to OSI-906. Data are expressed as mean percentages of two independent experiments. OSI-906 increased percentage of G0/G1 cells in TC-71 and SK-N-MC, which carry p53 mutations, while no significant effects on cell cycle was observed in p53wt WE-68 cell line. , Annexin V-PI analysis, expressed as percentage of viable, apoptotic or necrotic cells, in EWS cells after 48h exposure to OSI-906. Values are representative of 3 independent experiments. Percentage of apoptotic or necrotic cells is remarkably higher in the p53wt WE-68 cell line respect to cells with loss-of-function p53 alterations. Caspase cleavage activation (RLU) in TC-71 and WE-68 treated with Trabectedin and/or OSI-906 for $48 \mathrm{~h}$. All treatments are normalized respect to control. Bars, mean of two independent experiments $\pm S E$. 
Thus, combination regimen resulted in cytotoxic, rather than cytostatic effects, independently of p53 status, further supporting its use in clinical trials. This advantageous pro-apoptotic cell death may derive from the combination of two different inputs. On the one hand, the inhibition of the IGF system may block the anti-apoptotic effects of IGF-1R/IR-A due to the disruption of AKT and/or 14.3.3/Raf-1/Nedd4 pathways [122, 123]; on the other hand Trabectedin acts as a DNA damaging agent inducing DSB [124]. Here, we studied DNA damage induction by the presence of $\mathrm{\gamma H} 2 \mathrm{AX}$ and 53BP1 intranuclear foci after $24 \mathrm{~h}$ treatment (Figure 42) showing that Trabectedin was a potent DSB inducer, in contrast to OSI-906, and that DNA damage was present when cells were treated with the combination of trabectedin and OSI-906.

DNA fragmentation assays showed that OSI-906 alone and particularly the combination with Trabectedin lead to DNA fragmentation (Figure 42B). The presence of DNA fragments ( $<500 \mathrm{bp})$ after cell exposure to OSI-906 is not surprising since these fragments are likely related to DNA degradation by apoptosis. Accordingly, OSI-906 favors the expression of apoptotic proteins such as CPARP, fraction $90 \mathrm{kDa}$ (Figure 43). PARP cleavage was also observed in TC-ET resistant cell line after treatment with increasing doses of OSI-906, thus confirming the complementary pro-apoptotic effects of the two drugs (Figure. 43). 
A

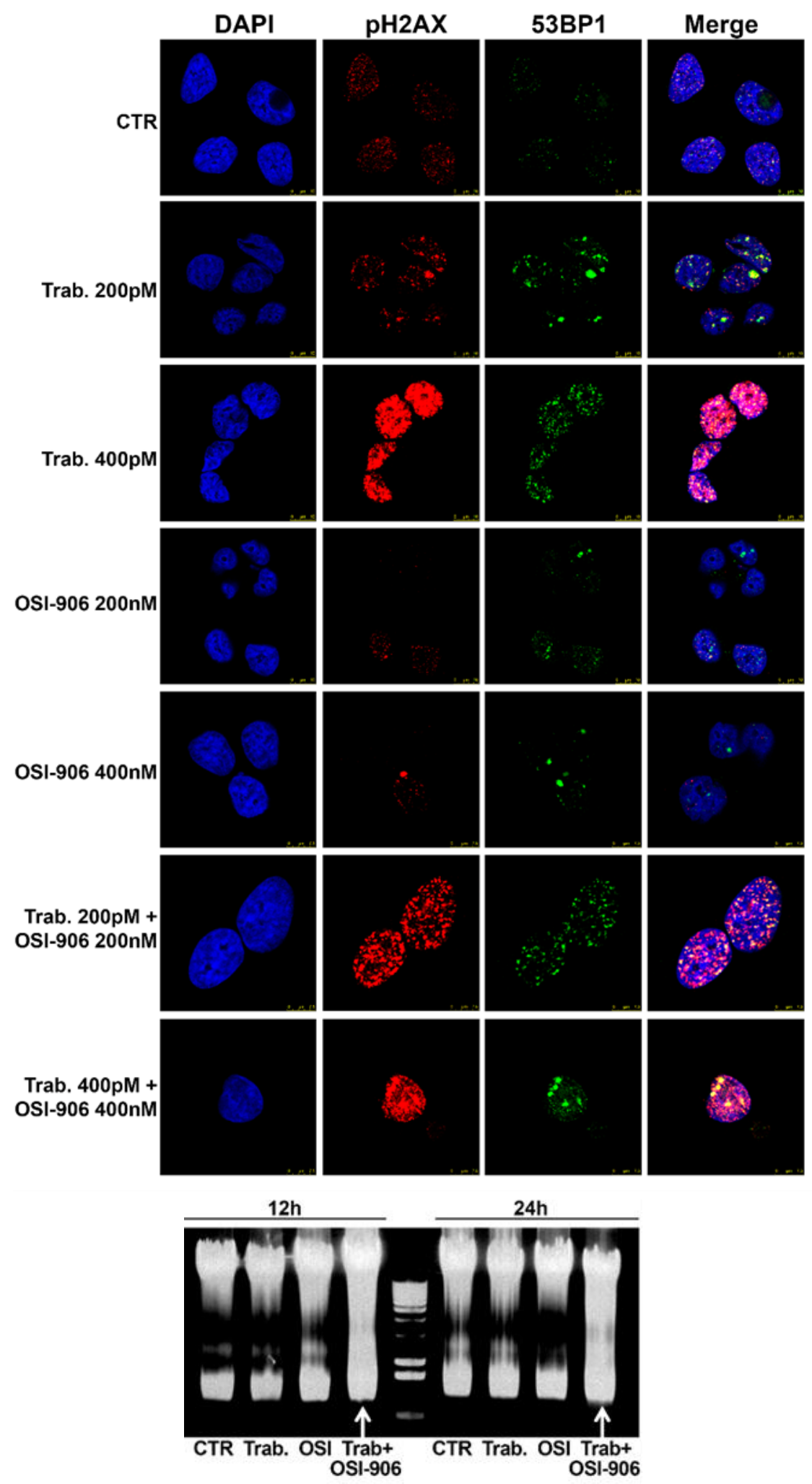

Figure 42. DNA damage induction after cell exposure to Trabectedin and/or OSI906. (A) Induction of H2AX phosphorylation ( $\mathrm{Y}-\mathrm{H} 2 \mathrm{AX}$ ) and 53BP1 expression in TC-71 cells after exposure to Trabectedin, and/or OSI-906 (200-400nM) for 24h (magnification $\times 400$ ). (B) DNA fragmentation induced in TC-71 cells after $12-24 \mathrm{~h}$ exposure to drugs. 
TC-71

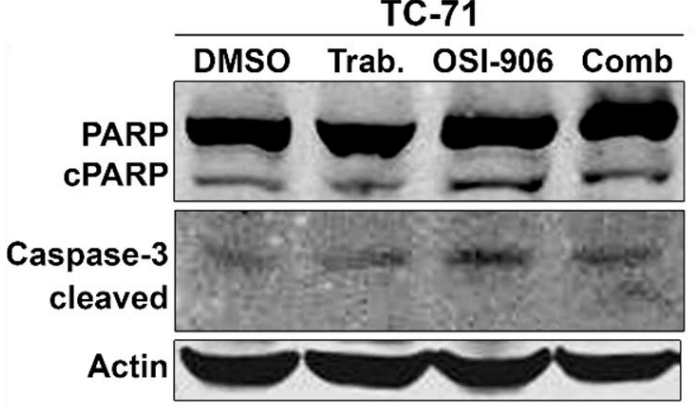

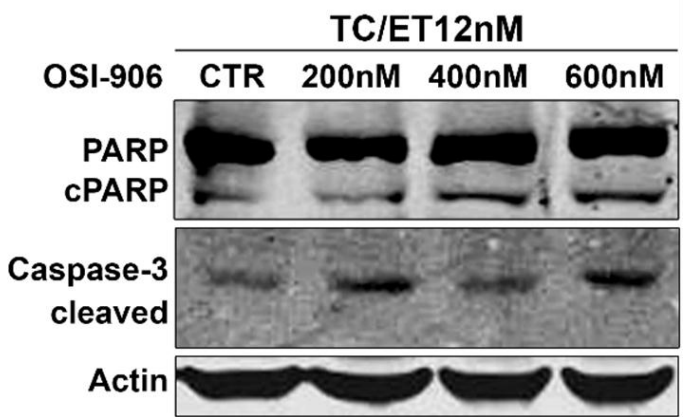

Figure 43. Evaluation of Caspase 3 and PARP cleavage by western blotting after $24 \mathrm{~h}$ exposure to drugs. Equal loading was monitored with beta-actin blotting.

To further characterize these effects, we analyzed the involvement of DNA repair pathways by using DNA damage low density arrays customized to cover HR, NER, BER and NHEJR pathways. Treatment with Trabectedin, but not with OSI-906, induced expression of BRCA1, BRCA2, key proteins in the HR pathway as well as XRCC1, member of SSR pathway (Figure 44). [101] The drug combination resulted in the up-regulation of members from HR pathway (RAD52, BRCA1 and BRCA2), NER proteins (XPA and ERCC1) and SSR pathways (XRCC1). Top associated Network functions affected by treatment with Trabectedin and/or OSI-906 were DNA replication and Cancer (score: 64); DNA Replication, Recombination, and Repair, Cellular Response to Therapeutics, Cell Cycle (score 28) and DNA Replication, Recombination, and Repair, Cellular Assembly and Organization, Cellular Function and Maintenance (score 26). 
A

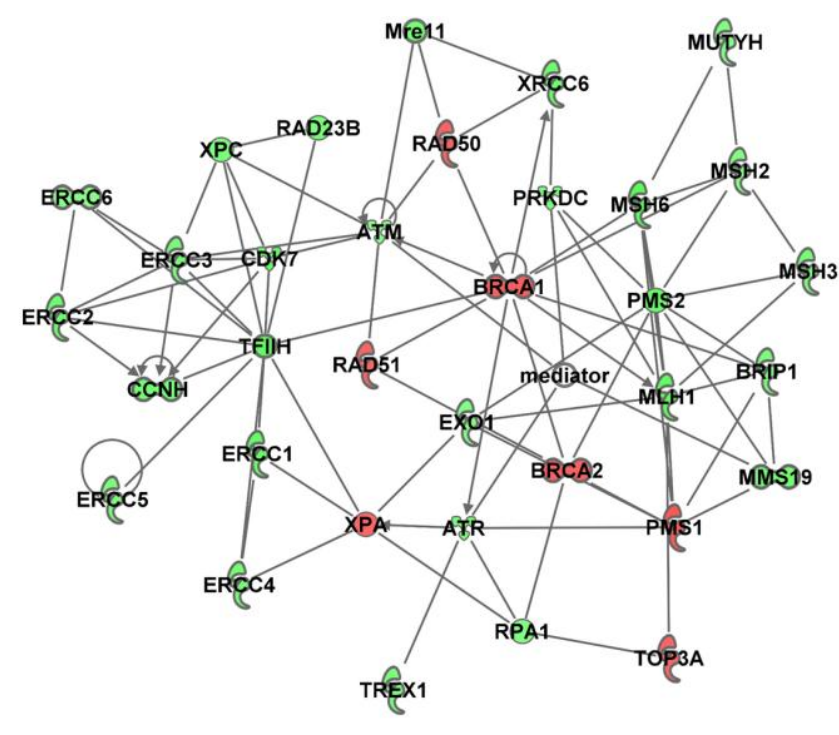

B

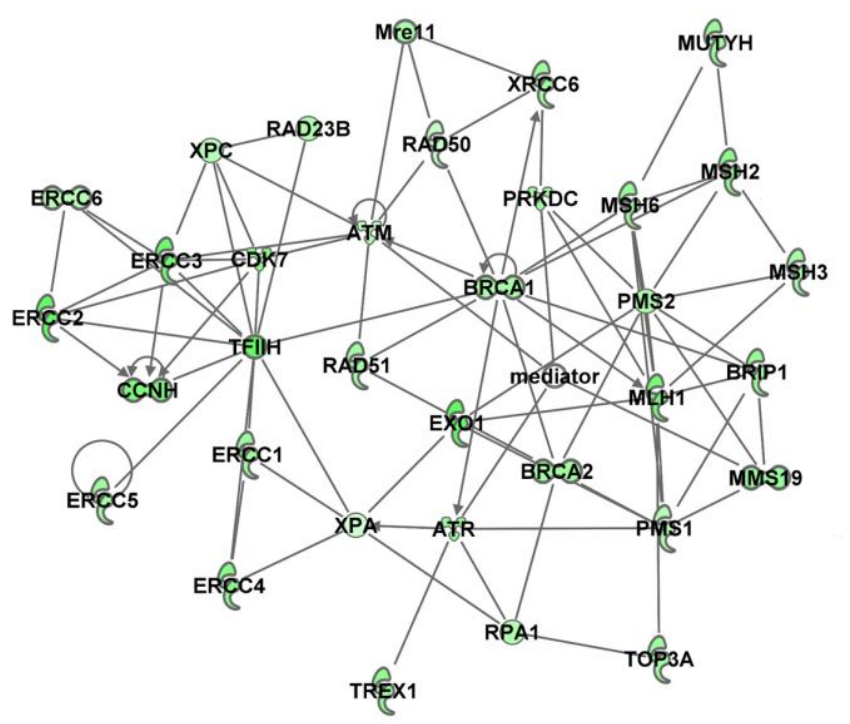

C

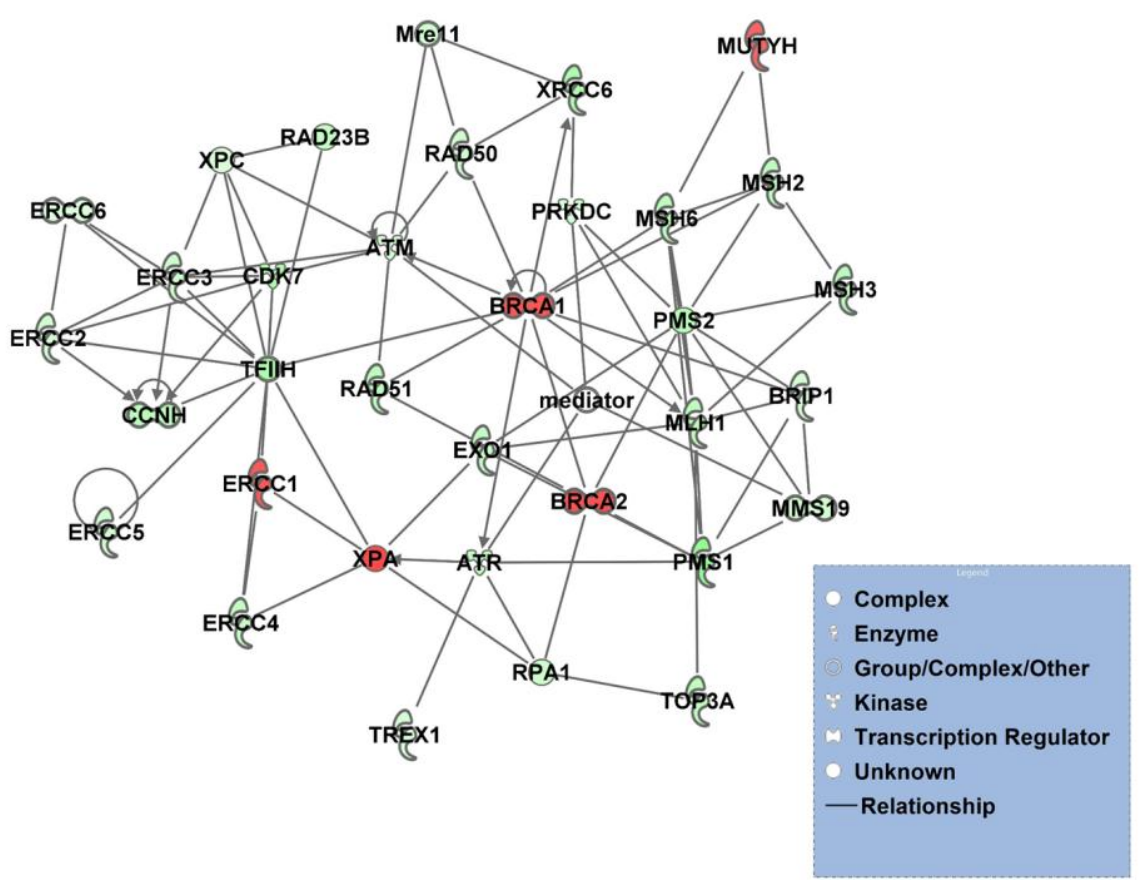

Figure 44. DNA damage repair pathways after treatment of TC-71 cells with Trabectedin and/or Osi-906. Cells were treated for 15-30 hours with Trabectedin or Linsitinib or with combination. RNA was used to perform Low density expression arrays specifically targeting DNA damage and DNA repair genes 
Ewing Sarcoma cells to Trabectedin: An in vitro and in vivo assay A new promising therapy in ES

Ordoñez J and Amaral AT et al, Submitted, 2014 


\section{Abstract}

Ewing Sarcoma is a malignant bone and soft tissue tumor. Although patients with localized disease present favorable prognosis when submitted to multimodal therapies, patients with refractory/metastatic disease still have low overall survival rates. The development of novel treatment strategies is imperative, especially in these cases. Recent preclinical evidence suggested that ES bearing EWSR1-ETS fusions could be particularly affected by PARP inhibitors.

Here, we studied the genetic status, PARP1 mRNA levels and protein expression in a set of ES cell lines. Later on, we studied the anti-proliferativeeffects of PARPi Olaparib, Veliparib and Iniparib, being Olaparib the most effective agent. PARP1 is a transcriptional regulator of EWSR1-FLI1, and PARPi disrupt DNA damage repair (DDR) machinery. Trabectedin is a powerful agent that modulates EWSR1-FLI1 transcriptional functions provoking DNA damage. Therefore, we decided to combine Olaparib and Trabectedin. This combination resulted highly synergistic in terms of inhibition of cell proliferation, inducing also apoptosis, S phase-G2/M accumulation, and reduction of invasion. Drug combination also provoked $\mathrm{\gamma H} 2 \mathrm{AX}$ intranuclear accumulation as a result of DNA damage induction, DNA fragmentation and global DDR machinery deregulation. The effect of drug combination was corroborated in a xenograft model of ES developed in mice.

We concluded that the combination of these agents leads to a drastic deregulation of the global pathway of DDR machinery and could represent a promising therapeutic tool that should be explored more exhaustively in the clinic. 


\subsection{ES cells are especially sensitive to PARPi Olaparib, which induces G2/M} accumulation, independently of the $1 q$ status

Initially, we checked the status of PARP1, a gene located in the long arm of chromosome $1(1 \mathrm{q})$ in a panel of ES cell lines. To achieve this we labelled a home-made FISH probe specific for PARP1. We verified that ES cell lines previously described as 1q Gained, in fact, showed amplification of PARP1, whereas ES cell lines described as 1q Normal presented only two copies of PARP1 (Figure 45).
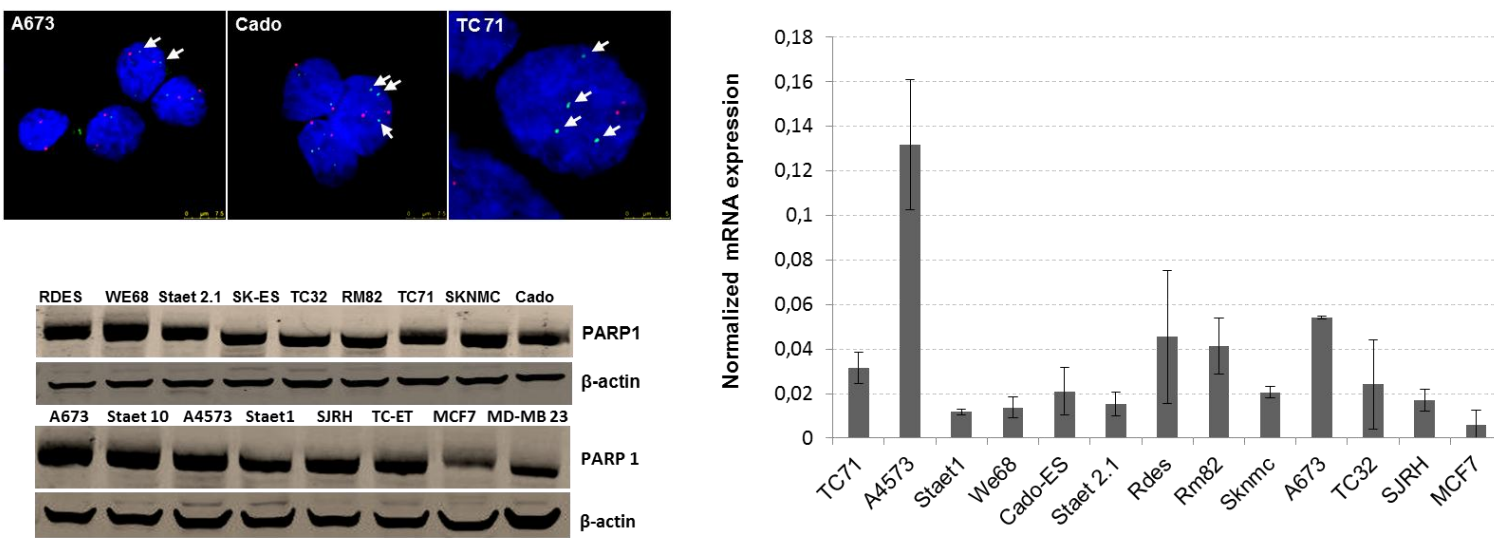

Figure 45. PARP1 expression in ES cell lines. FISH was performed by using homemade probes designed specifically for PARP1. To achieve this we selected BAC Clones RPII-964L17 and RPII-831N20. Green and red dots represent PARP1 gene copies and 1q centromeres, respectively. Arrows also indicate the green dots (PARP1 gene copies). ES cell lines previously described as 1q Gained showed extra-copies of PARP1 (Cado-ES and TC71 cell line) whereas A673, described as 1q Normal, showed only two copies of PARP1. q-RT-PCR performed with highly specific primers designed for PARP1 showed that PARP1 expression is heterogeneous along ES cell lines studied A4573 (1qG) and A673 (1q normal) are the cell line with a higher expression of PARP1. The breast cancer cell line MCF7 was used as a positive control. Western Blotting studies showed that ES cell lines express PARP1, under normal conditions. Here, breast cancer cell lines (MCF7 and MD-MB 23) were used as a positive control. Trabectedin resistant cell line, TC-ET cell line also expresses PARP1. 
Subsequently, cDNA analysis showed that all ES cells have different mRNA levels of PARP1 (Figure 45). Also, all cell lines studied expressed protein PARP1, using a specific antibody for isoform PARP1 (Figure 45).

Later, we studied ES cell lines sensitivity to a group of PARPi (Olaparib, Veliparib and Iniparib). Regarding proliferation inhibition, Olaparib was indeed more active than the other two drugs assayed showing lower $\mathrm{IC}_{50}$ levels of proliferation at 72 hours of exposure (high nM-low $\mu \mathrm{M}$ range, with a median of $1.995 \pm 0.46 \mu \mathrm{M})$. Veliparib was the second most effective agent with $\mathrm{IC}_{50}$ levels of proliferation on the $\mu \mathrm{M}$ range, presenting a median of $14.14 \pm 2.75 \mu \mathrm{M}$ (approximately 7 fold more than Olaparib). Finally, Iniparib was the less effective agent showing $\mathrm{IC}_{50}$ levels of proliferation also on the $\mu \mathrm{M}$ range but with a median of $74.95 \pm 5.02 \mu \mathrm{M}$ (approximately 38 fold more than Olaparib) (Figure 46).

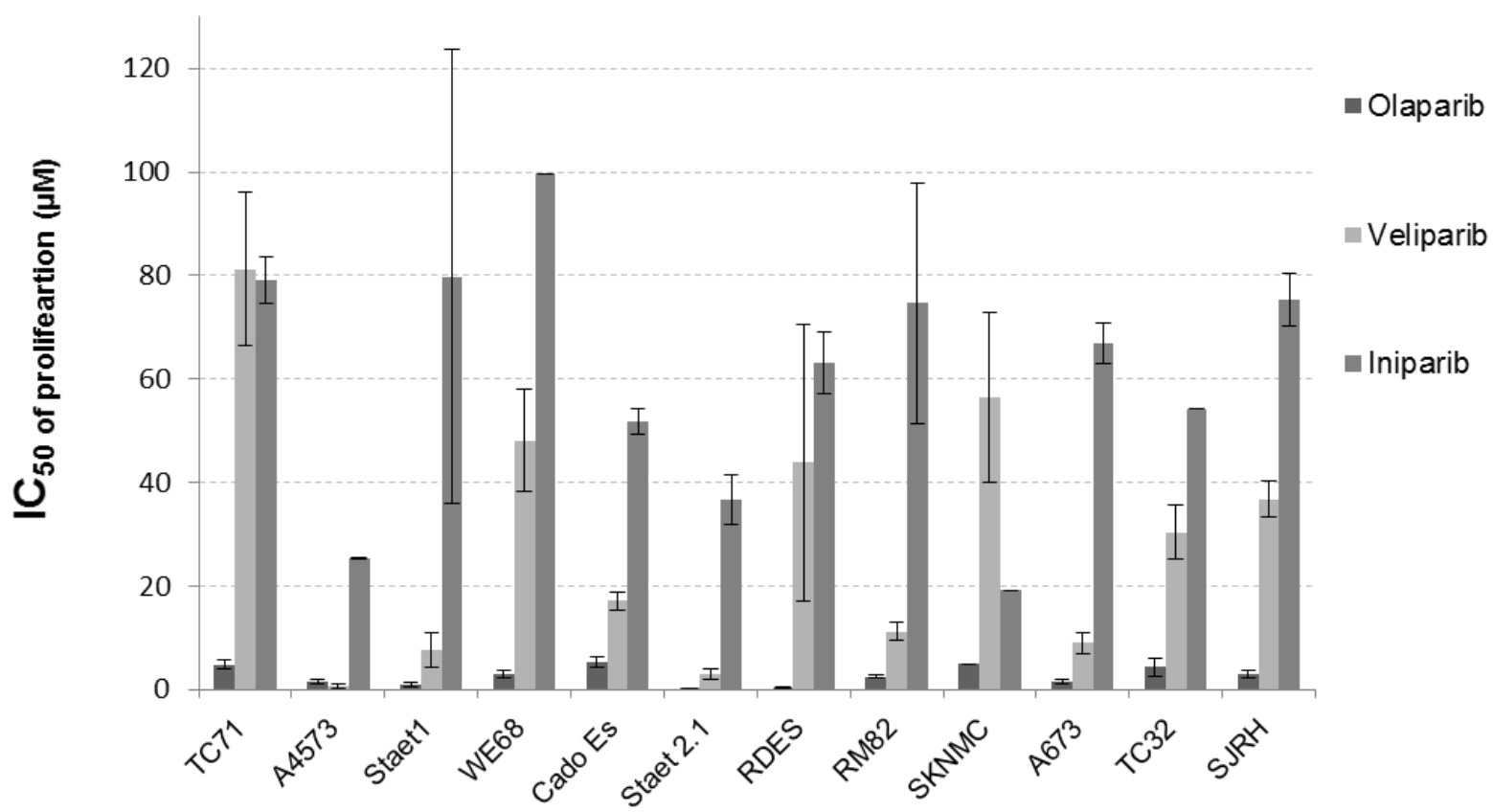

Figure 46. ES cell line Sensitivity to PARPi: Olaparib, Veliparib and Iniparib. ES cell lines were more sensitive to Olaparib than Veliparib or Iniparib, showing lower $\mathrm{IC}_{50}$ proliferation levels 
Given that PARP1, is placed in 1q chromosome, and that evidence published from our group described that some ES tumors and cell lines showed 1q gained, we search a possible correlation between the status of 1q (Gained or Normal) and the sensitivity to Olaparib (Figure 47). We observed a tendency for a higher sensitivity of 1qG cell lines; however this was not statistically significant, probably due to the low number of cell lines (Mann Whitney $U$ test, $p>0,05$ ).
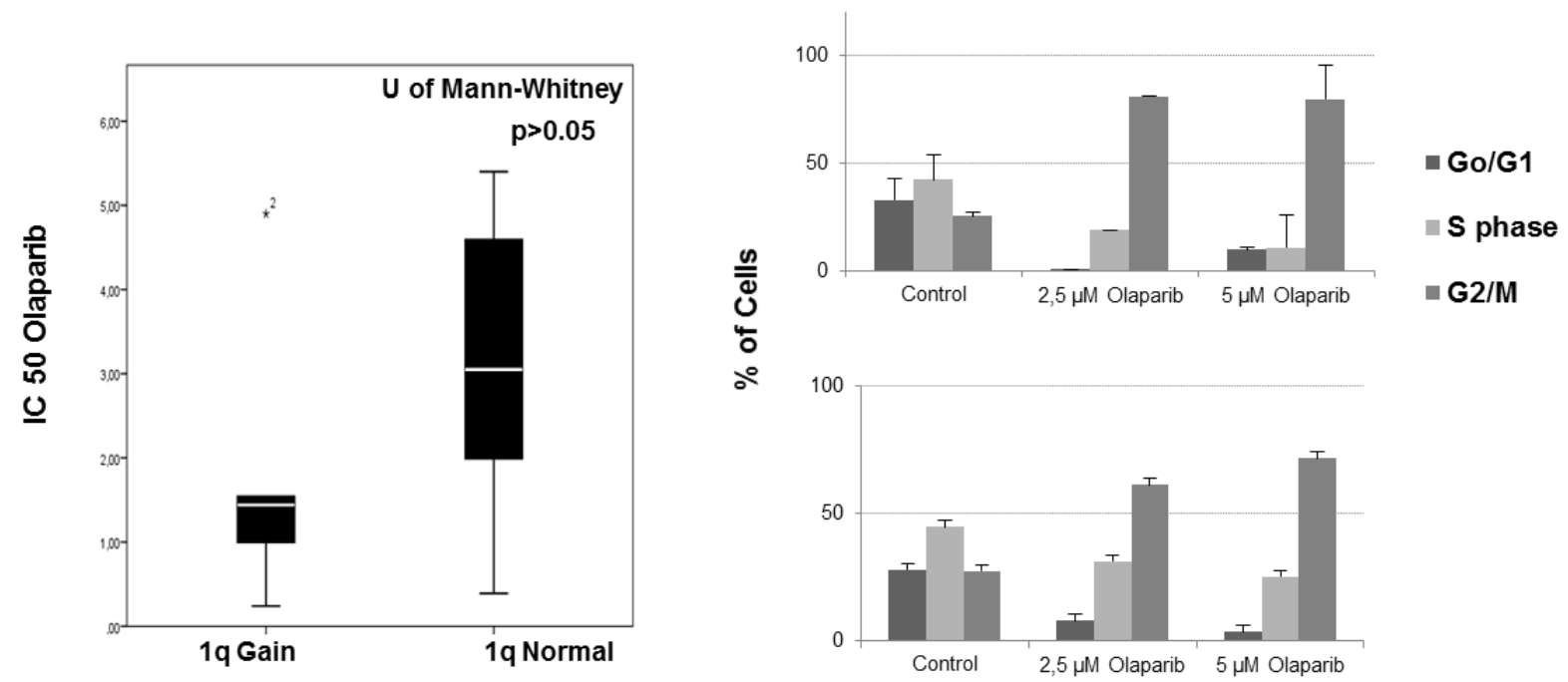

Figure 47. ES cell sensitivity induces $\mathrm{G} 2 / \mathrm{M}$ cell cycle accumulation and is not correlated to 1qG. (A) Sensitivity to Olaparib was not correlated with the status of 1q (gained/normal), $p>0,5$ (Mann-Whitney $U$ test). (B) Cell cycle analysis on A673 (1qN) and A4573 (1qG) after treatment with two different concentrations of Olaparib. In both cases Olaparib induced G2/M cell cycle arrest.

The correlation between the status of p53 (wild type or mutated) and sensitivity to Olaparib was not statistically significant either (Mann Whitney $U$, p>0,05) (Appendix, Figure 62S.A). In contrast with previous work, we observed that treatment with Olaparib for 6 days reduced the $\mathrm{IC}_{50}$ of proliferation (Appendix, Figure 62S.B)Furthermore, we studied the effects of Olaparib on the cell cycle profile using two ES cell lines, A673 (1qN) and A4573 (1qG) (Figure 
47). Both cell lines showed G2/M accumulation after treatment even at low doses of Olaparib (Figure 47).

\subsection{Combination of Olaparib and Trabectedin results is highly synergistic in} ES cell lines

Once observed that Olaparib was distinctively more cytotoxic than the other PARPinh, we studied the effects of the combination of Olaparib with Trabectedin. Here, ES cell lines $(n=13)$ were exposed to different combinations of both agents, with a constant ratio of 1:20.000 (Trabectedin: Olaparib) for 72 hours.

Cls were determined according to the Chou-Talalay method and, interestingly, synergistic effects were observed in all cell lines. Herein, only two cell lines (A4573 and WE68) showed nearly additive $\mathrm{Cl}$, whereas all the others presented clear synergistic levels. (Table XXIV).

Later on, we studied the effects of this drug combination on apoptosis induction via caspases -3 and -7 activation after 48 hours of drug exposure, as well as the cell cycle effects after 24 hours of drug exposure on two cell lines, RM82 and TC71. We observed that the drug combination (250 pM of Trabectedin and $5 \mu \mathrm{M}$ of Olaparib) increased the apoptotic rate in the TC71 cell line. Furthermore a phase-G2/M accumulation and elimination of the G0/G1 phase was also observed after treatment with the drug combination in the RM82 cell line (Figure 48). In this cell line, treatment with drug combination only provoked mild apoptosis. 
Table XXIV. Combination of Olaparib and Trabectedin in ES cell lines

\begin{tabular}{ccc}
\hline Cell lines & Cl & Effects \\
\hline A673 & 0.77 & Synergism \\
& & Nearly \\
A4573 & 0.93 & additive \\
CADO & 0.4 & Synergism \\
RDES & 0.73 & Synergism \\
RM82 & 0.69 & Synergism \\
SJRH & 0.11 & Synergism \\
STAE 1 & 0.64 & Synergism \\
STAE 2.1 & 0.49 & Synergism \\
TC71 & 0.4 & Synergism \\
TTC466 & 0.25 & Synergism \\
SK-ES-1 & 0.81 & Synergism \\
SK-N-MC & 0.008 & Synergism \\
Cell lines with the exception of A4573 \\
additive (values close to 1 )
\end{tabular}


TC71

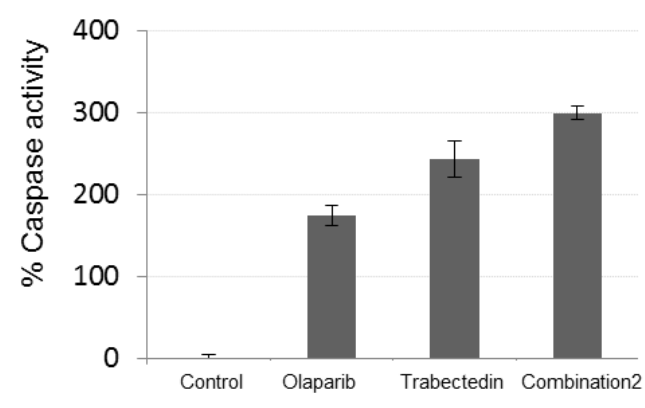

RM82

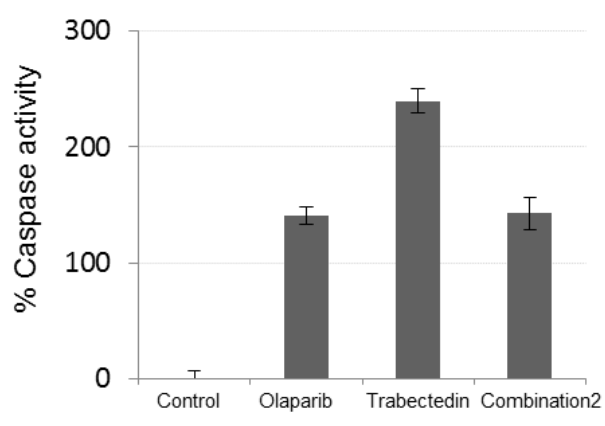

TC71

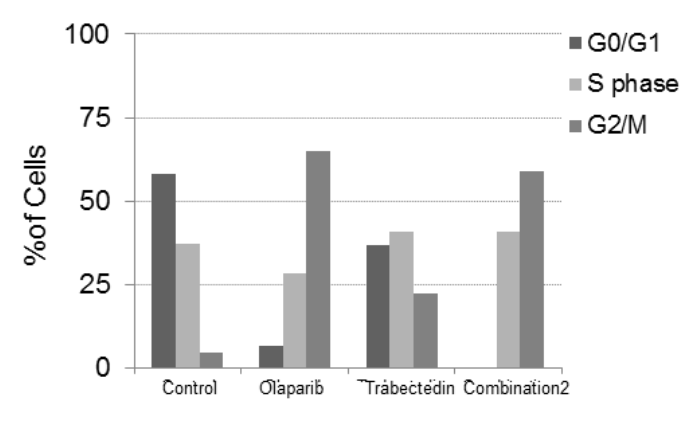

RM82

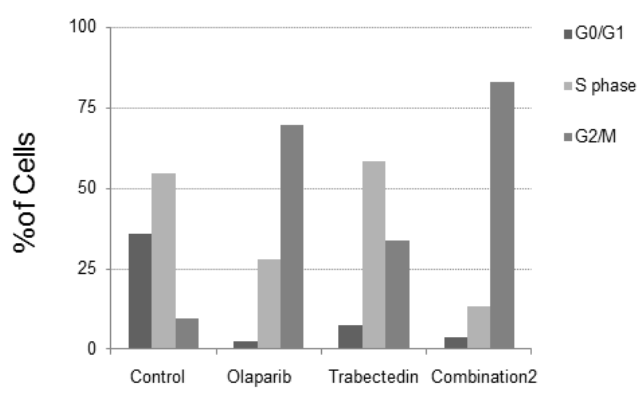

Figure 48. The combination of Trabectedin and Olaparib: effects on caspases activity and cell cycle profiling. (A) Graphics represents data obtained in the apoptosis assay by studying caspases -3 and -7 activity. Treatment with Olaparib increases apoptosis as compared to control (141\% in RM82 and $174 \%$ in TC71 cell line). Treatment with Trabectedin induced a higher level of apoptosis (240\% and $242 \%$ in RM82 and TC71 cell line respectively). Interestingly, the combination of Olaparib and Trabectedin in TC71 cell line, induced clear increase in apoptosis as compared with the effects induced by drugs alone (299\%). (B) Graphics representing data from cell cycle analysis after 24 hours of treatment, in the RM82 and TC71 cell line. Trabectedin alone induced S-phase accumulation, whereas Olaparib induced strong G2/M accumulation .The combination induced accumulation on the S-phase and G2/M and significant decrease or even elimination of G0/G1 phase

The effects over cell migration after 72 hours of drug exposure and cell invasion after 48 hours of exposure in the RM82 cell line were also analyzed. (Appendix, Figure 63S) No effects on cell migration were observed. 


\subsection{The combination of Olaparib and Trabectedin enhances DNA damage}

\section{induction}

DNA damage induction was evaluated by the presence of intranuclear foci of $\mathrm{yH} 2 \mathrm{AX}$ and 53BP1 after short (8 hours) and long (24 hours) drug exposure. Initially, cells were treated with Trabectedin and/or Olaparib (combination 2: 250 pM of Trabectedin and $5 \mu \mathrm{M}$ of Olaparib) for 8 hours. Trabectedin induced a stronger DSB accumulation when compared to Olaparib alone. Nevertheless, the combination of both agents resulted in a clear enhancement on DSB formation (Figure 49).

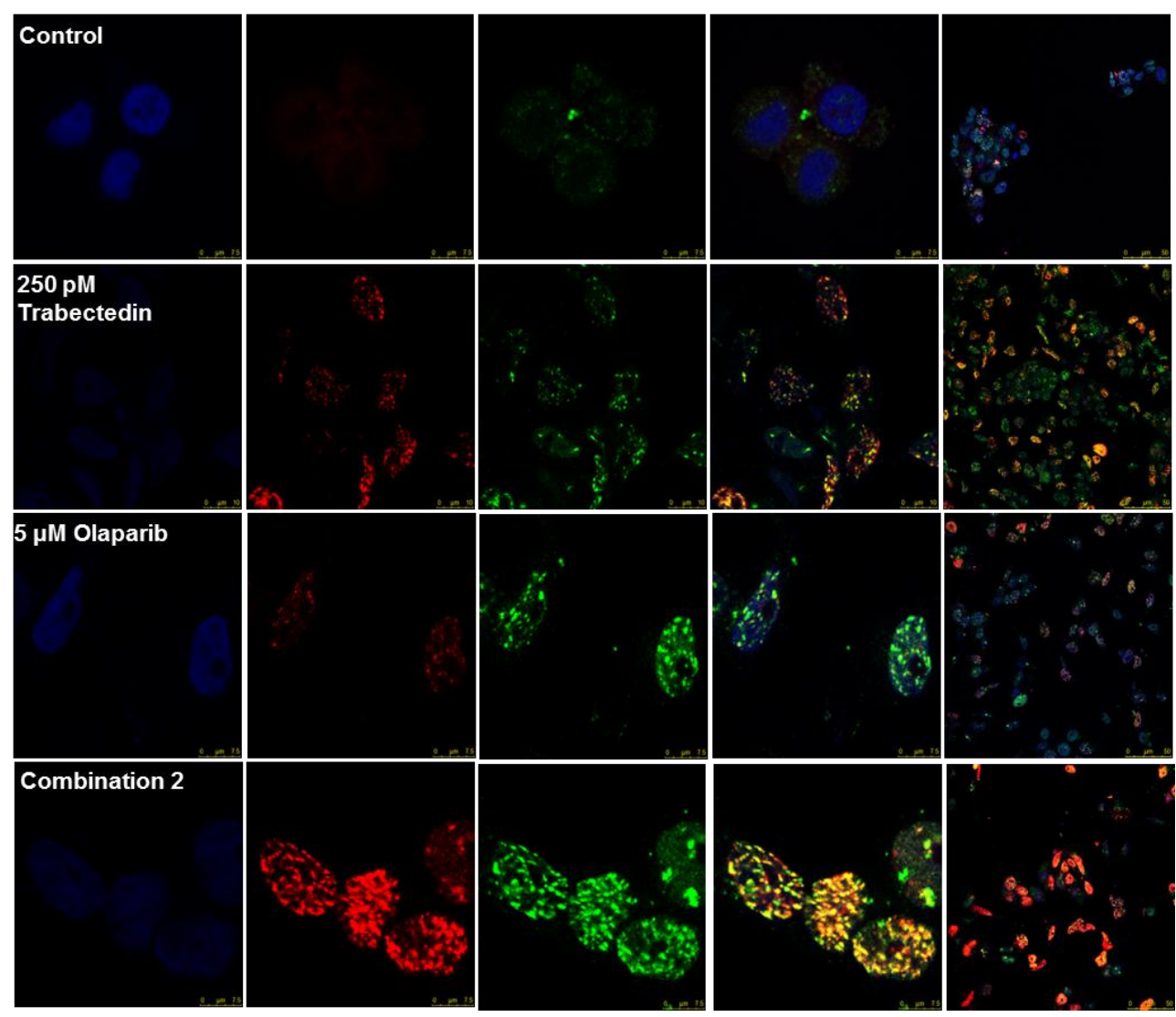

Figure 49. DNA damage induction studies: Short-term damage induction. Pictures represent data obtained in the study of induction of DSB evaluated by the presence of intranuclear $\mathrm{\gamma H} 2 \mathrm{AX}$ and 53BP1 foci. Short-term DNA damage induction. TC71 cells were treated for 8 hours with Trabectedin (250pM) and/or Olaparib $(5 \mu \mathrm{M})$. Trabectedin alone induced more DSB than Olaparib alone. The combination of Trabectedin and Olaparib showed an increased effect on DSB induction, with increased presence of $\mathrm{\gamma H} 2 \mathrm{AX}$ and 53BP1 foci in the nucleus 


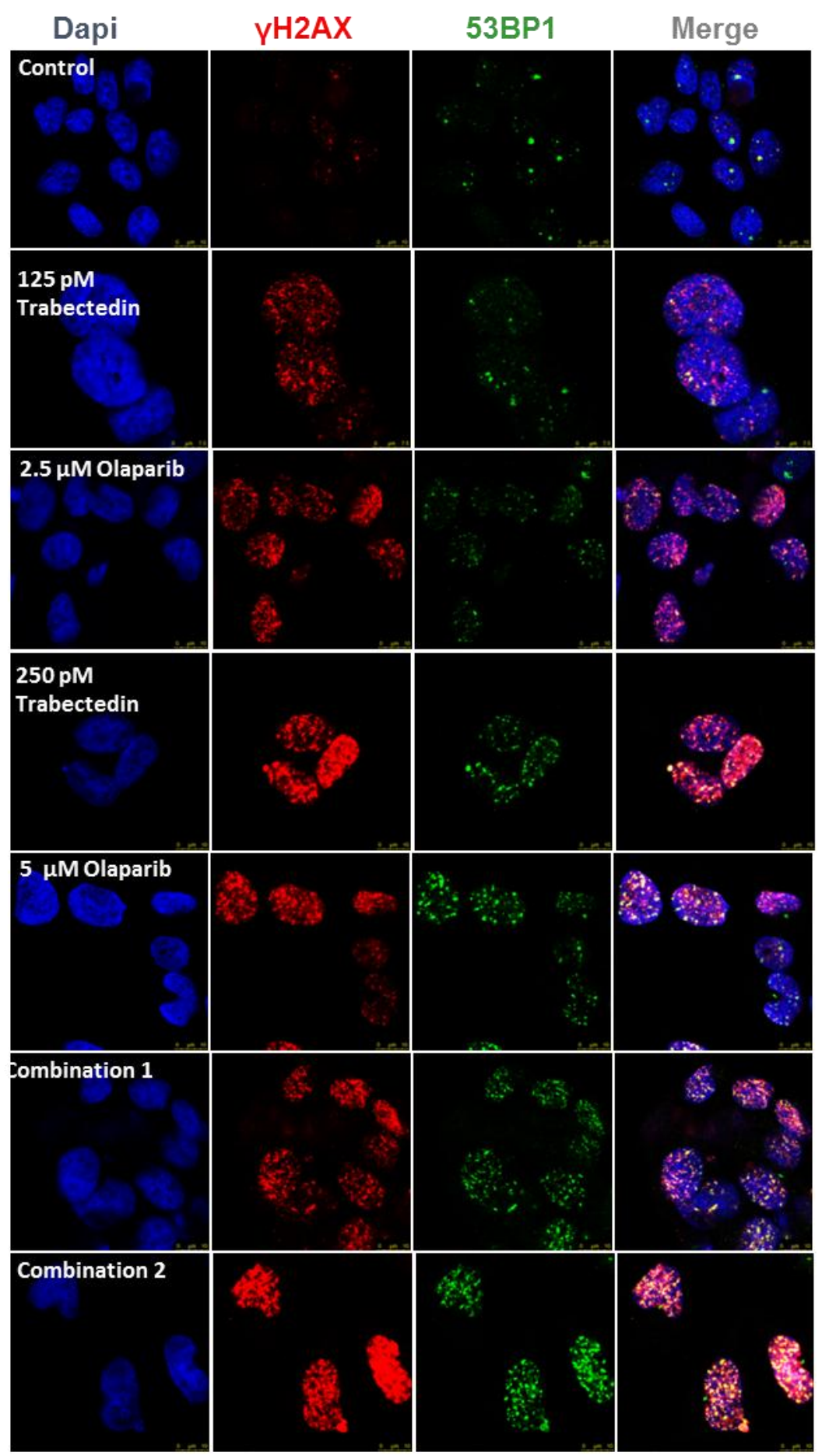

Figure 50. DNA damage induction studies: Long-term damage induction. Longterm DNA damage induction, evaluated on TC71 cells treated for 24 hours with Trabectedin (125pM, 250pM) and/or Olaparib $(2,5 \mu \mathrm{M}, 5 \mu \mathrm{M})$, which suggests that Olaparib probably needed more time to generate DSB. Again, the combination showed an increase on DSB as compared to the drugs alone 
We also evaluated the presence of $\mathrm{yH} 2 \mathrm{AX}$ and 53BP1 after 24 hours of drug exposure, and here we also included intermediate concentrations of $125 \mathrm{pM}$ of Trabectedin and $2.5 \mu \mathrm{M}$ of Olaparib both alone and in combination. Again, we observed that the drug combinations resulted in cumulative effects of DNA damage through induction of DSB. (Figure 50)

Moreover, DNA fragmentation assays performed at 24 hours and 48 hours of drug exposure, demonstrated that both drugs are able to induce enough damage to induce DNA fragmentation, especially after 24 hours of treatment.

Here, DSB induced DNA fragmentation is observed by the presence of a smear just below the intact DNA (represented by *) whereas the smaller fragments (represented by ${ }^{* *}$ ) are produced exclusively by apoptosis induction (Figure 51).

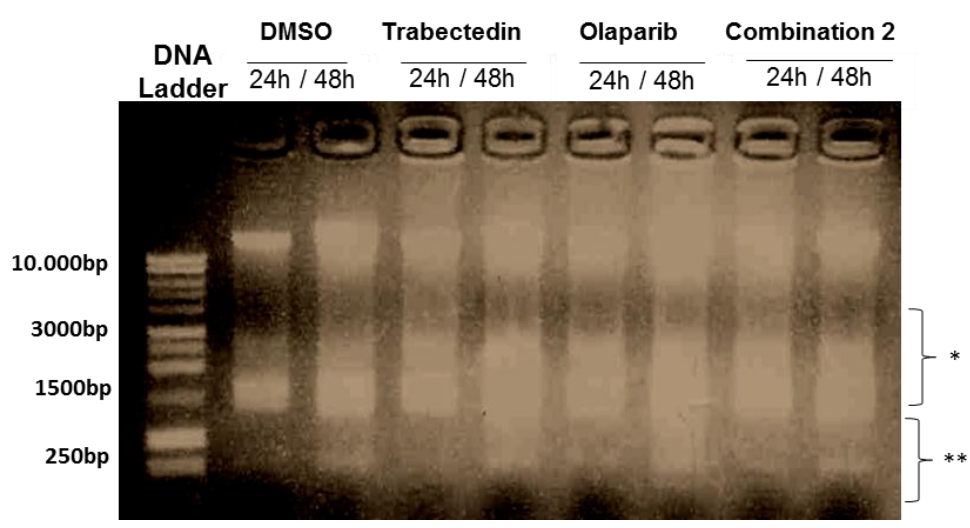

Figure 51. DNA damage induction studies: Fragmentation Studies. These assays were performed after 24 hours - 48 hours drug exposure. Although DNA fragmentation was also observed in the control (DMSO), Trabectedin and/or Olaparib induced higher DNA fragmentation, especially after 24 hours of drug exposure where more fragmentation can be observed in comparison to the control. In all cases combination 1 means (Trabectedin 125pM and Olaparib 2,5 $\mu \mathrm{M})$; combination 2 means (Trabectedin $250 \mathrm{pM}$ and Olaparib $5 \mu \mathrm{M}$ ) 


\subsection{Olaparib and/or Trabectedin provoked the deregulation of DDR machinery}

After demonstrating that the combination of Olaparib and Trabectedin leads to DNA damage by DSB accumulation, we next proceeded to study the effects on the regulation of the DDR machinery in TC71 cells. To achieve this we used the low density arrays (DDR PCR Array; PAHS-042Z, Qiagen). (Appendix) After 16.5 hours of treatment with Olaparib and/or Trabectedin, RNA was extracted and changes in the mRNA levels regarding DNA damage induction and DDR mechanisms were analyzed and compared to the control conditions. Treatment with Trabectedin revealed overexpression of $\mathrm{HR}$ related molecules such as BRCA1, RAD18, RAD52, BRCA2 and BRIP1 (BRCA1/2 associated molecules) as well as POLD3 (DNA polymerase delta subunit 3); NEIL3 (endonuclease VII-like 3) involved in DNA binding and double strand binding; RFC1 (replication factor C) involved in GC-NER DSB reparation; OGG1; XRCC1 involved in DNA and protein binding and strand break correction and UNG, a DNA glycosilase involved in the BER pathway (Table XXV.A)

Treatment with Olaparib, on the other hand, also induced the expression of HR related proteins EXO1, RAD54L, RAD51B and RAD18, together with BRIP1, RFC1, PARP3, XRCC, POLD3 and PARP3. The global deregulation of DNA damage and repair machinery was clearly observed in the combination situation where HR proteins RAD54L, EXO1, PARP2, BRCA1, RAD18, BRCA2, RAD51, RAD 50, RPA1 were overexpressed (Table XXV.A). Changes in expression in this array were mostly correlated with DSB repair by the NER pathway ( $p$ value: $1.02 E-23$ ), the NHEJ pathway ( $p$-value: $1.81 \mathrm{E}-23$ ) the HR 
pathway ( $p$-value: 4.41E-18) and also BRCA1 and BRCA1 related pathways ( $p$ values $4.52 \mathrm{E}-18$ and $2.24 \mathrm{E}-17$

Table XXV. Expression changes in DNA damage repair genes in ES cells from the TC71 cell line.

A.

\begin{tabular}{|c|c|c|c|c|c|}
\hline \multicolumn{6}{|c|}{ Up-Regulated } \\
\hline Trabectedin & Exp value & Olaparib & Exp value & Combination 2 & Exp value \\
\hline BRCA1 & 1.644 & EXO1 & 1.927 & RAD54L & 2.548 \\
\hline POLD3 & 1.394 & BRIP1 & 1.904 & TOP3A & 2.501 \\
\hline BRIP1 & 1.382 & TOP3A & 1.903 & EXO1 & 2.466 \\
\hline RAD18 & 1.347 & RAD54L & 1.886 & BRIP1 & 2.338 \\
\hline NEIL3 & 1.346 & RFC1 & 1.700 & POLD3 & 2.160 \\
\hline RFC1 & 1.247 & PARP3 & 1.625 & RFC1 & 2.159 \\
\hline OGG1 & 1.229 & RAD51B & 1.613 & PARP2 & 1.899 \\
\hline RAD52 & 1.205 & XRCC1 & 1.609 & BRCA1 & 1.869 \\
\hline XRCC1 & 1.166 & POLD3 & 1.597 & RAD18 & 1.819 \\
\hline UNG & 1.160 & RAD18 & 1.550 & XRCC1 & 1.810 \\
\hline \multicolumn{6}{|c|}{ Down-Regulated } \\
\hline Trabectedin & Exp value & Olaparib & Exp value & Combination 2 & Exp value \\
\hline PMS2 & -625.000 & RAD23B & -1.302 & MSH4 & -3.358 \\
\hline RAD51B & -44.444 & NEIL2 & -1.089 & XRCC4 & -2.477 \\
\hline RAD51 & -10.846 & NEIL1 & -1.046 & RAD51B & -1.892 \\
\hline MSH5 & -8.795 & DMC1 & -1.044 & MGMT & -1.873 \\
\hline TOP3B & -8.532 & ATXN3 & -1.044 & RAD23B & -1.423 \\
\hline PMS1 & -8.019 & NTHL1 & -1.037 & ATXN3 & -1.294 \\
\hline LIG1 & -5.952 & XPC & -1.027 & SMUG1 & -1.193 \\
\hline XPA & -4.653 & PARP1 & -1.017 & NEIL2 & -1.159 \\
\hline FEN1 & -4.137 & XPA & -1.005 & APEX1 & -1.153 \\
\hline XRCC6 & -4.050 & PMS1 & -1.003 & NTHL1 & -1.143 \\
\hline
\end{tabular}

Table depicts the most significantly up/down expressed genes in each treatment condition. Numeric data refers to levels of expression after comparing with control) 
B

\begin{tabular}{ccc}
\hline Pathways & Ratio & p-value \\
\hline NER & $14 / 35(0.4)$ & $1.02 \mathrm{E}-23$ \\
NHEJ & $11 / 16(0.688)$ & $1.81 \mathrm{E}-23$ \\
HR & $9 / 16(0.562)$ & $4.41 \mathrm{E}-18$ \\
BRCA1 & $13 / 63(0.206)$ & $4.52 \mathrm{E}-18$ \\
HBCS & $15 / 119(0,126)$ & $2.24 \mathrm{E}-17$
\end{tabular}

Table representing the top pathways covered by the array. Major pathways affected are involved in the repair of DSB. Ratio indicates the number of affected genes of a pathway/total number of genes of a pathway. p-value is an estimation of the likelihood that the correlation between the number of affected genes in our study which participate in a pathway and the total number of genes which are known to be associated with that pathway is due to random association. P-values less than .05 indicate a statistically.

After a deep analysis of internal negative and positive controls, pathway analysis using the software IPA, discriminated the most significant upregulated molecules (in red) and downregulated (in green) with respect the major canonical pathway affected, DNA damage and cell cycle regulation (Table XXV.B). The combination strongly induced a major deregulation of the molecules involved in these pathways. 
Trabectedin

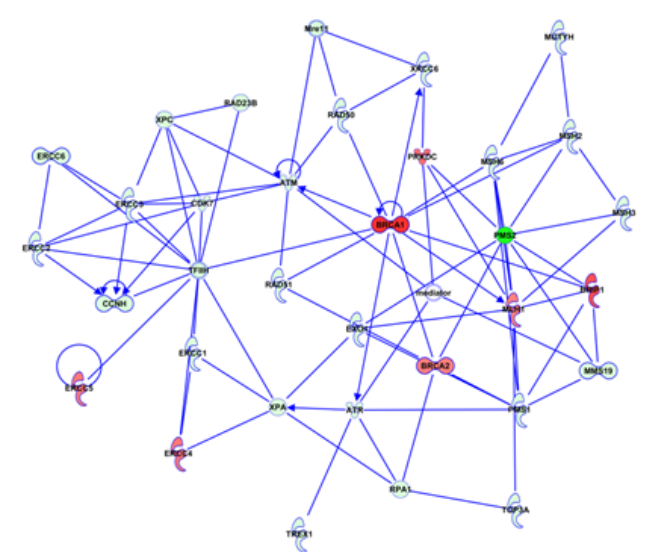

\section{Olaparib}

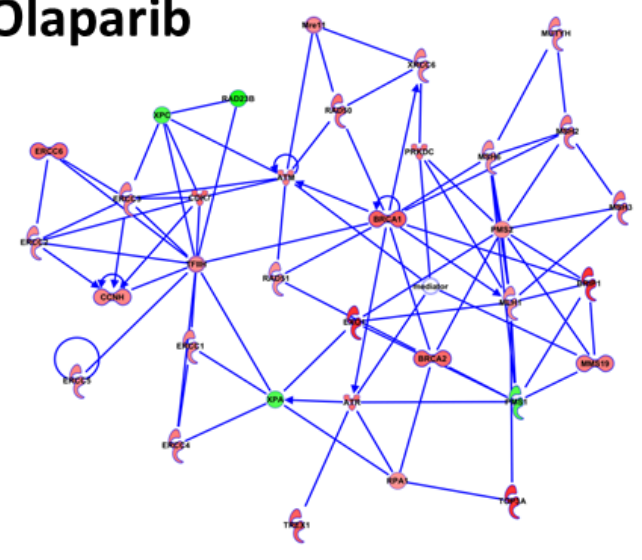

\section{Combination 2}

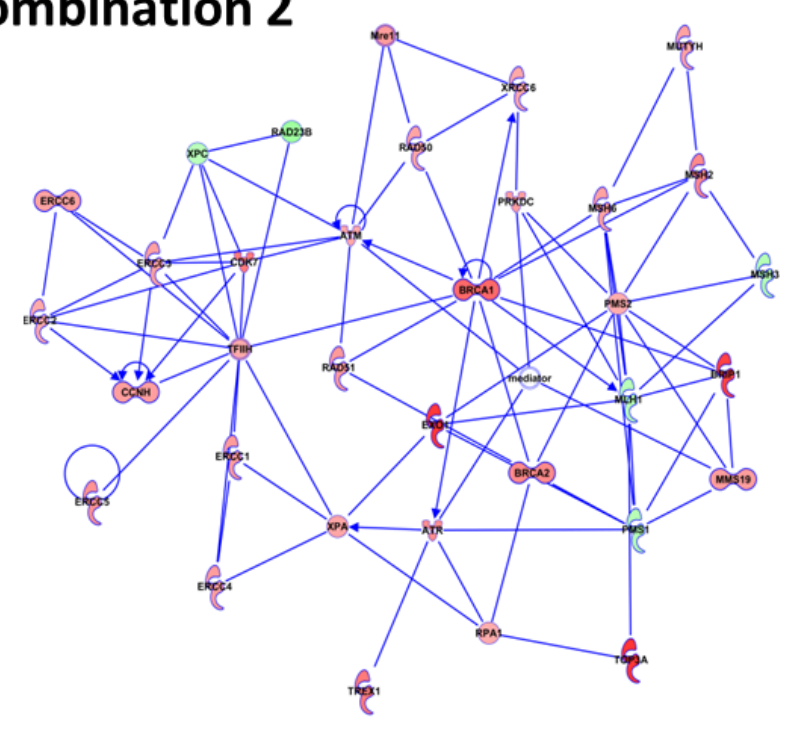

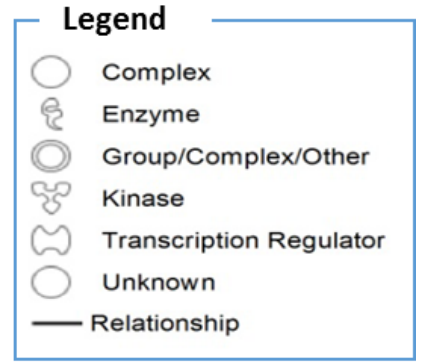

Figure 52. Schematic representation of gene interactions in cells treated with Trabectedin, Olaparib or combination of Olaparib and Trabectedin. Images obtained after performing the Ingenuity pathway analysis. Over-expressed genes are highlighted in red, and the down regulated genes are highlighted in green. Central genes involved in HR machinery are particularly highlighted such as BRAC1, EXO1, RAD51, and BRCA1 among others. Combination of Olaparib and Trabectedin drastically deregulated the global DNA damage and repair pathway. 


\subsection{Drug combination dramatically reduced tumor growth in an in vivo model}

Given the results obtained in the in vitro study, we decided to test the antitumoral effects of combination of Trabectedin and Olaparib in a xenograft model of ES developed in NOD/SCID mice. Xenografts were generated by subcutaneous injection of TC71 cells into mice. Animals were randomized in 4 groups and treated for three weeks with: i) Vehicles (control group); ii) Trabectedin alone $(0.15 \mathrm{mg} / \mathrm{Kg}$ IV once a week); iii) Olaparib alone $(100 \mathrm{mg} / \mathrm{Kg}$ BID IP and iv) Olaparib (100mg/Kg BID IP) plus Trabectedin $(0.15 \mathrm{mg} / \mathrm{Kg}$ IV once a week). After the first week the Olaparib group was divided in two groups $(n=5)$, iii) where $50 \%$ of the animals $(n=5)$ were treated with Olaparib alone $(100 \mathrm{mg} / \mathrm{Kg}$ BID IP), and the other $\mathrm{v})(\mathrm{n}=5)$ were treated with the Olaparib plus Trabectedin drug combination. After three weeks of treatment, mice were sacrificed and tumors were extracted, weighted and evaluated by histopathology and IHC.

The evolution of tumor volume after randomization and the tumor weight at the end of the study (18 days after treatments started to be administered), depicted on Figure 53 respectively, were evaluated in all the groups.

Although Trabectedin and Olaparib alone were able to reduce tumor volume as compared with the control group, these differences were not statistically significant $(p>0.05)$. However, the combination of both these drugs dramatically reduced tumor growth with respect to the control group $(p<0.05)$. In fact, tumors were even smaller at the end of the study than they were at the moment of randomization, just before starting with the treatment. 
A

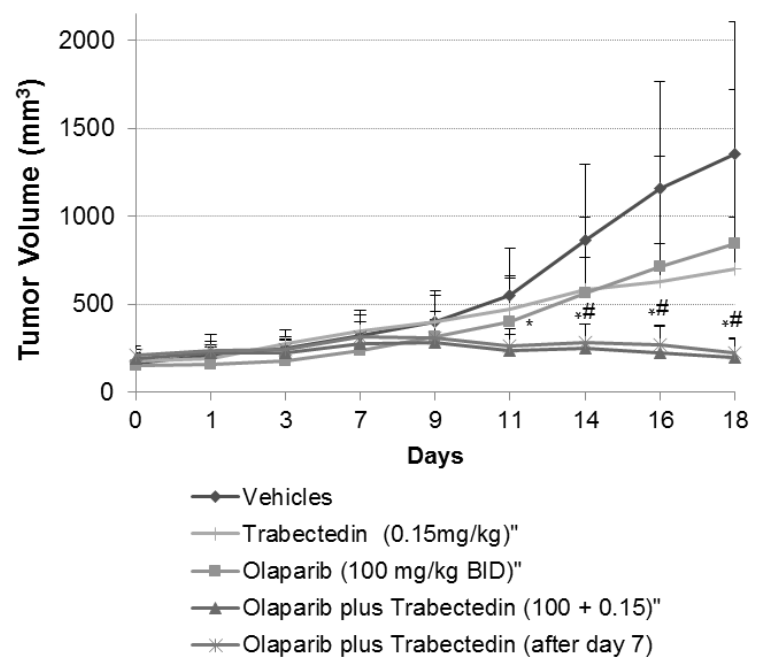

B

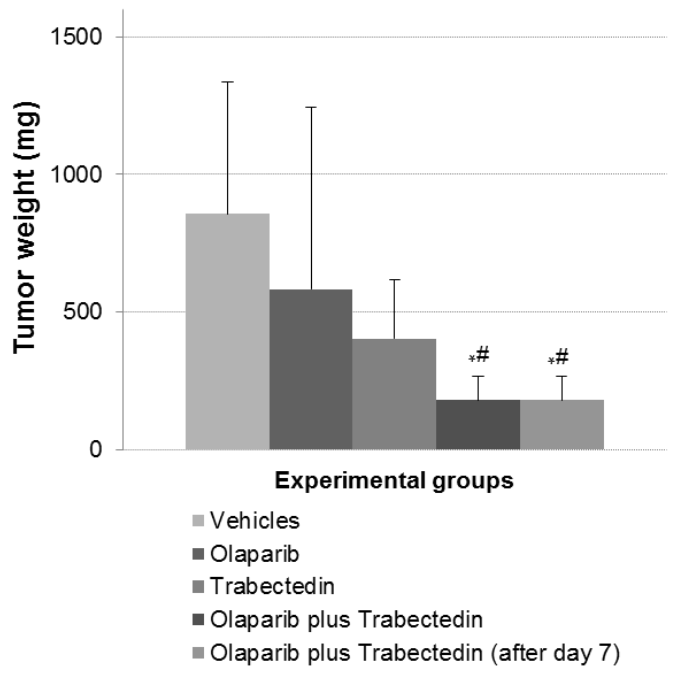

Figure 53. In vivo model using the TC71 cell line. (A) Graphic representing the evolution of the tumor growth in the different groups of treatments. Tumor growth was clearly halted by the combination of Olaparib and Trabectedin (group V) and when Trabectedin was started to be administered one week later (group IV) (B) Graphic representation of mean tumor weights at the end of the study (18 days after treatment started to be administered) in each of the groups of treatment. Tumor weight was clearly reduced, especially in groups IV and V (* or \#, $p<0.05$; *, Comb 1 or Comb 2 vs control; \#, Comb 1 or comb vs Olaparib alone or Trabectedin alone). Comb 1 means (Olaparib $100 \mathrm{mg} / \mathrm{Kg}$ and Trabectedin $0.15 \mathrm{mg} / \mathrm{kg}$ (group V). Comb2 means (Olaparib $100 \mathrm{mg} / \mathrm{kg}$ and Trabectedin $0.15 \mathrm{mg} / \mathrm{kg}$ started to be administered after day 7 (group IV)

The histopathological analysis from representative tumors of each group of mice, revealed significant morphological changes, starting from the control, Olaparib plus Trabectedin (group 4), and finishing in Trabectedin plus Olaparib group (group 5).

Drug induced morphological changes were characterized by the presence of ES cells with large vacuoles, clear and enlarged cytoplasm as well as conspicuous nucleoli (Figure 54). Necrosis was observed in animals of all groups; however, the mitotic index was higher in the control in comparison to the treated groups. In fact, the necrosis-viable tumor ratio was much higher in 
the treated groups. In the control groups, necrosis appears to be slowly reached, whereas in the treated groups it was much more extensive largely installed, suggesting a rapid process of cell death induction.

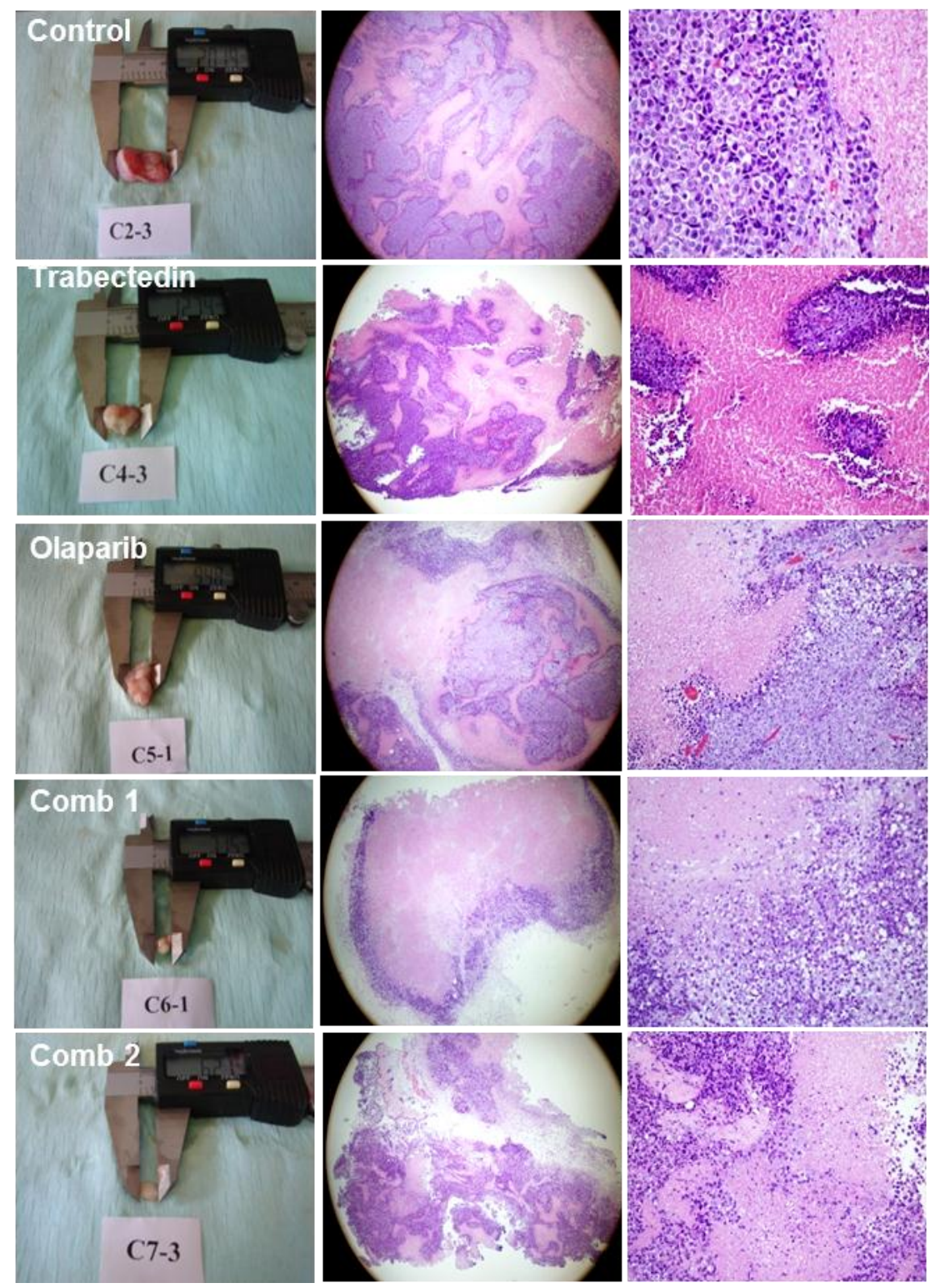

Figure 54. In vivo model using the TC71 cell line: Histopathological analysis. Representative pictures showing in columns from the left to the right: tumor in mice, tumors extracted and $4 \mathrm{X}$ and $20 \mathrm{X} \mathrm{H}$ \&E representative areas of the tumors of each group of treatment respectively.

Later on, we studied any possible morphological lesions associated to toxicity with these therapeutic regimens. Representative areas from kidneys and 
livers extracted from animals of the five groups were evaluated by H\&E in search of nephrotoxicity / hepatotoxicity effects (Figure 55).

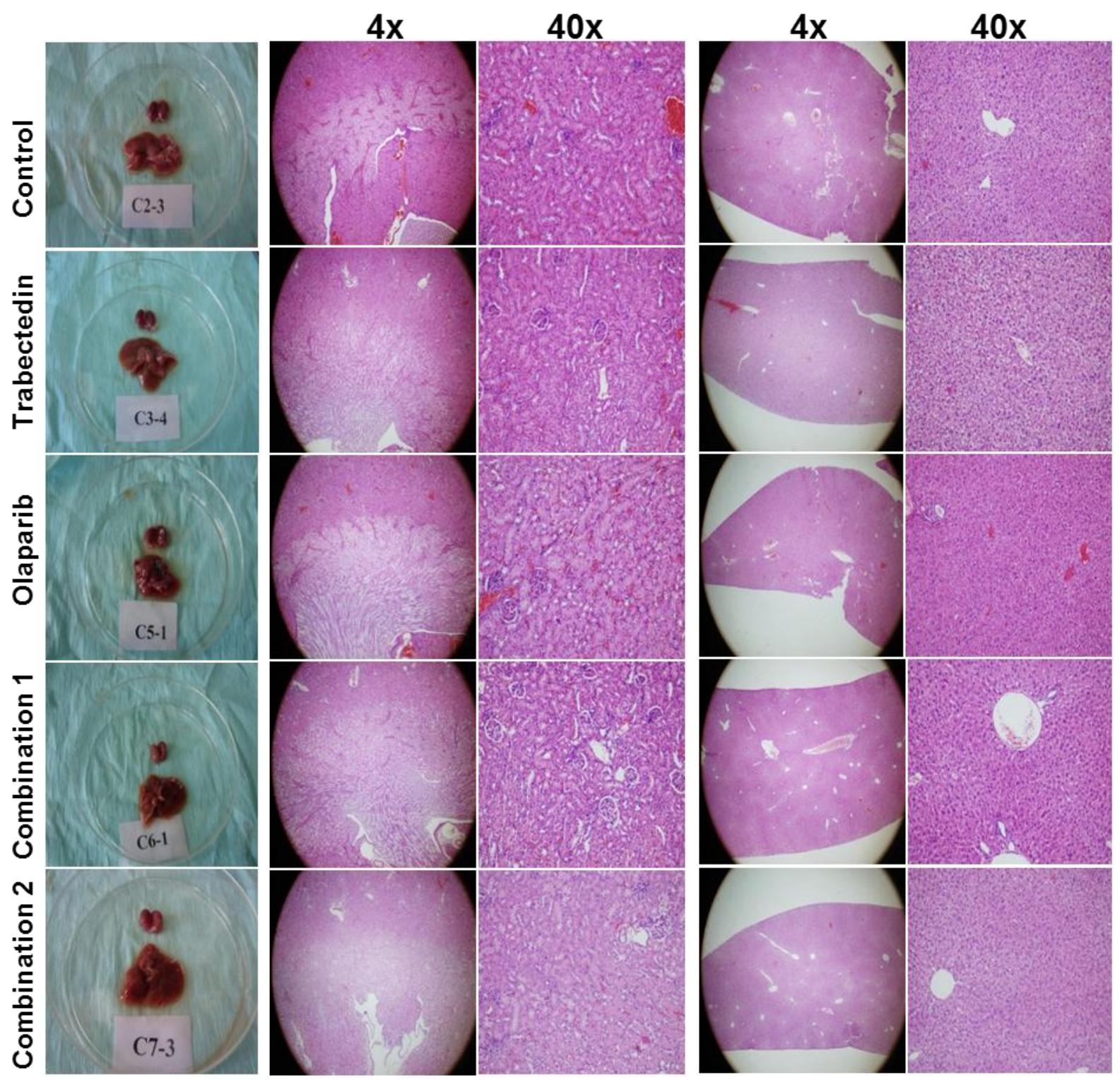

Figure 55. In vivo model using the TC71 cell line: Toxicity analysis. First panel represents kidneys and liver at the moment of extraction. Nephrotoxicity was not observed in any group (4X, 40x). No hepatotoxicity was observed $(4 \mathrm{x}, 40 \mathrm{x})$.

Interestingly, all livers and kidneys showed a normal morphology with no signs of toxicity (Figure 2S). The histopathological analysis of the kidneys revealed no evident differences between groups. Glomeruli and all other 
structures from the cortical area were very well preserved in all experimental groups. Accordingly, no morphologic changes were observed in any of the liver samples studied. No signs of toxicity were observed in the hepatocytes. The cells showed nuclei not activated and pink cytoplasm. Portal spaces and bile ducts were normal and similar in the control/treated samples.

\subsection{The drug combination increased DNA damage induction and DDR activation also in the in vivo model}

A representative number of tumor areas from tumors of all groups in the in vivo study was evaluated by $\mathrm{IF} / \mathrm{IHC}$ in order to assess the effects of treatments. The following parameters were studied: apoptosis (fragmentationlate apoptosis and PARP cleavage) (TUNEL assay and cleaved PARP); proliferation (Ki67), DNA damage induction ( $\mathrm{\gamma H} 2 \mathrm{AX}$ ) and DDR activation (BRCA2).

Necrosis was seen in tumors from all groups but it was more extensive in the combination groups. We also observed that proliferation index was higher in the control and Olaparib group when compared to Trabectedin alone group and the combination groups. (Figure 56 right column). These results are in accordance with data from TUNEL assays, where late apoptosis due to DNA fragmentation was mostly observed in representative tumoral areas from animals treated with Trabectedin alone or in tumors from animals treated with the combination of drugs (Figure 56 left column). The study of PARP cleavage revealed only a slight staining on tumors from the Olaparib group. (Figure 55, middle column) 


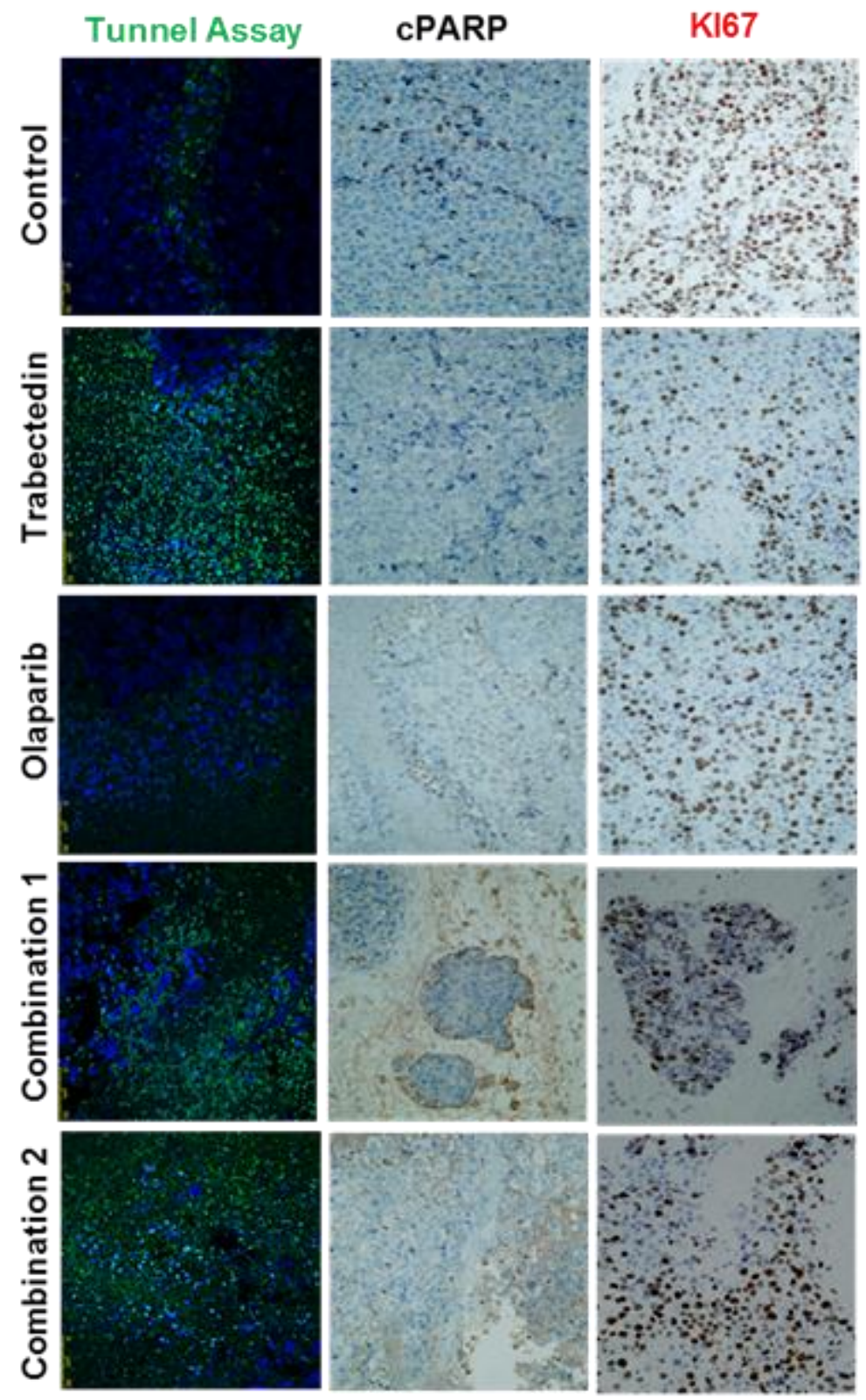

Figure 56. Immunofluorescence and Immunohistochemistry study of tumor xenografts: Apoptosis, proliferation. Immunohistochemical study of representative tumor areas in the different groups of treatment. The following parameters were evaluated: apoptosis (TUNEL assay and PARP cleavage); proliferation (KI67); we observed that proliferation index was higher in the control group and, Olaparib group when compared to Trabectedin alone and mainly to the combination groups. Furthermore, in the TUNEL assay, where late apoptosis due to DNA fragmentation was mostly observed in tumors from the Trabectedin group alone and from both Olaparib and Trabectedin combinations. The study of PARP cleavage only revealed a slight staining in tumors from the Olaparib group. 


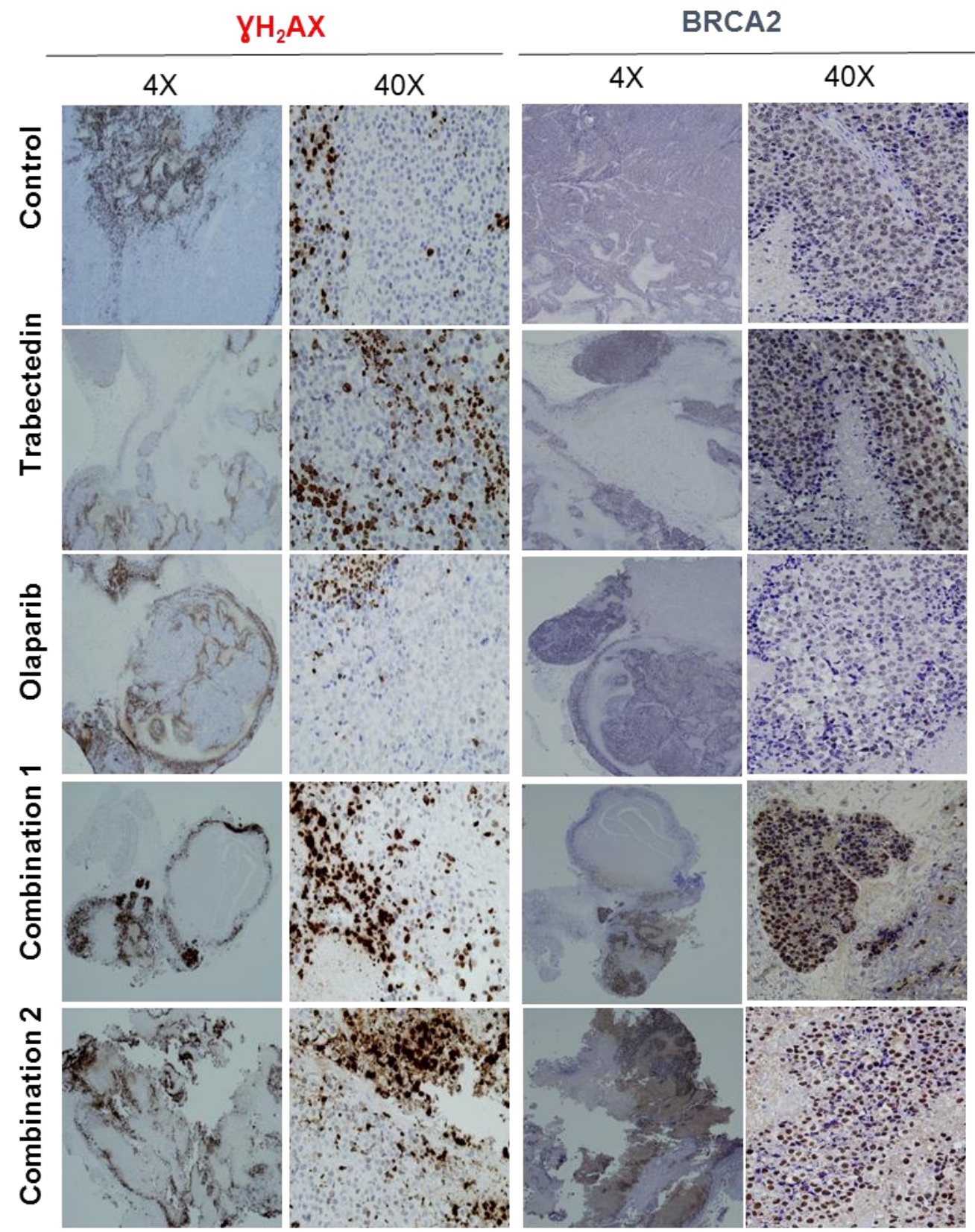

Figure 57. Immunofluorescence and Immunohistochemistry study of tumor xenografts: DNA damage Induction and repair. Regarding DNA damage induction, the presence of intranuclear $\mathrm{pH} 2 \mathrm{AX}$ staining was much higher in tumors from the Olaparib and Trabectedin combinations. In fact, all proliferating cells showed a high staining of $\mathrm{yH} 2 \mathrm{AX}$ in the combinatory regimens. This observation is in accordance with overexpression of BRCA2 which is the HR effector molecule. Treatment with Olaparib \& Trabectedin (groups IV and V) resulted in a much more intense and focal staining, especially in tumors from group V. There was some expression observed in the Trabectedin/Olaparib alone groups, however, it was more diffuse and less intense in tumors from Olaparib group and diffuse but more intense in tumors form Trabectedin group. Combination 1 means (Olaparib $100 \mathrm{mg} / \mathrm{Kg}$ \& Trabectedin $0.15 \mathrm{mg} / \mathrm{kg}$ ). Combination 2 means (Olaparib $100 \mathrm{mg} / \mathrm{kg} \&$ Trabectedin $0.15 \mathrm{mg} / \mathrm{kg}$ started to be administered after day 7 ). 
Interestingly, DNA damage induction assessed by the intranuclear pH2AX staining was much higher in animals of both combination groups. In fact, all proliferating cells showed a high staining of $\mathrm{pH} 2 \mathrm{AX}$ in the combinatory regimens (Figure 57).

This observation correlated with overexpression of HR effector molecule, BRCA2. Treatment with Olaparib \&Trabectedin (groups iv and v) resulted in a much more intense and focal staining of BRCA2, especially in group 5 . In the tumors from animals treated with only one drug (Trabectedin or Olaparib) there was also some expression of BRCA2, it was more diffuse and less intense in the case of Olaparib group, and diffuse and a bit more intense in the case of tumors from the Trabectedin group (Figure 57). 
The Pathogenesis of Ewing Sarcoma/ Results 
Discussion 
The Pathogenesis of Ewing Sarcoma |Discussion 


\section{The role of the MSC axis in ES biology. Targeting CD99 in MSC}

Extensive evidence suggested MSC as the most plausible cell of origin of ES, thus stressing the need for a deeper study of BM-MSC derived from ES patients. Clinically, ES diagnosis is based not only on morphology, but also on the molecular characterization through the presence of the EWSR1-ETS fusions and cell surface overexpression of CD99.[125]

Therefore, the primary aim of our study was to look for early events in MSC-P, such as the presence of EWSR1-ETS fusions and cellular phenotypes that could be exploited to improve therapy or prevention. In order to achieve this, we firstly compared MSC-P with MSC-HD.

Specifically, we observed that MSC-P lack EWSR1-FLI1 fusion gene and EWSR1 rearrangements. This fact may not be surprising, however, in the context of gene fusion-driven neoplasms, it represents an important result of our work, since previous studies performed in Leukemia described that fusion of the MLL (HRX, ALL-1) gene with its partners, initially arises in prenatal myeloid cells, pre-malignant, which after stages of progression during development result in fully malignant leukemic-cells in the adult.[126]Herein, in contrast, we were unable to find EWSR1 rearrangements in cells other than ES cells.

Subsequently, also in chapter 1, we analyzed the presence of other ES surface markers. Here, our goal was to search for possible events occurring prior to the translocation that could enable a permissive environment for further transformation and thus help to solve the causality dilemma of what came first, the EWSR1 fusion or secondary alterations, the chicken or the egg?[35]

However, results described in chapter 1 suggest that immunophenotypic profiles of MSC-P and MSC-HD, are similar regarding ES surface markers 
CD99, CD271, CD54 and CD117. Notwithstanding, our study also showed that ES cells present a few MSC features such as the expression of CD90 and the lack of the hematopoietic markers. Absence of CD34 expression in these ES cells is also an interesting fact, considering that immunotherapy with autologous stem cell transplantation has already been considered in clinical trials for ES.[8, 127] Our results discard the possibility of contamination of the CD34+ niche with tumor cells in auto transplantation.

Altogether we can't discard the possibility of a cellular hierarchy starting in MSC expressing CD99, followed by one or several cellular pre-malignant stages presenting EWSR1 gene rearrangements and further gene deregulation, gradually losing MSC phenotypical characteristics and finally culminating in a fully malignant ES cell bearing the EWSR1 fusion, overexpressing CD99 while maintaining some MSC phenotypical characteristics. (Representation on figure 58).

Since we observed that MSC also express CD99, and taking into account that CD99 is a promising target for innovative therapies in ES, we felt the need to study possible cytotoxic effects of anti-CD99 therapies on MSC. CD99 is overexpressed in over $99 \%$ of ES cells and has been considered as a key causative of ES malignancy and a potential therapeutic target.[37, 86, 125] Contradictory data suggest that if, on the one hand, induced expression of EWSR1-FLI1 upregulates CD99, on the other hand, changes on CD99 expression have not been reported when EWSR1-FLI1 was knocked down in ES cells. $[17,23,70]$ These observations may be reconciled by our finding that MSC show variable baseline expression levels of CD99. 
Despite extensive research, the physiological functionality of CD99 remains unknown. Nevertheless, CD99 knockdown in ES cells induced their neural differentiation and reduced cell malignancy.[70]

Altogether, our previous data confirm that both EWSR1-FLI1 and CD99 are absolutely critical for ES oncogenic phenotype maintenance.[70] In addition, in ES, engagement of CD99 with antibodies resulted in apoptosis of ES cells.[66, 70, 72, 76] Herein, we observed that MSC displaying similar CD99 expression levels to ES cells were unable to undergo apoptosis when exposed to 0662 . This finding is of particular interest in terms of future clinical application of CD99 targeted therapies, since it sustains the limited toxicity and potentially high specificity of this therapeutic approach in ES patients.

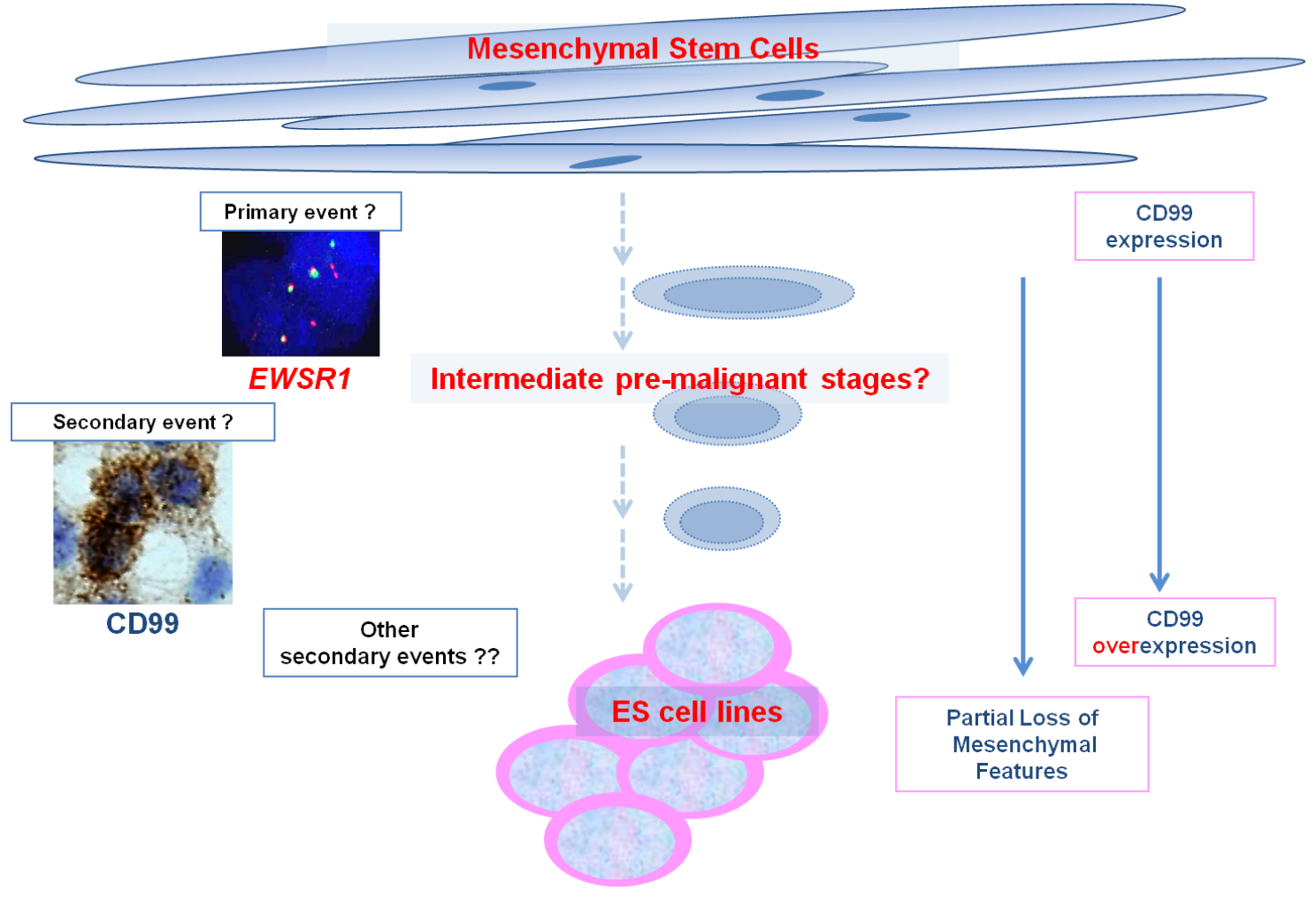

Figure 58. Hypothetic malignant process: from hMSC to pre-malignant stages and fully malignant ES cells. 


\section{The establishment of innovative therapies in ES treatment: outcome from the in vitro and in vivo studies}

\subsection{The relevance of Trabectedin, Zalypsis y Lurbinectedin in ES therapy: outcome from in vitro studies}

The marine derived compound Trabectedin has shown antitumoral activity towards several types of cancer. It has been largely demonstrated that advanced soft-tissue sarcomas are particularly sensitive to this agent. In line with previous studies, in chapter 2 we show that Trabectedin has a powerful anti-proliferative activity towards a large panel of ES cell lines, with no correlation with both the status of p53 and the type of chromosomal fusion harbored by each cell line. Therefore, this urged the need to explore novel related compounds/analogues with different pharmacokinetics. [128]

Zalypsis is a new semi-synthetic tetrahydroisoquinoline alkaloid with demonstrated anti-proliferative activity towards hematological malignancies and several cancer cell lines including the TC71 ES cell line $[54,55,57]$. This agent is currently engaged in phase II clinical trials $[57,58]$. Regarding Lurbinectedin, recent evidence suggests that this molecule has a potent antitumoral activity towards several cancer cell lines such as lung cancer, colorectal cancer or breast cancer. This compound is in phase II clinical development for the treatment of solid tumors. [56]

This group of compounds presents a basic common structure responsible for DNA recognition and binding, while an additional part of the molecule is projected out of the minor groove. [55] For Trabectedin is a tetrahydroisoquinoline; Lurbinectedin presents a tetrahydro- $\beta$-carboline and Zalypsis a trifluorocinammic acid unit. 
In our study, Trabectedin, Zalypsis and Lurbinectidin showed, similar levels of proliferation inhibition and apoptosis induction. These agents showed a potent capacity to induce DNA damage through DSB during short-term drug exposure, and particularly irreparable DSB observed after drug washout. This fact corroborates previous data suggesting that also Zalypsis and Lurbinectedin are potent DSB inductors in ES cell lines and platinum-resistant cells, respectively. [54, 109] Posterior to DSB induction, signaling and repair machinery pathways are rapidly activated. Here, we evaluated the activation of several repair mechanisms through LDMA and protein expression.

We studied activation of DDR after low and high dosage drug exposure in order to determine possible differences between these drugs. Interestingly, a recent publication by Romano $M$ and colleagues revealed that there were in fact slight differences in terms of DDR dependency between these agents, nonetheless their anti-proliferative activity in vitro and in vivo was mostly comparable. This study was performed in several cell lines of sarcomas, however it did not include ES. These studies on DDR dependency were performed on complex models of HR/NER/NHEJ knockout genes.[129] Here, we observed a major deregulation of the DDR after treatment with Trabectedin, with especial overexpression of HR components.

Our results in ES are in agreement with those reported in other tumor models $[129,130]$. Most importantly, we observed a strong deregulation of the DDR after treatment with all agents which associated to their potent in vitro activity may suggest that the combination between these agents and other DNA damage related compounds could be of potential interest. 
Moreover, resistance to Trabectedin has been related to the over expression of the IGF1R pathway, together with the over expression of the efflux bomb Glycoprotein-P.[47] In our study, we observed that cells resistant to Trabectedin are also resistant to Zalypsis and Lurbinectedin, using the TC-ET resistant cell line as a study model. Furthermore, EWSR1-FLI1 inhibition increases cells sensitivity to the action of IGF1R inhibitors, hence, these data suggest that the combination between Trabectedin and IGF1R inhibitors could also represent potential interest in the clinic.[17]

\subsection{The combination of the dual inhibitor Linsitinib with Trabectedin}

In the clinic, Trabectedin failed to demonstrate activity as a single agent against pediatric sarcomas, including ES.[128] Despite this fact, and given that Trabectedin has demonstrated specificity towards ES cells bearing EWSR1-FLI translocations, we found the need to study the combination of this agent with agents such as IGF1R inhibitors.

In chapter 3, we provide in vitro and in vivo evidence that Trabectedin may be advantageously used in combination with inhibitors of the IGF system. Particularly, we provide a rationale for the use of this combination, showing that Trabectedin is able to increase the expression of IGF1R by enhancing the EWSR1-FLI1 occupancy of the IGF1R promoter. EWSR1-FLI1 is the key molecular feature that drives the malignant phenotype in ES cells. [131] It is a hybrid transcription factor, generated by the $t(11 ; 22)(q 24 ; q 12)$ chromosomal translocation, acting either as a transcriptional activator and a transcriptional repressor in ES. [33] Importantly, both the activating and repressive functions of EWSR1-FLI1 are absolutely required for its oncogenic functions [132]. 
Trabectedin was previously found to interfere with the activity of EWSR1FLI1, reversing the expression of the EWSR1-FLI1 induced gene signature and blocking the promoter activity and expression of critical EWSR1-FLI1 downstream targets.[133] These effects likely cooperate with DNA-damage mechanisms of action of Trabectedin, and a combination with SN38 (the active metabolite of irinotecan) was recently proposed to develop a therapy that augments the suppression of EWSR1-FLI1 activity-. [133]. However, here we show that these inhibitory effects induced by Trabectedin, although probably prevalent, are not exclusive. In fact, while we could confirm that both Trabectedin and DXR are able to strongly suppress the binding of EWSR1-FLI1 (both type 1 and type 2) to two target genes (TGF $\beta R 2$ and CD99) both in vitro and in vivo, a significant enhancement of the EWSR1-FLI1 occupancy on the IGF1R promoter was observed only after exposure to Trabectedin.

Prior studies have demonstrated that other DNA binding agents, including mithramycin, and actinomycin D, reduced expression of EWSR1-FLI1 downstream targets and displayed differential specificity, likely due to preferential sequence binding affinities- $[49,134]$ This discovery introduces a certain level of specificity in the action of conventional agents, which is potentially very interesting, but requires further studies since the effects may vary in relation to drugs, transcription factors and cellular context. For example, DXR did not affect the binding of FUS-CHOP to target promoters in myxoid liposarcoma, whereas it was shown here to inhibit the occupancy of EWSR1FLI1 on TGF $\beta$ R2 and CD99 promoters, indicating differences among protein chimeras and drug action in different cellular context. By reporting also an increase and not just a suppression of EWSR1-FLI1 binding to specific target 
promoters we introduced another variable that deserves more ample investigation. In the specific context of ES, the increase in IGF1R expression is absolutely reasonable from a biological point of view considering the importance that IGF system has in the maintenance of ES malignancy [77, 135] and was indeed confirmed also in cells made resistant to Trabectedin. [47]

From a clinical point of view, the increased expression of IGF1R in response to in vitro and in vivo exposure to Trabectedin, provides the rationale for a combined use of Trabectedin with anti-IGF1R agents. We demonstrate here the advantages of this combination either using the HAb AVE1642, which binds the human IGF1R specifically and with high affinity, or the dual inhibitor IGF1R/IR, Linsitinib. AVE1642 is well tolerated as a single agent as well as when combined with docetaxel, gemcitabine/ erlotinib, or DXR, while it achieves promising activity (stabilization $>12$ weeks in around $40 \%$ of patients) in patients with advanced solid tumors. [90, 136] However, clinical studies have clearly shown that only around $10-15 \%$ of ES patients are responsive to anti-IGF-1R antibodies as single agent. [95, 137] This may be very likely due to the concomitant expression of IR-A, besides IGF1R, in ES samples [89], which allows the rapid adaptation of cells to the IGF1R blockade. Based on this evidence the use of a dual IGF1R inhibitor such as Linsitinib should be preferable. This small molecule was found to have antitumoral activity against several tumors [138, 139], including osteosarcoma [140] and was helpful against cells resistant to different agents. [141, 142]

The association of Linsitinib with Trabectedin gave synergistic effects in all 14 ES cell lines here studied, including cells resistant to Trabectedin or to anti-IGF1R agents. This appears to be due mainly to the complementary pro- 
apoptotic effects of the two drugs which by affecting different pathways give rise to a combination able to deliver cell death messages in all ES cells, independently from the status of p53. While treatment with IGF1R antagonists is known to lead to down-regulation of proteins involved in cell survival and inhibition of cell death, thereby recovering cell sensitivity to apoptosis, Trabectedin has been previously described as a potent DNA damaging agent. $[123,138]$ Tavecchio et al. clearly showed that DSBs are not directly caused by the drug, but are formed during the processing/repair of the drug, requiring a functional HR pathway. [107] In addition, Trabectedin also poisons the mechanisms of DDR through the formation of ternary NER proteins-DNATrabectedin complexes (for a review see [143]).

Our results demonstrated that in ES cells Trabectedin increases the expression of BRCA1, BRCA2, key proteins in the HR pathway as well as of XRCC1, which is involved in SSR pathway, resulting in DNA damage as indicated by the phosphorylation of histone $\mathrm{H} 2 \mathrm{AX}$ and accumulation of intranuclear foci. The drug combination with Linsitinib maintains and even increases the up-regulation of members of HR (RAD52, BRCA1 and BRCA2), NER (XPA, ERCC1), and SSR (XRCC1) pathways but also induces a strong downregulation of XRCC4 and XRCC6 as well of MSH4 and MSH5, two molecules involved in the maintenance of genomic stability and mitotic DSB repair, indicating general alterations of DNA damage responses and repair pathways. [144] This is in line with the recent evidence that IGF-1R inhibition induces a direct functional defect in DSB repair by both NHEJ and HR, besides indirectly impairing HR through influences on the expression and/or activation of cell cycle regulators. [145] 
The importance of Trabectedin and IGF system inhibitors in DNA damage response and repair pathways, which have implications for the therapeutic efficacy and potential toxicity of this combined therapy in the clinic, merits further research to better elucidate the molecular mechanisms and protein interactions.

Overall, we provide the rationale for combining Trabectedin to anti-IGF$1 \mathrm{R}$ inhibitors. We showed that Trabectedin enhances the binding of EWSR1FLI1 to target genes. Specifically, IGF-1R expression is activated after treatment with Trabectedin and anti-IGF1R agents improving efficacy of Trabectedin in cell lines and xenografts. We thus propose the use of a clinically relevant combination therapy that may exploit the complementary mechanisms of action of the two drugs.

\subsection{PARPi in ES. The combination of Olaparib and Trabectedin}

The role played by PARP1 in ES as a transcriptional regulator of EWSR1-FLI1 has triggered an enormous interest for testing PARPi in this tumor.[102, 103] PARPi also disrupts the DDR system of tumor cells.[98, 101] Therefore we formulated the hypothesis that the combination of Trabectedin with a PARPi, able to inhibit EWSR1-FLI1 and also interfere with the DDR system, could be of enormous interest as a therapeutic strategy. The results obtained in chapter 4 support the use of a combination of PARPi \&Trabectedin in ES.

The relationship between PARP1 and ES has long been known.[146] However, the knowledge of the more specific relevant role played by this protein in ES is much more recent.[102, 103] Brenner et al described that PARP1 interacts physically with EWSR1-FLI1 fusion, acting in a positive feed-back as a 
cofactor regulating its own expression.[102] In a retrospective study, the gain of the long arm of chromosome 1 was revealed to be of prognostic value in ES.[10] In the expression profiling studies, PARP1 was up-regulated, being clearly one of the most interesting genes to be studied.[10]

The mechanism of action of PARPi in cancer cells appears to be related mainly with DNA damage induction, by the formation of irreparable SSB. These drugs are used in monotherapy in tumors with specific molecular alterations in the DDR system, such as BRCA1/BRCA2.[147, 148]

The use of PARPi in monotherapy in breast cancer to treat tumors that are defective in BRCA1 and/or BRCA2 is, in fact, the first example of synthetic lethality.[147, 148] So far, there is no evidence of BRCA1/BRCA2 mutations in ES. However, the use of PARPi has been expanded to tumors harboring ETS translocations such as prostate cancer and ES.[102, 104] In ES particularly, sensitivity to PARPi seems to be related with the deregulation of EWSR1-FLI1 transcriptional activity of its target genes.[102, 103] Additionally, in these tumors, EWSR1-FLI1 induces DNA damage per se in ES cells.[102]

Olaparib was the drug chosen in most of the studies performed in ES using PARPi.[102, 103, 149] In the present study, we decided to study the efficacy of three different PARPi. All of them have been largely studied in solid tumors, but to the best of our knowledge, Veliparib and Iniparib, have never been tested before in ES. Olaparib and Veliparib have a similar mechanism of action in which they compete with NAD to bind to the catalytic active site of PARP enzyme. On the contrary, Iniparib (BSI-201) does not compete with the active site of PARP enzyme but induces an irreversible modification of PARP1 and stimulates a PARP1-protease.[100] This drug entered in a phase III study in 
triple-negative breast cancer patients; however its efficacy and specificity has been recently questioned.[150] According to our study, Iniparib was by far the less active of the three PARPi. By contrast, Olaparib was the most active compound against $\mathrm{ES}$ cell lines, with $\mathrm{IC}_{50}$ of proliferation ranging from the high nanomolar to the low micromolar range, which is very similar to data published by other groups.[103] Interestingly, in contrast to previous works,[103] Olaparib was not only a potent cytotoxic agent after 3 days of drug exposure, but its action lasted at least 6 days, until when the $\mathrm{IC}_{50}$ suffered a significant decrease in all cell lines assayed. However, in the in vivo study, Olaparib alone was only partially effective in terms of tumor shrinkage. This is in accordance with previous studies, where Olaparib was not able to halt the tumor growth.[102] In fact, a recent CT with Olaparib in ES patients was withdrawn due to absence of objective responses (Revised in [86] ).

Therefore, and since PARPi are not only used in monotherapy but in combination with radiotherapy and other chemotherapeutic agents which provoke DNA damage, we decided to combine Olaparib with Trabectedin. Interestingly, Grohar and colleagues recently suggested combining Trabectedin with drugs that further increase DNA damage and diminish the EWSR1-FLI1 transcriptional activity. Grohar et al demonstrated that the combination of Trabectedin and Irinotecan in ES showed a synergistic activity confirming the molecular precision treatment concept.[133, 151] Precision medicine could also be applied to the combination of PARPi and Trabectedin. In fact, PARPi, and particularly, Olaparib, are involved in DNA damage and alterations of the EWSR1-FLI1 transcriptional activity. Our results show that the combination of Trabectedin and Olaparib was highly synergistic, increasing the apoptotic 
activity, arresting cell cycle at G2/M phases, and reducing the cell invasion of ES cells. This antitumoral activity was also observed in vivo, where the drug combination deeply reduced tumor growth, even when Trabectedin was administered one week after Olaparib.

This phenomenon is very interesting because no appreciable toxicities in animals were observed, which could be of interest in clinic. Furthermore, we observed that although both agents alone induced DNA damage through accumulation of $\mathrm{yH} 2 \mathrm{AX}$ foci and deregulation of the DDR pathways in vitro, the combination dramatically increased this phenomenon. This drug combination actively deregulated the major DDR pathways. The effects of drug combination in vivo confirmed the in vitro data, increasing accumulation of $\mathrm{yH} 2 \mathrm{AX}$ foci with overexpression of BRCA2.

The main conclusion from this study is that the combination of Trabectedin and Olaparib substantially reduced tumor growth compared to each single treatment, even when low dosages of Trabectedin were administered. Other studies have combined Olaparib with drugs/strategies inducing DNA damage, namely Temozolamide and radiotherapy.[102, 149] In both cases the combination regimen showed a reduction of tumor volume when compared to the control situation. However, DNA damage induction and DDR activation machinery was not deeply studied. Here, we not only observed that this combination works both in vitro and in vivo but we also report the deregulation of the major involved DDR pathways. Trabectedin might represent a better option than Temozolamide or radiotherapy since this agent also disrupts EWSR1-FLI1 activity.[49] 


\section{Final Outcome and future perspectives}

In this work we studied the importance of targets such as CD99, the fusion protein, the IGF1-IGF1R/IGF2-IR system and PARP1. These have been described as major role playing parts in ES malignancy.

In chapter 1, we have described that MSCs showed CD99 expression, However, in contrast to what was observed in ES cells, MSCs are not sensitive to anti-CD99. Regarding the application of targeted anti-CD99 therapies in the clinic, this result represents an interesting development given that MSC would not be affected by the treatment, and tumor cells would be strongly affected due to their overexpression of CD99. Nevertheless, other cell compartments also express CD99, namely leukocytes, and should also be studied in terms of induced toxicity. Also, results from chapter 1 unveiled new possible therapeutic targets, such as endoglin (CD105). Endoglin is overexpressed in MSC and our studies revealed that also ES cells show expression of CD105. In fact, very recent evidence suggested that the inhibition of CD105 in ES cell lines resulted in reduced invasiveness and malignancy. Endoglin expression in ES tumor samples was correlated with bad prognosis.[152]

In chapter 2 we describe that Trabectedin, a new marine compound highly active in vitro towards sarcomas bearing translocations exerts its action by inducing irreparable DNA damage that leads to S-phase accumulation and cell death through apoptosis induction. Its related compounds, Zalypsis and Lurbinectedin, also showed high cytotoxic activity towards ES cells. Zalypsis was highly toxic, however it appears to have a different mechanism of action, in contrast to Lurbinectedin which similarly to Trabectedin disrupts the major DDR pathways responsible for DSB repair. 
Given these results, we continued our study by combining Trabectedin with two new agents: i) anti-IGF1R; ii) PARPi.

In chapter 3, we described that Trabectedin strongly deregulates the transactivation activity of EWSR1-FLI1 fusion protein and surprisingly, results in the overexpression of IGF1R. In fact, combinations of Trabectedin with MAb anti-IGF1R were highly active in in vitro and in vivo models. Moreover, and taking into account that the mechanism of resistance to Trabectedin is related to the overexpression of the IGF1R axis and the switch from IGF1-IGF1R to IGF2IR dependency, the combination of Trabectedin and dual inhibitor Linsitinib appeared to be strongly appealing. In fact, we describe that this combination results in strong synergism in ES cell lines, inducing apoptosis, cell cycle arrest and also an improvement on the DNA damaging action of Trabectedin alone.

Finally, on chapter 4 we studied the sensitivity of ES cells to PARPi, and its relation with the previously defined 1qG. We describe that Olaparib is the most toxic PARPi agent tested in this study. When combined with Trabectedin, in vitro and in vivo data revealed that this combination results in highly synergistic effects and is dependent on the DNA damaging action of both agents.

The IGF1-IGF1R pathway can be targeted with small molecules such as ADW742, HSP90 inhibitors, mTOR inhibitors or endocytosis inhibitors, or MAb irreversibly blocking IGF1R. The new small molecule Linsitinib is a dual inhibitor able to inhibit both IGF1R and IR, thus overcoming the mechanism of resistance adapted by ES cells to IGF1R inhibitory therapies. CD99 is also an attractive target. Moreover, therapies targeting the DNA, either through DNA damage induction (Trabectedin), dysregulation of the fusion protein 
transcriptional activity (Trabectedin, YK-4-279. ARA-c, mithramycin, PARPi), inhibition of mitosis (Zoledronic Acid) and epigenetic therapies (HDACi and demethylating agent 5-AZA) have also prove to have strong toxic activity either in vitro or in vivo (Figure 59).

Altogether, data from these studies generate novel combinative approaches that could be adapted to the clinic, giving rise to new effective therapeutic strategies in ES patients. 


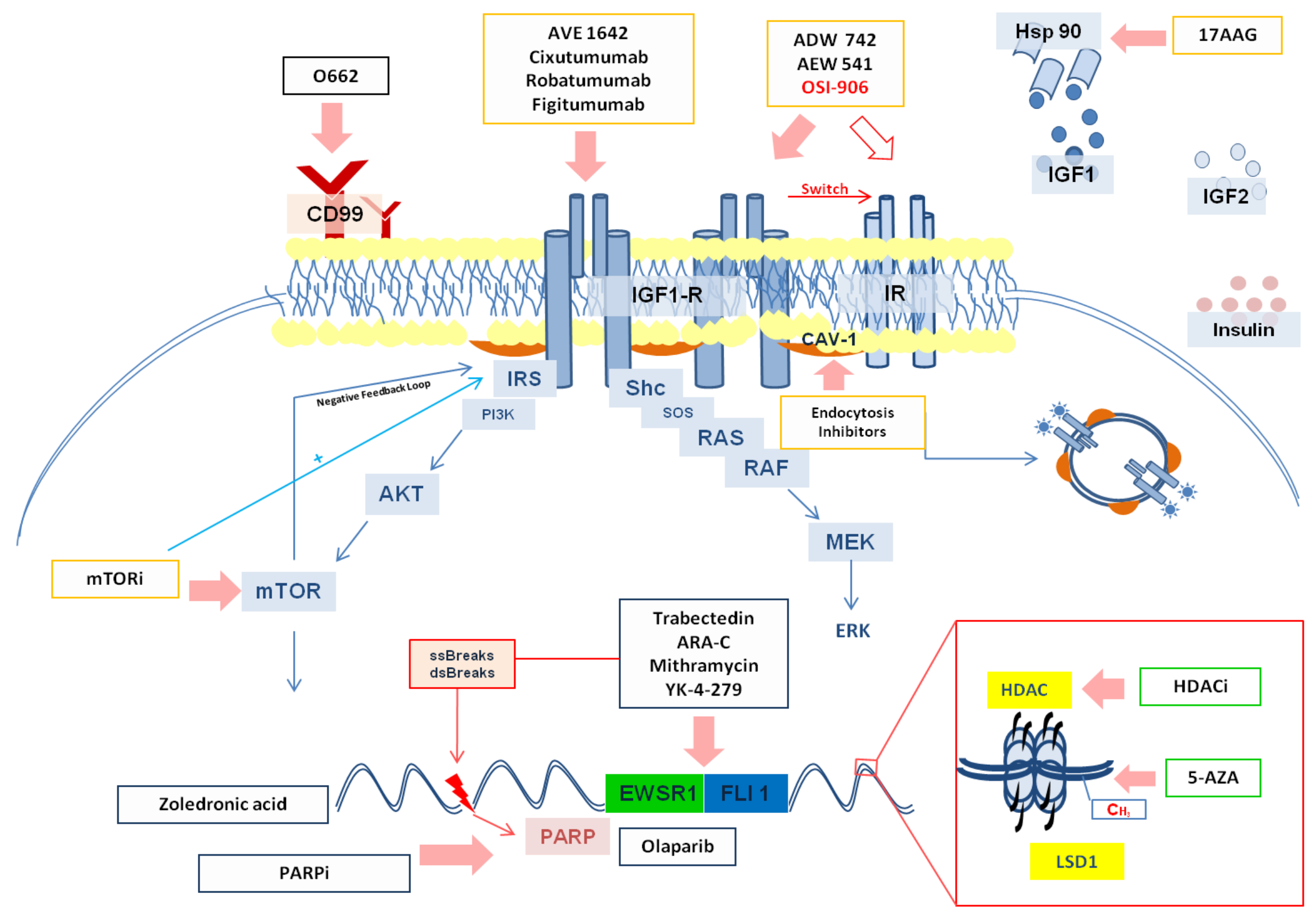


Figure 59. Complex pathways of action of different drugs on ES cells. The IGF1-IGF1R pathway can be targeted with small molecules such as ADW742, HSP90 inhibitors, mTOR inhibitors or endocytosis inhibitors, or MAb irreversibly blocking IGF1R. The new small molecule Linsitinib is a dual inhibitor able to inhibit both IGF1R and IR, thus overcoming the mechanism of resistance adapted by ES cells to IGF1R inhibitory therapies. CD99 is also an attractive target. Moreover, therapies targeting the DNA, either through DNA damage induction (Trabectedin), dysregulation of the fusion protein transcriptional activity (Trabectedin, YK-4-279. ARA-c, mithramycin, PARPi), inhibition of mytosis (Zoledronic Acid) and epigenetic therapies (HDACi and demethylating agent 5-AZA) have also prove to have strong toxic activity either in vitro or in vivo. 
Conclusions 
The Pathogenesis of Ewing Sarcoma / Conclusions 


\section{Conclusions}

From this work we can assess the following conclusions:

1. MSC-EP present a similar phenotypic profile when compared to $\mathrm{MSCH}$ HD regarding CD99, CD271, CD117 ES pathogenic markers. ES Cell lines and ES tumor samples present overlapping features with MSC, such as overexpression of CD90 and CD105, and absence of CD34 and CD45 expression. CD99 is overexpressed in ES cell lines and tumor samples with respect to MSC. MSC derived from healthy tissues heterogeneously express CD99. CD99 inhibition impairs ES cell viability but not that of MSC. Targeting CD99 could then be exploited as a safe therapeutic strategy, targeting specifically CD99 overexpressing cells.

2. Trabectedin and Trabectedin-related compounds are highly toxic to ES cells. Trabectedin appears to be slightly more active in terms of proliferation inhibition and global cell death related mechanisms than its related compounds. All agents induce DSB, however, in contrast to Zalypsis, Trabectedin and Lurbinectedin strongly deregulate DDR mechanisms.

3. Trabectedin deregulates the transcriptional activity of the fusion protein EWSR1-FLI1. Treatment with Trabectedin leads to an up-regulation of IGF1R in ES cells. The IGF1R-IR dual inhibitor Linsitinib is toxic towards ES cells. Treatment with Linsitinb results in cell cycle arrest, proliferation inhibition and apoptosis induction. The combination of Linsitinb and Trabectedin is synergistic in ES cell lines and leads to an increase on 
DNA damage and higher deregulation of DDR pathways when compared to treatment with Trabectedin alone.

4. Among a group of PARPi including Olaparib Veliparib and Iniparib, Olaparib is more toxic to ES cells than the other two, independently of PARP1 expression and 1qG. Treatment with Olaparib induces cell death by apoptosis and G2/M cell cycle accumulation. Combination of Trabectedin and Olaparib resulted in highly synergistic effects in most of the ES cell lines studied. These synergistic effects were validated in vitro and in a xenograft model of ES with a significant reduction of tumor growth. The mechanism behind tumor arrest is DNA damage induction, as observed by the presence of $\mathrm{\gamma H} 2 \mathrm{AX}$ foci and further overexpression of DDR protein BRCA2. 
Appendix 
The Pathogenesis of Ewing Sarcoma / Appendix 
Table XXVI.S. Fold Change in the TC71 cell line after treatment with 500pM pf Trabectedin, Zalypsis and Lurbinectedin

\begin{tabular}{|c|c|c|c|c|c|}
\hline \multirow[b]{2}{*}{500 pM } & \multicolumn{4}{|c|}{$\begin{array}{c}\text { Fold Change } \\
\text { (comparing to control group) }\end{array}$} & \multirow[b]{2}{*}{ Unigene } \\
\hline & Trabectedin & Zalypsis & PM00113 & Lurbinectidin & \\
\hline Apex1 & 0.8661 & 0.1363 & 0.882 & 0.8357 & Mm.203 \\
\hline Apex2 & 1.6808 & 0.0941 & 2.341 & 0.8647 & Mm.440275 \\
\hline Atm & 1.1977 & 0.119 & 0.6266 & 0.958 & Mm.5088 \\
\hline Atr & 1.1446 & 0.1084 & 0.6703 & 0.8474 & Mm.212462 \\
\hline Atxn3 & 1.6724 & 0.1369 & 1.1826 & 0.8018 & Mm.485508 \\
\hline Brca1 & 2.0554 & 0.1363 & 1.3633 & 0.9662 & Mm.244975 \\
\hline Brca2 & 1.7784 & 0.1128 & 1.245 & 0.728 & Mm.236256 \\
\hline Brip1 & 1.6626 & 0.11 & 1.2711 & 1.0515 & Mm.186143 \\
\hline Ccnh & 0.9433 & 0.1343 & 0.495 & 0.6892 & Mm.18474 \\
\hline Ccno & 1.3788 & 0.1353 & 1.223 & 0.8412 & Mm.25457 \\
\hline Cdk7 & 0.855 & 0.1179 & 0.5738 & 0.6588 & Mm.259718 \\
\hline $\mathrm{Ddb} 1$ & 0.741 & 0.0788 & 0.9046 & 0.4273 & Mm.289915 \\
\hline Ddb2 & 1.3107 & 0.1709 & 1.3215 & 1.0538 & Mm.389334 \\
\hline Dmc1 & 0.8487 & 0.0735 & 0.6042 & 0.4769 & Mm.2524 \\
\hline Ercc1 & 1.6165 & 0.1269 & 1.6262 & 0.9327 & Mm.280913 \\
\hline Ercc2 & 1.5127 & 0.0986 & 1.257 & 0.8831 & Mm.36524 \\
\hline Ercc3 & 1.4545 & 0.0885 & 1.2837 & 0.8845 & Mm.282335 \\
\hline Ercc4 & 1.1188 & 0.0943 & 0.911 & 0.7395 & Mm.287837 \\
\hline Ercc5 & 1.2683 & 0.155 & 0.6649 & 0.8784 & Mm.2213 \\
\hline Ercc6 & 1.235 & 0.0893 & 0.9963 & 0.8343 & Mm.318310 \\
\hline Ercc8 & 0.9503 & 0.1524 & 0.6788 & 0.7493 & Mm.212208 \\
\hline Exo1 & 1.4751 & 0.1026 & 1.0627 & 1.133 & Mm.283046 \\
\hline Fen1 & 0.7348 & 0.1105 & 0.5561 & 0.8244 & Mm.2952 \\
\hline Lig1 & 0.9255 & 0.1278 & 1.051 & 0.6702 & Mm.288179 \\
\hline Lig3 & 0.789 & 0.1971 & 1.0163 & 0.6386 & Mm.277136 \\
\hline Lig4 & 0.9069 & 0.1813 & 0.7341 & 0.6587 & Mm.80584 \\
\hline Mgmt & 0.8214 & 0.1953 & 0.6593 & 0.523 & Mm.440219 \\
\hline Mlh1 & 1.1945 & 0.1569 & 0.7785 & 0.7322 & Mm.196006 \\
\hline Mlh3 & 1.5126 & 0.1677 & 1.0911 & 0.8813 & Mm.311981 \\
\hline Mms19 & 1.4442 & 0.1585 & 1.1665 & 0.9182 & Mm.218940 \\
\hline Mpg & 1.4038 & 0.2188 & 0.9063 & 0.9264 & Mm.263161 \\
\hline Mre11a & 1.2778 & 0.1233 & 0.8745 & 0.7876 & Mm.149071 \\
\hline Msh2 & 1.1348 & 0.3004 & 0.6582 & 1.1107 & Mm.4619 \\
\hline Msh3 & 0.8824 & 0.1172 & 0.7409 & 0.7067 & Mm.343101 \\
\hline
\end{tabular}


The Pathogenesis of Ewing Sarcoma / Appendix

\begin{tabular}{|c|c|c|c|c|c|}
\hline Msh4 & 0.2078 & 0.2273 & 0.4561 & 0.4273 & Mm.272226 \\
\hline Msh5 & 1.1072 & 0.2879 & 1.3294 & 0.8396 & Mm.24192 \\
\hline Msh6 & 1.1016 & 0.4557 & 1.1519 & 0.7913 & Mm.18210 \\
\hline Mutyh & 1.5132 & 0.5019 & 1.2867 & 1.0334 & Mm.180333 \\
\hline Neil1 & 1.9522 & 0.5275 & 1.4544 & 0.8308 & Mm.35749 \\
\hline Neil2 & 1.0011 & 0.2602 & 1.1079 & 0.663 & Mm.239490 \\
\hline Neil3 & 1.4211 & 0.3174 & 0.9461 & 0.7834 & Mm.281749 \\
\hline Nthl1 & 1.1052 & 0.234 & 0.6937 & 0.756 & Mm.148315 \\
\hline Ogg1 & 1.4257 & 0.3876 & 1.1404 & 1.0052 & Mm.43612 \\
\hline Parp1 & 1.024 & 0.1574 & 1.0434 & 0.7041 & Mm.277779 \\
\hline Parp2 & 1.3128 & 0.5383 & 1.0355 & 0.9791 & Mm.281482 \\
\hline Parp3 & 1.3464 & 0.1872 & 1.5107 & 0.9139 & Mm.273659 \\
\hline Pms1 & 0.8478 & 0.333 & 0.5003 & 0.8039 & Mm.60499 \\
\hline Pms2 & 0.876 & 0.3639 & 1.2586 & 0.574 & Mm.2950 \\
\hline Pnkp & 1.109 & 0.6533 & 1.0208 & 1.0239 & Mm.238254 \\
\hline Polb & 1.1693 & 0.7468 & 0.997 & 1.0364 & Mm.123211 \\
\hline Pold3 & 1.7535 & 0.7021 & 1.5677 & 0.932 & Mm.37562 \\
\hline Poll & 1.364 & 0.5395 & 1.5999 & 0.6789 & Mm.46509 \\
\hline Prkdc & 1.5095 & 0.4719 & 0.6305 & 0.9746 & Mm.71 \\
\hline Rad18 & 1.4588 & 0.4074 & 1.0661 & 0.8245 & Mm.103812 \\
\hline Rad21 & 1.213 & 0.5552 & 1.011 & 0.8521 & Mm.182628 \\
\hline Rad23a & 1.3362 & 0.3123 & 0.9198 & 0.6888 & Mm.477498 \\
\hline Rad23b & 1.0545 & 0.4414 & 0.8704 & 0.5845 & Mm.196846 \\
\hline Rad50 & 0.9753 & 0.2667 & 0.6704 & 0.6965 & Mm.4888 \\
\hline Rad51 & 1.2228 & 0.5088 & 0.9504 & 0.6969 & Mm.471596 \\
\hline Rad51c & 0.3695 & 0.2687 & 0.4508 & 0.368 & Mm.37376 \\
\hline Rad51l1 & 1.0815 & 1.1088 & 1.1045 & 0.8669 & Mm.341756 \\
\hline Rad51l3 & 1.023 & 0.8496 & 0.8768 & 1.103 & Mm.9286 \\
\hline Rad52 & 1.4097 & 0.7543 & 1.2749 & 0.724 & Mm.149 \\
\hline Rad54l & 2.0272 & 1.1258 & 1.7353 & 1.2883 & Mm.3655 \\
\hline Rfc1 & 1.7659 & 0.7676 & 0.9282 & 1.0308 & Mm.148877 \\
\hline Rpa1 & 1.3418 & 0.6971 & 1.0838 & 0.8934 & Mm.180734 \\
\hline Rpa3 & 1.3121 & 0.7563 & 0.641 & 0.9109 & Mm.29073 \\
\hline Slk & 1.2 & 0.4554 & 0.9671 & 0.6649 & Mm.281011 \\
\hline Smug1 & 1.2854 & 0.5451 & 0.9941 & 0.7041 & Mm.254820 \\
\hline Tdg & 1.0025 & 0.4142 & 0.8427 & 0.6846 & Mm.347607 \\
\hline Top3a & 1.2787 & 0.575 & 1.3184 & 0.8075 & Mm.477819 \\
\hline Top3b & 0.8598 & 0.6322 & 1.1736 & 0.7704 & Mm.326089 \\
\hline Trex1 & 1.5505 & 1.4102 & 0.0014 & 1.2262 & Mm.439964 \\
\hline Ung & 0.9389 & 0.9462 & 0.5454 & 0.9806 & Mm.1393 \\
\hline
\end{tabular}


The Pathogenesis of Ewing Sarcoma / Appendix

\begin{tabular}{cccccc}
\hline Xab2 & 1.4133 & 1.1708 & 1.4103 & 1.0421 & $M m .23739$ \\
Xpa & 1.275 & 0.8219 & 0.9456 & 0.7093 & $M m .247036$ \\
Xpc & 1.4458 & 0.8216 & 2.0557 & 0.7343 & Mm.2806 \\
Xrcc1 & 1.6518 & 0.7994 & 1.29 & 0.9979 & Mm.4347 \\
Xrcc2 & 1.6622 & 0.8678 & 1.4798 & 1.1008 & Mm.143767 \\
Xrcc3 & 1.4139 & 0.6154 & 0.9002 & 0.7896 & Mm.19082 \\
Xrcc4 & 0.3671 & 0.3869 & 0.3037 & 0.4946 & Mm.37531 \\
Xrcc5 & 1.0727 & 0.6089 & 1.0139 & 0.8485 & $M m .246952$ \\
Xrcc6 & 0.7035 & 0.5768 & 0.8473 & 0.6619 & $M m .288809$ \\
Xrcc6bp1 & 0.737 & 0.7766 & 1.0094 & 0.834 & $M m .276769$ \\
\hline
\end{tabular}

Table XXVII.S. Fold Change in the TC71 cell line after treatment with 200pM pf Trabectedin, Zalypsis and Lurbinectedin

\begin{tabular}{|c|c|c|c|c|c|}
\hline \multirow[b]{2}{*}{200 pM } & \multicolumn{4}{|c|}{$\begin{array}{c}\text { Fold Change } \\
\text { (comparing to control group) }\end{array}$} & \multirow[b]{2}{*}{ Unigene } \\
\hline & Trabectedin & Zalypsis & PM00113 & Lurbinectidin & \\
\hline APEX1 & 0.7222 & 0.6484 & 0.7262 & 0.789 & Mm.203 \\
\hline APEX2 & 0.74 & 0.6622 & 0.6728 & 0.789 & Mm.440275 \\
\hline ATM & 0.9683 & 0.869 & 0.9029 & 1.0098 & Mm.5088 \\
\hline ATR & 0.8839 & 0.8557 & 0.7726 & 0.8952 & Mm.212462 \\
\hline ATXN3 & 0.8961 & 0.7392 & 0.8012 & 0.9406 & Mm.485508 \\
\hline BRCA1 & 1.0809 & 0.814 & 1.017 & 1.1095 & Mm.244975 \\
\hline BRCA2 & 1.0704 & 0.7321 & 0.8832 & 0.9189 & Mm.236256 \\
\hline BRIP1 & 0.8979 & 0.6913 & 0.7961 & 0.8942 & Mm.186143 \\
\hline $\mathrm{CCNH}$ & 0.8476 & 0.7803 & 0.7832 & 0.8225 & Mm.18474 \\
\hline CCNO & 0.9602 & 0.733 & 1.1404 & 1.0195 & Mm.25457 \\
\hline CDK7 & 0.7556 & 0.7471 & 0.7857 & 0.8381 & Mm.259718 \\
\hline DDB1 & 0.8185 & 0.7033 & 0.8742 & 0.9601 & Mm.289915 \\
\hline DDB2 & 0.9429 & 0.6936 & 0.7774 & 0.8996 & Mm.389334 \\
\hline DMC1 & 0.7567 & 0.706 & 0.7741 & 0.8572 & Mm.2524 \\
\hline ERCC1 & 0.7602 & 0.7257 & 0.987 & 0.744 & Mm.280913 \\
\hline ERCC2 & 0.8175 & 0.7202 & 0.8695 & 0.8747 & Mm.36524 \\
\hline ERCC3 & 0.8781 & 0.6939 & 0.8209 & 0.896 & Mm.282335 \\
\hline ERCC4 & 0.8518 & 0.6383 & 0.7622 & 0.8184 & Mm.287837 \\
\hline ERCC5 & 0.9071 & 0.8354 & 0.8438 & 0.8233 & Mm.2213 \\
\hline ERCC6 & 0.9999 & 0.8094 & 0.7885 & 0.8507 & Mm.318310 \\
\hline ERCC8 & 0.8299 & 0.7567 & 0.7705 & 0.7932 & Mm.212208 \\
\hline
\end{tabular}


The Pathogenesis of Ewing Sarcoma / Appendix

\begin{tabular}{|c|c|c|c|c|c|}
\hline EXO1 & 0.972 & 0.8881 & 0.9181 & 0.9231 & Mm.283046 \\
\hline FEN1 & 0.7376 & 0.7538 & 0.6546 & 0.7804 & Mm.2952 \\
\hline LIG1 & 0.7267 & 0.6611 & 0.9141 & 0.8569 & Mm.288179 \\
\hline LIG3 & 0.8905 & 0.694 & 0.7666 & 1.0447 & Mm.277136 \\
\hline LIG4 & 0.739 & 0.6914 & 0.6573 & 0.6853 & Mm.80584 \\
\hline MGMT & 0.6968 & 0.5676 & 0.5289 & 0.7045 & Mm.440219 \\
\hline MLH1 & 0.7558 & 0.5904 & 0.7067 & 0.7368 & Mm.196006 \\
\hline MLH3 & 0.9086 & 0.7887 & 0.8134 & 0.9449 & Mm.311981 \\
\hline MMS19 & 0.8515 & 0.8415 & 0.8673 & 0.9363 & Mm.218940 \\
\hline MPG & 1.006 & 0.8787 & 1.0051 & 1.0321 & Mm.263161 \\
\hline MRE11A & 0.8667 & 0.7218 & 0.8299 & 0.8549 & Mm.149071 \\
\hline MSH2 & 0.9683 & 0.814 & 0.7951 & 0.9663 & Mm.4619 \\
\hline MSH3 & 0.864 & 0.6934 & 0.7832 & 0.8328 & Mm.343101 \\
\hline MSH4 & 1.3814 & 1.0755 & 0.9983 & 0.246 & Mm.272226 \\
\hline MSH5 & 0.8644 & 0.8074 & 0.9206 & 0.9446 & Mm.24192 \\
\hline MSH6 & 0.7247 & 0.6761 & 0.6153 & 0.7877 & Mm.18210 \\
\hline MUTYH & 0.9435 & 0.6943 & 0.8412 & 0.9698 & Mm.180333 \\
\hline NEIL1 & 0.9797 & 0.8038 & 0.9587 & 1.0031 & Mm.35749 \\
\hline NEIL2 & 0.7566 & 0.7259 & 0.783 & 0.8305 & Mm.239490 \\
\hline NEIL3 & 1.0197 & 0.9123 & 0.9094 & 1.0288 & Mm.281749 \\
\hline NTHL1 & 1.0135 & 0.8516 & 0.932 & 0.8564 & Mm.148315 \\
\hline OGG1 & 0.9951 & 0.8212 & 1.0328 & 0.9954 & Mm.43612 \\
\hline PARP1 & 0.8187 & 0.6978 & 0.825 & 0.7947 & Mm.277779 \\
\hline PARP2 & 1.0099 & 0.9446 & 0.8829 & 0.9352 & Mm.281482 \\
\hline PARP3 & 1.0543 & 0.7538 & 0.9807 & 0.9796 & Mm.273659 \\
\hline PMS1 & 1.1424 & 1.0823 & 1.0123 & 1.1277 & Mm.60499 \\
\hline PMS2 & 0.7923 & 0.7403 & 0.9015 & 0.998 & Mm.2950 \\
\hline PNKP & 0.7729 & 0.7349 & 0.9646 & 0.9567 & Mm.238254 \\
\hline POLB & 0.6204 & 0.6182 & 0.6815 & 0.7013 & Mm.123211 \\
\hline POLD3 & 0.7222 & 0.4806 & 0.6022 & 0.6676 & Mm.37562 \\
\hline POLL & 0.7378 & 0.6094 & 0.9131 & 0.8933 & Mm.46509 \\
\hline PRKDC & 0.8833 & 0.7611 & 0.874 & 0.8913 & Mm.71 \\
\hline RAD18 & 1.066 & 0.8551 & 0.9863 & 1.0289 & Mm.103812 \\
\hline RAD21 & 0.8246 & 0.7031 & 0.8593 & 0.7697 & Mm.182628 \\
\hline RAD23A & 1.149 & 0.9306 & 1.1334 & 1.1097 & Mm.477498 \\
\hline RAD23B & 0.8748 & 0.7455 & 0.7726 & 0.8043 & Mm.196846 \\
\hline RAD50 & 1.0624 & 0.8617 & 0.9413 & 0.9859 & Mm.4888 \\
\hline RAD51 & 1.0461 & 0.8179 & 0.8323 & 0.972 & Mm.471596 \\
\hline RAD51B & 0.5961 & 0.6012 & 0.5971 & 0.7212 & Mm.37376 \\
\hline RAD51C & 0.6742 & 0.6086 & 0.5563 & 0.5818 & Mm.341756 \\
\hline
\end{tabular}


The Pathogenesis of Ewing Sarcoma / Appendix

\begin{tabular}{cccccc}
\hline RAD51D & 0.6065 & 0.55 & 0.6442 & 0.5395 & $M m .9286$ \\
RAD52 & 0.8954 & 0.7493 & 1.1696 & 0.9996 & Mm.149 \\
RAD54L & 0.9255 & 0.8946 & 0.8891 & 1.0494 & Mm.3655 \\
RFC1 & 0.7971 & 0.7453 & 0.8706 & 0.8167 & $\mathrm{Mm} .148877$ \\
RPA1 & 0.9724 & 0.793 & 0.8323 & 0.9354 & $\mathrm{Mm} .180734$ \\
RPA3 & 1.0954 & 1.0408 & 1.0544 & 1.0553 & $\mathrm{Mm} .29073$ \\
SLK & 1.0313 & 0.7609 & 0.8536 & 0.9617 & $\mathrm{Mm} .281011$ \\
SMUG1 & 1.0184 & 0.9345 & 1.0716 & 1.1263 & $\mathrm{Mm} .254820$ \\
TDG & 0.8717 & 0.682 & 0.7613 & 0.8368 & $\mathrm{Mm} .347607$ \\
TOP3A & 1.0541 & 0.8169 & 1.0448 & 1.3144 & $\mathrm{Mm} .477819$ \\
TOP3B & 1.0187 & 0.8685 & 1.1696 & 1.3452 & $\mathrm{Mm} .326089$ \\
TREX1 & 0.9022 & 0.7878 & 0.8352 & 1.0304 & $\mathrm{Mm} .439964$ \\
UNG & 0.5367 & 0.5748 & 0.5788 & 0.672 & $\mathrm{Mm} .1393$ \\
XAB2 & 0.899 & 0.8081 & 0.993 & 0.9529 & $\mathrm{Mm} .23739$ \\
XPA & 1.0265 & 1.0246 & 1.0683 & 1.0417 & $\mathrm{Mm} .247036$ \\
XPC & 0.901 & 0.6318 & 0.8196 & 0.7099 & $\mathrm{Mm} .2806$ \\
XRCC1 & 1.1294 & 0.9136 & 1.1957 & 1.2924 & $\mathrm{Mm} .4347$ \\
XRCC2 & 1.0518 & 0.8628 & 0.8969 & 1.0153 & $\mathrm{Mm} .143767$ \\
XRCC3 & 0.7105 & 0.5634 & 0.79 & 0.7381 & $\mathrm{Mm} .19082$ \\
XRCC4 & 0.7592 & 0.7413 & 0.6515 & 0.8174 & $\mathrm{Mm} .37531$ \\
XRCC5 & 1.055 & 0.8344 & 0.927 & 1.022 & $\mathrm{Mm} .246952$ \\
XRCC6 & 0.8183 & 0.7256 & 0.7191 & 0.7519 & $\mathrm{Mm} .288809$ \\
XRCC6BP1 & 1.062 & 0.9968 & 1.0058 & 0.9687 & $\mathrm{Mm} .276769$ \\
\hline
\end{tabular}



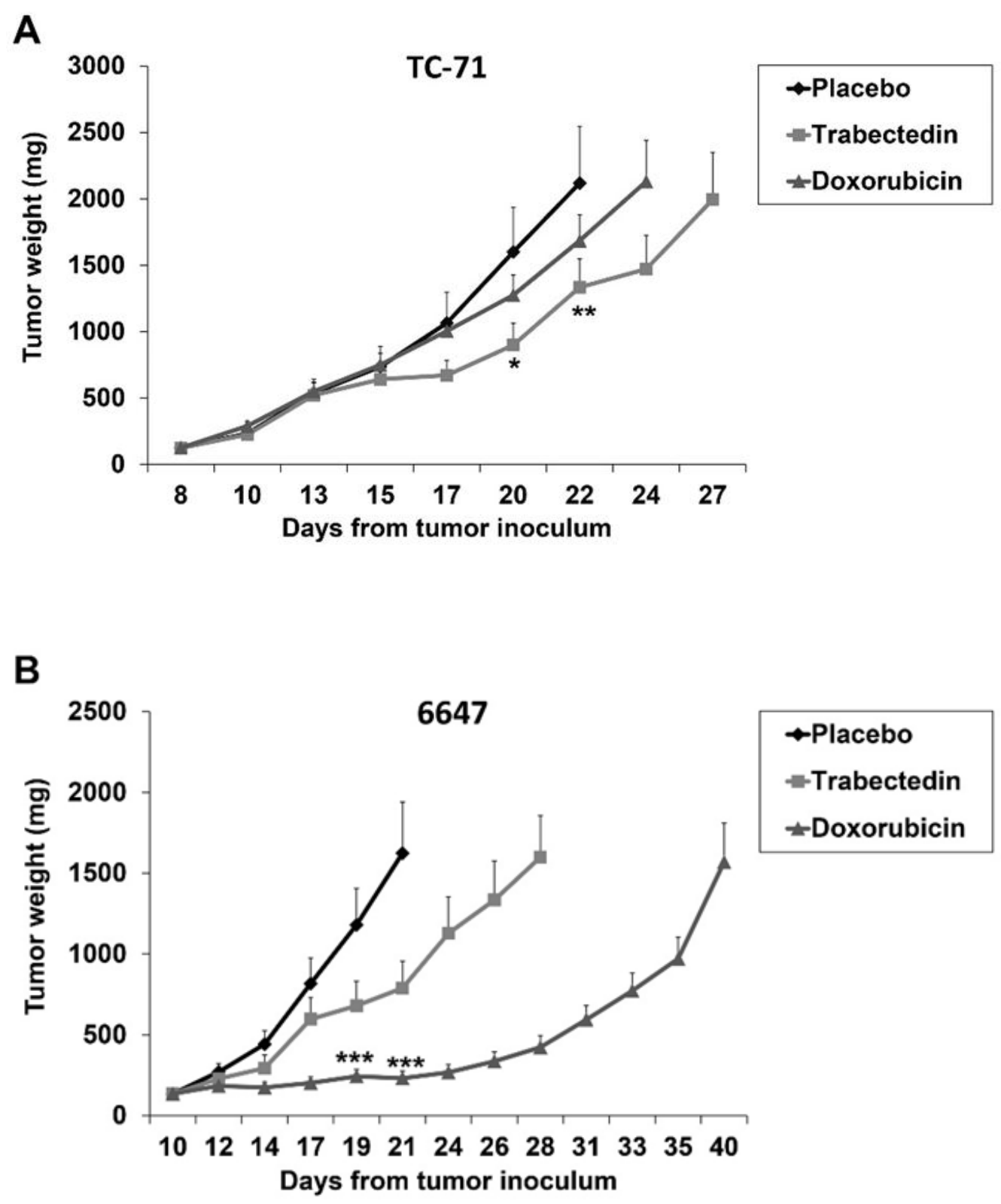

Figure 60S. Antitumor activity of Trabectedin and DXR in xenografts. (A) Antitumor activity of Trabectedin and DXR against TC71 ES xenograft (days of treatment 8,15 and 22). Anova test: ${ }^{*} p<0,05{ }^{* *} p<0,01$. (B) Antitumor activity of Trabectedin and DXR against 6647 ES xenograft. Anova test ${ }^{* *} p<0,001$. 
A

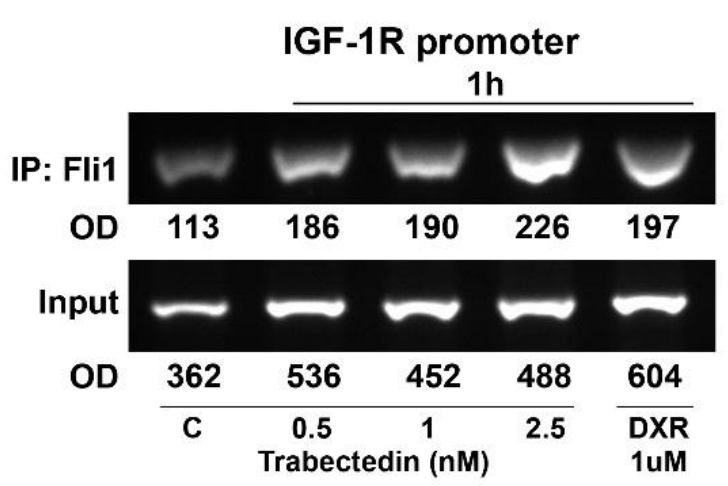

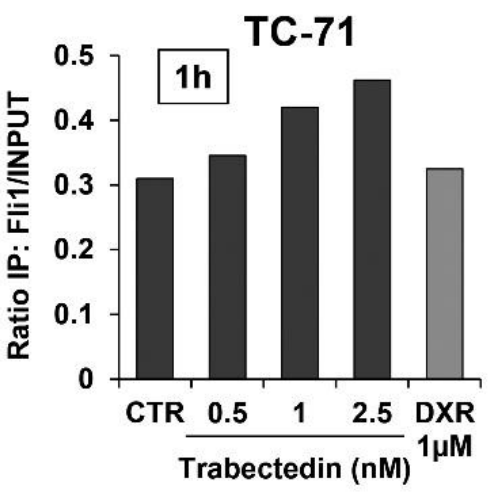

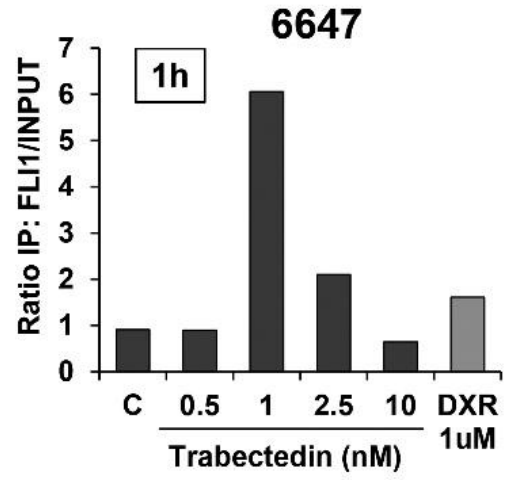

B
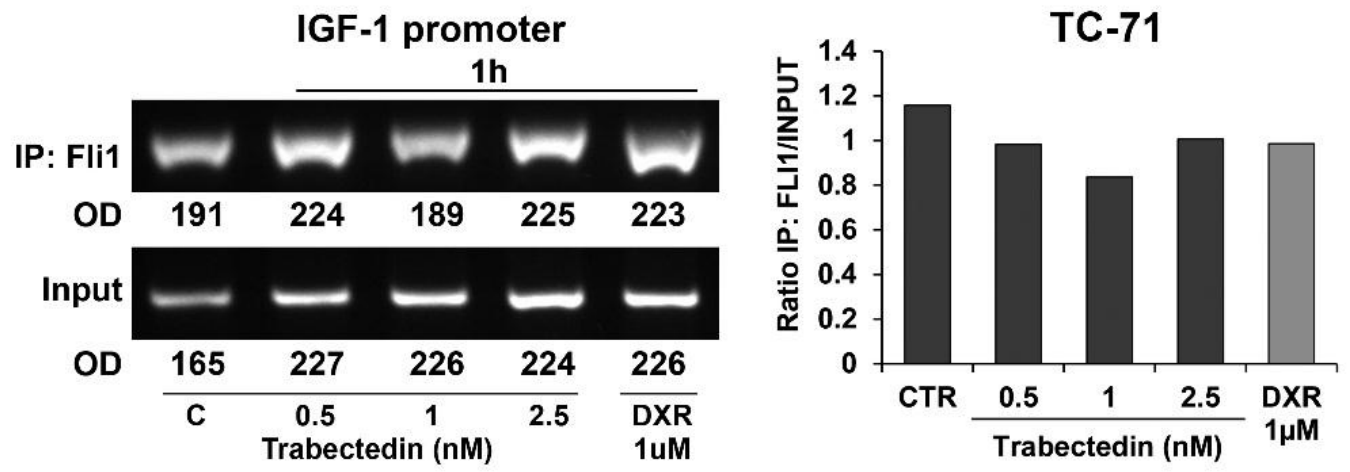

Figure 61S. Recruitment of IGFF1R promoter by EWS-Fli1 (A) Increased recruitment of EWS-Fli1 on IGF1R promoter in TC71 and 6647 ES cells treated 1h with Trabectedin. (B) EWS-FLI1 occupancy on the IGF1 promoter in TC71 cells treated for $1 \mathrm{~h}$ with Trabectedin and DXR. 


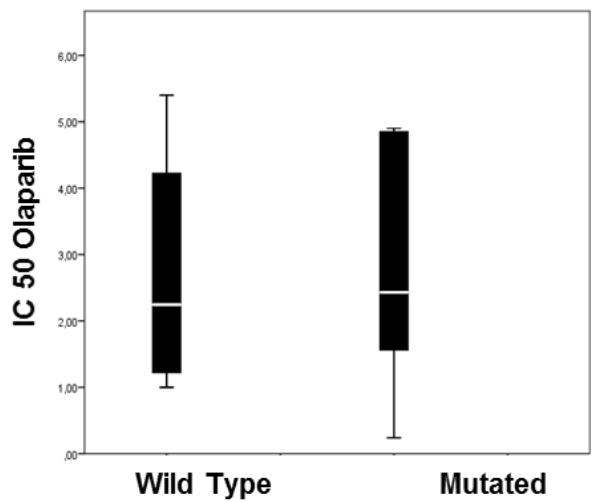

B

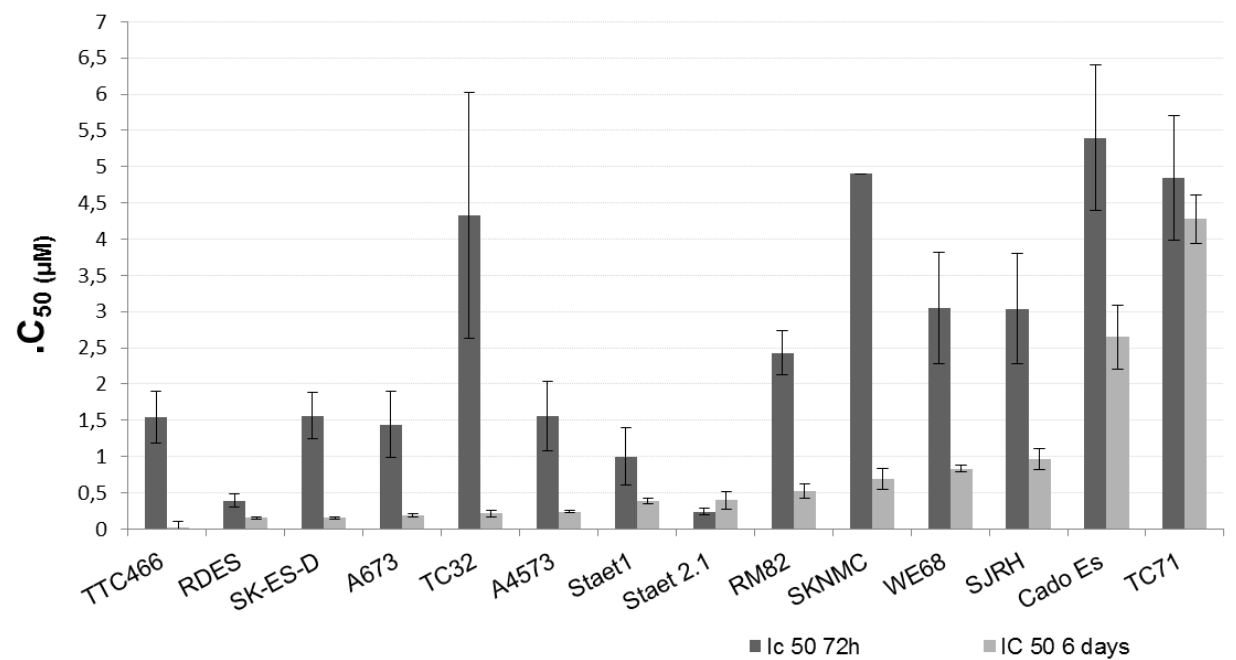

Figure 62S. Proliferation Inhibition after treatment with Olaparib. (A) Correlation between $\mathrm{IC}_{50}$ of proliferation to Olaparib and the status of p53. There was no correlation, $p>0,05$. (B) $I_{50}$ of proliferation after treatment with Olaparib: 72 hours versus 6 days.

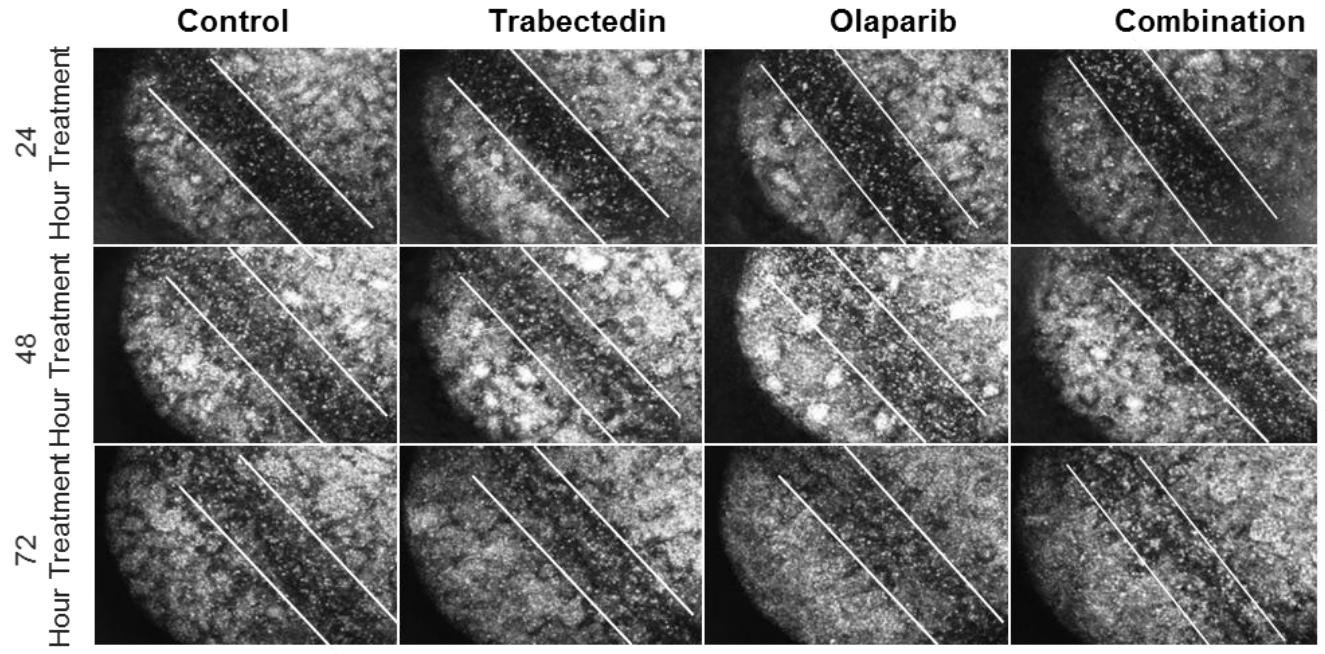

Figure 63S. Migration assays in RM82 cell line after treatment 
Table XXVIII.S. Fold Change from the LDMA after treatment with Trabectedin and/or Olaparib

$$
\text { Fold Change }
$$

(comparing to control group)

\begin{tabular}{|c|c|c|c|c|}
\hline Symbol & Unigene & Yondelis & Olaparib & Combination \\
\hline APEX1 & Hs.73722 & 0.64 & 1.018 & 0.8672 \\
\hline APEX2 & Hs.659558 & 0.7434 & 1.7092 & 1.8451 \\
\hline ATM & Hs.367437 & 0.8223 & 1.1876 & 1.069 \\
\hline ATR & Hs.271791 & 0.9984 & 1.3414 & 1.3968 \\
\hline ATXN3 & Hs.532632 & 0.7057 & 0.9577 & 0.7729 \\
\hline BRCA1 & Hs.194143 & 1.6442 & 1.4913 & 1.8691 \\
\hline BRCA2 & Hs.34012 & 1.0234 & 1.399 & 1.449 \\
\hline BRIP1 & Hs.532799 & 1.3821 & 1.9045 & 2.3385 \\
\hline CCNH & Hs.292524 & 0.9008 & 1.1443 & 1.2098 \\
\hline CCNO & Hs.3041 & 0.6936 & 1.2828 & 1.4174 \\
\hline CDK7 & Hs.184298 & 0.3131 & 1.3844 & 1.6298 \\
\hline DDB1 & Hs.290758 & 0.3241 & 1.0567 & 1.1474 \\
\hline DDB2 & Hs.700338 & 0.7444 & 1.2694 & 1.4446 \\
\hline DMC1 & Hs.339396 & 0.5471 & 0.9577 & 1.4324 \\
\hline ERCC1 & Hs.435981 & 0.5789 & 1.1404 & 1.2456 \\
\hline ERCC2 & Hs.487294 & 0.859 & 1.0832 & 1.0561 \\
\hline ERCC3 & Hs.469872 & 0.9135 & 1.1449 & 1.1692 \\
\hline ERCC4 & Hs.567265 & 1.079 & 1.2199 & 1.2171 \\
\hline ERCC5 & Hs.258429 & 1.0688 & 1.138 & 1.3476 \\
\hline ERCC6 & Hs. 654449 & 0.7418 & 1.3566 & 1.1502 \\
\hline
\end{tabular}


The Pathogenesis of Ewing Sarcoma / Appendix

\begin{tabular}{|c|c|c|c|c|}
\hline ERCC8 & Hs.435237 & 0.703 & 1.0584 & 0.9856 \\
\hline EX01 & Hs.498248 & 0.6029 & 1.9273 & 2.4661 \\
\hline FEN1 & Hs.409065 & 0.2417 & 1.2877 & 1.7279 \\
\hline LIG1 & Hs. 1770 & 0.168 & 1.025 & 1.1166 \\
\hline LIG3 & Hs.100299 & 0.5081 & 1.2577 & 0.9574 \\
\hline LIG4 & Hs.166091 & 0.8789 & 1.4908 & 1.6593 \\
\hline MGMT & Hs.501522 & 0.568 & 1.0537 & 0.534 \\
\hline MLH1 & Hs.195364 & 1.0108 & 1.0415 & 0.9544 \\
\hline MLH3 & Hs.436650 & 1.0298 & 1.4832 & 1.2214 \\
\hline MMS19 & Hs.500721 & 0.8353 & 1.2265 & 1.2222 \\
\hline MPG & Hs.459596 & 0.8467 & 1.1912 & 1.0777 \\
\hline MRE11A & Hs.192649 & 0.8344 & 1.0833 & 1.236 \\
\hline MSH2 & Hs.597656 & 0.7699 & 1.2399 & 1.4563 \\
\hline MSH3 & Hs.280987 & 0.321 & 1.3193 & 0.9042 \\
\hline MSH4 & Hs.216639 & 0.3384 & 1.234 & 0.2978 \\
\hline MSH5 & Hs.647011 & 0.1137 & 1.4945 & 1.4797 \\
\hline MSH6 & Hs.445052 & 0.7723 & 1.0411 & 1.1494 \\
\hline MUTYH & Hs.271353 & 0.7336 & 1.1551 & 1.1608 \\
\hline NEIL1 & Hs.512732 & 0.816 & 0.9557 & 0.8762 \\
\hline NEIL2 & Hs.293818 & 0.6822 & 0.9184 & 0.8625 \\
\hline NEIL3 & Hs.405467 & 1.3464 & 1.118 & 1.2745 \\
\hline NTHL1 & Hs.66196 & 0.3407 & 0.964 & 0.875 \\
\hline OGG1 & Hs.380271 & 1.2294 & 1.209 & 1.0776 \\
\hline PARP1 & Hs.177766 & 0.5906 & 0.9834 & 0.9337 \\
\hline PARP2 & Hs.409412 & 0.711 & 1.1688 & 1.8986 \\
\hline
\end{tabular}


The Pathogenesis of Ewing Sarcoma / Appendix

\begin{tabular}{|c|c|c|c|c|}
\hline PARP3 & Hs.271742 & 0.4062 & 1.6248 & 1.4864 \\
\hline PMS1 & Hs.111749 & 0.1247 & 0.9975 & 0.9253 \\
\hline PMS2 & Hs.632637 & 0.0016 & 1.0741 & 1.0127 \\
\hline PNKP & Hs.78016 & 0.9887 & 1.3108 & 1.3773 \\
\hline POLB & Hs.654484 & 1.123 & 1.1587 & 1.3479 \\
\hline POLD3 & Hs.82502 & 1.3943 & 1.5969 & 2.1602 \\
\hline POLL & Hs.523230 & 0.2838 & 1.1424 & 1.3329 \\
\hline PRKDC & Hs.491682 & 1.1391 & 1.3254 & 1.1871 \\
\hline RAD18 & Hs.375684 & 1.3467 & 1.5502 & 1.8189 \\
\hline RAD21 & Hs.81848 & 0.7353 & 1.0263 & 0.9866 \\
\hline RAD23A & Hs.643267 & 0.4415 & 1.1259 & 1.3637 \\
\hline RAD23B & Hs.521640 & 0.4044 & 0.7679 & 0.7029 \\
\hline RAD50 & Hs.655835 & 0.5595 & 1.344 & 1.1334 \\
\hline RAD51 & Hs.631709 & 0.0922 & 1.0308 & 1.2539 \\
\hline RAD51B & Hs.172587 & 0.0225 & 1.6129 & 0.5286 \\
\hline RAD51C & Hs.412587 & 0.5584 & 1.1362 & 1.1026 \\
\hline RAD51D & Hs.631757 & 0.9553 & 1.1158 & 0.9528 \\
\hline RAD52 & Hs.709202 & 1.2049 & 1.2517 & 1.4908 \\
\hline RAD54L & Hs.642042 & 0.6544 & 1.8858 & 2.5478 \\
\hline RFC1 & Hs.507475 & 1.2467 & 1.7001 & 2.1594 \\
\hline RPA1 & Hs.461925 & 0.8569 & 1.0146 & 1.0292 \\
\hline RPA3 & Hs.487540 & 0.6118 & 1.025 & 1.1033 \\
\hline SLK & Hs.591922 & 0.4988 & 1.1578 & 1.2302 \\
\hline SMUG1 & Hs.632721 & 0.463 & 1.1386 & 0.8381 \\
\hline TDG & Hs.584809 & 0.8917 & 1.0934 & 1.1476 \\
\hline
\end{tabular}


The Pathogenesis of Ewing Sarcoma / Appendix

\begin{tabular}{|c|c|c|c|c|}
\hline TOP3A & Hs.592115 & 0.3991 & 1.9027 & 2.5013 \\
\hline TOP3B & Hs.436401 & 0.1172 & 1.3949 & 1.5978 \\
\hline TREX1 & Hs.707026 & 0.8717 & 1.4947 & 1.6449 \\
\hline UNG & Hs.191334 & 1.1605 & 1.1664 & 1.5278 \\
\hline XAB2 & Hs.9822 & 1.126 & 1.3267 & 1.6688 \\
\hline XPA & Hs.654364 & 0.2149 & 0.9954 & 1.1093 \\
\hline XPC & Hs. 475538 & 0.4052 & 0.9739 & 0.973 \\
\hline XRCC1 & Hs.98493 & 1.1662 & 1.6094 & 1.8095 \\
\hline XRCC2 & Hs.647093 & 0.9138 & 1.3856 & 1.2881 \\
\hline XRCC3 & Hs.592325 & 0.3498 & 1.0326 & 1.143 \\
\hline XRCC4 & Hs.567359 & 0.33 & 1.0401 & 0.4037 \\
\hline XRCC5 & Hs.388739 & 0.5948 & 1.2094 & 1.1488 \\
\hline XRCC6 & Hs.292493 & 0.2469 & 1.1474 & 1.085 \\
\hline XRCC6BP1 & Hs.61188 & 0.2786 & 1.2561 & 1.286 \\
\hline
\end{tabular}




\section{Bibliography}


The Pathogenesis of Ewing Sarcoma / Bibliography 
1. Hanahan, D. and R.A. Weinberg, The hallmarks of cancer. Cell, 2000. 100(1): p. 57-70.

2. Hanahan, D. and R.A. Weinberg, Hallmarks of cancer: the next generation. Cell, 2011. 144(5): p. 646-74.

3. Helman, L.J. and P. Meltzer, Mechanisms of sarcoma development. Nat Rev Cancer, 2003. 3(9): p. 685-94.

4. Taylor, B.S., et al., Advances in sarcoma genomics and new therapeutic targets. Nat Rev Cancer, 2011. 11(8): p. 541-57.

5. van Geel, A.N., et al., Surgical treatment of lung metastases: The European Organization for Research and Treatment of Cancer-Soft Tissue and Bone Sarcoma Group study of 255 patients. Cancer, 1996. 77(4): p. 675-82.

6. Lawlor, E.R., et al., Peripheral primitive neuroectodermal tumors in adults: documentation by molecular analysis. J Clin Oncol, 1998. 16(3): p. 1150-7.

7. Linabery, A.M. and J.A. Ross, Childhood and adolescent cancer survival in the US by race and ethnicity for the diagnostic period 1975-1999. Cancer, 2008. 113(9): p. 257596.

8. Ladenstein, R., et al., Primary disseminated multifocal Ewing sarcoma: results of the Euro-EWING 99 trial. J Clin Oncol, 2011. 28(20): p. 3284-91.

9. Ludwig, J.A., Ewing sarcoma: historical perspectives, current state-of-the-art, and opportunities for targeted therapy in the future. Curr Opin Oncol, 2008. 20(4): p. 412-8.

10. Mackintosh, C., et al., 1q gain and CDT2 overexpression underlie an aggressive and highly proliferative form of Ewing sarcoma. Oncogene, 2012. 31(10): p. 1287-98.

11. Le Deley, M.C., et al., Impact of EWS-ETS fusion type on disease progression in Ewing's sarcoma/peripheral primitive neuroectodermal tumor: prospective results from the cooperative Euro-E.W.I.N.G. 99 trial. J Clin Oncol, 2010. 28(12): p. 1982-8.

12. van Doorninck, J.A., et al., Current treatment protocols have eliminated the prognostic advantage of type 1 fusions in Ewing sarcoma: a report from the Children's Oncology Group. J Clin Oncol, 2010. 28(12): p. 1989-94.

13. Hogendoorn, P.C., et al., Bone sarcomas: ESMO Clinical Practice Guidelines for diagnosis, treatment and follow-up. Ann Oncol, 2010. 21 Suppl 5: p. v204-13.

14. Haeusler, J., et al., The value of local treatment in patients with primary, disseminated, multifocal Ewing sarcoma (PDMES). Cancer, 2010. 116(2): p. 443-50.

15. de Alava, E., et al., EWS-FLI1 fusion transcript structure is an independent determinant of prognosis in Ewing's sarcoma. J Clin Oncol, 1998. 16(4): p. 1248-55.

16. Tirode, F., et al., Mesenchymal stem cell features of Ewing tumors. Cancer Cell, 2007. 11(5): p. 421-9.

17. Herrero-Martin, D., et al., Stable interference of EWS-FLI1 in an Ewing sarcoma cell line impairs IGF-1/IGF-1R signalling and reveals TOPK as a new target. Br J Cancer, 2009. 101(1): p. 80-90.

18. von Levetzow, C., et al., Modeling initiation of Ewing sarcoma in human neural crest cells. PLoS One. 6(4): p. e19305.

19. Friedenstein, A.J., R.K. Chailakhyan, and U.V. Gerasimov, Bone marrow osteogenic stem cells: in vitro cultivation and transplantation in diffusion chambers. Cell Tissue Kinet, 1987. 20(3): p. 263-72.

20. Krampera, M., et al., Immunological characterization of multipotent mesenchymal stromal cells--The International Society for Cellular Therapy (ISCT) working proposal. Cytotherapy, 2013. 15(9): p. 1054-61.

21. Dominici, M., et al., Minimal criteria for defining multipotent mesenchymal stromal cells. The International Society for Cellular Therapy position statement. Cytotherapy, 2006. 8(4): p. 315-7. 
22. Prieur, A., et al., EWS/FLI-1 silencing and gene profiling of Ewing cells reveal downstream oncogenic pathways and a crucial role for repression of insulin-like growth factor binding protein 3. Mol Cell Biol, 2004. 24(16): p. 7275-83.

23. Riggi, N., et al., Development of Ewing's sarcoma from primary bone marrow-derived mesenchymal progenitor cells. Cancer Res, 2005. 65(24): p. 11459-68.

24. Rubio, R., et al., Deficiency in $p 53$ but not retinoblastoma induces the transformation of mesenchymal stem cells in vitro and initiates leiomyosarcoma in vivo. Cancer Res. 70(10): p. 4185-94.

25. Matushansky, I., et al., Derivation of sarcomas from mesenchymal stem cells via inactivation of the Wnt pathway. J Clin Invest, 2007. 117(11): p. 3248-57.

26. Rodriguez, R., et al., Fus-Chop Fusion Protein Expression Coupled to P53 Deficiency Induces Liposarcoma in Mouse but not Human Adipose-Derived Mesenchymal Stem/Stromal Cells. Stem Cells.

27. Naka, N., et al., Synovial sarcoma is a stem cell malignancy. Stem Cells. 28(7): p. 111931.

28. Mohseny, A.B., et al., Osteosarcoma originates from mesenchymal stem cells in consequence of aneuploidization and genomic loss of Cdkn2. J Pathol, 2009. 219(3): p. 294-305.

29. Li, N., et al., Genetically transforming human mesenchymal stem cells to sarcomas: changes in cellular phenotype and multilineage differentiation potential. Cancer, 2009. 115(20): p. 4795-806.

30. Kauer, M., et al., A molecular function map of Ewing's sarcoma. PLoS One, 2009. 4(4): p. e5415.

31. Suva, M.L., et al., Identification of cancer stem cells in Ewing's sarcoma. Cancer Res, 2009. 69(5): p. 1776-81.

32. Turc-Carel, C., et al., Chromosomes in Ewing's sarcoma. I. An evaluation of 85 cases of remarkable consistency of t(11;22)(q24;q12). Cancer Genet Cytogenet, 1988. 32(2): $p$. 229-38.

33. Delattre, O., et al., Gene fusion with an ETS DNA-binding domain caused by chromosome translocation in human tumours. Nature, 1992. 359(6391): p. 162-5.

34. Kovar, H., Downstream EWS/FLI1 - upstream Ewing's sarcoma. Genome Med, 2010. 2(1): p. 8.

35. Kovar, H., Context matters: the hen or egg problem in Ewing's sarcoma. Semin Cancer Biol, 2005. 15(3): p. 189-96.

36. Tirado, O.M., et al., Caveolin-1 (CAV1) is a target of EWS/FLI-1 and a key determinant of the oncogenic phenotype and tumorigenicity of Ewing's sarcoma cells. Cancer Res, 2006. 66(20): p. 9937-47.

37. Ordonez, J.L., et al., Advances in Ewing's sarcoma research: where are we now and what lies ahead? Cancer Res, 2009. 69(18): p. 7140-50.

38. Grohar, P.J., et al., Identification of an inhibitor of the EWS-FLI1 oncogenic transcription factor by high-throughput screening. J Natl Cancer Inst, 2011. 103(12): p. 962-78.

39. Barber-Rotenberg, J.S., et al., Single enantiomer of $Y K-4-279$ demonstrates specificity in targeting the oncogene EWS-FLI1. Oncotarget, 2012. 3(2): p. 172-82.

40. Erkizan, H.V., et al., A small molecule blocking oncogenic protein EWS-FLI1 interaction with RNA helicase A inhibits growth of Ewing's sarcoma. Nat Med, 2009. 15(7): p. 7506.

41. Erkizan, H.V., et al., Novel peptide binds EWS-FLI1 and reduces the oncogenic potential in Ewing tumors. Cell Cycle, 2011. 10(19): p. 3397-408.

42. Toretsky, J.A., et al., Oncoprotein EWS-FLI1 activity is enhanced by RNA helicase A. Cancer Res, 2006. 66(11): p. 5574-81. 
43. Stegmaier, K., et al., Signature-based small molecule screening identifies cytosine arabinoside as an EWS/FLI modulator in Ewing sarcoma. PLoS Med, 2007. 4(4): p. e122.

44. Houghton, P.J., et al., Evaluation of cytarabine against Ewing sarcoma xenografts by the pediatric preclinical testing program. Pediatr Blood Cancer, 2010. 55(6): p. 1224-6.

45. Forni, C., et al., Trabectedin (ET-743) promotes differentiation in myxoid liposarcoma tumors. Mol Cancer Ther, 2009. 8(2): p. 449-57.

46. Grosso, F., et al., Efficacy of trabectedin (ecteinascidin-743) in advanced pretreated myxoid liposarcomas: a retrospective study. Lancet Oncol, 2007. 8(7): p. 595-602.

47. Manara, M.C., et al., The molecular mechanisms responsible for resistance to ET-743 (Trabectidin; Yondelis) in the Ewing's sarcoma cell line, TC-71. Int J Oncol, 2005. 27(6): p. 1605-16.

48. Molinski, T.F., et al., Drug development from marine natural products. Nat Rev Drug Discov, 2009. 8(1): p. 69-85.

49. Grohar, P.J., et al., Ecteinascidin 743 Interferes with the Activity of EWS-FLI1 in Ewing Sarcoma Cells. Neoplasia, 2011. 13(2): p. 145-53.

50. Duan, Z., et al., ZNF93 increases resistance to ET-743 (Trabectedin; Yondelis) and PM00104 (Zalypsis) in human cancer cell lines. PLoS One, 2009. 4(9): p. e6967.

51. Duan, Z., et al., Diverse cross-resistance phenotype to ET-743 and PM00104 in multidrug resistant cell lines. Cancer Chemother Pharmacol, 2009. 63(6): p. 1121-9.

52. Germano, G., et al., Role of macrophage targeting in the antitumor activity of trabectedin. Cancer Cell, 2013. 23(2): p. 249-62.

53. Lau, L., et al., A phase I and pharmacokinetic study of ecteinascidin-743 (Yondelis) in children with refractory solid tumors. A Children's Oncology Group study. Clin Cancer Res, 2005. 11(2 Pt 1): p. 672-7.

54. Guirouilh-Barbat, J., S. Antony, and Y. Pommier, Zalypsis (PM00104) is a potent inducer of gamma-H2AX foci and reveals the importance of the $C$ ring of trabectedin for transcription-coupled repair inhibition. Mol Cancer Ther, 2009. 8(7): p. 2007-14.

55. Leal, J.F., et al., Molecular pharmacology and antitumor activity of Zalypsis in several human cancer cell lines. Biochem Pharmacol, 2009. 78(2): p. 162-70.

56. Leal, J.F., et al., PM01183, a new DNA minor groove covalent binder with potent in vitro and in vivo anti-tumour activity. Br J Pharmacol, 2010. 161(5): p. 1099-110.

57. Ocio, E.M., et al., Zalypsis: a novel marine-derived compound with potent antimyeloma activity that reveals high sensitivity of malignant plasma cells to DNA double-strand breaks. Blood, 2009. 113(16): p. 3781-91.

58. Colado, E., et al., Zalypsis has in vitro activity in acute myeloid blasts and leukemic progenitor cells through the induction of a DNA damage response. Haematologica, 2011. 96(5): p. 687-95.

59. Bernard, G., et al., Apoptosis of immature thymocytes mediated by E2/CD99. J Immunol, 1997. 158(6): p. 2543-50.

60. Bernard, G., et al., CD99 (E2) up-regulates alpha4beta1-dependent $T$ cell adhesion to inflamed vascular endothelium under flow conditions. Eur J Immunol, 2000. 30(10): p. 3061-5.

61. Schenkel, A.R., et al., CD99 plays a major role in the migration of monocytes through endothelial junctions. Nat Immunol, 2002. 3(2): p. 143-50.

62. Dworzak, M.N., et al., CD99 (MIC2) expression in paediatric B-lineage leukaemia/lymphoma reflects maturation-associated patterns of normal $B$ lymphopoiesis. Br J Haematol, 1999. 105(3): p. 690-5.

63. Dworzak, M.N., et al., CD99 expression in T-lineage ALL: implications for flow cytometric detection of minimal residual disease. Leukemia, 2004. 18(4): p. 703-8.

64. Fisher, C., Synovial sarcoma. Ann Diagn Pathol, 1998. 2(6): p. 401-21. 
65. Bernard, G., et al., The E2 molecule (CD99) specifically triggers homotypic aggregation of CD4+CD8+ thymocytes. J Immunol, 1995. 154(1): p. 26-32.

66. Cerisano, V., et al., Molecular mechanisms of CD99-induced caspase-independent cell death and cell-cell adhesion in Ewing's sarcoma cells: actin and zyxin as key intracellular mediators. Oncogene, 2004. 23(33): p. 5664-74.

67. Houghton, P.J., et al., Initial testing of a monoclonal antibody (IMC-A12) against IGF-1R by the Pediatric Preclinical Testing Program. Pediatr Blood Cancer, 2010. 54(7): p. 9216.

68. Manara, M.C., et al., CD99 acts as an oncosuppressor in osteosarcoma. Mol Biol Cell, 2006. 17(4): p. 1910-21.

69. Pettersen, R.D., et al., CD99 signals caspase-independent T cell death. J Immunol, 2001. 166(8): p. 4931-42.

70. Sciandra, M., et al., CD99 drives terminal differentiation of osteosarcoma cells by acting as a spatial regulator of ERK 1/2. J Bone Miner Res, 2014. 29(5): p. 1295-309.

71. Zucchini, C., et al., CD99 suppresses osteosarcoma cell migration through inhibition of ROCK2 activity. Oncogene, 2014. 33(15): p. 1912-21.

72. Scotlandi, K., et al., CD99 engagement: an effective therapeutic strategy for Ewing tumors. Cancer Res, 2000. 60(18): p. 5134-42.

73. Rocchi, A., et al., CD99 inhibits neural differentiation of human Ewing sarcoma cells and thereby contributes to oncogenesis. J Clin Invest, 2010. 120(3): p. 668-80.

74. Sohn, H.W., et al., Engagement of CD99 induces apoptosis through a calcineurinindependent pathway in Ewing's sarcoma cells. Am J Pathol, 1998. 153(6): p. 1937-45.

75. Franzetti, G.A., et al., MiR-30a-5p connects EWS-FLI1 and CD99, two major therapeutic targets in Ewing tumor. Oncogene, 2012.

76. Scotlandi, K., et al., Targeting CD99 in association with doxorubicin: an effective combined treatment for Ewing's sarcoma. Eur J Cancer, 2006. 42(1): p. 91-6.

77. Scotlandi, K. and P. Picci, Targeting insulin-like growth factor 1 receptor in sarcomas. Curr Opin Oncol, 2008. 20(4): p. 419-27.

78. Martins, A.S., et al., IGF1R signaling in Ewing sarcoma is shaped by clathrin-/caveolindependent endocytosis. PLoS One, 2011. 6(5): p. e19846.

79. Benini, S., et al., Contribution of MEK/MAPK and PI3-K signaling pathway to the malignant behavior of Ewing's sarcoma cells: therapeutic prospects. Int J Cancer, 2004. 108(3): p. 358-66.

80. Benini, S., et al., Insulin-like growth factor binding protein 3 as an anticancer molecule in Ewing's sarcoma. Int J Cancer, 2006. 119(5): p. 1039-46.

81. Silvany, R.E., et al., Interference with the constitutive activation of ERK1 and ERK2 impairs EWS/FLI-1-dependent transformation. Oncogene, 2000. 19(39): p. 4523-30.

82. Scotlandi, K., et al., Expression of insulin-like growth factor system components in Ewing's sarcoma and their association with survival. Eur J Cancer, 2011. 47(8): p. 125866.

83. Pollak, M., Insulin and insulin-like growth factor signalling in neoplasia. Nat Rev Cancer, 2008. 8(12): p. 915-28.

84. Pollak, M., The insulin and insulin-like growth factor receptor family in neoplasia: an update. Nat Rev Cancer, 2012. 12(3): p. 159-69.

85. Pollak, M.N., E.S. Schernhammer, and S.E. Hankinson, Insulin-like growth factors and neoplasia. Nat Rev Cancer, 2004. 4(7): p. 505-18.

86. Amaral, A.T., et al., Innovative therapies in ewing sarcoma. Adv Anat Pathol, 2014. 21(1): p. 49-67.

87. Olmos, D., et al., Targeting the Insulin-Like Growth Factor 1 Receptor in Ewing's Sarcoma: Reality and Expectations. Sarcoma, 2011. 2011: p. 402508. 
88. Garofalo, C., et al., Identification of common and distinctive mechanisms of resistance to different anti-IGF-IR agents in Ewing's sarcoma. Mol Endocrinol, 2012. 26(9): p. 1603-16.

89. Garofalo, C., et al., Efficacy of and resistance to anti-IGF-1R therapies in Ewing's sarcoma is dependent on insulin receptor signaling. Oncogene, 2011. 30(24): p. 273040.

90. Macaulay, V.M., et al., Phase I study of humanized monoclonal antibody AVE1642 directed against the type 1 insulin-like growth factor receptor (IGF-1R), administered in combination with anticancer therapies to patients with advanced solid tumors. Ann Oncol, 2013. 24(3): p. 784-91.

91. Juergens, H., et al., Preliminary efficacy of the anti-insulin-like growth factor type 1 receptor antibody figitumumab in patients with refractory Ewing sarcoma. J Clin Oncol, 2011. 29(34): p. 4534-40.

92. Shin, D.H., et al., Akt/mTOR counteract the antitumor activities of cixutumumab, an anti-insulin-like growth factor I receptor monoclonal antibody. Mol Cancer Ther, 2011. 10(12): p. 2437-48.

93. Kolb, E.A., et al., Combination testing (Stage 2) of the Anti-IGF-1 receptor antibody IMC-A12 with rapamycin by the pediatric preclinical testing program. Pediatr Blood Cancer, 2012. 58(5): p. 729-35.

94. Huang, H.J., et al., R1507, an anti-insulin-like growth factor-1 receptor (IGF-1R) antibody, and EWS/FLI-1 siRNA in Ewing's sarcoma: convergence at the IGF/IGFR/Akt axis. PLoS One, 2011. 6(10): p. e26060.

95. Pappo, A.S., et al., R1507, a monoclonal antibody to the insulin-like growth factor 1 receptor, in patients with recurrent or refractory Ewing sarcoma family of tumors: results of a phase II Sarcoma Alliance for Research through Collaboration study. J Clin Oncol, 2011. 29(34): p. 4541-7.

96. Martins, A.S., et al., Insulin-like growth factor I receptor pathway inhibition by ADW742, alone or in combination with imatinib, doxorubicin, or vincristine, is a novel therapeutic approach in Ewing tumor. Clin Cancer Res, 2006. 12(11 Pt 1): p. 3532-40.

97. Martins, A.S., et al., A pivotal role for heat shock protein 90 in Ewing sarcoma resistance to anti-insulin-like growth factor 1 receptor treatment: in vitro and in vivo study. Cancer Res, 2008. 68(15): p. 6260-70.

98. Mangerich, A. and A. Burkle, How to kill tumor cells with inhibitors of poly(ADPribosyl)ation. Int J Cancer, 2011. 128(2): p. 251-65.

99. Rouleau, M., et al., PARP inhibition: PARP1 and beyond. Nat Rev Cancer, 2010. 10(4): $\mathrm{p}$. 293-301.

100. He, J.X., C.H. Yang, and Z.H. Miao, Poly(ADP-ribose) polymerase inhibitors as promising cancer therapeutics. Acta Pharmacol Sin, 2010. 31(9): p. 1172-80.

101. Helleday, T., Homologous recombination in cancer development, treatment and development of drug resistance. Carcinogenesis, 2010. 31(6): p. 955-60.

102. Brenner, J.C., et al., PARP-1 inhibition as a targeted strategy to treat Ewing's sarcoma. Cancer Res, 2012. 72(7): p. 1608-13.

103. Garnett, M.J., et al., Systematic identification of genomic markers of drug sensitivity in cancer cells. Nature, 2012. 483(7391): p. 570-5.

104. Brenner, J.C., et al., Mechanistic rationale for inhibition of poly(ADP-ribose) polymerase in ETS gene fusion-positive prostate cancer. Cancer Cell, 2011. 19(5): p. 664-78.

105. Herrero, A.B., et al., Cross-talk between nucleotide excision and homologous recombination DNA repair pathways in the mechanism of action of antitumor trabectedin. Cancer Res, 2006. 66(16): p. 8155-62. 
106. Soares, D.G., et al., Replication and homologous recombination repair regulate DNA double-strand break formation by the antitumor alkylator ecteinascidin 743. Proc Natl Acad Sci U S A, 2007. 104(32): p. 13062-7.

107. Tavecchio, M., et al., Role of homologous recombination in trabectedin-induced DNA damage. Eur J Cancer, 2008. 44(4): p. 609-18.

108. Guirouilh-Barbat, J., C. Redon, and Y. Pommier, Transcription-coupled DNA doublestrand breaks are mediated via the nucleotide excision repair and the Mre11-Rad50Nbs1 complex. Mol Biol Cell, 2008. 19(9): p. 3969-81.

109. Soares, D.G., et al., Trabectedin and its C subunit modified analogue PM01183 attenuate nucleotide excision repair and show activity toward platinum-resistant cells. Mol Cancer Ther, 2011. 10(8): p. 1481-9.

110. Vidal, A., et al., Lurbinectedin (PM01183), a new DNA minor groove binder, inhibits growth of orthotopic primary graft of cisplatin-resistant epithelial ovarian cancer. Clin Cancer Res, 2012. 18(19): p. 5399-411.

111. Gratias, S., et al., Genomic gains on chromosome 1q in retinoblastoma: consequences on gene expression and association with clinical manifestation. Int J Cancer, 2005. 116(4): p. 555-63.

112. O'Neil, N.J., D.M. van Pel, and P. Hieter, Synthetic lethality and cancer: cohesin and PARP at the replication fork. Trends Genet, 2013.

113. Ottaviano, L., et al., Molecular characterization of commonly used cell lines for bone tumor research: a trans-European EuroBoNet effort. Genes Chromosomes Cancer. 49(1): p. 40-51.

114. Alviano, F., et al., Term Amniotic membrane is a high throughput source for multipotent Mesenchymal Stem Cells with the ability to differentiate into endothelial cells in vitro. BMC Dev Biol, 2007. 7: p. 11.

115. Campagnoli, C., et al., Identification of mesenchymal stem/progenitor cells in human first-trimester fetal blood, liver, and bone marrow. Blood, 2001. 98(8): p. 2396-402.

116. Castrechini, N.M., et al., Mesenchymal stem cells in human placental chorionic villi reside in a vascular Niche. Placenta, 2010. 31(3): p. 203-12.

117. Cavallo, C., et al., Comparison of alternative mesenchymal stem cell sources for cell banking and musculoskeletal advanced therapies. J Cell Biochem, 2011. 112(5): p. 1418-30.

118. Parolini, O., et al., Concise review: isolation and characterization of cells from human term placenta: outcome of the first international Workshop on Placenta Derived Stem Cells. Stem Cells, 2008. 26(2): p. 300-11.

119. Pierdomenico, L., et al., Multipotent mesenchymal stem cells with immunosuppressive activity can be easily isolated from dental pulp. Transplantation, 2005. 80(6): p. 836-42.

120. Landuzzi, L., et al., The metastatic ability of Ewing's sarcoma cells is modulated by stem cell factor and by its receptor c-kit. Am J Pathol, 2000. 157(6): p. 2123-31.

121. Scotlandi, K., et al., C-kit receptor expression in Ewing's sarcoma: lack of prognostic value but therapeutic targeting opportunities in appropriate conditions. J Clin Oncol, 2003. 21(10): p. 1952-60.

122. Peruzzi, F., et al., Anti-apoptotic signaling of the insulin-like growth factor-I receptor through mitochondrial translocation of c-Raf and Nedd4. J Biol Chem, 2001. 276(28): p. 25990-6.

123. Baserga, R., F. Peruzzi, and K. Reiss, The IGF-1 receptor in cancer biology. Int J Cancer, 2003. 107(6): p. 873-7.

124. Pommier, Y., et al., DNA sequence- and structure-selective alkylation of guanine N2 in the DNA minor groove by ecteinascidin 743, a potent antitumor compound from the Caribbean tunicate Ecteinascidia turbinata. Biochemistry, 1996. 35(41): p. 13303-9. 
125. Riggi, N. and I. Stamenkovic, The Biology of Ewing sarcoma. Cancer Lett, 2007. 254(1): p. 1-10.

126. Johnson, J.J., et al., Prenatal and postnatal myeloid cells demonstrate stepwise progression in the pathogenesis of MLL fusion gene leukemia. Blood, 2003. 101(8): p. 3229-35.

127. Ladenstein, R., et al., Primary disseminated multifocal Ewing sarcoma: results of the Euro-EWING 99 trial. J Clin Oncol, 2010. 28(20): p. 3284-91.

128. Baruchel, S., et al., A phase 2 trial of trabectedin in children with recurrent rhabdomyosarcoma, Ewing sarcoma and non-rhabdomyosarcoma soft tissue sarcomas: a report from the Children's Oncology Group. Eur J Cancer, 2012. 48(4): p. 579-85.

129. Romano, M., et al., Comparison of in vitro and in vivo biological effects of trabectedin, lurbinectedin (PM01183) and Zalypsis(R) (PM00104). Int J Cancer, 2013. 133(9): p. 2024-33.

130. Casado, J.A., et al., Relevance of the Fanconi anemia pathway in the response of human cells to trabectedin. Mol Cancer Ther, 2008. 7(5): p. 1309-18.

131. Lessnick, S.L. and M. Ladanyi, Molecular pathogenesis of Ewing sarcoma: new therapeutic and transcriptional targets. Annu Rev Pathol, 2012. 7: p. 145-59.

132. Sankar, S., et al., Mechanism and relevance of EWS/FLI-mediated transcriptional repression in Ewing sarcoma. Oncogene, 2013. 32(42): p. 5089-100.

133. Grohar, P.J., et al., Dual targeting of EWS-FLI1 activity and the associated DNA damage response with Trabectedin and SN38 synergistically inhibits Ewing sarcoma cell growth. Clin Cancer Res, 2013.

134. Chen, C., et al., Differential disruption of EWS-FLI1 binding by DNA-binding agents. PLoS One, 2013. 8(7): p. e69714.

135. Manara, M.C., et al., Designing novel therapies against sarcomas in the era of personalized medicine and economic crisis. Curr Pharm Des, 2013. 19(30): p. 5344-61.

136. Soria, J.C., et al., A dose finding, safety and pharmacokinetic study of AVE1642, an antiinsulin-like growth factor-1 receptor (IGF-1R/CD221) monoclonal antibody, administered as a single agent and in combination with docetaxel in patients with advanced solid tumours. Eur J Cancer, 2013. 49(8): p. 1799-807.

137. Olmos, D., et al., Safety, pharmacokinetics, and preliminary activity of the anti-IGF-1R antibody figitumumab (CP-751,871) in patients with sarcoma and Ewing's sarcoma: a phase 1 expansion cohort study. Lancet Oncol, 2010. 11(2): p. 129-35.

138. Leiphrakpam, P.D., et al., In vivo analysis of insulin-like growth factor type 1 receptor humanized monoclonal antibody MK-0646 and small molecule kinase inhibitor OSI-906 in colorectal cancer. Oncol Rep, 2014. 31(1): p. 87-94.

139. Zinn, R.L., et al., ERK phosphorylation is predictive of resistance to IGF-1R inhibition in small cell lung cancer. Mol Cancer Ther, 2013. 12(6): p. 1131-9.

140. Kuijjer, M.L., et al., IR/IGF1R signaling as potential target for treatment of high-grade osteosarcoma. BMC Cancer, 2013. 13: p. 245.

141. Fox, E.M., et al., A kinome-wide screen identifies the insulin/IGF-I receptor pathway as a mechanism of escape from hormone dependence in breast cancer. Cancer Res, 2011. 71(21): p. 6773-84.

142. Kuhn, D.J., et al., Targeting the insulin-like growth factor-1 receptor to overcome bortezomib resistance in preclinical models of multiple myeloma. Blood, 2012. 120(16): p. 3260-70.

143. D'Incalci, M. and C.M. Galmarini, A review of trabectedin (ET-743): a unique mechanism of action. Mol Cancer Ther, 2010. 9(8): p. 2157-63.

144. Clark, N., X. Wu, and C. Her, MutS Homologues hMSH4 and hMSH5: Genetic Variations, Functions, and Implications in Human Diseases. Curr Genomics, 2013. 14(2): p. 81-90. 
145. Chitnis, M.M., et al., IGF-1R inhibition enhances radiosensitivity and delays doublestrand break repair by both non-homologous end-joining and homologous recombination. Oncogene, 2013.

146. Prasad, S.C., et al., Enhanced poly(adenosine diphosphate ribose) polymerase activity and gene expression in Ewing's sarcoma cells. Cancer Res, 1990. 50(1): p. 38-43.

147. Bryant, H.E., et al., Specific killing of BRCA2-deficient tumours with inhibitors of poly(ADP-ribose) polymerase. Nature, 2005. 434(7035): p. 913-7.

148. Farmer, $H_{\text {., }}$ et al., Targeting the DNA repair defect in BRCA mutant cells as a therapeutic strategy. Nature, 2005. 434(7035): p. 917-21.

149. Lee, H.J., et al., Combining PARP-1 inhibition and radiation in ewing sarcoma results in lethal DNA damage. Mol Cancer Ther, 2013. 12(11): p. 2591-600.

150. Mateo, J., et al., Appraising iniparib, the PARP inhibitor that never was-what must we learn? Nat Rev Clin Oncol, 2013. 10(12): p. 688-96.

151. Burdach, S., Molecular precision chemotherapy: overcoming resistance to targeted therapies? Clin Cancer Res, 2014. 20(5): p. 1064-6.

152. Pardali, E., et al., Critical role of endoglin in tumor cell plasticity of Ewing sarcoma and melanoma. Oncogene, 2011. 30(3): p. 334-45. 